\title{
EFFECTS OF A CATTLE FEEDLOT ON GROUND-WATER QUALITY IN THE SOUTH PLATTE RIVER VALLEY NEAR GREELEY, COLORADO
}

\section{U.S. GEOLOGICAL SURVEY}

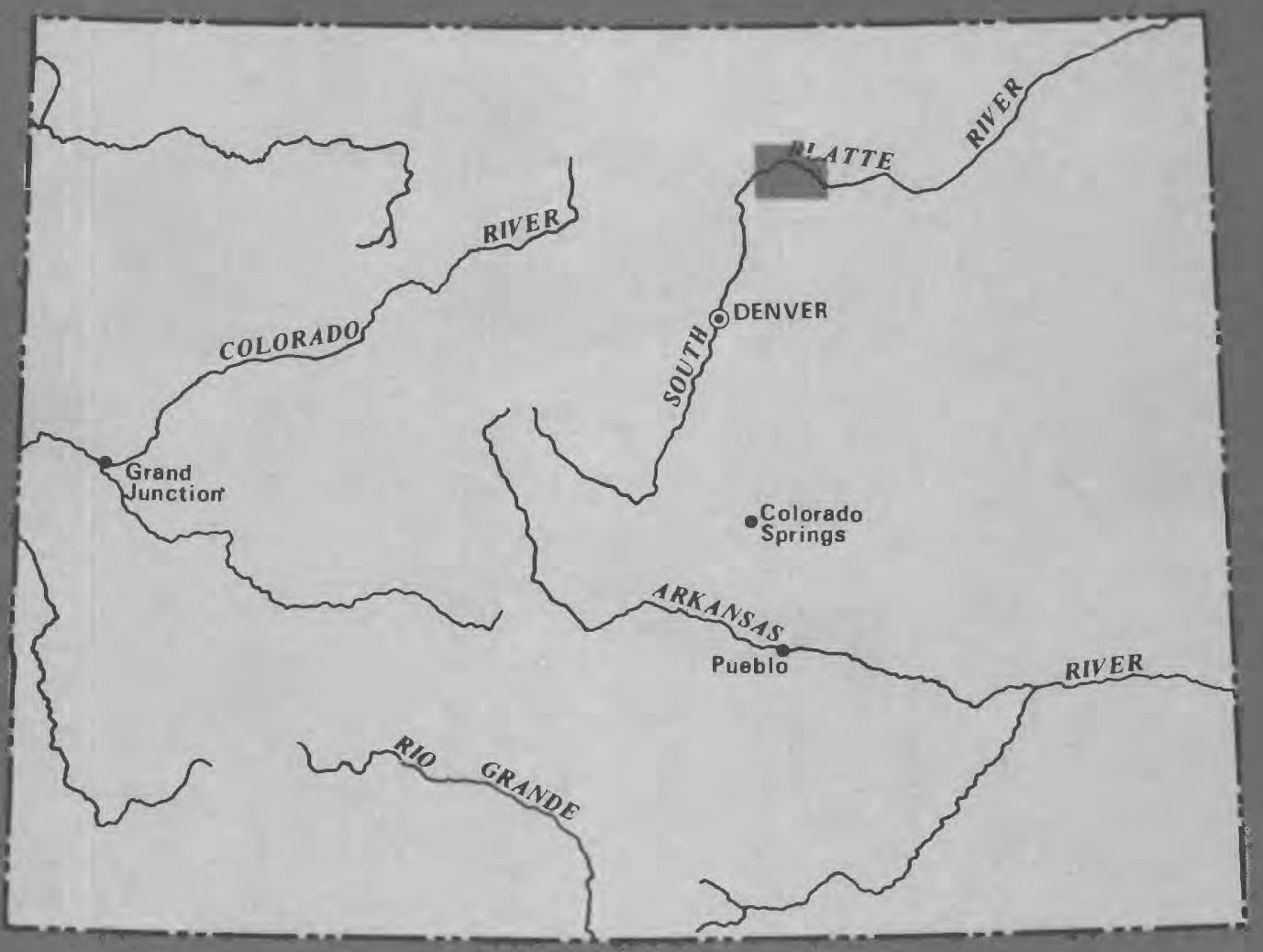

Water-Resources Investigations $80-83$

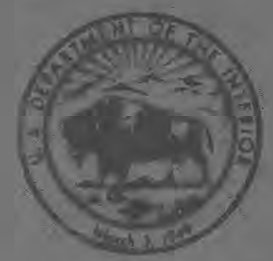




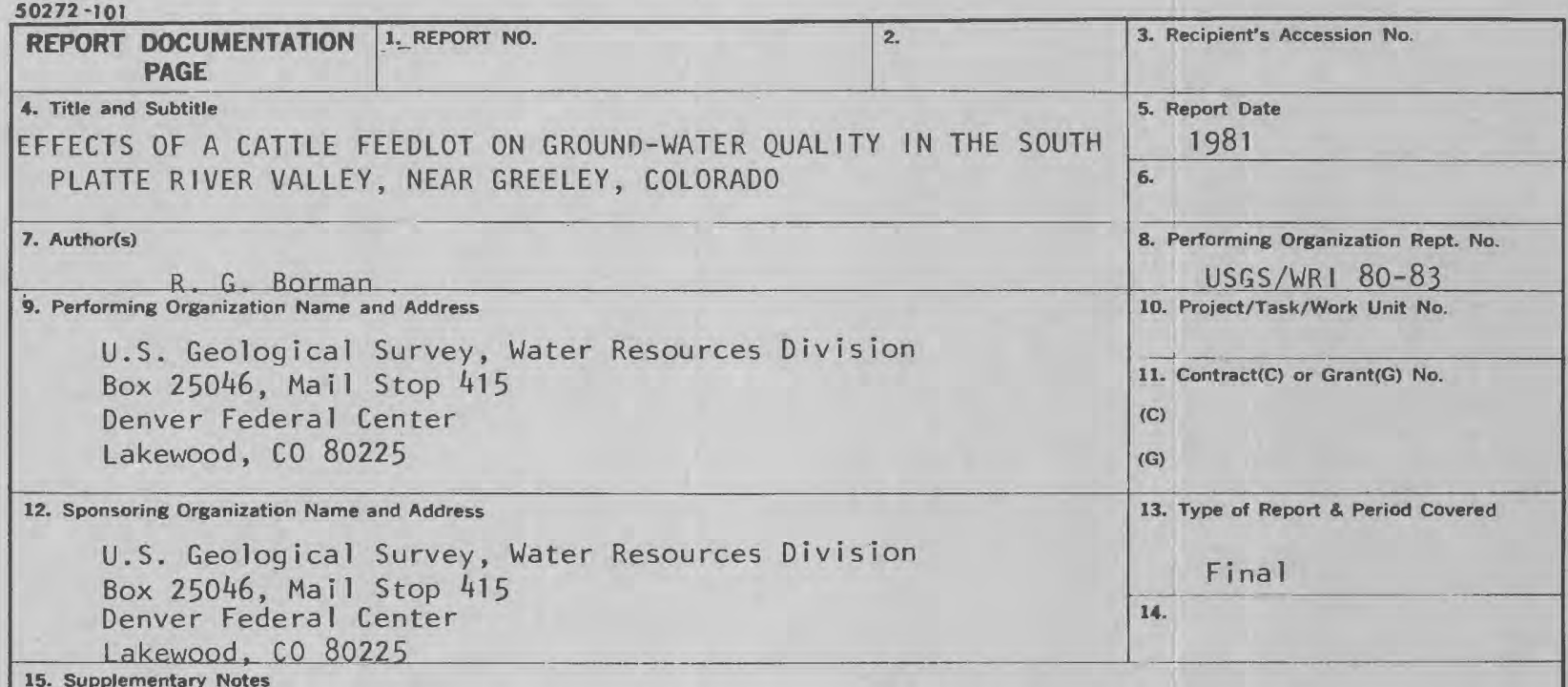

15. Supplementary Notes

\section{Abstract (Limit: 200 words)}

Ground-water quality may be changed by leachate from feedlots because large quantities of wastes are generated. The potential for water quality to be affected is especially high in alluvial aquifers with a shallow depth to water. However, monitoring water quality in 19 observation wells in and near a feedlot stocked with 90,000 head of beef cattle from April 1974, before the lot was stocked, to June 1978, has shown little change in ground-water quality that can be attributed to the feedlot. Analyses of water from two lysimeters in the unsaturated zone indicate leachate from the feedlot has percolated to a depth of at least 5 feet but not to a depth of 20 feet. The small changes in ground-water quality caused by the feedlot are $1 \mathrm{ikely}$ due to the $1 \mathrm{imited}$ available recharge, a relatively impermeable manure pack and soil clogging under the cattle pens resulting in slow vertical movement of leachate through the unsaturated zone, soil clogging under the unlined runoffretention ponds, and denitrification in the unsaturated zone.

17. Document Analysis a. Descriptors

Colorado, Ground water, Water quality, Nitrates, Farm wastes, Water table

b. Identifiers/Open-Ended Terms

Feedlot, South Platte River valley

c. COSATI Field/Group

18. Availabllity Statement

No restriction on distribution

\begin{tabular}{|l|l|}
\hline 19. Security Class (This Report) & $\begin{array}{l}\text { 21. No. of Pages } \\
82\end{array}$ \\
\hline 20. Security Class (This Page) & 22. Price \\
\hline
\end{tabular}


EFFECTS OF A CATTLE FEEDLOT ON GROUND-WATER

QUALITY IN THE SOUTH PLATTE RIVER VALLEY NEAR

GREELEY, COLORADO

By R. G. Borman

U.S. GEOLOGICAL SURVEY

Water-Resources Investigations $80-83$

Lakewood, Colorado

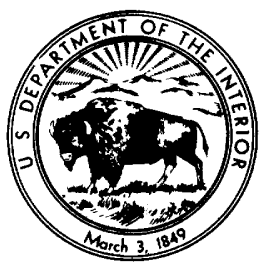




\section{UNITED STATES DEPARTMENT OF THE INTERIOR}

JAMES G. WATT, Secretary

GEOLOGICAL SURVEY

Doyle G. Frederick, Acting Director

For additional information write to:

Colorado District Chief

U.S. Geological Survey, MS 415

Box 25046, Denver Federal Center

Lakewood, co 80225 
Page

Abstract-1-0.- 1

Introduction-1.-

Ground-water hydrology-

Water qual ity- 12

Summary--

Selected references-- 20

Supplemental information- 23

ILLUSTRATIONS

Figures $1-3$. Maps showing:

Page

1. Location of study area-- 2

2. Location of wells, lysimeters, and runoff-retention ponds--...- 4

3. Bedrock topography-n 5

4. Geologic section through the feedlot-- 6

5-8. Maps showing:

5. Depth to bedrock- 7

6. Water table, March 1978- 9

7. Depth to water, March 1978- 10

8. Saturated thickness of the alluvial aquifer, March

1978-0.-.

9-13. Graphs showing:

9. Chloride concentrations in water from selected wells in and near the feedlot--.-.-.

10. Nitrate concentrations in water from selected wells in and near the feedlot--..- 15

11. Chloride concentrations in water from selected wells outside the feedlot-_... 16

12. Nitrate concentrations in water from selected wells outside the feedlot-- 16

13. Monthly precipitation at the feedlot and pan evaporation at Fort Collins-- 17

14. Diagram showing system of numbering wells using township,

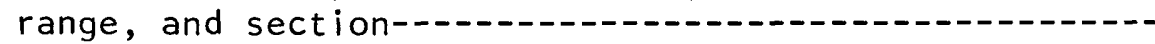


Table 1. Minimum, median, and maximum concentrations of selected properties and dissolved constituents in water from

19 observation wells, April 1974-

2. Comparison between median concentrations of selected chemical constituents in water from the alluvial aquifer and concentrations of selected chemical constituents in runoff from the feedlot-- 13

3. Chemical analyses of water from two lysimeters

4. Records of wells

5. Water-level records and chemical analyses of water from observation wells

6. Chemical analyses of water from domestic, stock, and irrigation wells 67

7. Chemical analyses of water from two runoff-retention ponds and from the feedlot water system supplied by eight wells-- 68

8. Logs of wells drilled by the U.S. Geological Survey-........ 70

9. Particle-size analyses, statistical characteristics, specific gravity, moisture content, total porosity, and vertical hydraulic conductivity for samples from observation wells

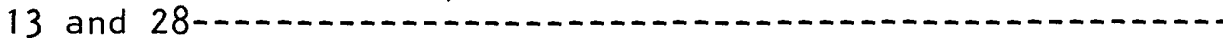

10. Monthly precipitation, in inches, from January 1975 to September 1978 at weather stations operated by the feedlot owner at the feedlot and by the U.S. Department of Commerce, National Weather Service at Greeley, Colo-.........

\section{CONVERSION FACTORS}

For the use of those readers who may prefer to use metric units rather than inch-pound units, the conversion factors for the terms used in this report are 1 isted below:

$\begin{array}{lc}\text { Multiply } & B y \\ \text { inch (in.) } & 25.40 \\ \text { foot (ft) } & 0.3048 \\ \text { mile (mi) } & 1.609 \\ \text { acre } & 0.4047 \\ \text { cubic foot }\left(\mathrm{ft}^{3}\right) & 0.02832 \\ \text { pound (lb) } & 0.04536 \\ \text { foot per day (ft/d) } & 0.3048 \\ \text { foot per mile } & 0.3048 \\ \text { foot per year (ft/yr) } & 0.3048 \\ \text { foot squared per day }\left(\mathrm{ft}^{2} / \mathrm{d}\right) & 0.0929\end{array}$

To obtain

millimeter

meter

ki lometer

hectare

cubic meter

ki logram

meter per day

meter per kilometer

meter per year

meter squared per day 


\title{
EFFECTS OF A CATTLE FEEDLOT ON GROUND-WATER QUALITY \\ IN THE SOUTH PLATTE RIVER VALLEY NEAR GREELEY, COLORADO
}

\author{
By R. G. Borman
}

\section{ABSTRACT}

Changes in water quality in an alluvial aquifer resulting from the operation of a feedlot stocked with 90,000 cattle have been minimal. Monitoring water quality in 19 observation wells from April 1974, prior to the operation of the feedlot, to June 1978, after about 4 years of operation, indicates that chloride concentrations have increased slightly in one well downgradient from a runoff-retention pond. Chemical analyses of water from two lysimeters installed in the unsaturated zone indicate that leachate from the feedlot has percolated to a depth of at least 5 feet but has not percolated to a depth of 20 feet. The small changes in ground-water quality caused by the feedlot are likely the result of the limited available recharge, a relatively impermeable manure pack, soil clogging under the cattle pens resulting in slow vertical movement of leachate through the unsaturated zone, soil clogging under the unlined runoff-retention ponds, and denitrification in the unsaturated zone.

\section{INTRODUCTION}

Large cattle feedlots may produce organic waste loads comparable to those produced by a medium-sized city. In the western United States, feedlots are often located in valleys where water is available for irrigating crops produced for livestock feed. These valleys frequently contain permeable alluvial deposits and have a shallow depth to ground water. The combination of large quantities of wastes, permeable deposits, and shallow depth to water increases the potential for changes in ground-water quality.

A site near Greeley, Colo. (fig. 1), about $50 \mathrm{mi}$ northeast of Denver, was chosen at which to monitor possible changes in ground-water quality resulting from operation of a feedlot. The climate in the area is semiarid, with an average annual precipitation of $11.47 \mathrm{in}$. during 1975-77, according to precipitation records collected at the feedlot by the operator. Stocking of the feedlot began April 26, 1974, and by the end of the year 50,000 cattle were fed each day at the site; by 1977, an average of 90,000 cattle were fed in the pens each day. 


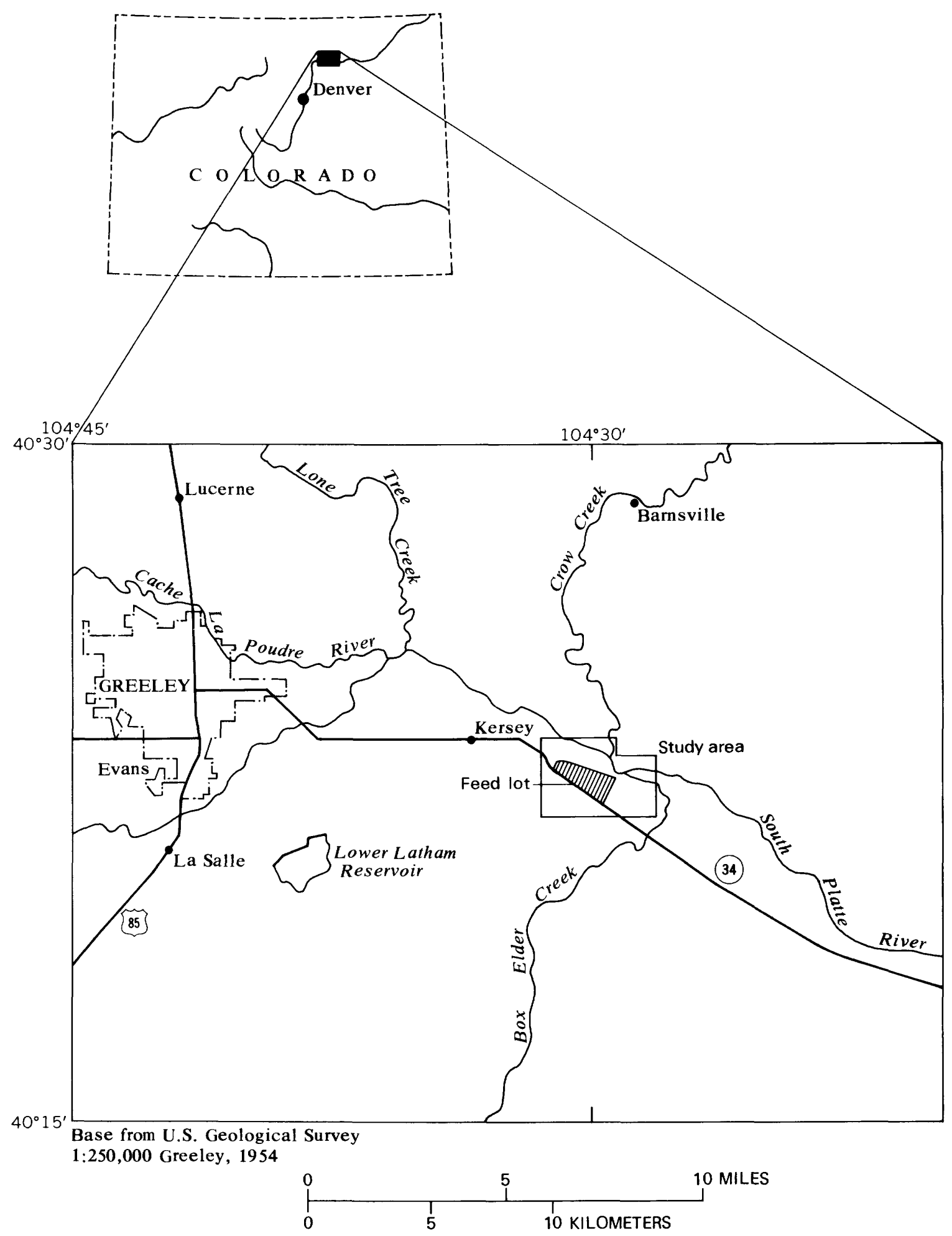

Figure 1-- Location of study area. 
The feedlot has an area of 500 acres of which 320 acres are fenced into pens. Storm runoff is collected in concrete-lined ditches and stored in two unlined retention ponds (fig. 2). The ponds also store overflow water from drinking troughs which are kept running during cold weather to prevent freezing. The storm water in the ponds is later mixed with water pumped from the aquifer and used for crop irrigation on adjacent land that is part of the feedlot complex. The pens do not have concrete or lined floors and are cleaned three or four times a year. The manure is used as fertilizer by landowners within $15 \mathrm{mi}$ of the feedlot.

Nineteen observation wells and four lysimeters, similar to those described by Wood (1973), were installed in and near the feedlot during construction (fig. 2). Water samples were collected from the wells for chemical analysis prior to stocking of the feedlot. After stocking, samples were collected quarterly from most wells until June 1978. Samples also were collected intermittently from two of the lysimeters, the runoff-retention ponds, and the water supply at the feedlot. Samples could not be successfully obtained from the other two lysimeters.

The purpose of this report is to present the data collected in the immediate vicinity of the feedlot and provide a preliminary interpretation of that data. The data-collection network was not designed to define the regional aspects of the geohydrologic system or the effects of waste removed from the immediate vicinity of the feedlot.

The cooperation of the feedlot owner for allowing the installation and monitoring of the wells and lysimeters and for providing data on precipitation, feedlot operations, and the feedlot supply wells is gratefully acknowledged.

\section{GROUND-WATER HYDROLOGY}

The feedlot is located on a terrace in the South Platte River valley about $30 \mathrm{ft}$ above the flood plain (fig. 2). The study area is underlain by alluvial sand and gravel deposits that comprise the aquifer.

The bedrock underlying the alluvial aquifer is the Laramie Formation of Late Cretaceous age. In the study area, the Laramie Formation primarily consists of shale that restricts vertical movement of water. Therefore, only the alluvial aquifer is subject to water-quality changes caused by the operation of the feedlot.

The altitude of the bedrock surface slopes from about 4,540 ft near the southwestern part of the study area to about $4,440 \mathrm{ft}$ in the northeastern part of the study area (figs. 3 and 4). The depth to bedrock ranges from about $40 \mathrm{ft}$ in the southwestern part of the study area to about $130 \mathrm{ft}$ in the north-central part of the study area (fig. 5). The depth to bedrock in the feedlot ranges from about $65 \mathrm{ft}$ near the southeastern runoff-retention pond to about $130 \mathrm{ft}$ in the northern part. 


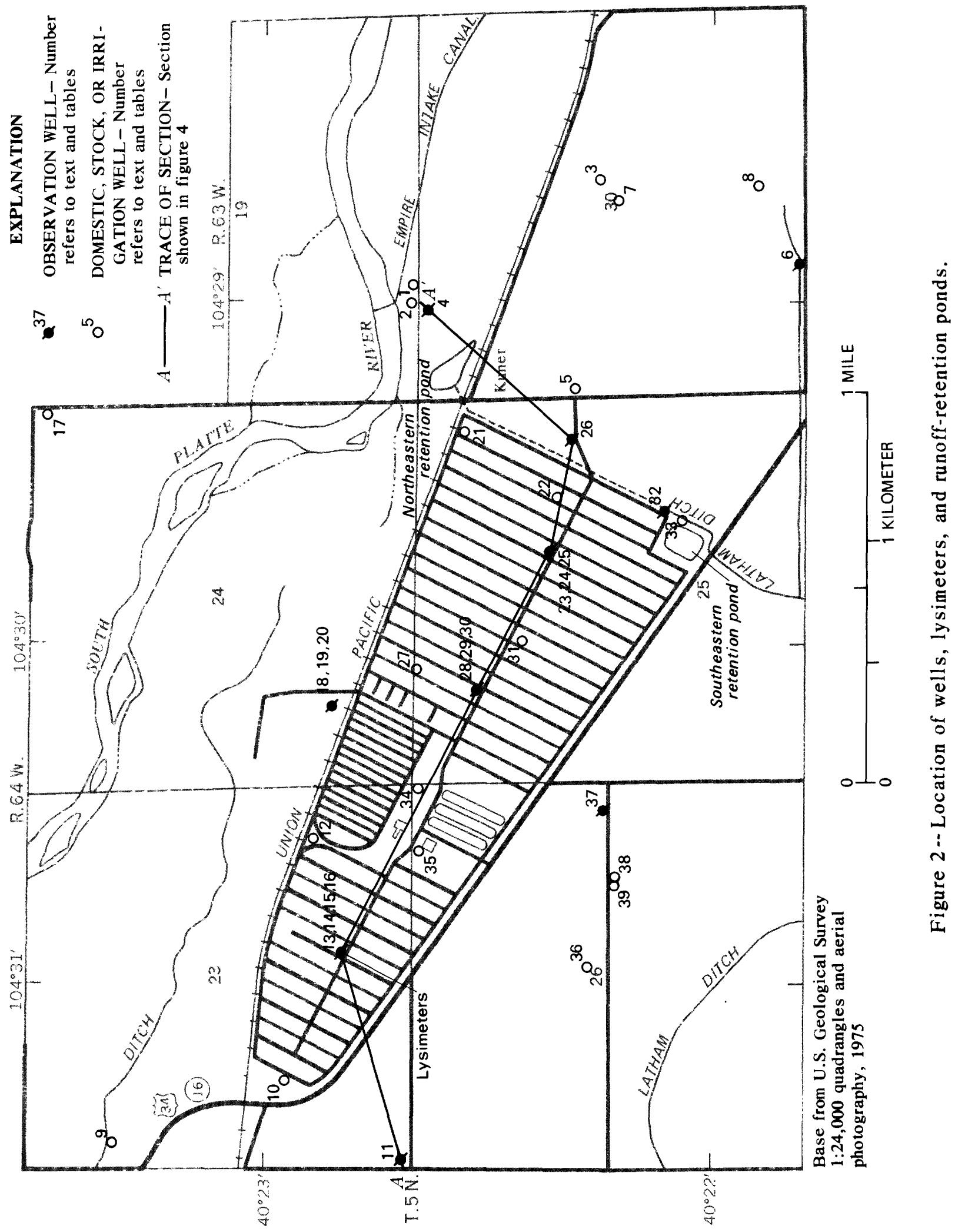




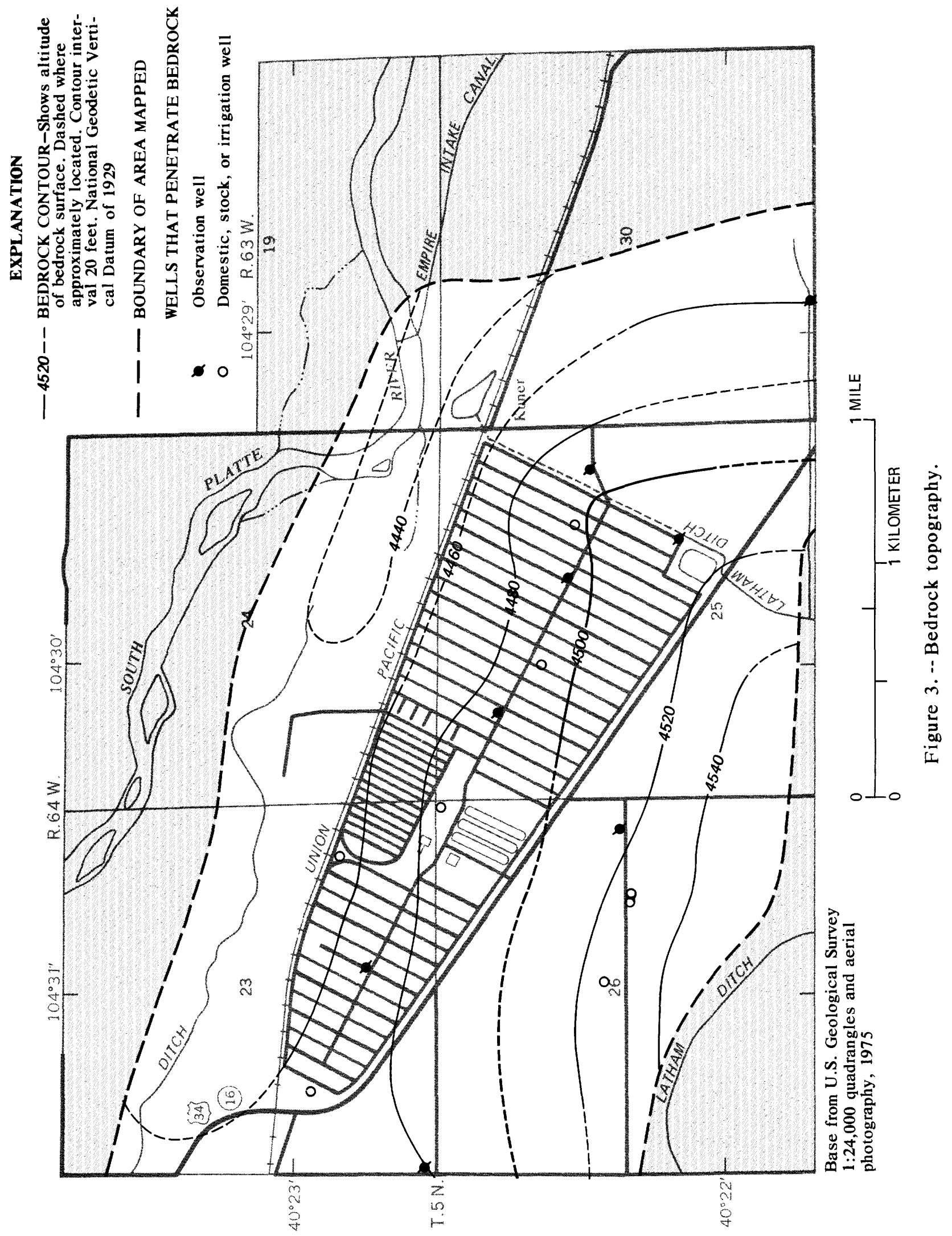




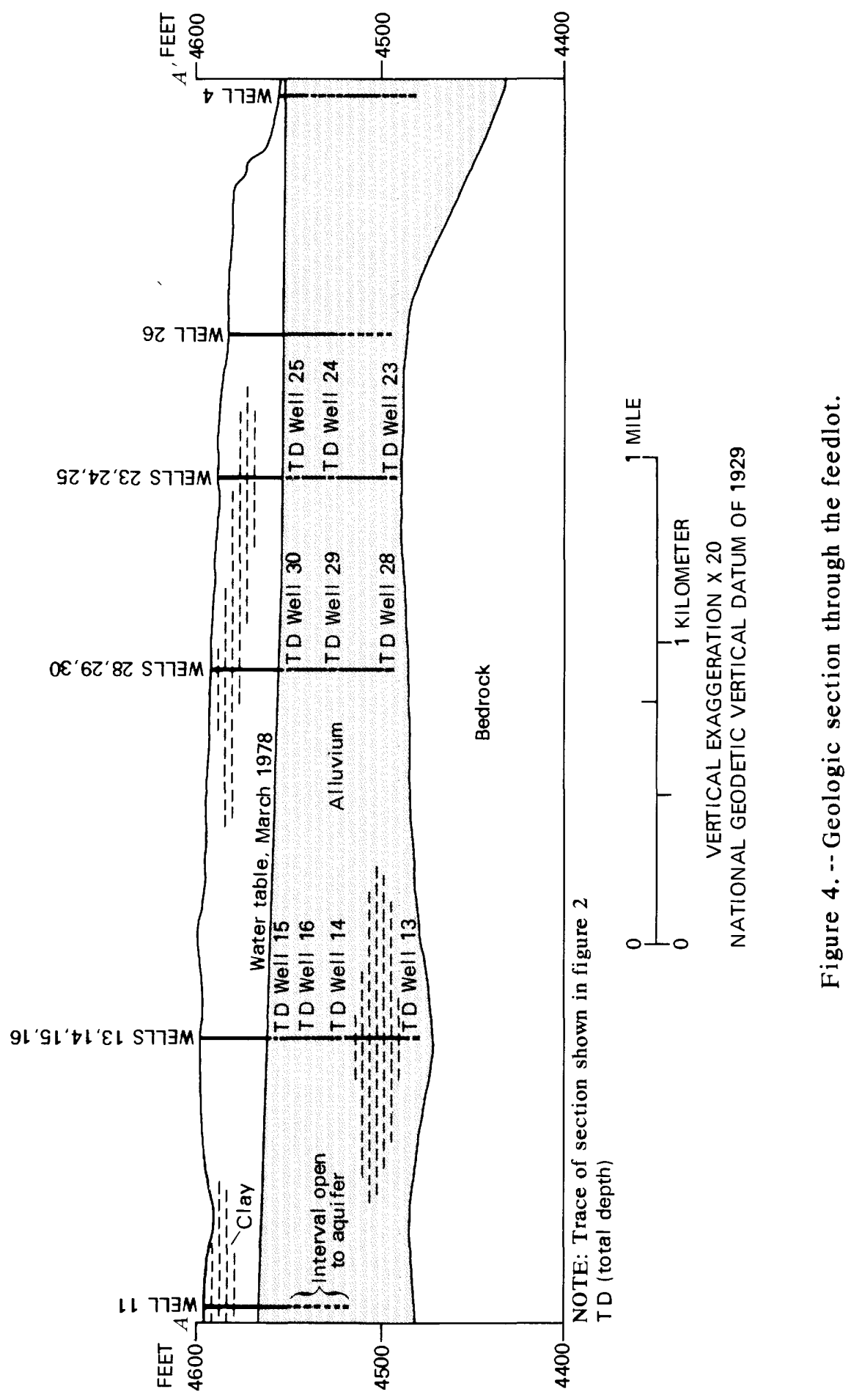




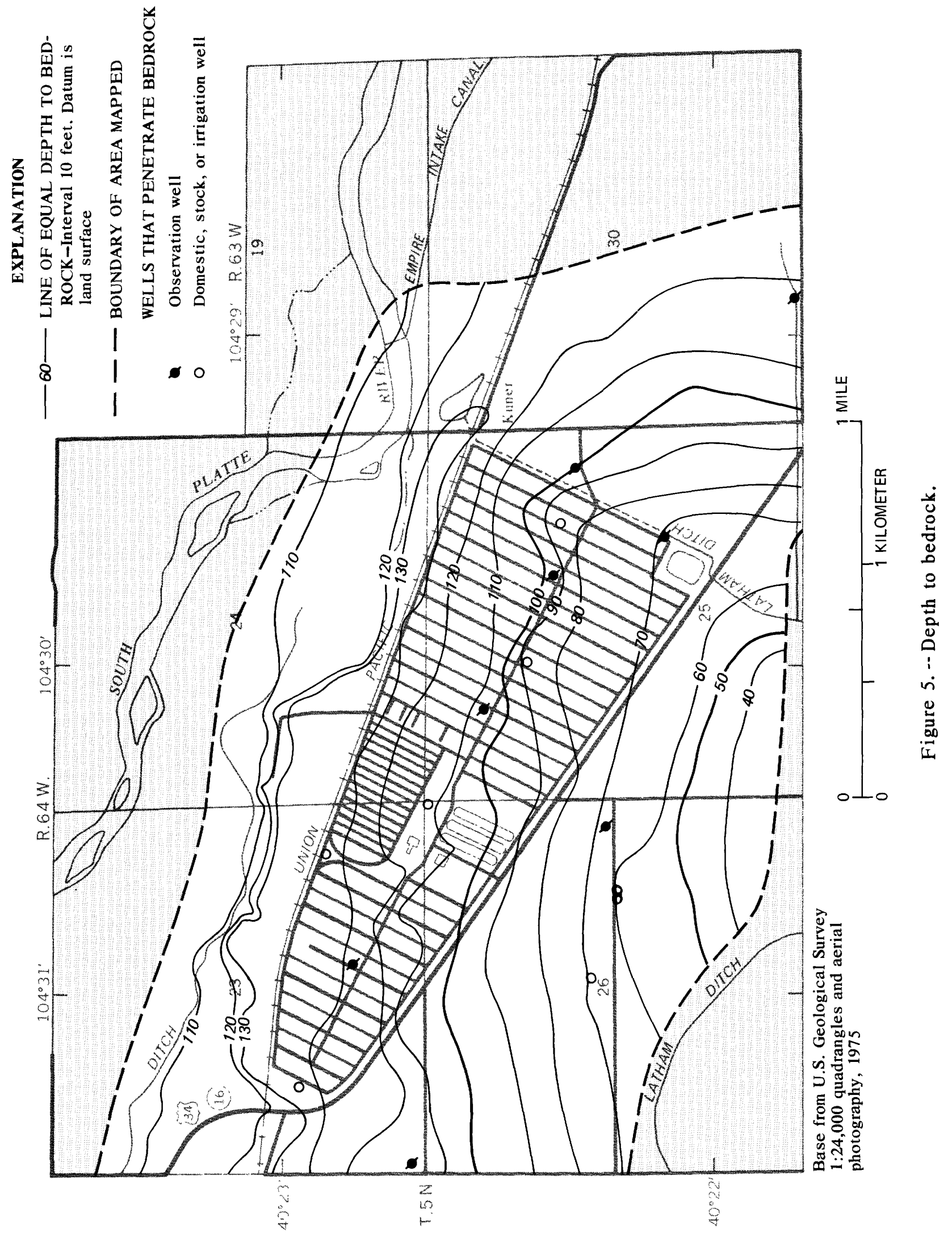


The aquifer underlying the feedlot is recharged by precipitation, the South Platte River, irrigation water applied to fields in excess of evapotranspiration, leakage from irrigation ditches and canals upgradient of the feedlot, and to some extent by infiltration of wastes from the livestock pens. Most of the water flowing beneath the feedlot entered the aquifer as recharge upgradient of the feedlot as indicated in figure 6 . However, the data used to construct figure 6 was collected at a time when the effects of precipitation, streamflow, evapotranspiration, and pumpage were minimal.

The altitude of the water table in the alluvial aquifer ranges from about 4,568 ft in the western part of the study area to about 4,550 $\mathrm{ft}$ in the eastern part of the study area (figs. 4 and 6 ). Water moves generally at right angles to the water-table contours from higher to lower altitudes and is moving approximately from west to east beneath the feedlot. The hydraulic gradient across the feedlot is about $8 \mathrm{ft} / \mathrm{mi}$. The depth to water in the study area ranges from zero at the South Platte River to about $35 \mathrm{ft}$ in the center of the feedlot (fig. 7).

The saturated thickness of the alluvial aquifer ranges from about $10 \mathrm{ft}$ in the southern part of the study area to about $110 \mathrm{ft}$ northeast of the feedlot (fig. 8). In the feedlot, the saturated thickness ranges from about $35 \mathrm{ft}$ near the southeastern runoff-retention pond to about $100 \mathrm{ft}$ along the northern edge of the feedlot.

Hydraulic conductivity of the aquifer beneath the feedlot is estimated to be $170 \mathrm{ft} / \mathrm{d}$, based on logs from 10 wells in the feedlot, and the porosity is estimated to be 0.4 on the basis of laboratory analyses of core samples from well 13 (depth interval 0 to $30 \mathrm{ft}$ ). The average ground-water velocity is estimated to be about $230 \mathrm{ft} / \mathrm{yr}$ based on the equation:

$$
V=\frac{K I}{\emptyset} \times 365
$$

where:

$$
\begin{aligned}
& V=\text { ground-water velocity, in feet per year, } \\
& K=\text { hydraulic conductivity, in feet per day, } \\
& I=\text { hydraulic gradient (dimensionless), and } \\
& \emptyset=\text { porosity (dimensionless). }
\end{aligned}
$$

This estimate of ground-water velocity is probably lower than the average rate of ground-water movement beneath the feedlot because (1) the laboratory value of porosity is probably about two times greater than the average effective porosity of the aquifer and (2) gradients greater than $8 \mathrm{ft} / \mathrm{mi}$ are produced by the pumping wells within the feedlot. 


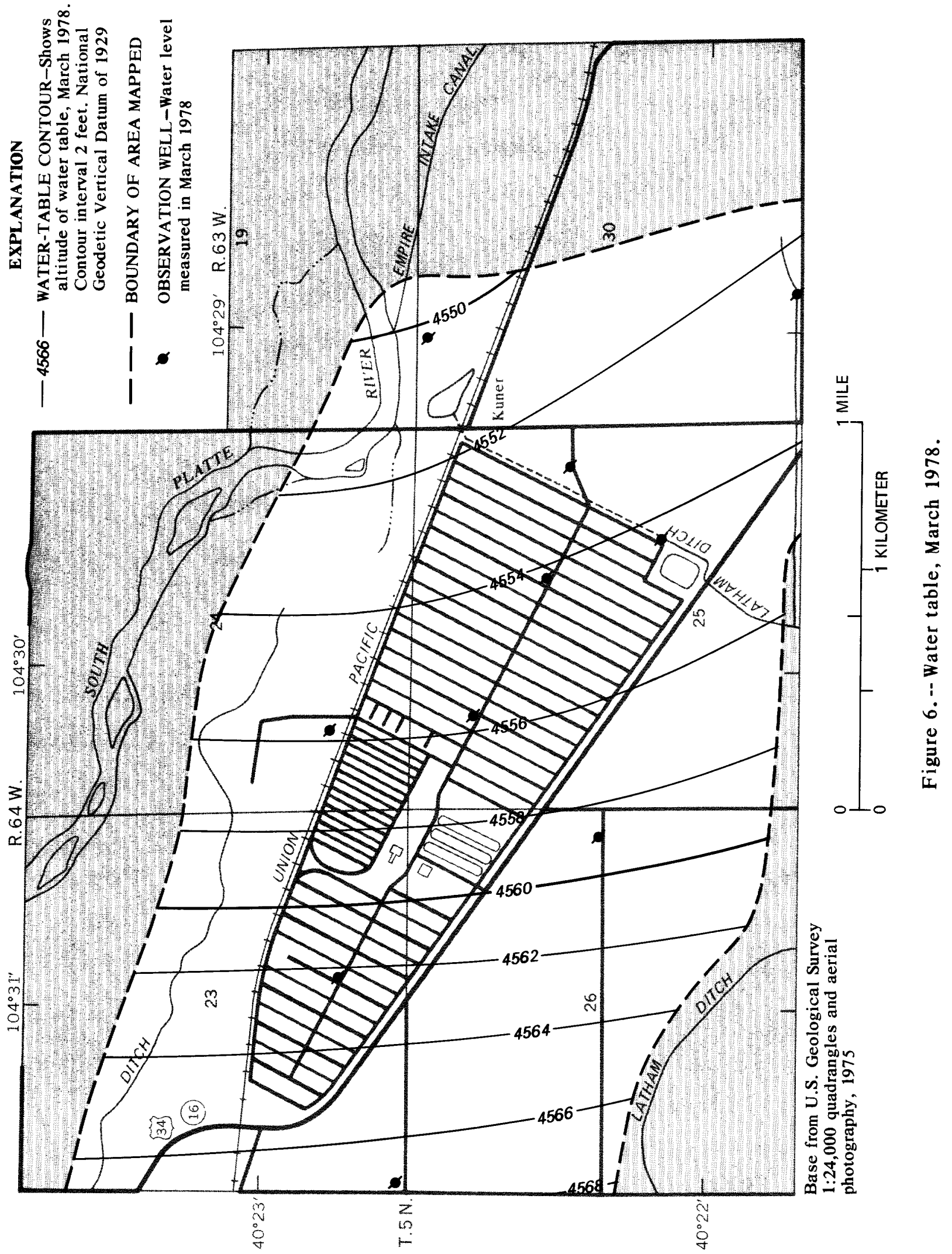




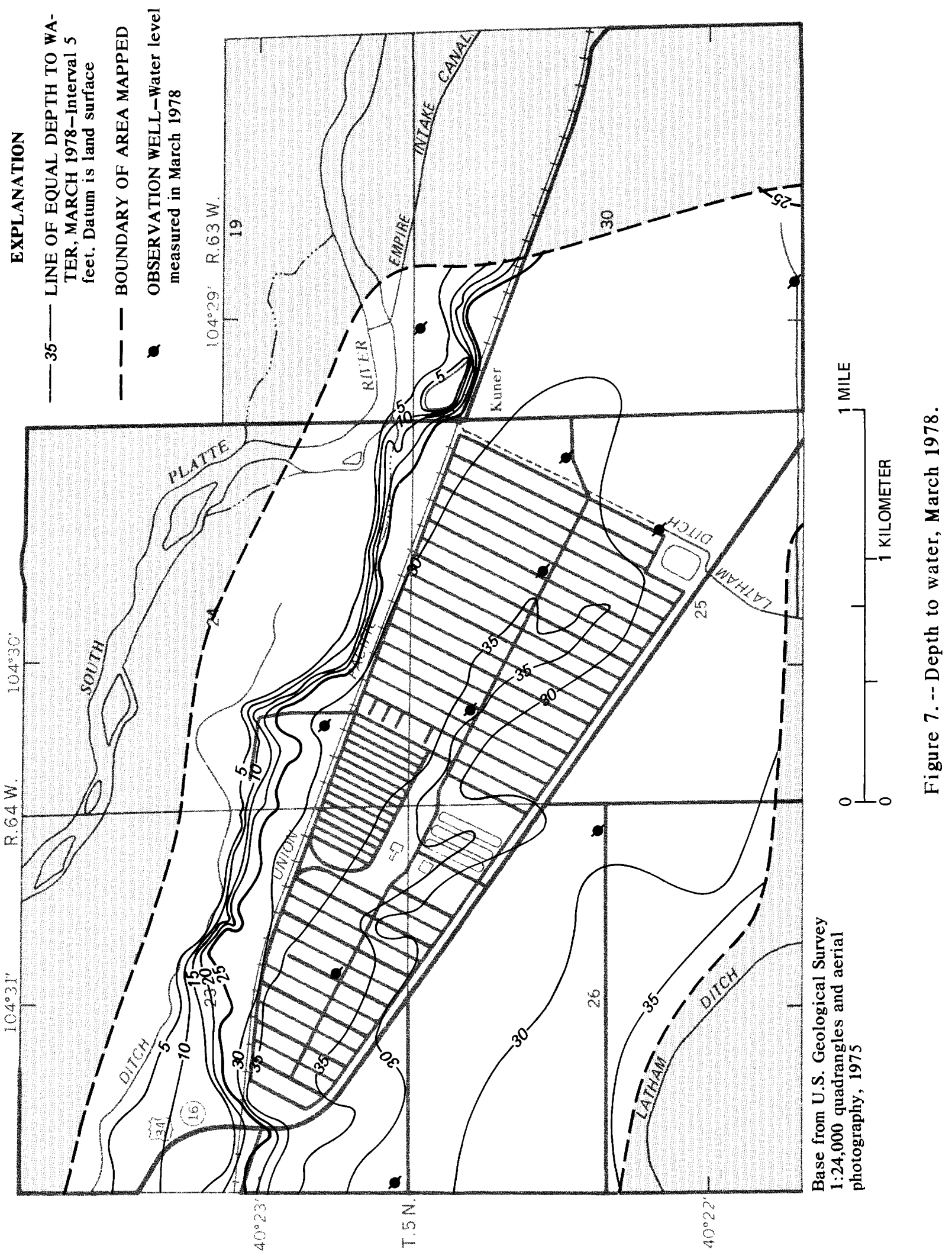




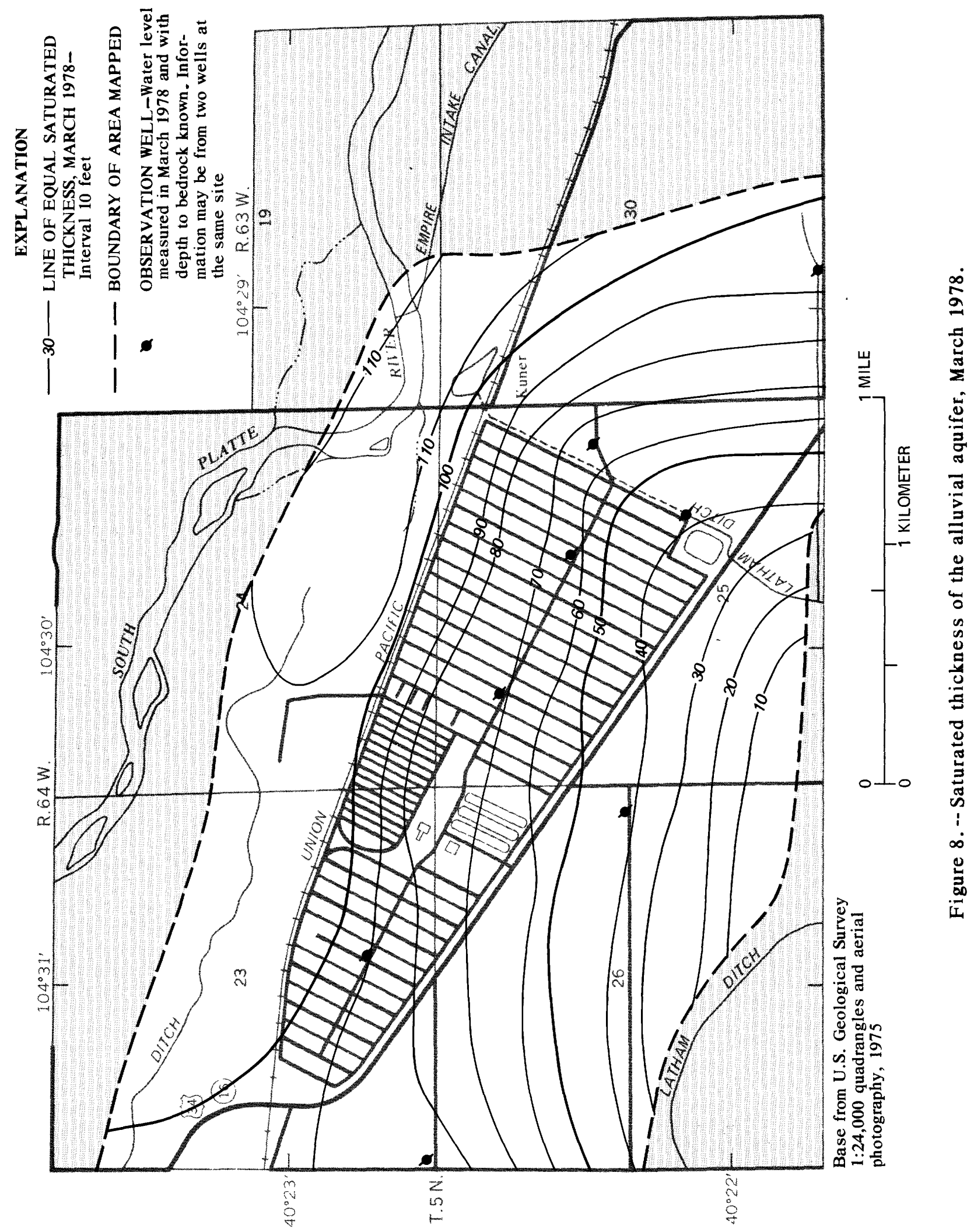


Chemical characteristics of water in the alluvial aquifer prior to stocking of the feedlot are summarized in table 1 . A comparison between selected dissolved mineral constituents in water in the aquifer prior to stocking of the feedlot and in runoff from the feedlot after stocking is shown in table 2. The sample of runoff was obtained from the southeastern runoff-retention pond following a storm in September 1976 when water quality should most closely represent that in leachate from the manure pack. (The chemical quality of water in the ponds varies as shown in table 7 in the Supplemental Information section at the back of this report, because overflow from drinking troughs as well as storm runoff is stored in the ponds.) Concentrations of all constituents used in the comparison except sulfate, sodium, and nitrate were greater in the runoff from the feedlot than the median content of water from the aquifer. Sodium concentration in the runoff may have been anomalous as McCalla, Ellis, Gilbertson, and Woods (1972) determined that the mean sodium concentration in feedlot wastes generally is about $1,050 \mathrm{mg} / \mathrm{L}$. The smaller concentration of nitrate in the runoff may have resulted because much of the nitrogen was present as ammonia and organic nitrogen and because denitrification was probably occurring.

Nitrate is the chemical constituent most likely to be increased in ground water by leachate from the feedlot because of the large quantities of nitrate in feedlot wastes. A steer produces wastes containing about $0.4 \mathrm{lb}$ of nitrogen daily (Taiganides and Hazen, 1966, p. 375). Therefore, the 90,000 cattle in the feedlot may produce about 36,000 lb of nitrogen daily. As much as 90 percent of the nitrogen in the manure may be lost to the atmosphere (McCalla and others, 1969, p. 5). If the remaining 10 percent of the nitrogen were mixed uniformly with the $106,000 \mathrm{ft}^{3}$ of ground water estimated to be moving beneath the feedlot daily, the concentration of nitrate as nitrogen in the water would be about $540 \mathrm{mg} / \mathrm{L}$.

Chloride is mobile in the ground-water environment and commonly is a good indicator of water-quality degradation (Robson, 1977, p. 13). Comparisons of nitrate and chloride concentrations from April 1974 to June 1978 and trends in the concentrations of these constituents were used to determine if the leachate from the feedlot had affected water quality in the aquifer.

There have been some changes in the quality of water from the 19 observation wells from April 1974, before the feedlot was stocked, to June 1978, but most changes appear to be unrelated to the feedlot. The median chloride concentration for samples from the 19 observation wells in June 1978 was $120 \mathrm{mg} / \mathrm{L}$, compared with $100 \mathrm{mg} / \mathrm{L}$ in April 1974. The median nitrate as nitrogen concentration was $7.7 \mathrm{mg} / \mathrm{L}$ in June 1978 , compared to $5.2 \mathrm{mg} / \mathrm{L}$ in April 1974. Increases in chloride and nitrate concentrations indicate some degradation of water quality. The variability of both chloride and nitrate concentrations is greater than observed changes and no major increasing trends for these constituents have been observed in water from wells most likely to be affected by leachate from the feedlot. 


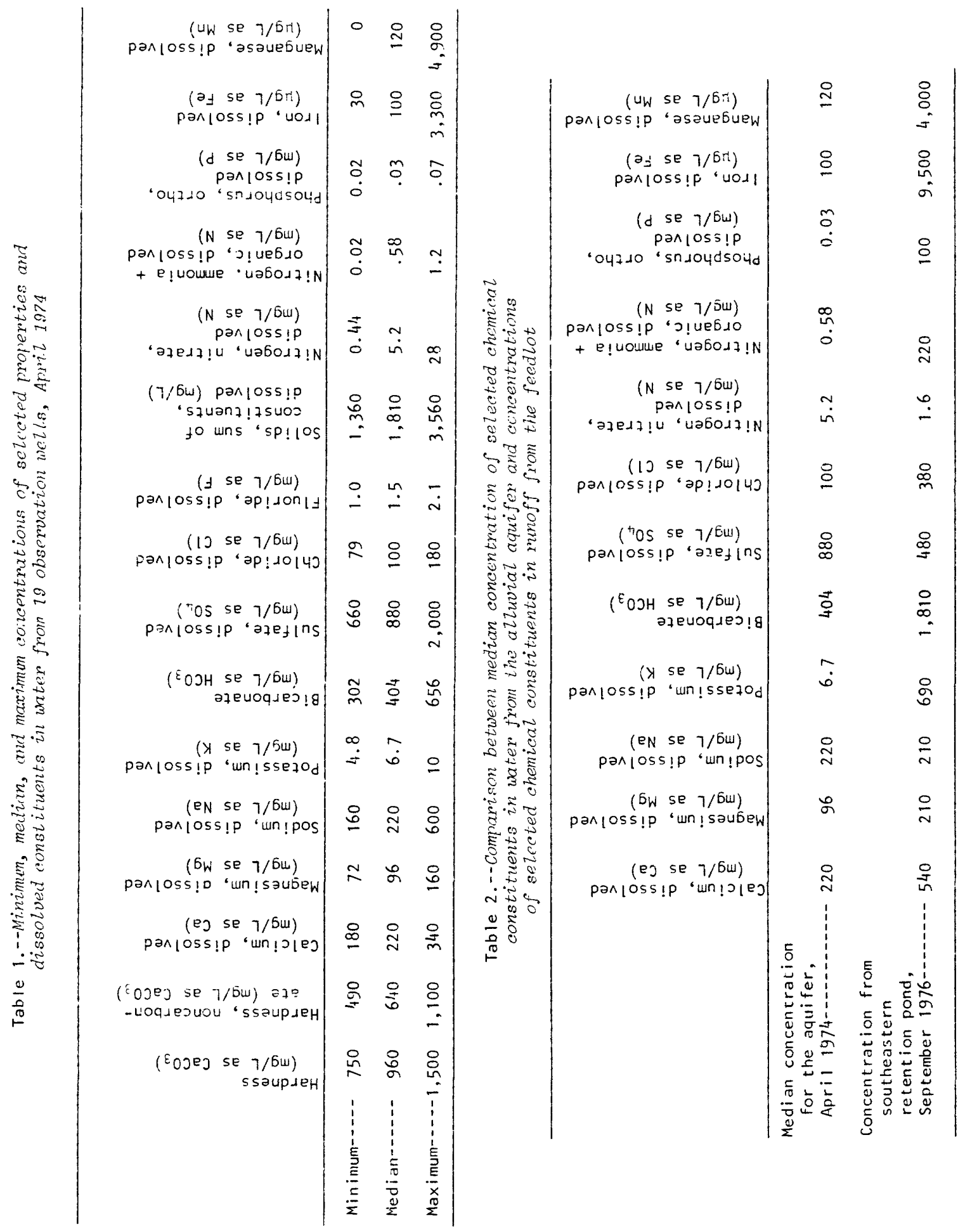


Chloride (fig. 9) and nitrate (fig. 10) concentrations in water from wells $16,25,26,30$, and 32 that are either in the feedlot or downgradient from the feedlot illustrate the lack of consistent trends. Neither chloride nor nitrate has increased in water from wells 16 and 30 . An overall but not steady increase in chloride concentrations and an overall but not steady decrease in nitrate concentrations has occurred in water from wells 25 and 26. If water in these wells was affected by leachate from the feedlot, an increase in both chloride and nitrate would be expected. A slightly more regular increase in chloride and a decrease in nitrate has occurred in water from well 32. This well is downgradient from the southeastern runoff-retention pond and water in the vicinity of the well may be slightly affected by runoff leaking from the pond. The decrease in the nitrate concentration in the well water may be due to the relatively small nitrate concentration in water in the runoff-retention ponds, which ranged from 0.04 to $3.7 \mathrm{mg} / \mathrm{L}$ as nitrogen in four analyses.

Water from wells 6, 11, and 37, which should not be affected by feedlot operations because they are not downgradient from the feedlot, showed some changes in chloride concentrations from April 1974 to June 1978 (fig. 11). Water from well 6 had a fairly constant chloride concentration with a slight increase in chloride concentration from April 1974 to June 1978. Water from wells 11 and 37 had an irregular trend in chloride concentrations with an overall slight increase in chloride concentrations from April 1974 to June 1978.

Nitrate concentrations in water from wells 6 and 11 have been fairly constant with little overall change during the monitoring period (fig. 12). Water from well 37 had some variations in nitrate concentrations and a slight increase in nitrate from April 1974 to March 1978. There was a large increase in nitrate concentrations from March to June 1978 in water from this well.

The changes in water quality from sample to sample from the same well are due to differences in chemical quality of water moving past the well. These differences in water quality in the aquifer with time are caused largely by differences in concentrations of chemical constituents in recharge water reaching the aquifer, mostly upgradient of the sampling point. In the South Platte River valley, much recharge comes from irrigation water, both applied to fields and leaking from irrigation canals and ditches. The chemical quality of this water changes from year to year and seasonally. The quantity of irrigation water also changes seasonally. Pulses of recharge water with differences in chemical quality reach the aquifer and then move downgradient, causing differences in chemical quality with time at any sampling point. 
Figure 4 and table 4 show well depths and the intervals of the wells open to the aquifer

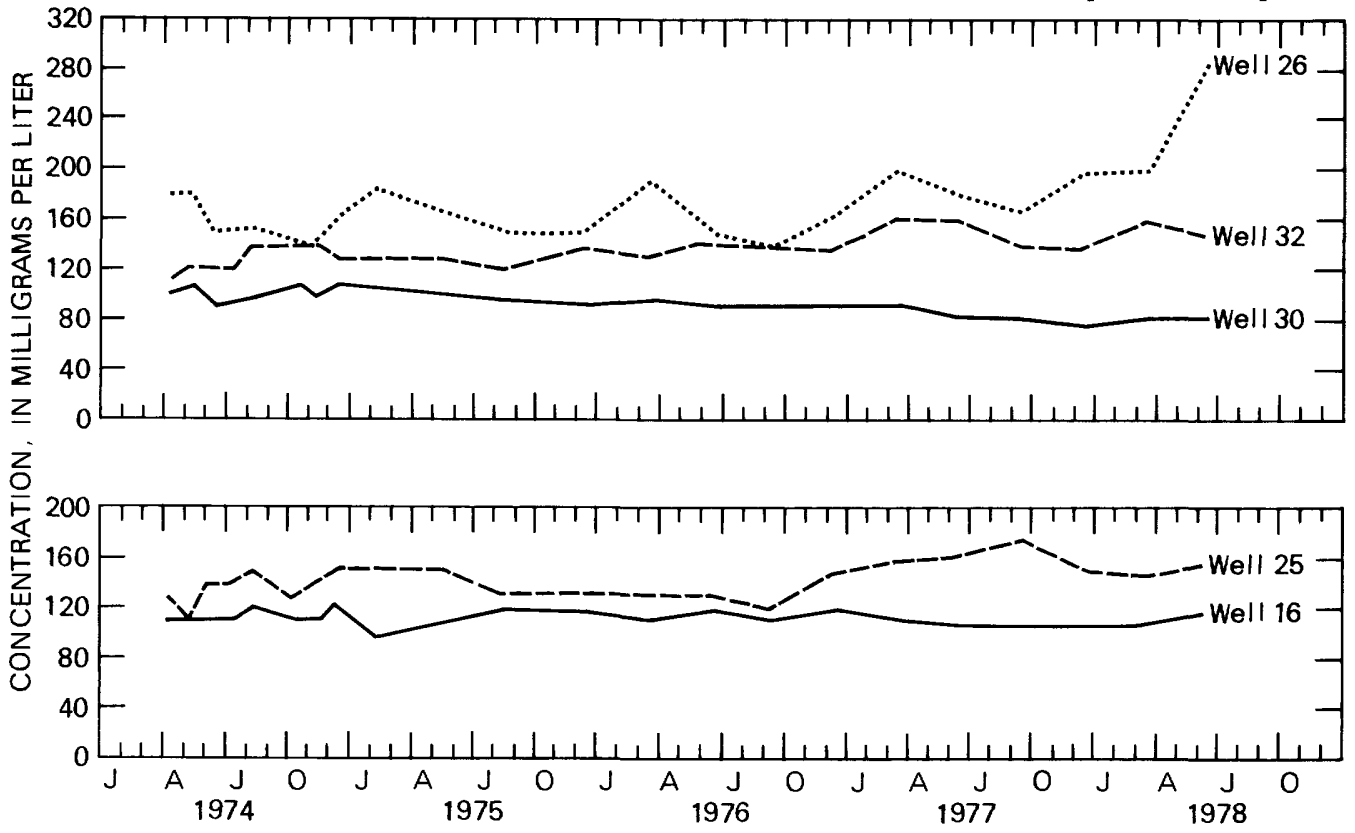

Figure 9.-- Chloride concentrations in water from selected wells in and near the feedlot.

Figure 4 and table 4 show well depths and the intervals of the wells open to the aquifer

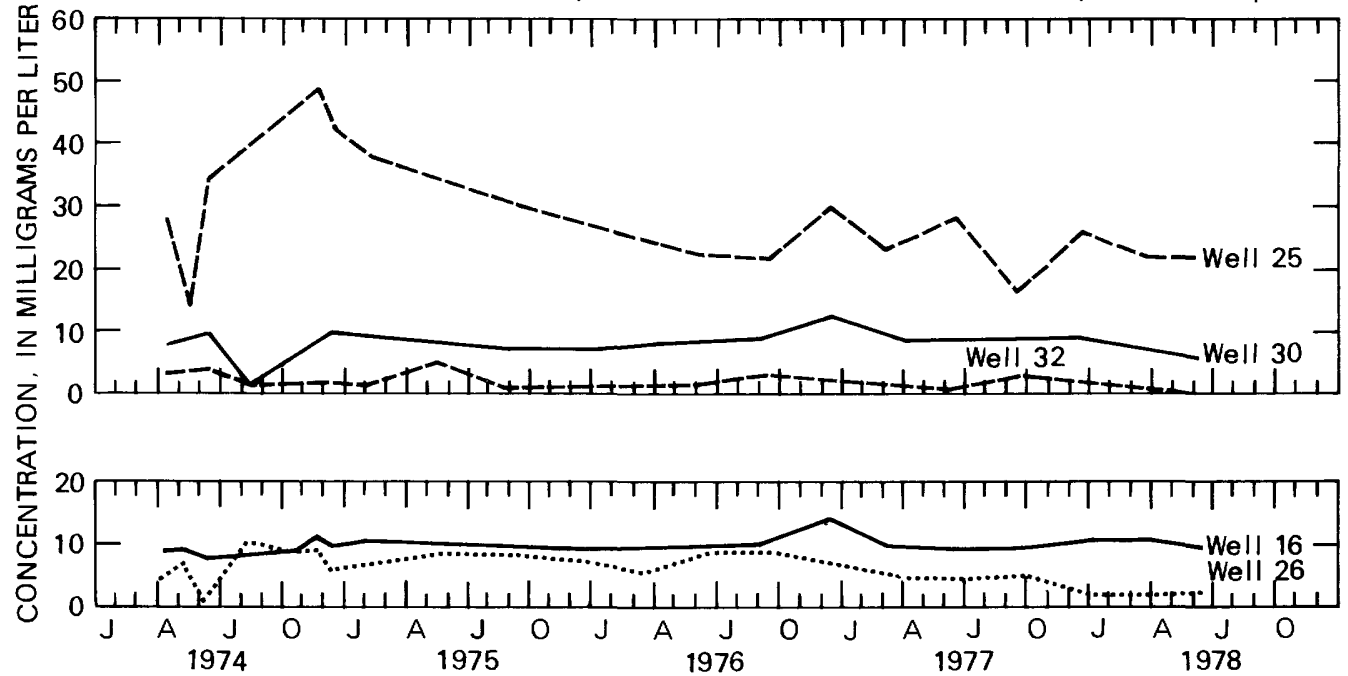

Figure 10. -- Nitrate concentrations in water from selected wells in and near the feedlot. 
Figure 4 and table 4 show well depths and the intervals of the wells open to the aquifer

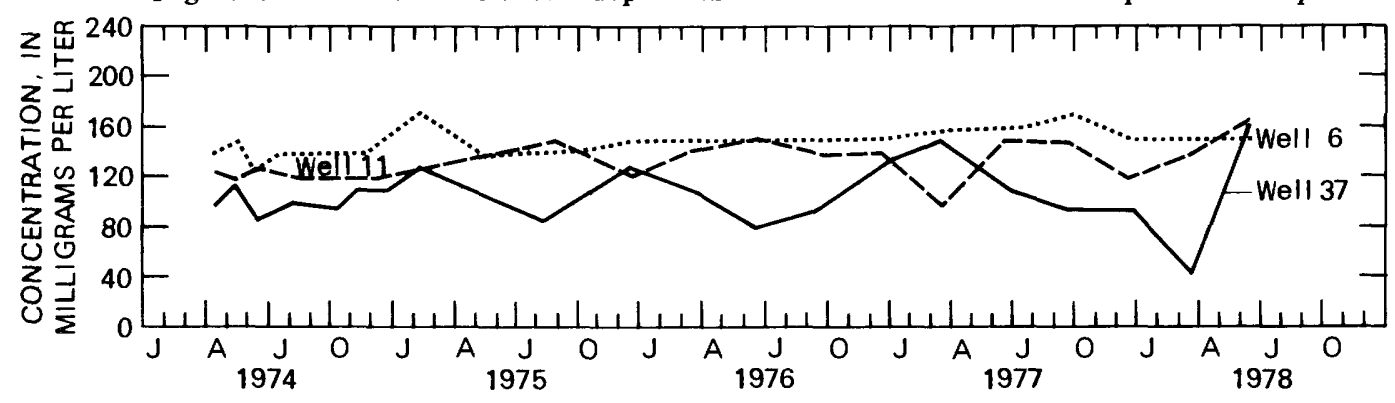

Figure 11.-- Chloride concentrations in water from selected wells out side the feedlot.

Figure 4 and table 4 show well depths and the intervals of the wells open to the aquifer

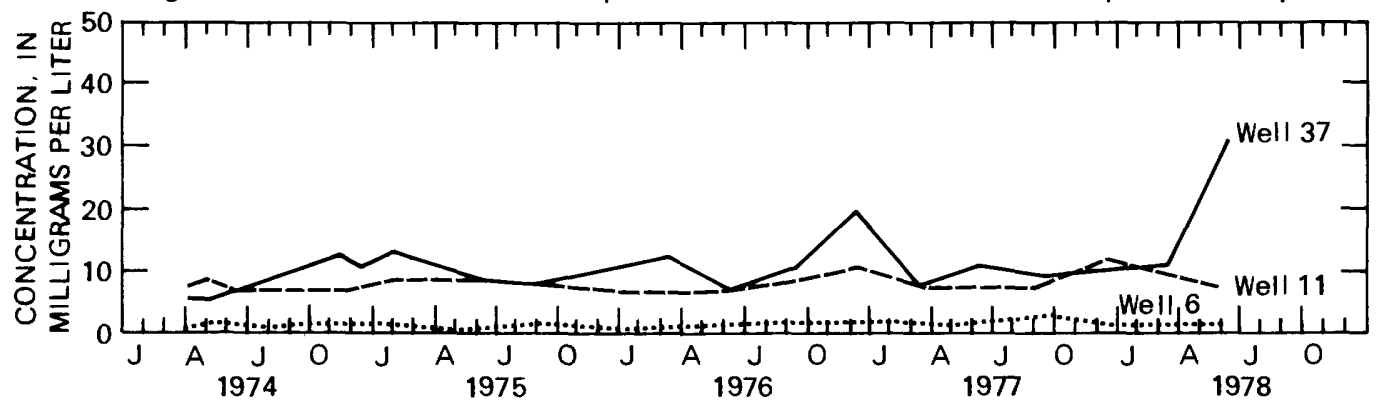

Figure 12. -- Nitrate concentrations in water from selected wells outside the feedlot.

Water samples from lysimeters installed at 5- and 20-ft depths below land surface in the unsaturated zone near wells 13, 14, 15, and 16 in the feedlot indicate that leachate from the feedlot surface has percolated to a depth of at least $5 \mathrm{ft}$ but not to a depth of $20 \mathrm{ft}$ (table 3). Lysimeter samples first obtained in July 1975 indicate leachate from the feedlot was present in the soil moisture at a depth of $5 \mathrm{ft}$ below land surface. The small amount of water obtained from the shallower lysimeter had a nitrite plus nitrate concentration of $230 \mathrm{mg} / \mathrm{L}$ as $\mathrm{nitrogen}$ and a chloride concentration of $210 \mathrm{mg} / \mathrm{L}$. Water from the deeper lysimeter had a nitrite plus nitrate concentration of $7.2 \mathrm{mg} / \mathrm{L}$ as $n i t r o g e n$ and a chloride concentration of $42 \mathrm{mg} / \mathrm{L}$. The concentrations of nitrite plus nitrate and chloride have not changed significantly from July 1975 to June 1978. 
Factors that may be responsible for the small changes in water quality in the alluvial aquifer in the study area, despite large quantities of wastes generated in the feedlot, include limited recharge from the feedlot because of a relatively impermeable manure pack and soil clogging under the pens, resulting in slow vertical movement of leachate in the unsaturated zone, soil clogging under the runoff-retention ponds, and denitrification in the unsaturated zone. The relatively small amount of precipitation and large potential evaporation (fig. 13) mean that little water is available for recharge. Cattle wastes and water sprinkled on the pens to control dust are potential sources of recharge. However, the total of all sources of recharge is relatively small in comparison to the quantity of water moving beneath the feedlot.

Data from U.S. Department of Commerce, National Weather Service

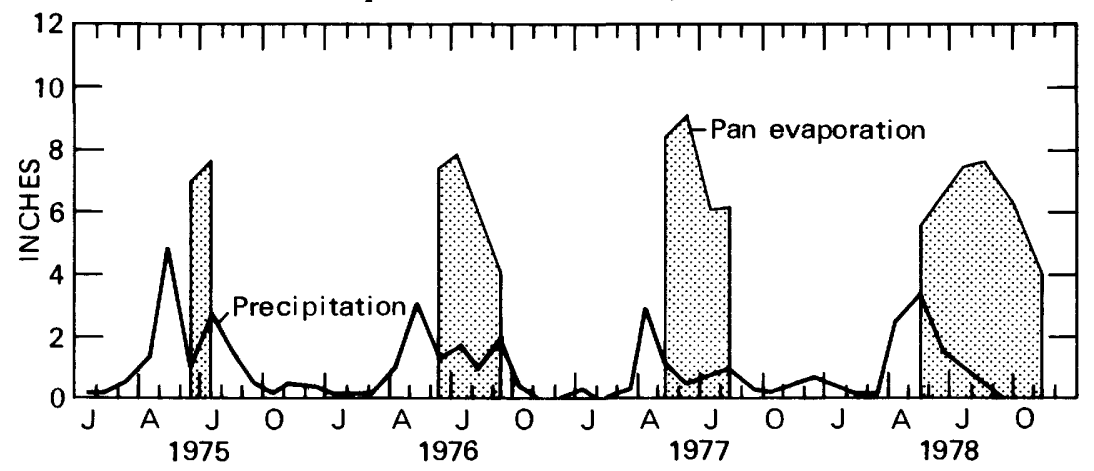

Figure 13. -- Monthly precipitation at the feedlot and pan evaporation at Fort Collins.

Infiltration to the unsaturated zone beneath the pens is inhibited by the relatively impermeable manure pack. The infiltration rate of undisturbed complete feedlot soil cores was reported by Mielke and Mazurak (1976, p. 344) to range from $1.2 \times 10^{-4} \mathrm{ft} / \mathrm{d}$ to $7.5 \times 10^{-4} \mathrm{ft} / \mathrm{d}$. Clogging of pore spaces by organic material also occurs beneath the pens. Manure packs that are unused, however, dry and crack, thus opening the manure pack and the surrounding area to water and oxygen. Such conditions promote leaching of nitrate down the soil profile to the water table (Mielke and Ellis, 1976, p. 74).

Observation indicates that leakage from the unlined runoff-retention ponds may be restricted because of soil clogging by suspended material in the runoff water. Schuman and McCalla (1975, p. 115) also reported that feedlot lagoon water applied to soil cores caused clogging. When water levels in the ponds decline, the clogged soil dries, cracks, and may allow some leakage as water levels in the ponds rise and before clogging reoccurs. 
Table 3.--Chemical analyses of

\begin{tabular}{|c|c|c|c|c|c|c|c|c|c|}
\hline 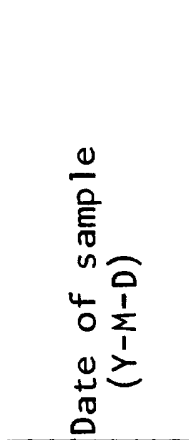 & 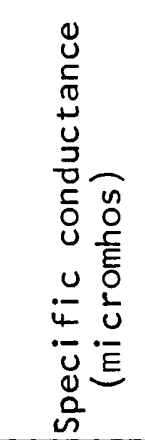 & $\begin{array}{l}\frac{n}{ \pm} \\
\frac{1}{5} \\
3 \\
x \\
a\end{array}$ & 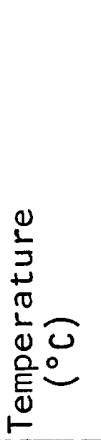 & 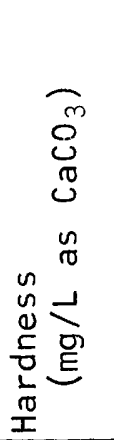 & 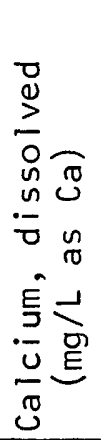 & 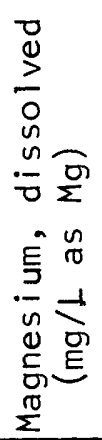 & 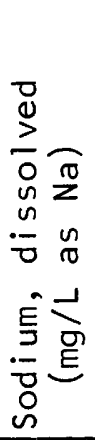 & 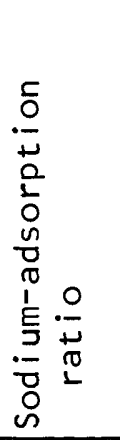 & 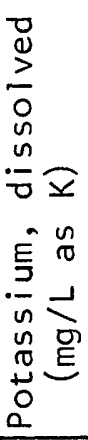 \\
\hline \multicolumn{10}{|c|}{ 5-FT LYSIMETER } \\
\hline $75-07-23$ & 4,500 & 7.4 & 27.0 & ---- & --- & --- & --- & ---- & --- \\
\hline $76-06-29$ & 5,000 & 7.2 & 12.5 & ---- & -- & --2 & --- & ---- & -- \\
\hline $76-09-22$ & --- & -- & ---- & ---- & --- & --- & --- & ---- & -- \\
\hline $76-12-08$ & 13,720 & -- & ---- & ---- & --- & --- & --- & ---- & --- \\
\hline $77-03-08$ & 13,750 & -- & ---- & $-\cdots$ & -- & --- & 320 & ---- & 14 \\
\hline $77-06-15$ & --- & $\ldots$ & $\ldots$ & 2,100 & 600 & 140 & 320 & 3.1 & 15 \\
\hline $77-09-13$ & 14,274 & -- & ---- & ---- & -- & --- & -- & $\cdots--$ & -- \\
\hline $77-12-06$ & ---- & --- & ---- & $-\cdots$ & --- & --- & --- & ---- & -- \\
\hline $78-03-09$ & 14,059 & 7.1 & ---- & --- & --- & --- & --- & ---- & --- \\
\hline $78-06-20$ & ---- & -- & $-\cdots$ & ---- & --- & --- & --- & ---- & -- \\
\hline \multicolumn{10}{|c|}{ 20-FT LYSIMETER } \\
\hline $75-07-23$ & 1,500 & 7.5 & 23.0 & ---- & --- & --- & --- & --- & -- \\
\hline $76-06-29$ & 1,700 & 6.9 & 12.0 & $-\ldots$ & -- & --- & -- & ---- & -- \\
\hline $76-09-22$ & ---- & --- & ---- & ---- & --- & --- & --- & ---- & --- \\
\hline $76-12-08$ & ${ }^{1} 1,650$ & -- & ---- & $-\cdots$ & --- & --- & --- & ---- & --- \\
\hline $77-03-08$ & ---- & -- & ---- & $-\cdots$ & --- & --- & 81 & --- & 18 \\
\hline $77-06-15$ & --- & 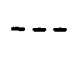 & --- & 990 & 290 & 65 & 92 & 1.3 & 18 \\
\hline $77-09-13$ & ${ }^{1} 1,953$ & $\cdots$ & --- & $\ldots-$ & -- & --- & --- & --- & --- \\
\hline $77-12-06$ & --- & --- & ---- & ---- & --- & --- & --- & --- & -- \\
\hline $78-06-20$ & ---- & --- & ---- & ---- & --- & --- & --- & ---- & --- \\
\hline
\end{tabular}

${ }^{I}$ Laboratory value. 
water from two lysimeters

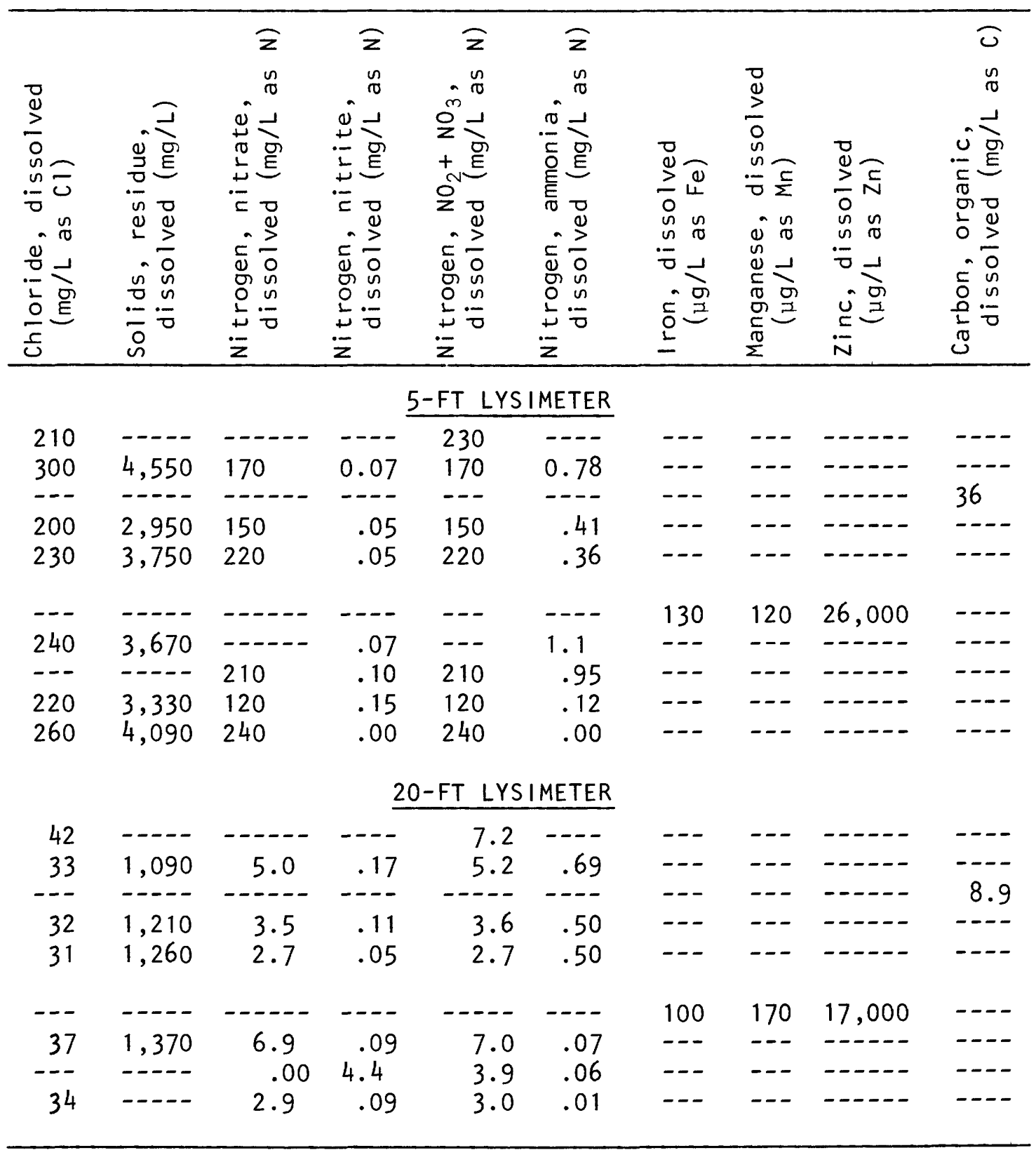


A relatively slow rate of vertical movement of leachate may mean that not enough time has elapsed for leachate to reach the saturated zone. The rate of vertical movement of water in the unsaturated zone under fields and animal pens in the middle South Platte Valley of Colorado has been estimated to be always less than $4 \mathrm{ft} / \mathrm{yr}$ and generally less than $1.2 \mathrm{ft} / \mathrm{yr}$ ( $S$ tewart and others, 1967a, p. 46).

Future changes in ground-water quality beneath the feedlot are likely to be minimal even when leachate reaches the saturated zone. The volume of water in the unsaturated zone reaching the water table is small, compared with the volume of ground water moving beneath the feedlot. Consequently, the concentrations of chemical constituents in the leachate will be diluted on reaching the saturated zone. Denitrification occurring in the soil profile also will lessen the impact of leachate on the concentration of nitrates in ground water. Concentrations of nitrite and nitrate nitrogen in water in the unsaturated zone under the feedlot should be larger than the potential of $540 \mathrm{mg} / \mathrm{L}$ calculated for ground water because of smaller volumes of water in the unsaturated zone. Water samples from the 5-ft lysimeter, however, averaged $190-\mathrm{mg} / \mathrm{L}$ nitrite and nitrate nitrogen for seven analyses, indicating denitrification is occurring in the soil profile under the manure pack.

\section{SUMMARY}

Water samples collected from 19 observation wells from April 1974, before the feedlot was stocked, to June 1978, when 90,000 cattle were fed, have indicated that few water-quality changes can be attributed to the feedlot despite the large quantities of generated waste. Water analyses from two lysimeters in the unsaturated zone indicate leachate from the feedlot has percolated to a depth of at least $5 \mathrm{ft}$ but has not percolated to a depth of $20 \mathrm{ft}$. The small changes in ground-water quality caused by the feedlot are likely due to the limited available recharge, a relatively impermeable manure pack, soil clogging under the cattle pens resulting in slow vertical movement of leachate through the unsaturated zone, soil clogging under the unlined runoff-retention ponds, and denitrification in the unsaturated zone.

\section{SELECTED REFERENCES}

Ciravolo, T. G., Martens, D. C., Hallock, D. L., Collins, E. R., Jr., Kornegag, E. T., and Thomas, H. R., 1979, Pollutant movement to shallow ground water tables from anaerobic swine waste lagoons: Journal of Envi ronmental Quality, v. 8, no. 1, p. 126-130.

Crosby, J. W., III, Johnstone, D. L., and Fenton, R. L., 1971, Migration of pollutants in a glacial outwash environment: Water Resources Research, v. 7 , no. 1, p. 204-208.

Elliott, L. F., McCalla, T. M., Mielke, L. N., and Travis, T. A., 1972, Ammonium, nitrate, and total nitrogen in the soil water of feedlot and field soil profiles: Applied Microbiology, v. 28, no. 4, p. 810-813. 
Gillham, R. W., and Webber, L. R., 1969, Nitrogen contamination of groundwater by barnyard leachates: Water Pollution Control Federation Journal, v. 41 , no. 10 , p. 1752-1762.

Hurr, R. T., and Schneider, P. A., Jr., 1972, Hydrogeologic characteristics of the valley-fill aquifer in the Greeley reach of the South Platte River valley, Colorado: U.S. Geological Survey Open-File report, 2 p., 6 maps.

Lorimor, J. C., Mielke, L. N., Elliott, L. F., and Ellis, J. R., 1972, Nitrate concentrations in groundwater beneath a beef cattle feedlot: Water Resources Bulletin, v. 8, no. 5, p. 999-1005.

McCalla, T. M., Ellis, J. R., Gilbertson, C. B., and Woods, W. R., 1972, Chemical studies of solids, runoff, soil profile and groundwater from beef cattle feedlots at Mead, Nebraska: Cornell University Agricultural Waste Management Conference, Syracuse, N.Y., 1972, Proceedings, p. 211223.

McCalla, T. M., Ellis, J. R., and Woods, W. R., 1969, Changes in the chemical and biological properties of beef cattle manure during decomposition: Bacteriological Proceedings, p. 4-5.

Mielke, L. N., and Ellis, J.R., 1976, Nitrogen in soil cores and ground water under abandoned cattle feedlots: Journal of Environmental Quality, v. 5, no. 1, p. 71-74.

Mielke, L. N., Ellis, J. R., Swanson, N. P., Lorimor, J. C., and McCalla, T. M., 1970, Groundwater quality and fluctuations in a shallow unconfined aquifer under a level feedlot: Cornell University Agricultural Waste Management Conference, Rochester, N.Y., 1970, Proceedings, p. $31-40$.

Mielke, L. N., and Mazurak, A. P., 1976, Infiltration of water on a cattle feedlot: Transactions of the American Society of Agricultural Engineers, v. 19, no. 2, p. 341-344.

Mosier, A. R., Haider, K., and Clark, F. E., 1972, Water soluble organic substances leachable from feedlot manure: Journal of Environmental Quality, v. 1 , no. 3, p. 320-322.

Robson, S. G., 1977, Ground-water quality near a sewage-sludge recycling site and a landfill near Denver, Colorado: U.S. Geological Survey WaterResources Investigations $76-132,137$ p.; available from U.S. Department of Commerce, National Technical Information Service, Springfield, VA 22161 , as report PB-269 294/AS.

Schuman, G. E., and McCalla, T. M., 1975, Chemical characteristics of a feedlot soil profile: Soil Science, v. 119, no. 2, p. 113-118.

Stewart, B. A., Viets, F. G., Jr., Hutchinson, G. L., and Kemper, W. D., 1967a, Nitrate and other water pollutants under fields and feedlots: Environmental Science and Technology, v. 1, no. 9, p. 736-739.

Stewart, B. A., Viets, F. G., Jr., Hutchinson, G. L., Kemper, W. D., Clark, F. E., Fairbourn, M. L., and Strauch, F., 1967b, Distribution of nitrates and other water pollutants under fields and corrals in the middle South Platte Valley of Colorado: U.S. Department of Agriculture, Agricultural Research Service, 41-134, 206 p.

Taiganides, E. P., and Hazen, T. E., 1966, Properties of farm animal excreta: American Society of Agricultural Engineers Transactions, v. 9, no. 3, p. $374-376$. 
Texas Tech University, 1971, Infiltration rates and ground-water quality beneath cattle feedlots, Texas High Plains: U.S. Environmental Protection Agency, Water Pollution Control Research Series 16060 EGS 01/71, $55 \mathrm{p}$.

Wood, W. W., 1973, A technique using porous cups for water sampling at any depth in the unsaturated zone: Water Resources Research, v. 9, no. 2, p. $486-488$. 
SUPPLEMENTAL INFORMATION 
System of Numbering Wells Using Township, Range, and Sections

The system of numbering wells using township, range, and section is illustrated in figure 14. The well numbers used in tables 4 to 10 are based on this numbering system. 


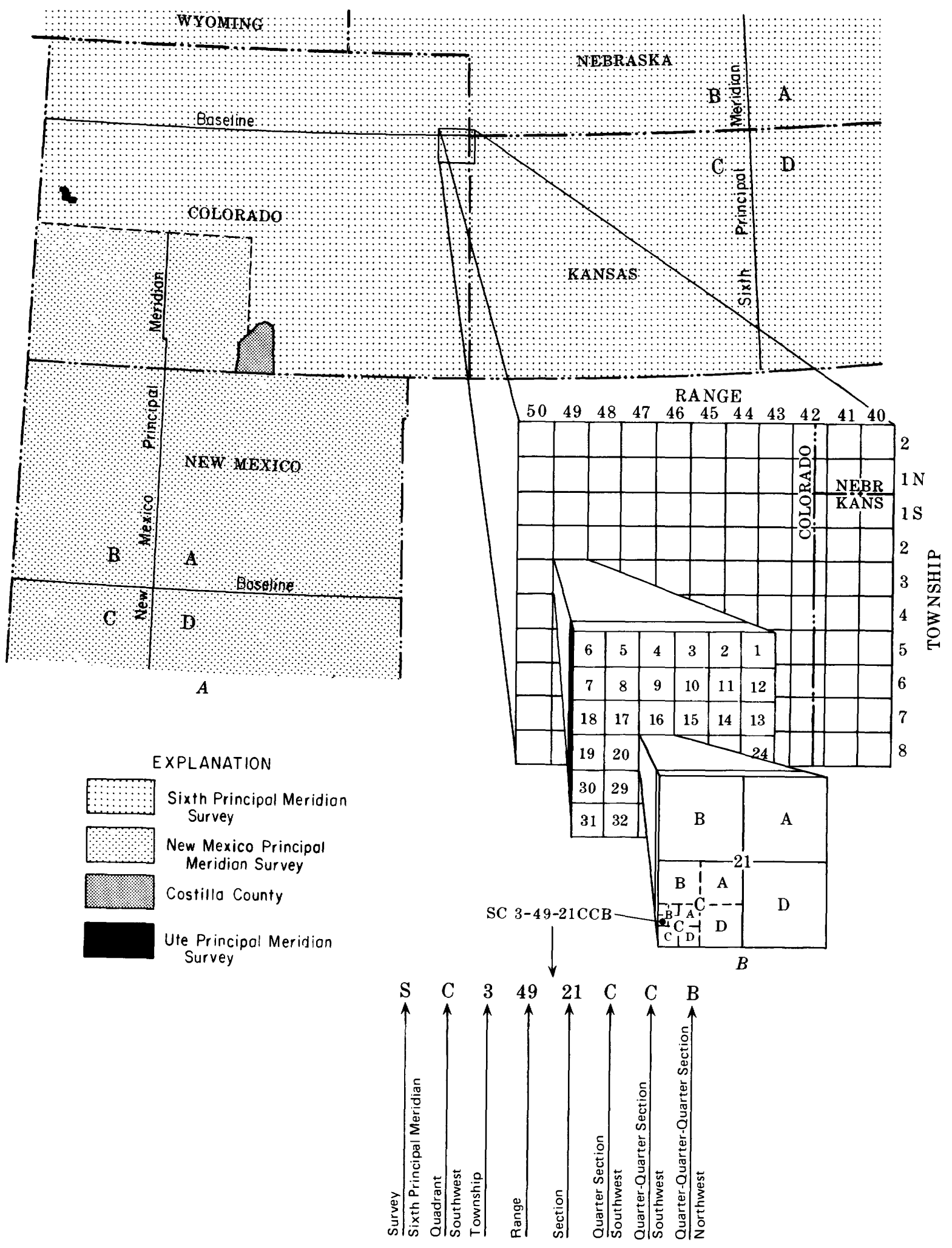

Figure 14. -- System of numbering wells using town ship, range, and section. 


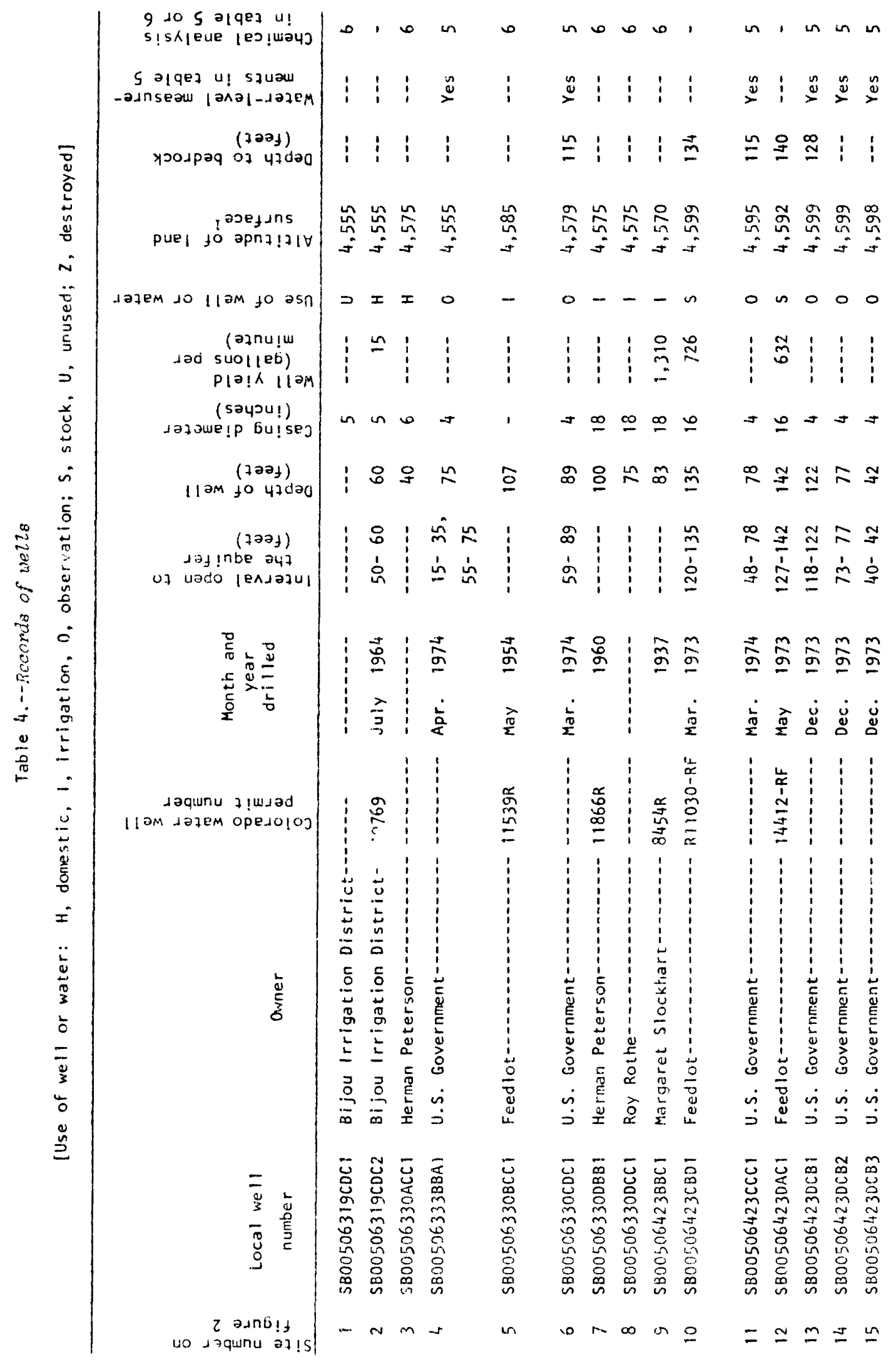




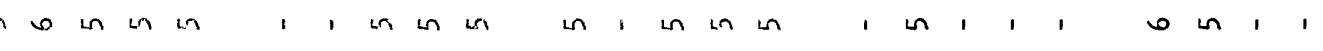

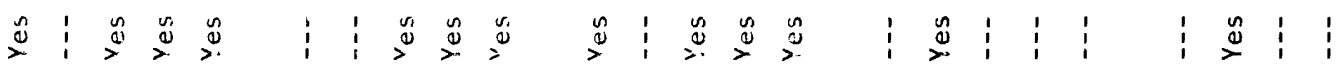
1: $1:$ 자:

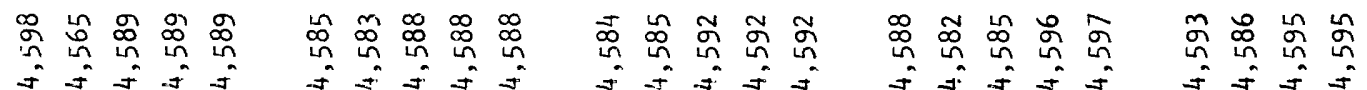

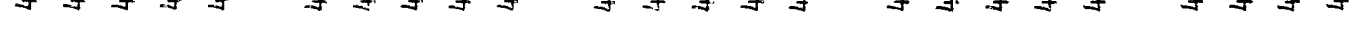

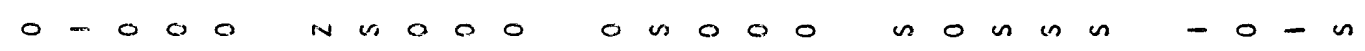

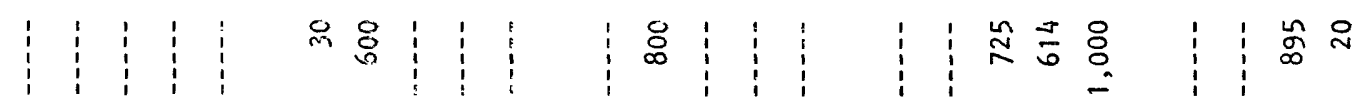

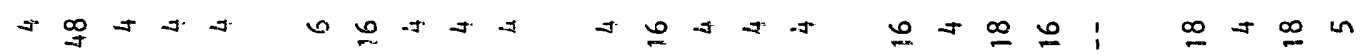
요윰

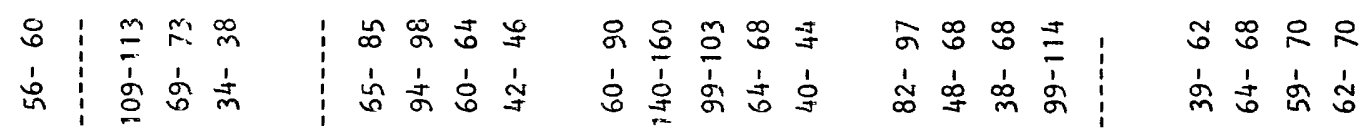

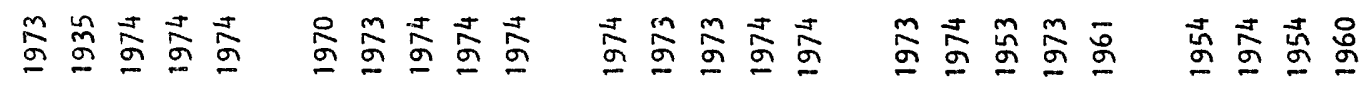

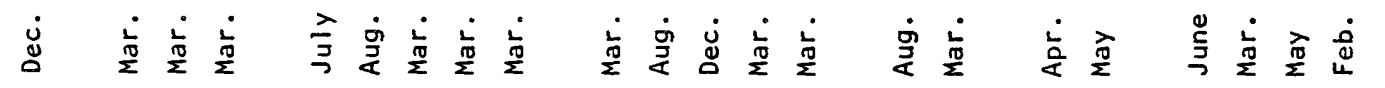
:
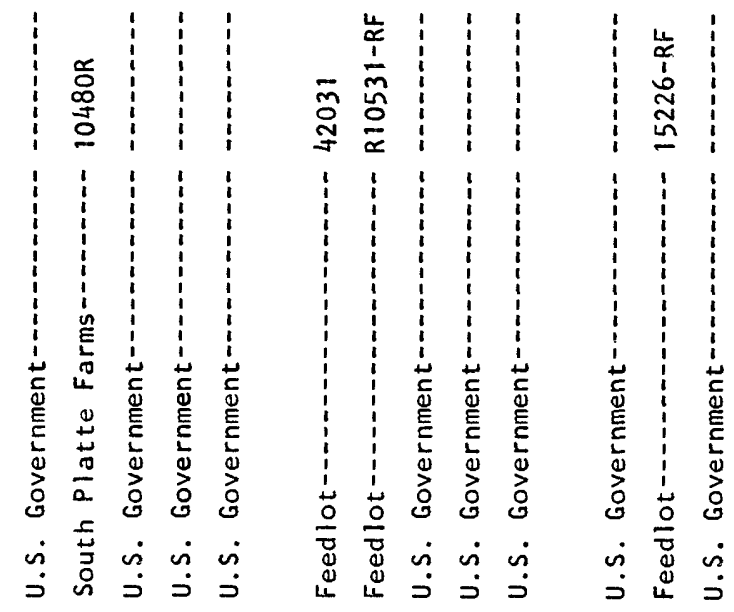

:
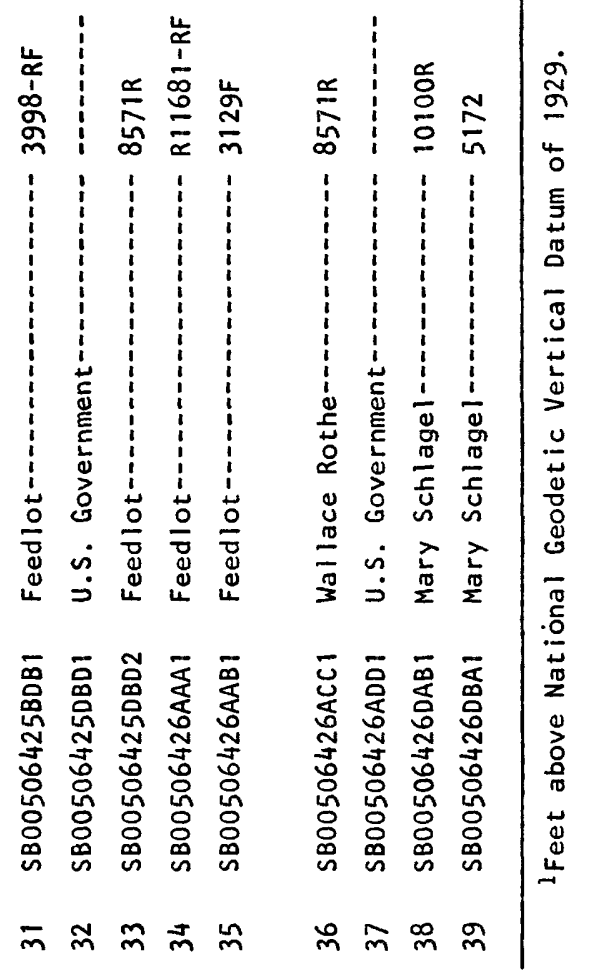
Table 5.--Water-Zevel records and chemical [Wel1 number 4 on figure 2. Local well number is SB00506333BBA1. Depth

\begin{tabular}{|c|c|c|c|c|c|c|c|c|}
\hline $\begin{array}{c}\text { DATE OF } \\
\text { WATER-LEVEL } \\
\text { MEASUREMENT }\end{array}$ & $\begin{array}{l}\text { WATER } \\
\text { LEVEL } \\
\text { (FEET } \\
\text { BELOW } \\
\text { LAND } \\
\text { SURFACE) }\end{array}$ & $\begin{array}{c}\text { DATE } \\
\text { OF } \\
\text { SANPLE }\end{array}$ & $\begin{array}{l}\text { SPE- } \\
\text { CIFIC } \\
\text { CON- } \\
\text { DUCT- } \\
\text { ANCE } \\
\text { (MICRO- } \\
\text { MHOS) }\end{array}$ & $\begin{array}{c}\text { PH } \\
\text { (UNITS) }\end{array}$ & $\begin{array}{l}\text { TEMPER- } \\
\text { ATURE } \\
\text { (DEG C) }\end{array}$ & $\begin{array}{l}\text { HARD- } \\
\text { NESS } \\
\text { (MG/L } \\
\text { AS } \\
\text { CACO3) }\end{array}$ & $\begin{array}{l}\text { HARD- } \\
\text { NESS, } \\
\text { NONCAR- } \\
\text { BONATE } \\
\text { (MG/L } \\
\text { CACO3) }\end{array}$ & $\begin{array}{c}\text { CALCIUM, } \\
\text { DIS- } \\
\text { SOLVED } \\
\text { (MG/L } \\
\text { AS CA) }\end{array}$ \\
\hline $\begin{array}{l}74-04-04 \\
74-05-08 \\
74-06-14 \\
74-07-23 \\
74-08-27\end{array}$ & $\begin{array}{l}4.55 \\
5.03 \\
4.34 \\
4.93 \\
4.84\end{array}$ & $\begin{array}{l}74-04-04 \\
74-05-09 \\
74-06-14 \\
74-07-24 \\
74-08-28\end{array}$ & $\begin{array}{l}2190 \\
2190 \\
2190 \\
2190 \\
2170\end{array}$ & $\begin{array}{r}7.2 \\
7.4 \\
7.5 \\
7.5 \\
--\end{array}$ & $\begin{array}{l}12.5 \\
13.0 \\
13.5 \\
14.5 \\
13.5\end{array}$ & $\begin{array}{l}860 \\
830 \\
800 \\
820 \\
860\end{array}$ & $\begin{array}{l}540 \\
510 \\
480 \\
500 \\
540\end{array}$ & $\begin{array}{l}210 \\
200 \\
190 \\
200 \\
210\end{array}$ \\
\hline $\begin{array}{l}74-10-03 \\
74-11-11 \\
74-12-05 \\
75-02-06 \\
76-12-08\end{array}$ & $\begin{array}{l}4.74 \\
4.78 \\
4.93 \\
4.86 \\
4.40\end{array}$ & $\begin{array}{l}74-10-04 \\
74-11-11 \\
74-12-05 \\
75-02-06 \\
76-12-08\end{array}$ & $\begin{array}{l}2160 \\
2150 \\
2400 \\
2300 \\
2100\end{array}$ & $\begin{array}{l}-- \\
7.2 \\
6.8 \\
7.1 \\
7.5\end{array}$ & $\begin{array}{l}13.5 \\
13.0 \\
13.0 \\
12.5 \\
13.5\end{array}$ & $\begin{array}{l}850 \\
770 \\
840 \\
830 \\
840\end{array}$ & $\begin{array}{l}520 \\
450 \\
520 \\
510 \\
530\end{array}$ & $\begin{array}{l}210 \\
200 \\
210 \\
200 \\
200\end{array}$ \\
\hline $\begin{array}{l}77-03-08 \\
77-06-15 \\
77-09-13 \\
77-12-06 \\
78-03-09\end{array}$ & $\begin{array}{l}4.71 \\
5.35 \\
4.90 \\
4.46 \\
4.65\end{array}$ & $\begin{array}{l}77-03-08 \\
77-06-15 \\
77-09-13 \\
77-12-06 \\
78-03-09\end{array}$ & $\begin{array}{l}2400 \\
2400 \\
2200 \\
2200 \\
2400\end{array}$ & $\begin{array}{l}7.6 \\
7.5 \\
7.4 \\
7.3 \\
7.4\end{array}$ & $\begin{array}{l}13.5 \\
14.5 \\
13.5 \\
12.5 \\
13.0\end{array}$ & $\begin{array}{l}850 \\
860 \\
880 \\
870 \\
920\end{array}$ & $\begin{array}{l}530 \\
550 \\
560 \\
540 \\
600\end{array}$ & $\begin{array}{l}210 \\
210 \\
210 \\
210 \\
220\end{array}$ \\
\hline $78-06-20$ & 3.84 & $78-06-20$ & 2300 & 7.4 & 13.5 & 910 & 570 & 220 \\
\hline $\begin{array}{l}\text { DATE OF } \\
\text { WATER-LEVEL } \\
\text { MEASUREMENT }\end{array}$ & $\begin{array}{l}\text { WATER } \\
\text { LEVEL } \\
\text { (FEET } \\
\text { BELOW } \\
\text { LAND } \\
\text { SURFACE) }\end{array}$ & $\begin{array}{c}\text { DATE } \\
\text { OF } \\
\text { SAMPLE }\end{array}$ & $\begin{array}{l}\text { FLUO- } \\
\text { RIDE, } \\
\text { DIS- } \\
\text { SOLVED } \\
\text { (MG/L } \\
\text { AS F) }\end{array}$ & $\begin{array}{c}\text { SILICA, } \\
\text { DIS- } \\
\text { SOLVED } \\
\text { (MG/L } \\
\text { AS } \\
\text { SI02) }\end{array}$ & $\begin{array}{l}\text { SOLIDS, } \\
\text { SUM OF } \\
\text { CONSTI- } \\
\text { TUENTS, } \\
\text { DIS- } \\
\text { SOLVED } \\
\text { (MG/L) }\end{array}$ & $\begin{array}{l}\text { NITRO- } \\
\text { GEN, } \\
\text { NITRATE } \\
\text { DIS- } \\
\text { SOLVED } \\
\text { (MG/L } \\
\text { AS N) }\end{array}$ & $\begin{array}{l}\text { NITRO- } \\
\text { GEN, } \\
\text { NITRITE } \\
\text { DIS- } \\
\text { SOLVED } \\
\text { (MG/L } \\
\text { AS N) }\end{array}$ & $\begin{array}{l}\text { NITRO- } \\
\text { GEN, } \\
\text { N02+NO3 } \\
\text { DIS- } \\
\text { SOLVED } \\
\text { (MG/L } \\
\text { AS N) }\end{array}$ \\
\hline $\begin{array}{l}74-04-04 \\
74-05-08 \\
74-06-14 \\
74-07-23 \\
74-08-27\end{array}$ & $\begin{array}{l}4.55 \\
5.03 \\
4.34 \\
4.93 \\
4.84\end{array}$ & $\begin{array}{l}74-04-04 \\
74-05-09 \\
74-06-14 \\
74-07-24 \\
74-08-28\end{array}$ & $\begin{array}{l}1.4 \\
1.3 \\
1.2 \\
1.3 \\
1.3\end{array}$ & $\begin{array}{l}17 \\
17 \\
20 \\
17 \\
17\end{array}$ & $\begin{array}{l}1630 \\
1570 \\
1500 \\
1580 \\
1590\end{array}$ & $\begin{array}{r}5.2 \\
6.5 \\
4.7 \\
-. \\
--\end{array}$ & $\begin{array}{l}.51 \\
.02 \\
.01 \\
-- \\
.01\end{array}$ & $\begin{array}{l}5.7 \\
6.5 \\
4.7 \\
6.0 \\
5.9\end{array}$ \\
\hline $\begin{array}{l}74-10-03 \\
74-11-11 \\
74-12-05 \\
75-02-06 \\
76-12-08\end{array}$ & $\begin{array}{l}4.74 \\
4.78 \\
4.93 \\
4.86 \\
4.40\end{array}$ & $\begin{array}{l}74-10-04 \\
74-11-11 \\
74-12-05 \\
75-02-06 \\
76-12-08\end{array}$ & $\begin{array}{l}1.3 \\
1.3 \\
1.3 \\
1.2 \\
1.3\end{array}$ & $\begin{array}{l}17 \\
17 \\
16 \\
17 \\
16\end{array}$ & $\begin{array}{l}1630 \\
1490 \\
1570 \\
1630 \\
1650\end{array}$ & $\begin{array}{l}6.7 \\
5.7 \\
6.6 \\
9.4\end{array}$ & $\begin{array}{l}.00 \\
.00 \\
.00 \\
.00 \\
.01\end{array}$ & $\begin{array}{l}5.8 \\
6.7 \\
5.7 \\
6.6 \\
9.4\end{array}$ \\
\hline $\begin{array}{l}77-03-08 \\
77-06-15 \\
77-09-13 \\
77-12-06 \\
78-03-09\end{array}$ & $\begin{array}{l}4.71 \\
5.35 \\
4.90 \\
4.46 \\
4.65\end{array}$ & $\begin{array}{l}77-03-08 \\
77-06-15 \\
77-09-13 \\
77-12-06 \\
78-03-09\end{array}$ & $\begin{array}{l}1.4 \\
1.3 \\
1.3 \\
1.3 \\
1.4\end{array}$ & $\begin{array}{l}18 \\
16 \\
17 \\
17 \\
16\end{array}$ & $\begin{array}{l}1610 \\
1650 \\
1660 \\
1650 \\
1680\end{array}$ & $\begin{array}{l}6.7 \\
7.2 \\
7.2 \\
8.3 \\
8.1\end{array}$ & $\begin{array}{l}.01 \\
.00 \\
.00 \\
.00 \\
.01\end{array}$ & $\begin{array}{l}6.7 \\
7.2 \\
7.2 \\
8.3 \\
8.1\end{array}$ \\
\hline $78-06-20$ & 3.84 & $78-06-20$ & 1.2 & 14 & 1650 & 7.0 & .02 & 7.0 \\
\hline
\end{tabular}


analyses of water from observation wells

is $75 \mathrm{ft}$. Intervals open to the aquifer is 15 to $35 \mathrm{ft}$ and 55 to $75 \mathrm{ft}$ ]

\begin{tabular}{|c|c|c|c|c|c|c|c|c|}
\hline $\begin{array}{l}\text { MAGNE- } \\
\text { SIUM, } \\
\text { DIS- } \\
\text { SOLVED } \\
\text { (MG/L } \\
\text { AS MG) }\end{array}$ & $\begin{array}{l}\text { SODIUM, } \\
\text { DIS- } \\
\text { SOLVED } \\
\text { (MG/L } \\
\text { AS NA) }\end{array}$ & $\begin{array}{c}\text { SODIUM } \\
\text { AD- } \\
\text { SORP- } \\
\text { TION } \\
\text { RATIO }\end{array}$ & $\begin{array}{l}\text { POTAS- } \\
\text { SIUM, } \\
\text { DIS- } \\
\text { SOLVED } \\
\text { (MG/L } \\
\text { AS K) }\end{array}$ & $\begin{array}{c}\text { BICAR- } \\
\text { BONATE } \\
\text { (MG/L } \\
\text { AS } \\
\text { HCO3) }\end{array}$ & $\begin{array}{c}\text { CAR- } \\
\text { BONATE } \\
\text { (MG/L } \\
\text { AS CO3) }\end{array}$ & $\begin{array}{c}\text { ALKA- } \\
\text { LINITY } \\
\text { (MG/L } \\
\text { AS } \\
\text { CAC03) }\end{array}$ & $\begin{array}{c}\text { SULFATE, } \\
\text { DIS- } \\
\text { SOLVED } \\
\text { (MG/L } \\
\text { AS SO4) }\end{array}$ & $\begin{array}{l}\text { CHLC } \\
\text { RIDE } \\
\text { DIS- } \\
\text { SOLI } \\
\text { (MG/ } \\
\text { AS C }\end{array}$ \\
\hline $\begin{array}{l}81 \\
80 \\
79 \\
77 \\
82\end{array}$ & $\begin{array}{l}210 \\
200 \\
200 \\
210 \\
200\end{array}$ & $\begin{array}{l}3.1 \\
3.0 \\
3.1 \\
3.2 \\
3.0\end{array}$ & $\begin{array}{l}5.2 \\
5.2 \\
4.9 \\
5.1 \\
6.0\end{array}$ & $\begin{array}{l}393 \\
389 \\
386 \\
389 \\
388\end{array}$ & $\begin{array}{c}0 \\
0 \\
0 \\
-- \\
--\end{array}$ & $\begin{array}{l}322 \\
319 \\
317 \\
319 \\
318\end{array}$ & $\begin{array}{l}810 \\
760 \\
720 \\
770 \\
770\end{array}$ & $\begin{array}{l}79 \\
89 \\
77 \\
84 \\
87\end{array}$ \\
\hline $\begin{array}{l}78 \\
66 \\
77 \\
80 \\
83\end{array}$ & $\begin{array}{l}200 \\
210 \\
200 \\
210 \\
210\end{array}$ & $\begin{array}{l}3.0 \\
3.3 \\
3.0 \\
3.2 \\
3.2\end{array}$ & $\begin{array}{l}5.1 \\
3.3 \\
4.8 \\
5.0 \\
5.2\end{array}$ & $\begin{array}{l}400 \\
387 \\
391 \\
393 \\
385\end{array}$ & $\begin{array}{l}-- \\
-- \\
-- \\
0\end{array}$ & $\begin{array}{l}328 \\
317 \\
321 \\
322 \\
316\end{array}$ & $\begin{array}{l}810 \\
700 \\
760 \\
800 \\
820\end{array}$ & $\begin{array}{l}83 \\
73 \\
85 \\
92 \\
86\end{array}$ \\
\hline $\begin{array}{l}80 \\
81 \\
87 \\
84 \\
89\end{array}$ & $\begin{array}{l}210 \\
210 \\
220 \\
200 \\
190\end{array}$ & $\begin{array}{l}3.1 \\
3.1 \\
3.2 \\
3.0 \\
2.7\end{array}$ & $\begin{array}{l}5.1 \\
5.1 \\
4.9 \\
5.1 \\
5.2\end{array}$ & $\begin{array}{l}396 \\
380 \\
390 \\
400 \\
390\end{array}$ & $\begin{array}{l}0 \\
0 \\
0 \\
0 \\
0\end{array}$ & $\begin{array}{l}325 \\
312 \\
320 \\
330 \\
320\end{array}$ & $\begin{array}{l}770 \\
820 \\
810 \\
810 \\
800\end{array}$ & $\begin{array}{r}90 \\
88 \\
89 \\
85 \\
130\end{array}$ \\
\hline 88 & 210 & 3.0 & 5.2 & 420 & 0 & 340 & 780 & 07 \\
\hline
\end{tabular}
NITRO- NITRO- PHOS-
GEN, GEN,AM- PHORUS, MANGA-
AMMONIA MONIA + ORTHO, IRON, NESE,
DIS- ORGANIC DIS- DIS- DIS-
$\begin{array}{lcccc}\text { SOLVED } & \text { DIS. } & \text { SOLVED } & \text { SOLVED } & \text { SOLVED } \\ \text { (MG/L) } & (\mathrm{MG} / \mathrm{L} & \text { (MG/L } & \text { (UG/L } & \text { (UG/L }\end{array}$
$\begin{array}{lllll}(M G / L) & (M G / L & (M G / L & (U G / L & (U G / L \\ A S N) & A S N) & A S P) & \text { AS FE) } & \text { AS MN) }\end{array}$

$\begin{array}{rrrrr}.02 & .82 & .03 & 30 & 30 \\ .02 & .27 & .01 & 130 & 50 \\ .01 & .35 & .02 & 20 & 0 \\ .- & .- & .05 & 20 & 0 \\ .01 & .42 & .07 & 20 & 0 \\ .04 & .23 & .04 & 40 & 30 \\ .01 & .41 & .03 & 10 & 0 \\ .04 & .24 & .05 & 10 & 0 \\ .00 & .37 & .03 & 10 & 0 \\ .00 & .60 & .03 & 90 & 60 \\ .00 & .38 & .05 & 80 & 20 \\ .00 & .15 & .04 & 170 & 30 \\ .01 & .40 & .03 & 90 & 20 \\ .02 & .22 & .03 & 160 & 4 \\ .01 & .57 & .03 & 110 & 10 \\ .03 & 1.2 & .03 & 100 & 90\end{array}$


Table 5.--Water-level records and chemical [Well number 6 on figure 2. Local well number is SB00506330CDC1.

\begin{tabular}{|c|c|c|c|c|c|c|c|c|}
\hline $\begin{array}{l}\text { DATE OF } \\
\text { ATER-LEVEL } \\
\text { EAS'JREMENT }\end{array}$ & $\begin{array}{l}\text { WATER } \\
\text { LEVEL } \\
\text { (FEET } \\
\text { BELOW } \\
\text { LAND } \\
\text { SURFACE) }\end{array}$ & $\begin{array}{c}\text { DATE } \\
\text { OF } \\
\text { SAMPLE }\end{array}$ & $\begin{array}{l}\text { SPE- } \\
\text { CIFIC } \\
\text { CON- } \\
\text { DUCT- } \\
\text { ANCE } \\
\text { (MICRO- } \\
\text { MHOS) }\end{array}$ & $\begin{array}{c}\mathrm{PH} \\
\text { (UNITS) }\end{array}$ & $\begin{array}{l}\text { TEMPER- } \\
\text { ATURE } \\
\text { (DEG C) }\end{array}$ & $\begin{array}{l}\text { HARD- } \\
\text { NESS } \\
\text { (MG/L } \\
\text { AS } \\
\text { CACO3) }\end{array}$ & $\begin{array}{l}\text { HARD- } \\
\text { NESS, } \\
\text { NONCAR- } \\
\text { BONATE } \\
\text { (MG/L } \\
\text { CACO3) }\end{array}$ & $\begin{array}{l}\text { CALCIUi', } \\
\text { DIS- } \\
\text { SOLVED } \\
\text { (MG/L } \\
\text { AS CA) }\end{array}$ \\
\hline $\begin{array}{l}74-04-04 \\
74-04-08 \\
74-06-14 \\
74-07-23 \\
74-08-27\end{array}$ & $\begin{array}{l}26.58 \\
26.90 \\
23.78 \\
25.82 \\
25.43\end{array}$ & $\begin{array}{l}74-04-04 \\
74-05-09 \\
74-06-14 \\
74-07-24 \\
74-08-28\end{array}$ & $\begin{array}{l}2820 \\
2810 \\
2780 \\
2760 \\
2760\end{array}$ & $\begin{array}{l}7.3 \\
7.4 \\
7.4 \\
7.5 \\
--\end{array}$ & $\begin{array}{l}11.0 \\
11.5 \\
12.0 \\
13.0 \\
12.0\end{array}$ & $\begin{array}{r}1000 \\
1000 \\
1000 \\
970 \\
990\end{array}$ & $\begin{array}{l}640 \\
640 \\
620 \\
600 \\
620\end{array}$ & $\begin{array}{l}290 \\
290 \\
280 \\
270 \\
280\end{array}$ \\
\hline $\begin{array}{l}74-10-03 \\
74-11-11 \\
74-12-05 \\
75-02-06 \\
75-05-14\end{array}$ & $\begin{array}{l}24.82 \\
25.48 \\
26.00 \\
26.51 \\
25.88\end{array}$ & $\begin{array}{l}74-10-04 \\
74-11-11 \\
74-12-05 \\
75-02-06 \\
75-05-14\end{array}$ & $\begin{array}{l}2770 \\
2900 \\
3600 \\
3100 \\
3750\end{array}$ & $\begin{array}{l}7 .- \\
7.1 \\
7.1 \\
7.3\end{array}$ & $\begin{array}{l}11.5 \\
11.0 \\
12.0 \\
11.0 \\
12.5\end{array}$ & $\begin{array}{r}1100 \\
920 \\
1100 \\
1100 \\
1100\end{array}$ & $\begin{array}{l}720 \\
550 \\
680 \\
720 \\
700\end{array}$ & $\begin{array}{l}320 \\
250 \\
300 \\
310 \\
310\end{array}$ \\
\hline $\begin{array}{l}75-08-11 \\
75-12-02 \\
76-03-29 \\
76-06-28 \\
76-09-22\end{array}$ & $\begin{array}{l}25.49 \\
25.08 \\
26.24 \\
25.66 \\
24.71\end{array}$ & $\begin{array}{l}75-08-11 \\
75-12-02 \\
76-03-29 \\
76-06-28 \\
76-09-22\end{array}$ & $\begin{array}{l}3800 \\
3800 \\
3000 \\
2700 \\
3000\end{array}$ & $\begin{array}{l}7.5 \\
7.4 \\
7.5 \\
7.2 \\
7.1\end{array}$ & $\begin{array}{l}13.5 \\
12.5 \\
11.5 \\
12.5 \\
12.0\end{array}$ & $\begin{array}{l}1100 \\
1100 \\
1100 \\
1100 \\
1100\end{array}$ & $\begin{array}{l}740 \\
710 \\
680 \\
650 \\
720\end{array}$ & $\begin{array}{l}310 \\
310 \\
310 \\
300 \\
320\end{array}$ \\
\hline $\begin{array}{l}76-12-08 \\
77-03-08 \\
77-06-15 \\
77-09-13 \\
77-12-06\end{array}$ & $\begin{array}{l}25.38 \\
26.21 \\
26.07 \\
25.35 \\
25.56\end{array}$ & $\begin{array}{l}76-12-08 \\
77-03-08 \\
77-06-15 \\
77-09-13 \\
77-12-06\end{array}$ & $\begin{array}{l}2900 \\
3200 \\
3250 \\
3100 \\
3200\end{array}$ & $\begin{array}{l}7.5 \\
7.6 \\
7.5 \\
7.3 \\
7.4\end{array}$ & $\begin{array}{l}11.5 \\
12.5 \\
12.5 \\
12.0 \\
10.5\end{array}$ & $\begin{array}{l}1200 \\
1100 \\
1200 \\
1100 \\
1100\end{array}$ & $\begin{array}{l}760 \\
730 \\
760 \\
710 \\
730\end{array}$ & $\begin{array}{l}320 \\
320 \\
330 \\
320 \\
310\end{array}$ \\
\hline $\begin{array}{l}78-04-09 \\
78-06-20\end{array}$ & $\begin{array}{l}26.56 \\
26.04\end{array}$ & $\begin{array}{l}78-03-09 \\
78-06-20\end{array}$ & $\begin{array}{l}3400 \\
3000\end{array}$ & $\begin{array}{l}7.4 \\
7.6\end{array}$ & $\begin{array}{l}11.0 \\
12.0\end{array}$ & & $\begin{array}{l}780 \\
770\end{array}$ & $\begin{array}{l}330 \\
330\end{array}$ \\
\hline $\begin{array}{c}\text { DATE OF } \\
\text { WATER-LEVEL } \\
\text { MEASUREMENT }\end{array}$ & $\begin{array}{l}\text { WATER } \\
\text { LEVEL } \\
\text { (FEET } \\
\text { BELOW } \\
\text { LAND } \\
\text { SURFACE) }\end{array}$ & $\begin{array}{c}\text { DATE } \\
\text { OF } \\
\text { SAMPLE }\end{array}$ & $\begin{array}{l}\text { FLUO- } \\
\text { RIDE, } \\
\text { DIS- } \\
\text { SOLVED } \\
\text { (MG/L } \\
\text { AS F) }\end{array}$ & $\begin{array}{l}\text { SILICA, } \\
\text { DIS- } \\
\text { SOLVED } \\
\text { (MG/L } \\
\text { AS } \\
\text { SI02) }\end{array}$ & $\begin{array}{l}\text { SOL IDS, } \\
\text { SUM OF } \\
\text { CONSTI- } \\
\text { TUENTS, } \\
\text { DIS-- } \\
\text { SOLVED } \\
\text { (MG/L) }\end{array}$ & $\begin{array}{c}\text { NITRO- } \\
\text { GEN, } \\
\text { NITRATE } \\
\text { DIS- } \\
\text { SOLVED } \\
(\text { MG/L } \\
\text { AS N) }\end{array}$ & $\begin{array}{l}\text { NITRO- } \\
\text { GEN, } \\
\text { NITRITE } \\
\text { DIS- } \\
\text { SOLVED } \\
\text { (MG/L } \\
\text { AS N) }\end{array}$ & $\begin{array}{c}\text { NITRO- } \\
\text { GEN, } \\
\text { NO2+NO3 } \\
\text { DIS- } \\
\text { SOLVED } \\
\text { (MG/L } \\
\text { AS N) }\end{array}$ \\
\hline $\begin{array}{l}74-04-04 \\
74-04-08 \\
74-06-14 \\
74-07-23 \\
74-08-27\end{array}$ & $\begin{array}{l}26.58 \\
26.90 \\
23.78 \\
25.82 \\
25.43\end{array}$ & $\begin{array}{l}74-04-04 \\
74-05-09 \\
74-06-14 \\
74-07-24 \\
74-08-28\end{array}$ & $\begin{array}{r}1.1 \\
1.0 \\
.9 \\
.9 \\
1.0\end{array}$ & $\begin{array}{l}19 \\
19 \\
22 \\
19 \\
19\end{array}$ & $\begin{array}{l}2210 \\
2070 \\
2050 \\
2040 \\
2050\end{array}$ & $\begin{array}{l}.73 \\
1.5 \\
1.7 \\
-- \\
--\end{array}$ & $\begin{array}{l}.02 \\
.01 \\
.01 \\
.- \\
.00\end{array}$ & $\begin{array}{l}.75 \\
1.5 \\
1.7 \\
.88 \\
.83\end{array}$ \\
\hline $\begin{array}{l}74-10-03 \\
74-11-11 \\
74-12-05 \\
75-02-06 \\
75-05-14\end{array}$ & $\begin{array}{l}24.82 \\
25.48 \\
26.00 \\
26.51 \\
25.88\end{array}$ & $\begin{array}{l}74-10-04 \\
74-11-11 \\
74-12-05 \\
75-02-06 \\
75-05-14\end{array}$ & $\begin{array}{r}1.0 \\
.8 \\
1.4 \\
.9 \\
.9\end{array}$ & $\begin{array}{l}19 \\
18 \\
22 \\
19 \\
18\end{array}$ & $\begin{array}{l}2190 \\
2010 \\
2200 \\
2350 \\
2300\end{array}$ & $\begin{array}{l}1.1 \\
.94 \\
1.0 \\
1.0 \\
.74\end{array}$ & $\begin{array}{l}.00 \\
.01 \\
.00 \\
.00 \\
.00\end{array}$ & $\begin{array}{l}1.1 \\
.95 \\
1.0 \\
1.0 \\
.74\end{array}$ \\
\hline $\begin{array}{l}75-08-11 \\
75-12-02 \\
76-03-29 \\
76-06-28 \\
76-09-22\end{array}$ & $\begin{array}{l}25.49 \\
25.08 \\
26.24 \\
25.66 \\
24.71\end{array}$ & $\begin{array}{l}75-08-11 \\
75-12-02 \\
76-03-29 \\
76-06-28 \\
76-09-22\end{array}$ & $\begin{array}{r}1.0 \\
1.1 \\
.9 \\
.9 \\
1.0\end{array}$ & $\begin{array}{l}18 \\
18 \\
18 \\
18 \\
18\end{array}$ & $\begin{array}{l}2310 \\
2340 \\
2230 \\
2130 \\
2350\end{array}$ & $\begin{array}{l}1.4 \\
1.1 \\
1.6 \\
1.7 \\
1.9\end{array}$ & $\begin{array}{l}.01 \\
.01 \\
.00 \\
.00 \\
.00\end{array}$ & $\begin{array}{l}1.4 \\
1.1 \\
1.6 \\
1.7 \\
1.9\end{array}$ \\
\hline $\begin{array}{l}76-12-08 \\
77-03-08 \\
77-06-15 \\
77-09-13 \\
77-12-06\end{array}$ & $\begin{array}{l}25.38 \\
26.21 \\
26.07 \\
25.35 \\
25.56\end{array}$ & $\begin{array}{l}76-12-08 \\
77-03-08 \\
77-06-15 \\
77-09-13 \\
77-12-06\end{array}$ & $\begin{array}{l}.9 \\
.9 \\
1.0 \\
1.0 \\
1.0\end{array}$ & $\begin{array}{l}17 \\
19 \\
18 \\
20 \\
17\end{array}$ & $\begin{array}{l}2340 \\
2270 \\
2350 \\
2300 \\
2460\end{array}$ & $\begin{array}{l}.70 \\
1.8 \\
1.7 \\
2.6 \\
.69\end{array}$ & $\begin{array}{l}.00 \\
.01 \\
.00 \\
.00 \\
.01\end{array}$ & $\begin{array}{l}.70 \\
1.8 \\
1.7 \\
2.6 \\
.70\end{array}$ \\
\hline $\begin{array}{l}78-04-09 \\
78-06-20\end{array}$ & 26.04 & $\begin{array}{l}78-03-09 \\
78-06-20\end{array}$ & $\begin{array}{l}.9 \\
.9\end{array}$ & $\begin{array}{l}16 \\
15\end{array}$ & $\begin{array}{l}2480 \\
2410\end{array}$ & $\begin{array}{l}.60 \\
1.8\end{array}$ & $\begin{array}{l}.01 \\
.03\end{array}$ & $\begin{array}{l}.61 \\
1.8\end{array}$ \\
\hline
\end{tabular}


analyses of water from observation wells--Continued

Depth is $89 \mathrm{ft}$. Interval open to the aquifer is 59 to $89 \mathrm{ft}$ ]

\begin{tabular}{|c|c|c|c|c|c|c|c|c|}
\hline $\begin{array}{l}\text { MAGNE- } \\
\text { SIUM, } \\
\text { DIS- } \\
\text { SOLVED } \\
\text { (MG/L } \\
\text { AS MG) }\end{array}$ & $\begin{array}{l}\text { SODIUM, } \\
\text { DIS- } \\
\text { SOLVED } \\
\text { (MG/L } \\
\text { AS NA) }\end{array}$ & $\begin{array}{l}\text { SODIUM } \\
\text { AD- } \\
\text { SORP- } \\
\text { TION } \\
\text { RATIO }\end{array}$ & $\begin{array}{l}\text { POTAS- } \\
\text { SIUM, } \\
\text { DIS- } \\
\text { SOLVED } \\
\text { (MG/L } \\
\text { AS K) }\end{array}$ & $\begin{array}{c}\text { BICAR- } \\
\text { BONATE } \\
\text { (MG/L } \\
\text { AS } \\
\text { HCO3) }\end{array}$ & $\begin{array}{c}\text { CAR- } \\
\text { BONATE } \\
\text { (MG/L } \\
\text { AS CO3) }\end{array}$ & $\begin{array}{c}\text { ALKA- } \\
\text { LINITY } \\
\text { (MG/L } \\
\text { AS } \\
\text { CAC03) }\end{array}$ & $\begin{array}{l}\text { SULFATE, } \\
\text { DIS- } \\
\text { SOLVED } \\
\text { (MG/L } \\
\text { AS SO4) }\end{array}$ & $\begin{array}{l}\text { CHLO- } \\
\text { RIDE, } \\
\text { DIS- } \\
\text { SOLVED } \\
\text { (MG/L } \\
\text { AS CL) }\end{array}$ \\
\hline $\begin{array}{l}73 \\
73 \\
73 \\
72 \\
71\end{array}$ & $\begin{array}{l}340 \\
290 \\
300 \\
300 \\
300\end{array}$ & $\begin{array}{l}4.6 \\
3.9 \\
4.1 \\
4.2 \\
4.1\end{array}$ & $\begin{array}{l}7.6 \\
7.8 \\
7.3 \\
6.1 \\
9.0\end{array}$ & $\begin{array}{l}474 \\
474 \\
468 \\
455 \\
454\end{array}$ & $\begin{array}{r}0 \\
0 \\
0 \\
-- \\
--\end{array}$ & $\begin{array}{l}389 \\
389 \\
384 \\
373 \\
372\end{array}$ & $\begin{array}{l}1100 \\
1000 \\
1000 \\
1000 \\
1000\end{array}$ & $\begin{array}{l}140 \\
150 \\
130 \\
140 \\
140\end{array}$ \\
\hline $\begin{array}{l}71 \\
71 \\
74 \\
80 \\
73\end{array}$ & $\begin{array}{l}300 \\
300 \\
310 \\
320 \\
320\end{array}$ & $\begin{array}{l}4.0 \\
4.3 \\
4.2 \\
4.2 \\
4.2\end{array}$ & $\begin{array}{l}8.5 \\
5.1 \\
6.7 \\
8.4 \\
6.9\end{array}$ & $\begin{array}{l}458 \\
453 \\
456 \\
473 \\
461\end{array}$ & $\begin{array}{l}-- \\
-- \\
-- \\
-0\end{array}$ & $\begin{array}{l}376 \\
372 \\
374 \\
388 \\
378\end{array}$ & $\begin{array}{l}1100 \\
1000 \\
1100 \\
1200 \\
1200\end{array}$ & $\begin{array}{l}140 \\
140 \\
150 \\
170 \\
140\end{array}$ \\
\hline $\begin{array}{l}85 \\
78 \\
78 \\
78 \\
80\end{array}$ & $\begin{array}{l}310 \\
340 \\
310 \\
310 \\
320\end{array}$ & $\begin{array}{l}4.0 \\
4.5 \\
4.1 \\
4.1 \\
4.1\end{array}$ & $\begin{array}{l}8.1 \\
8.5 \\
8.6 \\
8.0 \\
8.4\end{array}$ & $\begin{array}{l}472 \\
465 \\
506 \\
515 \\
494\end{array}$ & $\begin{array}{l}0 \\
0 \\
0 \\
0 \\
0\end{array}$ & $\begin{array}{l}387 \\
381 \\
415 \\
422 \\
405\end{array}$ & $\begin{array}{l}1200 \\
1200 \\
1100 \\
1000 \\
1200\end{array}$ & $\begin{array}{l}140 \\
150 \\
150 \\
150 \\
150\end{array}$ \\
\hline $\begin{array}{l}85 \\
80 \\
80 \\
80 \\
88\end{array}$ & $\begin{array}{l}320 \\
330 \\
320 \\
340 \\
340\end{array}$ & $\begin{array}{l}4.1 \\
4.3 \\
4.1 \\
4.4 \\
4.4\end{array}$ & $\begin{array}{l}8.2 \\
7.7 \\
8.0 \\
7.8 \\
8.1\end{array}$ & $\begin{array}{l}472 \\
492 \\
480 \\
510 \\
490\end{array}$ & $\begin{array}{l}0 \\
0 \\
0 \\
0 \\
0\end{array}$ & $\begin{array}{l}387 \\
404 \\
394 \\
420 \\
400\end{array}$ & $\begin{array}{l}1200 \\
1100 \\
1200 \\
1100 \\
1300\end{array}$ & $\begin{array}{l}150 \\
160 \\
150 \\
170 \\
150\end{array}$ \\
\hline $\begin{array}{l}88 \\
88\end{array}$ & $\begin{array}{l}340 \\
360\end{array}$ & $\begin{array}{l}4.3 \\
4.5\end{array}$ & $\begin{array}{l}8.3 \\
8.0\end{array}$ & $\begin{array}{l}490 \\
510\end{array}$ & $\begin{array}{l}0 \\
0\end{array}$ & $\begin{array}{l}400 \\
420\end{array}$ & $\begin{array}{l}1300 \\
1200\end{array}$ & $\begin{array}{l}150 \\
150\end{array}$ \\
\hline
\end{tabular}

NITRO- NITRO- PHOS-

\begin{tabular}{|c|c|c|c|c|c|}
\hline $\begin{array}{l}\text { GEN, } \\
\text { MOOIIA, } \\
\text { JIS- } \\
\text { SOLVED } \\
(\because G / L \\
\text { AS N) }\end{array}$ & $\begin{array}{l}\text { GEN, AM- } \\
\text { MONIA + } \\
\text { ORGANIC } \\
\text { DIS. } \\
(M G / L \\
\text { AS N) }\end{array}$ & $\begin{array}{c}\text { PHORUS, } \\
\text { ORTHO, } \\
\text { DIS- } \\
\text { SOLVED } \\
\text { (MG/L } \\
\text { AS P) }\end{array}$ & $\begin{array}{l}\text { IRON, } \\
\text { DIS- } \\
\text { SOLVED } \\
\text { (UG/L } \\
\text { AS FE) }\end{array}$ & $\begin{array}{l}\text { MANGA- } \\
\text { NESE, } \\
\text { DIS- } \\
\text { SOLVED } \\
\text { (UG/L } \\
\text { AS MN) }\end{array}$ & $\begin{array}{c}\text { CARBON } \\
\text { ORGANIC } \\
\text { DIS- } \\
\text { SOLVED } \\
\text { (MG/L } \\
\text { AS C) }\end{array}$ \\
\hline $\begin{array}{l}.04 \\
.08 \\
.08 \\
.- \\
.04\end{array}$ & $\begin{array}{l}.45 \\
.52 \\
.61 \\
. . \\
.49\end{array}$ & $\begin{array}{l}.02 \\
.00 \\
.02 \\
.05 \\
.07\end{array}$ & $\begin{array}{l}120 \\
460 \\
270 \\
300 \\
250\end{array}$ & $\begin{array}{l}2000 \\
1500 \\
1600 \\
1700 \\
1700\end{array}$ & $\begin{array}{l}-- \\
-- \\
-- \\
-\end{array}$ \\
\hline $\begin{array}{l}.02 \\
.09 \\
.04 \\
.02 \\
.00\end{array}$ & $\begin{array}{l}.43 \\
.52 \\
.58 \\
.46 \\
.22\end{array}$ & $\begin{array}{l}.01 \\
.03 \\
.05 \\
.02 \\
.02\end{array}$ & $\begin{array}{l}250 \\
240 \\
190 \\
280 \\
290\end{array}$ & $\begin{array}{l}1600 \\
1900 \\
1400 \\
1500 \\
1700\end{array}$ & $\begin{array}{l}-- \\
=- \\
-- \\
--\end{array}$ \\
\hline $\begin{array}{l}.03 \\
.01 \\
.03 \\
.00 \\
.00\end{array}$ & $\begin{array}{l}.63 \\
.62 \\
.59 \\
.37 \\
.48\end{array}$ & $\begin{array}{l}.04 \\
.04 \\
.01 \\
.05 \\
.03\end{array}$ & $\begin{array}{l}260 \\
210 \\
240 \\
220 \\
270\end{array}$ & $\begin{array}{l}1400 \\
1400 \\
1700 \\
1800 \\
2100\end{array}$ & $\begin{array}{l}-- \\
-- \\
-- \\
6.8 \\
4.6\end{array}$ \\
\hline $\begin{array}{l}.00 \\
.00 \\
.00 \\
.01 \\
.03\end{array}$ & $\begin{array}{l}.60 \\
.90 \\
.41 \\
.60 \\
.41\end{array}$ & $\begin{array}{l}.01 \\
.05 \\
.04 \\
.02 \\
.02\end{array}$ & $\begin{array}{l}-\overline{370} \\
420 \\
290 \\
370\end{array}$ & $\begin{array}{l}2200 \\
2100 \\
1800 \\
1800 \\
2000\end{array}$ & $\begin{array}{l}-- \\
-- \\
-- \\
-- \\
--\end{array}$ \\
\hline $\begin{array}{l}.01 \\
.01\end{array}$ & $\begin{array}{r}.99 \\
.62\end{array}$ & $\begin{array}{l}.01 \\
.03\end{array}$ & $\begin{array}{l}440 \\
340\end{array}$ & $\begin{array}{l}2400 \\
2100\end{array}$ & -- \\
\hline
\end{tabular}


Table 5.--Water-level records and chemical [We11 number 11 on figure 2. Local we11 number is SB00506423CCC1.

\begin{tabular}{|c|c|c|c|c|c|c|c|c|}
\hline $\begin{array}{l}\text { DATE OF } \\
\text { WATER-LEVEL } \\
\text { MEASUREMENT }\end{array}$ & $\begin{array}{l}\text { WATER } \\
\text { LEVEL } \\
\text { (FEET } \\
\text { BELOW } \\
\text { LAND } \\
\text { SURFACE) }\end{array}$ & $\begin{array}{c}\text { DATE } \\
\text { OF } \\
\text { SAMPLE }\end{array}$ & $\begin{array}{l}\text { SPE- } \\
\text { CIFIC } \\
\text { CON- } \\
\text { DUCT- } \\
\text { ANCE } \\
\text { (MICRO- } \\
\text { MHOS) }\end{array}$ & $\begin{array}{c}\text { PH } \\
\text { (UNITS) }\end{array}$ & $\begin{array}{l}\text { TEMPER- } \\
\text { ATURE } \\
\text { (DEG C) }\end{array}$ & $\begin{array}{l}\text { HARD- } \\
\text { NESS } \\
\text { (MG/L } \\
\text { AS } \\
\text { CACO3) }\end{array}$ & $\begin{array}{l}\text { HARD- } \\
\text { NESS, } \\
\text { NONCAR- } \\
\text { BONATE } \\
\text { (MG/L } \\
\text { CACO3) }\end{array}$ & $\begin{array}{c}\text { CALCIUM, } \\
\text { DIS- } \\
\text { SOLVED } \\
\text { (MG/L } \\
\text { AS CA) }\end{array}$ \\
\hline $\begin{array}{l}74-04-03 \\
74-05-08 \\
74-06-13 \\
74-07-23 \\
74-08-27\end{array}$ & $\begin{array}{l}27.33 \\
27.65 \\
26.99 \\
25.60 \\
24.17\end{array}$ & $\begin{array}{l}74-04-03 \\
74-05-08 \\
74-06-13 \\
74-07-24 \\
74-08-27\end{array}$ & $\begin{array}{l}2370 \\
2320 \\
2370 \\
2350 \\
2270\end{array}$ & $\begin{array}{l}7.2 \\
7.3 \\
7.4 \\
7.3 \\
-.\end{array}$ & $\begin{array}{l}11.5 \\
12.5 \\
12.5 \\
13.5 \\
12.5\end{array}$ & $\begin{array}{l}890 \\
870 \\
890 \\
860 \\
850\end{array}$ & $\begin{array}{l}560 \\
540 \\
570 \\
540 \\
540\end{array}$ & $\begin{array}{l}200 \\
190 \\
200 \\
190 \\
190\end{array}$ \\
\hline $\begin{array}{l}74-10-03 \\
74-11-11 \\
74-12-05 \\
75-02-06 \\
75-05-14\end{array}$ & $\begin{array}{l}25.18 \\
26.03 \\
26.45 \\
27.32 \\
28.20\end{array}$ & $\begin{array}{l}74-10-04 \\
74-11-11 \\
74-12-05 \\
75-02-06 \\
75-05-14\end{array}$ & $\begin{array}{l}2310 \\
2300 \\
2500 \\
2450 \\
2900\end{array}$ & $\begin{array}{l}-- \\
7.4 \\
7.0 \\
7.1 \\
7.1\end{array}$ & $\begin{array}{l}12.0 \\
12.0 \\
12.0 \\
11.5 \\
12.5\end{array}$ & $\begin{array}{l}860 \\
850 \\
880 \\
870 \\
910\end{array}$ & $\begin{array}{l}550 \\
530 \\
560 \\
550 \\
600\end{array}$ & $\begin{array}{l}190 \\
200 \\
200 \\
190 \\
200\end{array}$ \\
\hline $\begin{array}{l}75-08-12 \\
75-12-02 \\
76-03-29 \\
76-06-29 \\
76-09-22\end{array}$ & $\begin{array}{l}25.04 \\
25.89 \\
27.39 \\
27.89 \\
24.97\end{array}$ & $\begin{array}{l}75-08-12 \\
75-12-02 \\
76-03-29 \\
76-06-29 \\
76-09-22\end{array}$ & $\begin{array}{l}3100 \\
2700 \\
2200 \\
2500 \\
2200\end{array}$ & $\begin{array}{l}7.2 \\
7.5 \\
7.5 \\
6.5 \\
7.1\end{array}$ & $\begin{array}{l}13.5 \\
13.0 \\
12.0 \\
13.0 \\
12.5\end{array}$ & $\begin{array}{l}910 \\
850 \\
910 \\
890 \\
940\end{array}$ & $\begin{array}{l}590 \\
520 \\
590 \\
560 \\
590\end{array}$ & $\begin{array}{l}200 \\
190 \\
200 \\
190 \\
210\end{array}$ \\
\hline $\begin{array}{l}76-12-08 \\
77-03-08 \\
77-06-15 \\
77-09-13 \\
77-12-06\end{array}$ & $\begin{array}{l}26.54 \\
27.18 \\
26.89 \\
25.47 \\
26.23\end{array}$ & $\begin{array}{l}76-12-08 \\
77-03-08 \\
77-06-15 \\
77-09-13 \\
77-12-06\end{array}$ & $\begin{array}{l}2300 \\
2600 \\
2600 \\
2600 \\
2400\end{array}$ & $\begin{array}{l}7.4 \\
7.6 \\
7.3 \\
7.4 \\
7.3\end{array}$ & $\begin{array}{l}12.0 \\
13.0 \\
13.0 \\
13.0 \\
12.0\end{array}$ & $\begin{array}{l}890 \\
910 \\
940 \\
960 \\
980\end{array}$ & $\begin{array}{l}550 \\
570 \\
590 \\
600 \\
590\end{array}$ & $\begin{array}{l}200 \\
200 \\
210 \\
220 \\
210\end{array}$ \\
\hline $\begin{array}{l}78-03-09 \\
78-06-20\end{array}$ & $\begin{array}{l}27.43 \\
27.99\end{array}$ & $\begin{array}{l}78-03-09 \\
78-06-20\end{array}$ & $\begin{array}{l}2600 \\
2500\end{array}$ & $\begin{array}{l}7.3 \\
7.7\end{array}$ & $\begin{array}{l}13.0 \\
13.5\end{array}$ & $\begin{array}{r}1000 \\
960\end{array}$ & $\begin{array}{l}630 \\
600\end{array}$ & $\begin{array}{l}220 \\
220\end{array}$ \\
\hline $\begin{array}{l}\text { DATE OF } \\
\text { WATER-LEVEL } \\
\text { MEASUREMENT }\end{array}$ & $\begin{array}{l}\text { WATER } \\
\text { LEVEL } \\
\text { (FEET } \\
\text { BELOW } \\
\text { LAND } \\
\text { SURFACE) }\end{array}$ & $\begin{array}{c}\text { DATE } \\
\text { OF } \\
\text { SAMPLE }\end{array}$ & $\begin{array}{l}\text { FLUO- } \\
\text { RIDE, } \\
\text { DIS- } \\
\text { SOLVED } \\
\text { (MG/L } \\
\text { AS F) }\end{array}$ & $\begin{array}{l}\text { SILICA, } \\
\text { DIS- } \\
\text { SOLVEO } \\
\text { (MG/L } \\
\text { AS } \\
\text { SI02) }\end{array}$ & $\begin{array}{l}\text { SOLIDS, } \\
\text { SUM OF } \\
\text { CONSTI- } \\
\text { TUENTS, } \\
\text { OIS- } \\
\text { SOLVED } \\
\text { (MG/L) }\end{array}$ & $\begin{array}{c}\text { NITRO- } \\
\text { GEN, } \\
\text { NITRATE } \\
\text { DIS- } \\
\text { SOLVED } \\
\text { (MG/L } \\
\text { AS N) }\end{array}$ & $\begin{array}{l}\text { NITRO- } \\
\text { GEN, } \\
\text { NITRITE } \\
\text { DIS- } \\
\text { SOLVED } \\
\text { (MG/L } \\
\text { AS N) }\end{array}$ & $\begin{array}{c}\text { NITRO- } \\
\text { GEN, } \\
\text { NO2+NO3 } \\
\text { DIS- } \\
\text { SOLVED } \\
\text { (MG/L } \\
\text { AS N) }\end{array}$ \\
\hline $\begin{array}{l}74-04-03 \\
74-05-08 \\
74-06-13 \\
74-07-23 \\
74-08-27\end{array}$ & $\begin{array}{l}27.33 \\
27.65 \\
26.99 \\
25.60 \\
24.17\end{array}$ & $\begin{array}{l}74-04-03 \\
74-05-08 \\
74-06-13 \\
74-07-24 \\
74-08-27\end{array}$ & $\begin{array}{l}1.9 \\
1.9 \\
3.4 \\
1.8 \\
1.8\end{array}$ & $\begin{array}{l}24 \\
24 \\
29 \\
25 \\
25\end{array}$ & $\begin{array}{l}1710 \\
1690 \\
1680 \\
1660 \\
1620\end{array}$ & $\begin{array}{l}7.8 \\
8.3 \\
7.5 \\
-- \\
--\end{array}$ & $\begin{array}{l}.12 \\
.02 \\
.01 \\
. \overline{.01}\end{array}$ & $\begin{array}{l}7.9 \\
8.3 \\
7.5 \\
8.1 \\
8.2\end{array}$ \\
\hline $\begin{array}{l}74-10-03 \\
74-11-11 \\
74-12-05 \\
75-02-06 \\
75-05-14\end{array}$ & $\begin{array}{l}25.18 \\
26.03 \\
26.45 \\
27.32 \\
28.20\end{array}$ & $\begin{array}{l}74-10-04 \\
74-11-11 \\
74-12-05 \\
75-02-06 \\
75-05-14\end{array}$ & $\begin{array}{l}1.7 \\
1.4 \\
1.6 \\
1.7 \\
1.8\end{array}$ & $\begin{array}{l}25 \\
25 \\
24 \\
25 \\
23\end{array}$ & $\begin{array}{l}1650 \\
1670 \\
1640 \\
1640 \\
1690\end{array}$ & $\begin{array}{l}7.0 \\
7.9 \\
9.0 \\
8.7\end{array}$ & $\begin{array}{l}.01 \\
.00 \\
.00 \\
.00 \\
.00\end{array}$ & $\begin{array}{l}8.6 \\
7.0 \\
7.9 \\
9.0 \\
8.7\end{array}$ \\
\hline $\begin{array}{l}75-08-12 \\
75-12-02 \\
76-03-29 \\
76-06-29 \\
76-09-22\end{array}$ & $\begin{array}{l}25.04 \\
25.89 \\
27.39 \\
27.89 \\
24.97\end{array}$ & $\begin{array}{l}75-08-12 \\
75-12-02 \\
76-03-29 \\
76-06-29 \\
76-09-22\end{array}$ & $\begin{array}{l}1.7 \\
1.8 \\
1.8 \\
1.8 \\
1.8\end{array}$ & $\begin{array}{l}23 \\
23 \\
23 \\
22 \\
23\end{array}$ & $\begin{array}{l}1760 \\
1640 \\
1690 \\
1750 \\
1840\end{array}$ & $\begin{array}{l}7.9 \\
7.2 \\
7.4 \\
7.7 \\
9.2\end{array}$ & $\begin{array}{l}.01 \\
.01 \\
.00 \\
.00 \\
.01\end{array}$ & $\begin{array}{l}7.9 \\
7.2 \\
7.4 \\
7.7 \\
9.2\end{array}$ \\
\hline $\begin{array}{l}76-12-08 \\
77-03-08 \\
77-06-15 \\
77-09-13 \\
77-12-06\end{array}$ & $\begin{array}{l}26.54 \\
27.18 \\
26.89 \\
25.47 \\
26.23\end{array}$ & $\begin{array}{l}76-12-08 \\
77-03-08 \\
77-06-15 \\
77-09-13 \\
77-12-06\end{array}$ & $\begin{array}{l}1.8 \\
1.8 \\
1.8 \\
1.8 \\
1.8\end{array}$ & $\begin{array}{l}23 \\
24 \\
23 \\
25 \\
25\end{array}$ & $\begin{array}{l}1780 \\
1730 \\
1780 \\
1860 \\
1780\end{array}$ & $\begin{array}{c}11 \\
7.6 \\
7.5 \\
7.9 \\
12\end{array}$ & $\begin{array}{l}.00 \\
.00 \\
.00 \\
.01 \\
.05\end{array}$ & $\begin{array}{c}11 \\
7.6 \\
7.5 \\
7.9 \\
12\end{array}$ \\
\hline $\begin{array}{l}78-03-09 \\
78-06-20\end{array}$ & $\begin{array}{l}27.43 \\
27.99\end{array}$ & $\begin{array}{l}78-03-09 \\
78-06-20\end{array}$ & $\begin{array}{l}1.7 \\
1.7\end{array}$ & $\begin{array}{l}22 \\
20\end{array}$ & $\begin{array}{l}1790 \\
1800\end{array}$ & $\begin{array}{l}9.7 \\
7.7\end{array}$ & $\begin{array}{l}.17 \\
.16\end{array}$ & $\begin{array}{l}9.9 \\
7.9\end{array}$ \\
\hline
\end{tabular}


analyses of water from observation wells--Continued

Depth is $78 \mathrm{ft}$. Interval open to the aquifer is 48 to $78 \mathrm{ft}$ ]

\begin{tabular}{|c|c|c|c|c|c|c|c|c|}
\hline $\begin{array}{l}\text { MAGNE- } \\
\text { SIUM, } \\
\text { DIS- } \\
\text { SOLVED } \\
\text { (MG/L } \\
\text { AS MG) }\end{array}$ & $\begin{array}{l}\text { SODIUM, } \\
\text { DIS- } \\
\text { SOLVED } \\
\text { (MG/L } \\
\text { AS NA) }\end{array}$ & $\begin{array}{c}\text { SODIUM } \\
\text { AD- } \\
\text { SORP- } \\
\text { TION } \\
\text { RATIO }\end{array}$ & $\begin{array}{c}\text { POTAS- } \\
\text { SIUM, } \\
\text { DIS- } \\
\text { SOLVED } \\
\text { (MG/L } \\
\text { AS K) }\end{array}$ & $\begin{array}{c}\text { BICAR- } \\
\text { BONATE } \\
\text { (MG/L } \\
\text { AS } \\
\text { HCO3) }\end{array}$ & $\begin{array}{c}\text { CAR- } \\
\text { BONATE } \\
(M G / L \\
\text { AS CO3) }\end{array}$ & $\begin{array}{l}\text { ALKA- } \\
\text { LINITY } \\
\text { (MG/L } \\
\text { AS } \\
\text { CACO3) }\end{array}$ & $\begin{array}{l}\text { SULFATE, } \\
\text { DIS- } \\
\text { SOLVED } \\
\text { (MG/L } \\
\text { AS SO4) }\end{array}$ & $\begin{array}{l}\text { CHLO- } \\
\text { RIDE, } \\
\text { DIS- } \\
\text { SOLVED } \\
\text { (MG/L } \\
\text { AS CL) }\end{array}$ \\
\hline $\begin{array}{l}96 \\
95 \\
96 \\
94 \\
92\end{array}$ & $\begin{array}{l}230 \\
230 \\
220 \\
230 \\
220\end{array}$ & $\begin{array}{l}3.3 \\
3.4 \\
3.2 \\
3.4 \\
3.3\end{array}$ & $\begin{array}{l}6.7 \\
6.2 \\
6.4 \\
6.3 \\
7.6\end{array}$ & $\begin{array}{l}404 \\
394 \\
390 \\
394 \\
387\end{array}$ & $\begin{array}{r}0 \\
0 \\
0 \\
-- \\
--\end{array}$ & $\begin{array}{l}331 \\
323 \\
320 \\
323 \\
317\end{array}$ & $\begin{array}{l}790 \\
790 \\
770 \\
750 \\
740\end{array}$ & $\begin{array}{l}130 \\
120 \\
130 \\
130 \\
120\end{array}$ \\
\hline $\begin{array}{l}94 \\
84 \\
92 \\
95 \\
99\end{array}$ & $\begin{array}{l}210 \\
220 \\
210 \\
210 \\
220\end{array}$ & $\begin{array}{l}3.1 \\
3.3 \\
3.1 \\
3.1 \\
3.2\end{array}$ & $\begin{array}{l}7.0 \\
4.4 \\
6.1 \\
6.7 \\
5.7\end{array}$ & $\begin{array}{l}383 \\
379 \\
384 \\
390 \\
377\end{array}$ & $\begin{array}{l}-- \\
-- \\
-- \\
0\end{array}$ & $\begin{array}{l}314 \\
311 \\
315 \\
320 \\
309\end{array}$ & $\begin{array}{l}780 \\
800 \\
760 \\
750 \\
780\end{array}$ & $\begin{array}{l}120 \\
120 \\
120 \\
130 \\
140\end{array}$ \\
\hline $\begin{array}{r}99 \\
90 \\
100 \\
100 \\
100\end{array}$ & $\begin{array}{l}220 \\
220 \\
210 \\
240 \\
230\end{array}$ & $\begin{array}{l}3.2 \\
3.3 \\
3.0 \\
3.5 \\
3.3\end{array}$ & $\begin{array}{l}7.1 \\
6.9 \\
7.0 \\
6.6 \\
6.7\end{array}$ & $\begin{array}{l}386 \\
401 \\
395 \\
402 \\
419\end{array}$ & $\begin{array}{l}0 \\
0 \\
0 \\
0 \\
0\end{array}$ & $\begin{array}{l}317 \\
329 \\
324 \\
330 \\
344\end{array}$ & $\begin{array}{l}830 \\
760 \\
780 \\
810 \\
880\end{array}$ & $\begin{array}{l}150 \\
120 \\
140 \\
150 \\
140\end{array}$ \\
\hline $\begin{array}{r}96 \\
100 \\
100 \\
100 \\
110\end{array}$ & $\begin{array}{l}230 \\
230 \\
230 \\
240 \\
220\end{array}$ & $\begin{array}{l}3.3 \\
3.3 \\
3.3 \\
3.4 \\
3.1\end{array}$ & $\begin{array}{l}6.6 \\
6.4 \\
6.5 \\
6.5 \\
6.6\end{array}$ & $\begin{array}{l}421 \\
412 \\
420 \\
440 \\
470\end{array}$ & $\begin{array}{l}0 \\
0 \\
0 \\
0 \\
0\end{array}$ & $\begin{array}{l}345 \\
338 \\
344 \\
360 \\
390\end{array}$ & $\begin{array}{l}830 \\
830 \\
820 \\
860 \\
800\end{array}$ & $\begin{array}{r}140 \\
96 \\
150 \\
150 \\
120\end{array}$ \\
\hline $\begin{array}{l}110 \\
100\end{array}$ & $\begin{array}{l}210 \\
240\end{array}$ & $\begin{array}{l}2.9 \\
3.4\end{array}$ & $\begin{array}{l}7.4 \\
6.6\end{array}$ & $\begin{array}{l}460 \\
440\end{array}$ & $\begin{array}{l}0 \\
0\end{array}$ & $\begin{array}{l}380 \\
360\end{array}$ & $\begin{array}{l}810 \\
790\end{array}$ & $\begin{array}{l}140 \\
170\end{array}$ \\
\hline
\end{tabular}

NITRO- NITRO- PHOS-

GEN, GEN,AM- PHORUS,

$\begin{array}{cccccc}\text { AMMONIA } & \text { MONIA + } & \text { ORTHO, } & \text { IRON, } & \text { NESE, } & \text { ORGANIC } \\ \text { DIS- } & \text { ORGANIC } & \text { DIS- } & \text { DIS- } & \text { DIS- } & \text { DIS- } \\ \text { SOLVED } & \text { DIS. } & \text { SOLVED } & \text { SOLVED } & \text { SOLVED } & \text { SOLVED } \\ \text { (MG/L } & \text { (MG/L } & \text { (MG/L } & \text { (UG/L } & \text { (UG/L } & \text { (MG/L }\end{array}$

$\begin{array}{llllll}(M G / L & (M G / L & (M G / L & (U G / L & (U G / L & (M G / L \\ A S N) & A S N) & A S P) & A S F E) & A S M N) & A S C)\end{array}$

$\begin{array}{lccccc}.10 & .66 & .03 & 50 & 30 & -- \\ .07 & .51 & .04 & 30 & 0 & -- \\ .03 & .69 & .02 & 10 & 0 & -- \\ .03 & .56 & .05 & 40 & 0 & -- \\ .03 & .56 & 30 & 0 & -- \\ .01 & .31 & .02 & 80 & 0 & -- \\ .01 & .56 & .03 & 20 & 0 & -- \\ .05 & .37 & .04 & 10 & 10 & -- \\ .01 & .46 & .09 & 30 & 20 & -- \\ .00 & .34 & .03 & 30 & 10 & -- \\ .00 & .38 & .03 & 20 & 0 & -- \\ .00 & .73 & .05 & 20 & 5 & -- \\ .02 & .80 & .02 & 10 & 0 & -- \\ .00 & .29 & .04 & 30 & 0 & 4.3 \\ .00 & .40 & .03 & 30 & 10 & 4.9 \\ .00 & .96 & .02 & 70 & 0 & -- \\ .01 & 1.1 & .09 & 50 & 0 & -- \\ .00 & .39 & .04 & 20 & 0 & -- \\ .01 & .56 & .03 & 30 & 10 & -- \\ .00 & .39 & .02 & 40 & 4 & -- \\ .00 & .84 & .02 & 10 & 10 & -- \\ .01 & 1.2 & .02 & 80 & 0 & --\end{array}$


Table 5.--Water-level records and chemical [We11 number 13 on figure 2. Local we11 number is SB00506423DCB1.

\begin{tabular}{|c|c|c|c|c|c|c|c|c|}
\hline $\begin{array}{l}\text { DATE OF } \\
\text { WATER-LEVEL } \\
\text { MEASUREMENT }\end{array}$ & $\begin{array}{l}\text { WATER } \\
\text { LEVEL } \\
\text { (FEET } \\
\text { BELOW } \\
\text { LAND } \\
\text { SURFACE) }\end{array}$ & $\begin{array}{l}\text { DATE } \\
\text { OF } \\
\text { SAMPLE }\end{array}$ & $\begin{array}{l}\text { SPE- } \\
\text { CIFIC } \\
\text { CON- } \\
\text { DUCT- } \\
\text { ANCE } \\
\text { (MICRO- } \\
\text { MHOS) }\end{array}$ & $\begin{array}{c}\text { PH } \\
\text { (UNITS) }\end{array}$ & $\begin{array}{l}\text { TEMPER- } \\
\text { ATURE } \\
\text { (DEG C) }\end{array}$ & $\begin{array}{l}\text { HARD- } \\
\text { NESS } \\
(M G / L \\
\text { AS } \\
\text { CACO3) }\end{array}$ & $\begin{array}{l}\text { HARD- } \\
\text { NESS, } \\
\text { NONCAR- } \\
\text { BONATE } \\
\text { (MG/L } \\
\text { CACO3) }\end{array}$ & $\begin{array}{l}\text { CALCIUM, } \\
\text { DIS- } \\
\text { SOLVED } \\
\text { (MG/L } \\
\text { AS CA) }\end{array}$ \\
\hline $\begin{array}{l}73-12-14 \\
74-04-03 \\
74-05-08 \\
74-06-13 \\
74-07-23\end{array}$ & $\begin{array}{l}36.80 \\
37.58 \\
38.34 \\
38.48 \\
38.52\end{array}$ & $\begin{array}{l}74-04-03 \\
74-05-08 \\
74-06-13 \\
74-07-23\end{array}$ & $\begin{array}{l}---. \\
2290 \\
2390 \\
2380 \\
2390\end{array}$ & $\begin{array}{l}-.- \\
7.4 \\
7.4 \\
7.5 \\
7.4\end{array}$ & $\begin{array}{l}---. \\
12.5 \\
13.5 \\
14.0 \\
14.5\end{array}$ & $\begin{array}{l}--- \\
930 \\
930 \\
900 \\
930\end{array}$ & $\begin{array}{l}--- \\
660 \\
650 \\
630 \\
660\end{array}$ & $\begin{array}{l}-20 \\
220 \\
210 \\
220\end{array}$ \\
\hline $\begin{array}{l}74-03-27 \\
74-10-03 \\
74-11-11 \\
74-12-05 \\
75-02-06\end{array}$ & $\begin{array}{l}38.54 \\
38.13 \\
37.78 \\
37.86 \\
37.49\end{array}$ & $\begin{array}{l}74-08-27 \\
74-10-03 \\
74-11-11 \\
74-12-05 \\
75-02-06\end{array}$ & $\begin{array}{l}2390 \\
2390 \\
2400 \\
2400 \\
2500\end{array}$ & $\begin{array}{l}-- \\
-- \\
7.2 \\
7.1 \\
7.2\end{array}$ & $\begin{array}{l}13.5 \\
13.5 \\
13.0 \\
13.0 \\
13.0\end{array}$ & $\begin{array}{l}920 \\
920 \\
850 \\
920 \\
910\end{array}$ & $\begin{array}{l}640 \\
630 \\
580 \\
640 \\
630\end{array}$ & $\begin{array}{l}220 \\
220 \\
220 \\
210 \\
210\end{array}$ \\
\hline $\begin{array}{l}75-05-14 \\
75-08-11 \\
75-12-01 \\
76-03-29 \\
76-06-29\end{array}$ & $\begin{array}{l}38.15 \\
38.77 \\
38.54 \\
38.23 \\
38.85\end{array}$ & $\begin{array}{l}75-05-14 \\
75-08-11 \\
75-12-01 \\
76-03-29 \\
76-06-29\end{array}$ & $\begin{array}{l}2900 \\
3000 \\
2650 \\
2250 \\
2300\end{array}$ & $\begin{array}{l}7.3 \\
7.3 \\
7.1 \\
7.6 \\
7.1\end{array}$ & $\begin{array}{l}13.5 \\
14.5 \\
13.0 \\
12.0 \\
13.5\end{array}$ & $\begin{array}{l}900 \\
900 \\
920 \\
910 \\
900\end{array}$ & $\begin{array}{l}630 \\
640 \\
650 \\
640 \\
630\end{array}$ & $\begin{array}{l}220 \\
210 \\
220 \\
220 \\
210\end{array}$ \\
\hline $\begin{array}{l}76-09-22 \\
75-12-08 \\
77-03-08 \\
77-05-15 \\
77-09-13\end{array}$ & $\begin{array}{l}39.41 \\
37.64 \\
38.07 \\
39.09 \\
38.40\end{array}$ & $\begin{array}{l}76-09-22 \\
76-12-08 \\
77-03-08 \\
77-06-15 \\
77-09-12\end{array}$ & $\begin{array}{l}2200 \\
2200 \\
2300 \\
2370 \\
2000\end{array}$ & $\begin{array}{l}7.2 \\
7.3 \\
7.5 \\
7.4 \\
7.3\end{array}$ & $\begin{array}{l}13.5 \\
12.5 \\
13.0 \\
13.5 \\
13.5\end{array}$ & $\begin{array}{l}920 \\
890 \\
890 \\
890 \\
910\end{array}$ & $\begin{array}{l}660 \\
630 \\
620 \\
620 \\
640\end{array}$ & $\begin{array}{l}220 \\
210 \\
210 \\
210 \\
210\end{array}$ \\
\hline $\begin{array}{l}77-12-06 \\
78-03-09 \\
78-06-20\end{array}$ & $\begin{array}{l}37.71 \\
37.85 \\
38.90\end{array}$ & $\begin{array}{l}77-12-06 \\
78-03-09 \\
78-06-20\end{array}$ & $\begin{array}{l}2200 \\
2300 \\
2300\end{array}$ & $\begin{array}{l}7.2 \\
7.2 \\
7.3\end{array}$ & $\begin{array}{l}12.5 \\
13.0 \\
13.5\end{array}$ & $\begin{array}{l}940 \\
940 \\
910\end{array}$ & $\begin{array}{l}670 \\
660 \\
630\end{array}$ & $\begin{array}{l}220 \\
220 \\
200\end{array}$ \\
\hline $\begin{array}{l}\text { DATE OF } \\
\text { WATER-LEVEL } \\
\text { MEASUREMENT }\end{array}$ & $\begin{array}{l}\text { WATER } \\
\text { LEVEL } \\
\text { (FEET } \\
\text { BELOW } \\
\text { LAND } \\
\text { SURFACE) }\end{array}$ & $\begin{array}{l}\text { DATE } \\
\text { OF } \\
\text { SAMPLE }\end{array}$ & $\begin{array}{l}\text { FLUO- } \\
\text { RIDE, } \\
\text { DIS- } \\
\text { SOLVED } \\
\text { (MG/L } \\
\text { AS F) }\end{array}$ & $\begin{array}{l}\text { SILICA, } \\
\text { DIS- } \\
\text { SOLVED } \\
\text { (MG/L } \\
\text { AS } \\
\text { SI02) }\end{array}$ & $\begin{array}{l}\text { SOLIDS, } \\
\text { SUM OF } \\
\text { CONSTI- } \\
\text { TUENTS, } \\
\text { DIS- } \\
\text { SOLVED } \\
\text { (MG/L) }\end{array}$ & $\begin{array}{l}\text { NITRO- } \\
\text { GEN, } \\
\text { NITRATE } \\
\text { DIS- } \\
\text { SOLVED } \\
(M G / L \\
\text { AS N) }\end{array}$ & $\begin{array}{l}\text { NITRO- } \\
\text { GEN, } \\
\text { NITRITE } \\
\text { DIS- } \\
\text { SOLVED } \\
(M G / L \\
\text { AS N) }\end{array}$ & $\begin{array}{l}\text { NITRO- } \\
\text { GEN, } \\
\text { NO2+NO3 } \\
\text { DIS- } \\
\text { SOLVED } \\
\text { (MG/L } \\
\text { AS N) }\end{array}$ \\
\hline $\begin{array}{l}73-12-14 \\
74-04-03 \\
74-05-08 \\
74-06-13 \\
74-07-23\end{array}$ & $\begin{array}{l}36.80 \\
37.58 \\
38.34 \\
38.48 \\
38.52\end{array}$ & $\begin{array}{l}74-04-03 \\
74-05-08 \\
74-06-13 \\
74-07-23\end{array}$ & $\begin{array}{l}--- \\
1.2 \\
1.2 \\
1.1 \\
1.2\end{array}$ & $\begin{array}{l}-- \\
20 \\
21 \\
25 \\
21\end{array}$ & $\begin{array}{l}---- \\
1690 \\
1760 \\
1680 \\
1740\end{array}$ & $\begin{array}{l}4.7 \\
5.0 \\
4.6 \\
--\end{array}$ & $\begin{array}{l}.- \\
.01 \\
.00 \\
.00 \\
--\end{array}$ & $\begin{array}{l}-.- \\
4.7 \\
5.0 \\
4.6 \\
5.0\end{array}$ \\
\hline $\begin{array}{l}74-08-27 \\
74-10-03 \\
74-11-11 \\
74-12-05 \\
75-02-06\end{array}$ & $\begin{array}{l}38.54 \\
38.13 \\
37.78 \\
37.86 \\
37.49\end{array}$ & $\begin{array}{l}74-08-27 \\
74-10-03 \\
74-11-11 \\
74-12-05 \\
75-02-06\end{array}$ & $\begin{array}{l}1.3 \\
1.2 \\
1.0 \\
1.2 \\
1.1\end{array}$ & $\begin{array}{l}21 \\
22 \\
21 \\
21 \\
21\end{array}$ & $\begin{array}{l}1690 \\
1730 \\
1660 \\
1700 \\
1760\end{array}$ & $\begin{array}{l}-- \\
-- \\
5.2 \\
4.8 \\
5.0\end{array}$ & $\begin{array}{l}.01 \\
.01 \\
.00 \\
.00 \\
.00\end{array}$ & $\begin{array}{l}4.1 \\
5.0 \\
5.2 \\
4.8 \\
5.0\end{array}$ \\
\hline $\begin{array}{l}75-05-14 \\
75-08-11 \\
75-12-01 \\
76-03-29 \\
76-06-29\end{array}$ & $\begin{array}{l}38.15 \\
38.77 \\
38.54 \\
38.23 \\
38.85\end{array}$ & $\begin{array}{l}75-05-14 \\
75-08-11 \\
75-12-01 \\
76-03-29 \\
76-06-29\end{array}$ & $\begin{array}{l}1.1 \\
1.0 \\
1.1 \\
1.1 \\
1.2\end{array}$ & $\begin{array}{l}20 \\
20 \\
20 \\
20 \\
19\end{array}$ & $\begin{array}{l}1690 \\
1750 \\
1740 \\
1670 \\
1770\end{array}$ & $\begin{array}{r}4.9 \\
4.9 \\
4.7 \\
-.9\end{array}$ & $\begin{array}{l}.00 \\
.01 \\
.01 \\
.- \\
.00\end{array}$ & $\begin{array}{l}4.9 \\
4.9 \\
4.7 \\
5.1 \\
4.9\end{array}$ \\
\hline $\begin{array}{l}76-09-22 \\
76-12-08 \\
77-03-08 \\
77-06-15 \\
77-09-13\end{array}$ & $\begin{array}{l}39.41 \\
37.64 \\
38.07 \\
39.09 \\
38.40\end{array}$ & $\begin{array}{l}76-09-22 \\
76-12-08 \\
77-03-08 \\
77-06-15 \\
77-09-12\end{array}$ & $\begin{array}{l}1.1 \\
1.1 \\
1.2 \\
1.2 \\
1.2\end{array}$ & $\begin{array}{l}20 \\
20 \\
21 \\
20 \\
22\end{array}$ & $\begin{array}{l}1750 \\
1700 \\
1700 \\
1690 \\
1710\end{array}$ & $\begin{array}{l}4.9 \\
4.8 \\
4.7 \\
5.3 \\
5.0\end{array}$ & $\begin{array}{l}.01 \\
.00 \\
.01 \\
.00 \\
.00\end{array}$ & $\begin{array}{l}4.9 \\
4.8 \\
4.7 \\
5.3 \\
5.0\end{array}$ \\
\hline $\begin{array}{l}77-12-06 \\
78-03-09 \\
78-06-20\end{array}$ & $\begin{array}{l}37.71 \\
37.85 \\
38.90\end{array}$ & $\begin{array}{l}77-12-06 \\
78-03-09 \\
78-06-20\end{array}$ & $\begin{array}{l}1.2 \\
1.3 \\
1.6\end{array}$ & $\begin{array}{l}21 \\
19 \\
18\end{array}$ & $\begin{array}{l}1740 \\
1720 \\
1710\end{array}$ & $\begin{array}{l}6.0 \\
5.5 \\
7.8\end{array}$ & $\begin{array}{l}.01 \\
.00 \\
.02\end{array}$ & $\begin{array}{l}6.0 \\
5.5 \\
7.8\end{array}$ \\
\hline
\end{tabular}


analyses of water from observation wells--Continued

Depth is $122 \mathrm{ft}$. Interval open to the aquifer is 118 to $122 \mathrm{ft}$ ]

\begin{tabular}{|c|c|c|c|c|c|c|c|}
\hline $\begin{array}{l}\text { MAGNE- } \\
\text { SIUM, } \\
\text { DIS- } \\
\text { SOLVED } \\
\text { (MG/L } \\
\text { AS MG) }\end{array}$ & $\begin{array}{l}\text { SODIUH, } \\
\text { DIS- } \\
\text { SOLVED } \\
\text { (MG/L } \\
\text { AS NA) }\end{array}$ & $\begin{array}{l}\text { SODIUM } \\
\text { AD- } \\
\text { SORP- } \\
\text { TION } \\
\text { RATIO }\end{array}$ & $\begin{array}{c}\text { POTAS- } \\
\text { SIUM, } \\
\text { DIS- } \\
\text { SOLVED } \\
\text { (MG/L } \\
\text { AS K) }\end{array}$ & $\begin{array}{c}\text { BICAR- } \\
\text { BONATE } \\
\text { (MG/L } \\
\text { AS } \\
\text { HCO3) }\end{array}$ & $\begin{array}{c}\text { CAR- } \\
\text { BONATE } \\
(M G / L \\
\text { AS CO3) }\end{array}$ & $\begin{array}{l}\text { ALKA- } \\
\text { LINITY } \\
\text { (MG/L } \\
\text { AS } \\
\text { CACO3) }\end{array}$ & $\begin{array}{c}\text { SULFATE, } \\
\text { DIS- } \\
\text { SOLVED } \\
\text { (MG/L } \\
\text { AS SO4) }\end{array}$ \\
\hline $\begin{array}{l}-- \\
92 \\
92 \\
92 \\
93\end{array}$ & $\begin{array}{l}--- \\
210 \\
210 \\
210 \\
220\end{array}$ & $\begin{array}{l}--- \\
3.0 \\
3.0 \\
3.0 \\
3.1\end{array}$ & $\begin{array}{l}-.- \\
5.2 \\
5.6 \\
5.3 \\
5.4\end{array}$ & $\begin{array}{l}--- \\
329 \\
334 \\
332 \\
338\end{array}$ & $\begin{array}{r}-- \\
0 \\
0 \\
0 \\
--\end{array}$ & $\begin{array}{l}270 \\
274 \\
272 \\
277\end{array}$ & $\begin{array}{l}--- \\
820 \\
870 \\
810 \\
840\end{array}$ \\
\hline $\begin{array}{l}91 \\
90 \\
74 \\
95 \\
93\end{array}$ & $\begin{array}{l}210 \\
210 \\
230 \\
210 \\
210\end{array}$ & $\begin{array}{l}3.0 \\
3.0 \\
3.4 \\
3.0 \\
3.0\end{array}$ & $\begin{array}{l}6.7 \\
6.1 \\
3.5 \\
5.1 \\
5.6\end{array}$ & $\begin{array}{l}344 \\
349 \\
333 \\
340 \\
335\end{array}$ & $\begin{array}{l}-- \\
-- \\
-- \\
--\end{array}$ & $\begin{array}{l}282 \\
286 \\
273 \\
279 \\
275\end{array}$ & $\begin{array}{l}810 \\
840 \\
780 \\
830 \\
870\end{array}$ \\
\hline $\begin{array}{l}85 \\
92 \\
90 \\
88 \\
90\end{array}$ & $\begin{array}{l}220 \\
220 \\
230 \\
200 \\
230\end{array}$ & $\begin{array}{l}3.2 \\
3.2 \\
3.3 \\
2.9 \\
3.3\end{array}$ & $\begin{array}{l}5.1 \\
6.0 \\
5.7 \\
5.7 \\
5.5\end{array}$ & $\begin{array}{l}327 \\
327 \\
331 \\
337 \\
328\end{array}$ & $\begin{array}{l}0 \\
0 \\
0 \\
0 \\
0\end{array}$ & $\begin{array}{l}268 \\
268 \\
271 \\
276 \\
269\end{array}$ & $\begin{array}{l}810 \\
870 \\
850 \\
810 \\
880\end{array}$ \\
\hline $\begin{array}{l}90 \\
89 \\
88 \\
89 \\
93\end{array}$ & $\begin{array}{l}220 \\
220 \\
220 \\
220 \\
220\end{array}$ & $\begin{array}{l}3.2 \\
3.2 \\
3.2 \\
3.2 \\
3.2\end{array}$ & $\begin{array}{l}5.5 \\
5.5 \\
5.5 \\
5.3 \\
5.1\end{array}$ & $\begin{array}{l}323 \\
319 \\
324 \\
330 \\
330\end{array}$ & $\begin{array}{l}0 \\
0 \\
0 \\
0 \\
0\end{array}$ & $\begin{array}{l}265 \\
262 \\
266 \\
270 \\
270\end{array}$ & $\begin{array}{l}870 \\
830 \\
820 \\
820 \\
820\end{array}$ \\
\hline $\begin{array}{l}94 \\
94 \\
99\end{array}$ & $\begin{array}{l}220 \\
210 \\
220\end{array}$ & $\begin{array}{l}3.1 \\
3.0 \\
3.2\end{array}$ & $\begin{array}{l}5.3 \\
5.8 \\
6.0\end{array}$ & $\begin{array}{l}330 \\
340 \\
340\end{array}$ & $\begin{array}{l}0 \\
0 \\
0\end{array}$ & $\begin{array}{l}270 \\
280 \\
280\end{array}$ & $\begin{array}{l}840 \\
830 \\
840\end{array}$ \\
\hline
\end{tabular}

NITRO- NITRO- PHOS-

$\begin{array}{llll}\text { GEN, GEN,AM- } & \text { PHORUS, } & \text { MANGA- } & \text { CARBON, } \\ & \text { MMMONIA MONIA + ORTHO, IRON, NESE, ORGANIC }\end{array}$

DIS- ORGANIC DIS- DIS- DIS- DIS-

SOLVED DIS. SOLVED SOLVED SOLVED SOLVED

$\begin{array}{llllll}(M G / L & (M G / L & (M G / L & (U G / L & (U G / L & (M G / L \\ A S N) & A S N) & A S P) & A S F E) & A S M N) & A S C)\end{array}$

$\begin{array}{lllrrr}.01 & .- & .- & -- & -- & -- \\ .02 & .47 & .02 & 40 & 0 & -- \\ .02 & .54 & .01 & 20 & 20 & -- \\ -- & -- & .02 & 0 & 10 & -- \\ .03 & .45 & .06 & 20 & 0 & -- \\ .04 & .45 & .03 & 80 & 0 & -- \\ .04 & .61 & .01 & 30 & 30 & -- \\ .08 & .40 & .03 & 10 & 0 & -- \\ .01 & .43 & .01 & 10 & 0 & -- \\ .00 & .25 & .04 & 20 & 5 & -- \\ .00 & .47 & .03 & 10 & 0 & -- \\ .00 & .73 & .04 & 60 & 5 & -- \\ -- & -- & .01 & 10 & 10 & -- \\ .00 & .32 & .04 & 80 & 0 & 4.5 \\ .00 & .26 & .05 & 10 & 10 & 4.7 \\ .00 & .77 & .01 & 30 & 0 & -- \\ .00 & .40 & .04 & 70 & 20 & -- \\ .00 & .47 & .03 & 90 & 0 & -- \\ .01 & .40 & .04 & 70 & 0 & -- \\ .01 & .48 & .01 & 60 & 0 & -- \\ .00 & .55 & .01 & 120 & 0 & -- \\ .00 & .92 & .02 & 160 & 10 & --\end{array}$


Table 5.--Water-level records and chemical [We11 number 14 on figure 2. Local wel1 number is SB00506423DCB2.

\begin{tabular}{|c|c|c|c|c|c|c|c|c|}
\hline $\begin{array}{l}\text { DATE OF } \\
\text { WATER-LEVEL } \\
\text { MEASUREMENT }\end{array}$ & $\begin{array}{l}\text { WATER } \\
\text { LEVEL } \\
\text { (FEET } \\
\text { BELOW } \\
\text { LAND } \\
\text { SURFACE) }\end{array}$ & $\begin{array}{c}\text { DATE } \\
\text { OF } \\
\text { SAMPLE }\end{array}$ & $\begin{array}{l}\text { SPE- } \\
\text { CIFIC } \\
\text { CON- } \\
\text { DUCT- } \\
\text { ANCE } \\
\text { (MICRO- } \\
\text { MHOS) }\end{array}$ & $\begin{array}{c}\mathrm{PH} \\
\text { (UNITS) }\end{array}$ & $\begin{array}{l}\text { TEMPER- } \\
\text { ATURE } \\
\text { (DEG C) }\end{array}$ & $\begin{array}{l}\text { HARD- } \\
\text { NESS } \\
\text { (MG/L } \\
\text { AS } \\
\text { CACO3) }\end{array}$ & $\begin{array}{l}\text { HARD- } \\
\text { NESS, } \\
\text { NONCAR- } \\
\text { BONATE } \\
\text { (MG/L } \\
\text { CACO3) }\end{array}$ & $\begin{array}{l}\text { CALCIUM, } \\
\text { DIS- } \\
\text { SOLVED } \\
\text { (MG/L } \\
\text { AS CA) }\end{array}$ \\
\hline $\begin{array}{l}73-12-15 \\
74-04-03 \\
74-05-08 \\
74-05-13 \\
74-07-23\end{array}$ & $\begin{array}{l}36.30 \\
36.69 \\
36.97 \\
37.11 \\
36.82\end{array}$ & $\begin{array}{l}74-04-03 \\
74-05-08 \\
74-06-13 \\
74-07-23\end{array}$ & $\begin{array}{l}-\overrightarrow{2540} \\
2520 \\
2520 \\
2540\end{array}$ & $\begin{array}{l}-.-5 \\
7.4 \\
7.4 \\
7.4 \\
7.2\end{array}$ & $\begin{array}{l}---. \\
12.5 \\
13.5 \\
13.5 \\
14.5\end{array}$ & $\begin{array}{r}960 \\
1000 \\
960 \\
1000\end{array}$ & $\begin{array}{l}--- \\
640 \\
680 \\
640 \\
680\end{array}$ & $\begin{array}{l}--- \\
220 \\
220 \\
220 \\
220\end{array}$ \\
\hline $\begin{array}{l}74-08-27 \\
74-10-03 \\
74-11-11 \\
74-12-05 \\
75-02-06\end{array}$ & $\begin{array}{l}36.50 \\
36.19 \\
36.26 \\
36.41 \\
36.86\end{array}$ & $\begin{array}{l}74-08-27 \\
74-10-03 \\
74-11-11 \\
74-12-05 \\
75-02-06\end{array}$ & $\begin{array}{l}2530 \\
2510 \\
2400 \\
2500 \\
2600\end{array}$ & $\begin{array}{l}-- \\
-. \\
7.3 \\
7.1 \\
7.2\end{array}$ & $\begin{array}{l}13.0 \\
14.0 \\
12.5 \\
13.0 \\
12.5\end{array}$ & $\begin{array}{l}980 \\
990 \\
840 \\
910 \\
940\end{array}$ & $\begin{array}{l}660 \\
670 \\
530 \\
590 \\
610\end{array}$ & $\begin{array}{l}210 \\
230 \\
210 \\
200 \\
210\end{array}$ \\
\hline $\begin{array}{l}75-05-14 \\
75-08-11 \\
75-12-01 \\
76-03-29 \\
76-06-29\end{array}$ & $\begin{array}{l}37.32 \\
37.01 \\
36.14 \\
37.12 \\
37.52\end{array}$ & $\begin{array}{l}75-05-14 \\
75-08-11 \\
75-12-01 \\
76-03-29 \\
76-06-29\end{array}$ & $\begin{array}{r}2900 \\
3100 \\
2650 \\
=- \\
2500\end{array}$ & $\begin{array}{l}7.4 \\
7.2 \\
7.2 \\
-.0 \\
7.1\end{array}$ & $\begin{array}{r}13.0 \\
14.0 \\
12.5 \\
-.- \\
13.0\end{array}$ & $\begin{array}{l}940 \\
890 \\
880 \\
880 \\
910\end{array}$ & $\begin{array}{l}620 \\
570 \\
560 \\
560 \\
600\end{array}$ & $\begin{array}{l}210 \\
200 \\
200 \\
200 \\
200\end{array}$ \\
\hline $\begin{array}{l}76-09-22 \\
76-12-08 \\
77-03-08 \\
77-06-15 \\
77-09-13\end{array}$ & $\begin{array}{l}36.48 \\
36.38 \\
37.06 \\
37.65 \\
37.17\end{array}$ & $\begin{array}{l}76-09-22 \\
76-12-08 \\
77-03-08 \\
77-06-15 \\
77-09-13\end{array}$ & $\begin{array}{l}2200 \\
2200 \\
2400 \\
2450 \\
2400\end{array}$ & $\begin{array}{l}7.2 \\
7.3 \\
7.3 \\
7.3 \\
7.3\end{array}$ & $\begin{array}{l}13.0 \\
12.5 \\
13.0 \\
13.0 \\
13.0\end{array}$ & $\begin{array}{l}900 \\
910 \\
890 \\
940 \\
940\end{array}$ & $\begin{array}{l}580 \\
590 \\
570 \\
620 \\
600\end{array}$ & $\begin{array}{l}200 \\
200 \\
200 \\
210 \\
210\end{array}$ \\
\hline $\begin{array}{l}77-12-06 \\
78-03-09 \\
78-06-20\end{array}$ & $\begin{array}{l}35.93 \\
36.98 \\
37.33\end{array}$ & $\begin{array}{l}77-12-06 \\
78-03-09 \\
78-06-20\end{array}$ & $\begin{array}{l}2400 \\
2400 \\
2400\end{array}$ & $\begin{array}{l}7.2 \\
7.2 \\
7.4\end{array}$ & $\begin{array}{l}12.0 \\
12.5 \\
13.0\end{array}$ & $\begin{array}{l}940 \\
980 \\
980\end{array}$ & $\begin{array}{l}590 \\
810 \\
640\end{array}$ & $\begin{array}{l}210 \\
210 \\
210\end{array}$ \\
\hline $\begin{array}{l}\text { DATE OF } \\
\text { WATER-LEVEL } \\
\text { MEASUREMENT }\end{array}$ & $\begin{array}{l}\text { WATER } \\
\text { LEVEL } \\
\text { (FEET } \\
\text { BELOW } \\
\text { LAND } \\
\text { SURFACE) }\end{array}$ & $\begin{array}{c}\text { DATE } \\
\text { OF } \\
\text { SAMPLE }\end{array}$ & $\begin{array}{l}\text { FLUO- } \\
\text { RIDE, } \\
\text { DIS- } \\
\text { SOLVED } \\
\text { (MG/L } \\
\text { AS F) }\end{array}$ & $\begin{array}{l}\text { SILICA, } \\
\text { DIS- } \\
\text { SOLVED } \\
\text { (MG/L } \\
\text { AS } \\
\text { SI02) }\end{array}$ & $\begin{array}{l}\text { SOLIDS, } \\
\text { SUM OF } \\
\text { CONSTI- } \\
\text { TUENTS, } \\
\text { DIS- } \\
\text { SOLVED } \\
(M G / L)\end{array}$ & $\begin{array}{l}\text { NITRO- } \\
\text { GEN, } \\
\text { NITRATE } \\
\text { DIS- } \\
\text { SOLVED } \\
\text { (MG/L } \\
\text { AS N) }\end{array}$ & $\begin{array}{c}\text { NITRO- } \\
\text { GEN, } \\
\text { NITRITE } \\
\text { DIS- } \\
\text { SOLVED } \\
\text { (MG/L } \\
\text { AS N) }\end{array}$ & $\begin{array}{l}\text { NITRO- } \\
\text { GEN, } \\
\text { NO2+NO3 } \\
\text { DIS- } \\
\text { SOLVED } \\
\text { (MG/L } \\
\text { AS N) }\end{array}$ \\
\hline $\begin{array}{l}73-12-15 \\
74-04-03 \\
74-05-08 \\
74-05-13 \\
74-07-23\end{array}$ & $\begin{array}{l}36.30 \\
36.69 \\
36.97 \\
37.11 \\
36.82\end{array}$ & $\begin{array}{l}74-04-03 \\
74-05-08 \\
74-05-13 \\
74-07-23\end{array}$ & $\begin{array}{l}--5 \\
1.7 \\
1.4 \\
1.5 \\
1.6\end{array}$ & $\begin{array}{l}-- \\
24 \\
25 \\
28\end{array}$ & $\begin{array}{l}1820 \\
1880 \\
1810 \\
1860\end{array}$ & $\begin{array}{l}8.8 \\
8.8 \\
8.8 \\
--\end{array}$ & $\begin{array}{l}.0 \\
.00 \\
.00 \\
.01 \\
.-\end{array}$ & $\begin{array}{l}8 . \overline{-} \\
8.8 \\
8.8 \\
8.2\end{array}$ \\
\hline $\begin{array}{l}74-08-27 \\
74-10-03 \\
74-11-11 \\
74-12-05 \\
75-02-06\end{array}$ & $\begin{array}{l}36.50 \\
36.19 \\
36.26 \\
36.41 \\
36.86\end{array}$ & $\begin{array}{l}74-08-27 \\
74-10-03 \\
74-11-11 \\
74-12-05 \\
75-02-06\end{array}$ & $\begin{array}{l}1.5 \\
1.5 \\
1.4 \\
1.5 \\
1.4\end{array}$ & $\begin{array}{l}24 \\
25 \\
24 \\
23 \\
24\end{array}$ & $\begin{array}{l}1910 \\
1840 \\
1700 \\
1770 \\
1830\end{array}$ & $\begin{array}{c}10^{--} \\
9.0 \\
9.6\end{array}$ & $\begin{array}{l}.01 \\
.01 \\
.00 \\
.00 \\
.00\end{array}$ & $\begin{array}{c}8.2 \\
8.6 \\
10 \\
9.0 \\
9.6\end{array}$ \\
\hline $\begin{array}{l}75-05-14 \\
75-08-11 \\
75-12-01 \\
76-03-29 \\
76-06-29\end{array}$ & $\begin{array}{l}37.32 \\
37.01 \\
36.14 \\
37.12 \\
37.52\end{array}$ & $\begin{array}{l}75-05-14 \\
75-08-11 \\
75-12-01 \\
76-03-29 \\
76-05-29\end{array}$ & $\begin{array}{l}1.6 \\
1.4 \\
1.5 \\
1.4 \\
1.6\end{array}$ & $\begin{array}{l}23 \\
23 \\
23 \\
23 \\
22\end{array}$ & $\begin{array}{l}1780 \\
1770 \\
1740 \\
1650 \\
1820\end{array}$ & $\begin{array}{c}10 \\
8.9 \\
9.8 \\
11 \\
9.6\end{array}$ & $\begin{array}{l}.00 \\
.01 \\
.01 \\
.00 \\
.00\end{array}$ & $\begin{array}{c}10 \\
8.9 \\
9.8 \\
11 \\
9.6\end{array}$ \\
\hline $\begin{array}{l}76-09-22 \\
76-12-08 \\
77-03-08 \\
77-05-15 \\
77-09-13\end{array}$ & $\begin{array}{l}36.48 \\
36.38 \\
37.06 \\
37.65 \\
37.17\end{array}$ & $\begin{array}{l}76-09-22 \\
76-12-08 \\
77-03-08 \\
77-06-15 \\
77-09-13\end{array}$ & $\begin{array}{l}1.6 \\
1.5 \\
1.6 \\
1.6 \\
1.6\end{array}$ & $\begin{array}{l}23 \\
22 \\
24 \\
22 \\
25\end{array}$ & $\begin{array}{l}1830 \\
1800 \\
1760 \\
1780 \\
1800\end{array}$ & $\begin{array}{r}10 \\
13 \\
9.5 \\
9.1 \\
9.9\end{array}$ & $\begin{array}{l}.01 \\
.00 \\
.01 \\
.00 \\
.00\end{array}$ & $\begin{array}{l}10 \\
13 \\
9.5 \\
9.1 \\
9.9\end{array}$ \\
\hline $\begin{array}{l}77-12-06 \\
78-03-09 \\
78-06-20\end{array}$ & $\begin{array}{l}35.93 \\
36.98 \\
37.33\end{array}$ & $\begin{array}{l}77-12-06 \\
78-03-09 \\
78-06-20\end{array}$ & $\begin{array}{l}1.6 \\
1.6 \\
1.5\end{array}$ & $\begin{array}{l}25 \\
22 \\
19\end{array}$ & $\begin{array}{l}1800 \\
1690 \\
1820\end{array}$ & $\begin{array}{l}11 \\
10 \\
9.0\end{array}$ & $\begin{array}{l}.01 \\
.02 \\
.01\end{array}$ & $\begin{array}{l}11 \\
10 \\
9.0\end{array}$ \\
\hline
\end{tabular}


analyses of water from observation wells--Continued

\begin{tabular}{|c|c|c|c|c|c|c|c|c|}
\hline $\begin{array}{l}\text { MAGNE- } \\
\text { SIUM, } \\
\text { DIS- } \\
\text { SOLVED } \\
\text { (MG/L } \\
\text { AS MG) }\end{array}$ & $\begin{array}{l}\text { SODIUM, } \\
\text { DIS- } \\
\text { SOLVED } \\
\text { (MG/L } \\
\text { AS NA) }\end{array}$ & $\begin{array}{l}\text { SODIUM } \\
\text { AD- } \\
\text { SORP- } \\
\text { TION } \\
\text { RATIO }\end{array}$ & $\begin{array}{l}\text { POTAS- } \\
\text { SIUM, } \\
\text { DIS- } \\
\text { SOLVED } \\
\text { (MG/L } \\
\text { AS K) }\end{array}$ & $\begin{array}{c}\text { BICAR- } \\
\text { BONATE } \\
\text { (MG/L } \\
\text { AS } \\
\text { HCO3) }\end{array}$ & $\begin{array}{c}\text { CAR- } \\
\text { BONATE } \\
\text { (MG/L } \\
\text { AS CO3) }\end{array}$ & $\begin{array}{l}\text { ALKA- } \\
\text { LINITY } \\
\text { (MG/L } \\
\text { AS } \\
\text { CAC03) }\end{array}$ & $\begin{array}{c}\text { SULFATE, } \\
\text { DIS- } \\
\text { SOLVED } \\
\text { (MG/L } \\
\text { AS SO4) }\end{array}$ & $\begin{array}{l}\text { CHLO- } \\
\text { RIDE, } \\
\text { DIS- } \\
\text { SOLVED } \\
\text { (MG/L } \\
\text { AS CL) }\end{array}$ \\
\hline $\begin{array}{l}-100 \\
110 \\
100 \\
110\end{array}$ & $\begin{array}{l}-2- \\
230 \\
220 \\
230 \\
240\end{array}$ & $\begin{array}{l}3 .-- \\
3.0 \\
3.2 \\
3.3\end{array}$ & $\begin{array}{l}7.0 \\
7.4 \\
6.6 \\
6.5\end{array}$ & $\begin{array}{l}--- \\
395 \\
391 \\
386 \\
388\end{array}$ & $\begin{array}{r}-- \\
0 \\
0 \\
0 \\
--\end{array}$ & $\begin{array}{l}-1- \\
324 \\
321 \\
317 \\
318\end{array}$ & $\begin{array}{l}--- \\
860 \\
910 \\
850 \\
880\end{array}$ & $\begin{array}{l}170 \\
150 \\
140 \\
150\end{array}$ \\
\hline $\begin{array}{r}110 \\
100 \\
77 \\
100 \\
100\end{array}$ & $\begin{array}{l}240 \\
230 \\
250 \\
240 \\
230\end{array}$ & $\begin{array}{l}3.3 \\
3.2 \\
3.8 \\
3.5 \\
3.3\end{array}$ & $\begin{array}{l}9.0 \\
7.8 \\
4.7 \\
6.3 \\
6.9\end{array}$ & $\begin{array}{l}383 \\
385 \\
382 \\
387 \\
394\end{array}$ & $\begin{array}{l}-- \\
-- \\
-- \\
--\end{array}$ & $\begin{array}{l}314 \\
316 \\
313 \\
317 \\
323\end{array}$ & $\begin{array}{l}940 \\
880 \\
770 \\
830 \\
870\end{array}$ & $\begin{array}{l}150 \\
140 \\
130 \\
140 \\
150\end{array}$ \\
\hline $\begin{array}{r}100 \\
95 \\
92 \\
92 \\
100\end{array}$ & $\begin{array}{l}240 \\
230 \\
240 \\
220 \\
250\end{array}$ & $\begin{array}{l}3.4 \\
3.4 \\
3.5 \\
3.2 \\
3.6\end{array}$ & $\begin{array}{l}6.7 \\
7.5 \\
7.3 \\
7.6 \\
7.2\end{array}$ & $\begin{array}{l}391 \\
385 \\
390 \\
389 \\
383\end{array}$ & $\begin{array}{r}0 \\
0 \\
0 \\
-- \\
0\end{array}$ & $\begin{array}{l}321 \\
316 \\
320 \\
319 \\
314\end{array}$ & $\begin{array}{l}810 \\
840 \\
810 \\
730 \\
860\end{array}$ & $\begin{array}{l}150 \\
140 \\
130 \\
140 \\
150\end{array}$ \\
\hline $\begin{array}{r}98 \\
100 \\
96 \\
100 \\
100\end{array}$ & $\begin{array}{l}240 \\
230 \\
240 \\
230 \\
240\end{array}$ & $\begin{array}{l}3.5 \\
3.3 \\
3.5 \\
3.3 \\
3.4\end{array}$ & $\begin{array}{l}7.2 \\
7.1 \\
6.9 \\
6.9 \\
6.6\end{array}$ & $\begin{array}{l}388 \\
392 \\
395 \\
390 \\
410\end{array}$ & $\begin{array}{l}0 \\
0 \\
0 \\
0 \\
0\end{array}$ & $\begin{array}{l}318 \\
322 \\
324 \\
320 \\
340\end{array}$ & $\begin{array}{l}880 \\
850 \\
800 \\
830 \\
820\end{array}$ & $\begin{array}{l}140 \\
140 \\
150 \\
150 \\
150\end{array}$ \\
\hline $\begin{array}{l}100 \\
110 \\
110\end{array}$ & $\begin{array}{l}240 \\
230 \\
240\end{array}$ & $\begin{array}{l}3.4 \\
3.2 \\
3.3\end{array}$ & $\begin{array}{l}7.2 \\
7.5 \\
7.1\end{array}$ & $\begin{array}{l}420 \\
210 \\
410\end{array}$ & $\begin{array}{l}0 \\
0 \\
0\end{array}$ & $\begin{array}{l}340 \\
170 \\
340\end{array}$ & $\begin{array}{l}820 \\
810 \\
840\end{array}$ & $\begin{array}{l}140 \\
150 \\
150\end{array}$ \\
\hline
\end{tabular}

NITRO- NITRO- PHOS-

$\begin{array}{cccccc}\text { GEN, } & \text { GEN,AM- } & \text { PHORUS, } & & \text { MANGA- } & \text { CARBON, } \\ \text { AMMONIA } & \text { MONIA + } & \text { ORTHO, } & \text { IRON, } & \text { NESE, } & \text { ORGANIC } \\ \text { OIS- } & \text { ORGANIC } & \text { DIS- } & \text { DIS- } & \text { DIS- } & \text { DIS- } \\ \text { SOLVED } & \text { DIS. } & \text { SOLVED } & \text { SOLVED } & \text { SOLVED } & \text { SOLVED } \\ (M G / L & \text { (MG/L } & \text { (MG/L } & \text { (UG/L } & \text { (UG/L } & \text { (MG/L } \\ \text { AS N) } & \text { AS N) } & \text { AS P) } & \text { AS FE) } & \text { AS MN) } & \text { AS C) }\end{array}$

$\begin{array}{lllrrr}.- & -- & -- & -- & -- & -- \\ .02 & .39 & .04 & 280 & 20 & -- \\ .02 & .58 & .03 & 10 & 20 & -- \\ -- & .76 & .02 & 30 & 0 & -- \\ .03 & -- & .08 & 20 & 0 & -- \\ .04 & .62 & .08 & 400 & 0 & -- \\ .02 & .41 & .05 & 30 & 10 & -- \\ .03 & .62 & .04 & 20 & 0 & -- \\ .01 & .43 & .05 & 10 & 10 & -- \\ .00 & .04 & .03 & 10 & 10 & -- \\ .00 & .47 & .06 & 20 & 10 & -- \\ .01 & .78 & .04 & 10 & 10 & -- \\ .02 & .65 & .03 & 40 & 0 & -- \\ .00 & .55 & .06 & 60 & 10 & -- \\ .00 & .46 & .04 & 60 & 10 & 3.4 \\ .00 & .65 & .03 & 50 & 0 & -- \\ .00 & .48 & .06 & 100 & 10 & -- \\ .00 & .52 & .04 & 150 & 0 & -- \\ .01 & .75 & .05 & 110 & 10 & -- \\ .00 & .31 & .03 & 150 & 8 & -- \\ .00 & .70 & .02 & 110 & 0 & -- \\ .01 & 1.6 & .04 & 80 & 10 & --\end{array}$


Table 5.--Water-level records and chemical

[We11 number 15 on figure 2. Local we11 number is SB00506423DCB3.

\begin{tabular}{|c|c|c|c|c|c|c|c|c|}
\hline $\begin{array}{c}\text { DATE OF } \\
\text { AATER-LEVEL } \\
\text { \&EASUREMENT }\end{array}$ & $\begin{array}{l}\text { WATER } \\
\text { LEVEL } \\
\text { (FEET } \\
\text { BELOW } \\
\text { LAND } \\
\text { SURFACE) }\end{array}$ & $\begin{array}{c}\text { DATE } \\
\text { OF } \\
\text { SAMPLE }\end{array}$ & $\begin{array}{l}\text { SPE- } \\
\text { CIFIC } \\
\text { CON- } \\
\text { DUCT- } \\
\text { ANCE } \\
\text { (MICRO- } \\
\text { MHOS) }\end{array}$ & $\begin{array}{c}\text { PH } \\
\text { (UNITS) }\end{array}$ & $\begin{array}{l}\text { TEMPER- } \\
\text { ATURE } \\
\text { (DEG C) }\end{array}$ & $\begin{array}{l}\text { HARD- } \\
\text { NESS } \\
\text { (MG/L } \\
\text { AS } \\
\text { CAC03) }\end{array}$ & $\begin{array}{l}\text { HARD- } \\
\text { NESS, } \\
\text { NONCAR- } \\
\text { BONATE } \\
\text { (MG/L } \\
\text { CACO3) }\end{array}$ & $\begin{array}{l}\text { CALCIUM } \\
\text { DIS- } \\
\text { SOLVED } \\
\text { (MG/L } \\
\text { AS CA) }\end{array}$ \\
\hline \begin{tabular}{l}
$74-04-03$ \\
\hdashline $74-06-13$ \\
$74-07-23$ \\
$74-08-27$
\end{tabular} & \begin{tabular}{l}
36.16 \\
\hdashline 36.43 \\
36.32 \\
35.89
\end{tabular} & $\begin{array}{l}74-04-03 \\
74-05-08 \\
74-06-13 \\
74-07-23 \\
74-08-27\end{array}$ & $\begin{array}{l}2290 \\
2290 \\
2320 \\
2330 \\
2370\end{array}$ & $\begin{array}{r}7.3 \\
7.4 \\
7.4 \\
7.3 \\
-. .\end{array}$ & $\begin{array}{l}13.0 \\
13.5 \\
13.5 \\
14.0 \\
13.0\end{array}$ & $\begin{array}{l}900 \\
900 \\
870 \\
900 \\
910\end{array}$ & $\begin{array}{l}580 \\
570 \\
540 \\
570 \\
580\end{array}$ & $\begin{array}{l}220 \\
220 \\
210 \\
220 \\
220\end{array}$ \\
\hline $\begin{array}{l}74-10-03 \\
74-11-11 \\
74-12-05 \\
75-02-06 \\
75-05-14\end{array}$ & $\begin{array}{l}35.53 \\
35.57 \\
35.75 \\
36.18 \\
36.74\end{array}$ & $\begin{array}{l}74-10-03 \\
74-11-11 \\
74-12-05 \\
75-02-06 \\
75-05-14\end{array}$ & $\begin{array}{l}2400 \\
2400 \\
2400 \\
2500 \\
2800\end{array}$ & $\begin{array}{l}-- \\
7.4 \\
6.9 \\
7.1 \\
7.2\end{array}$ & $\begin{array}{l}13.0 \\
12.5 \\
13.0 \\
12.5 \\
13.0\end{array}$ & $\begin{array}{l}940 \\
890 \\
940 \\
920 \\
900\end{array}$ & $\begin{array}{l}610 \\
560 \\
590 \\
570 \\
520\end{array}$ & $\begin{array}{l}230 \\
230 \\
230 \\
220 \\
220\end{array}$ \\
\hline $\begin{array}{l}75-08-11 \\
75-12-01 \\
76-03-29 \\
76-06-29 \\
76-09-22\end{array}$ & $\begin{array}{l}36.36 \\
35.54 \\
36.54 \\
37.13 \\
36.70\end{array}$ & $\begin{array}{l}75-08-11 \\
75-12-01 \\
76-03-29 \\
76-06-29 \\
76-09-22\end{array}$ & $\begin{array}{l}3000 \\
2650 \\
2150 \\
2400 \\
2100\end{array}$ & $\begin{array}{l}7.1 \\
7.0 \\
7.5 \\
7.1 \\
7.1\end{array}$ & $\begin{array}{l}14.0 \\
12.5 \\
12.0 \\
13.0 \\
13.0\end{array}$ & $\begin{array}{l}910 \\
920 \\
900 \\
880 \\
920\end{array}$ & $\begin{array}{l}530 \\
520 \\
520 \\
520 \\
530\end{array}$ & $\begin{array}{l}220 \\
230 \\
220 \\
210 \\
220\end{array}$ \\
\hline $\begin{array}{l}76-12-08 \\
77-03-08 \\
77-06-15 \\
77-09-13 \\
77-12-06\end{array}$ & $\begin{array}{l}35.76 \\
36.41 \\
37.70 \\
36.84 \\
36.16\end{array}$ & $\begin{array}{l}76-12-08 \\
77-03-08 \\
77-06-15 \\
77-09-12 \\
77-12-06\end{array}$ & $\begin{array}{l}2100 \\
2200 \\
2500 \\
2300 \\
2200\end{array}$ & $\begin{array}{l}6.8 \\
7.2 \\
6.9 \\
7.1 \\
6.9\end{array}$ & $\begin{array}{l}12.5 \\
13.0 \\
13.5 \\
13.0 \\
12.0\end{array}$ & $\begin{array}{l}910 \\
900 \\
920 \\
970 \\
930\end{array}$ & $\begin{array}{l}550 \\
500 \\
550 \\
560 \\
560\end{array}$ & $\begin{array}{l}220 \\
220 \\
220 \\
230 \\
220\end{array}$ \\
\hline $\begin{array}{l}78-03-09 \\
78-06-20\end{array}$ & $\begin{array}{l}36.35 \\
36.68\end{array}$ & $\begin{array}{l}78-03-08 \\
78-06-20\end{array}$ & $\begin{array}{l}2400 \\
2300\end{array}$ & $\begin{array}{l}6.8 \\
7.0\end{array}$ & $\begin{array}{l}13.0 \\
13.0\end{array}$ & $\begin{array}{l}950 \\
890\end{array}$ & $\begin{array}{l}890 \\
510\end{array}$ & $\begin{array}{l}230 \\
200\end{array}$ \\
\hline $\begin{array}{l}\text { DATE OF } \\
\text { WATER-LEVEL } \\
\text { MEASUREMENT }\end{array}$ & $\begin{array}{l}\text { WATER } \\
\text { LEVEL } \\
\text { (FEET } \\
\text { BELOW } \\
\text { LAND } \\
\text { SURFACE) }\end{array}$ & $\begin{array}{c}\text { DATE } \\
\text { OF } \\
\text { SAMPLE }\end{array}$ & $\begin{array}{l}\text { FLUO- } \\
\text { RIDE, } \\
\text { DIS- } \\
\text { SOLVED } \\
\text { (MG/L } \\
\text { AS F) }\end{array}$ & $\begin{array}{l}\text { SILICA, } \\
\text { DIS- } \\
\text { SOLVED } \\
\text { (MG/L } \\
\text { AS } \\
\text { SI02) }\end{array}$ & $\begin{array}{l}\text { SOLIOS, } \\
\text { SUM OF } \\
\text { CONSTI- } \\
\text { TUENTS, } \\
\text { DIS- } \\
\text { SOLVED } \\
\text { (MG/L) }\end{array}$ & $\begin{array}{c}\text { NITRO- } \\
\text { GEN, } \\
\text { NITRATE } \\
\text { DIS- } \\
\text { SOLVED } \\
\text { (MG/L } \\
\text { AS N) }\end{array}$ & $\begin{array}{c}\text { NITRO- } \\
\text { GEN, } \\
\text { NITRITE } \\
\text { DIS- } \\
\text { SOLVED } \\
\text { (MG/L } \\
\text { AS N) }\end{array}$ & $\begin{array}{c}\text { NITRO- } \\
\text { GEN, } \\
\text { NO2+NO3 } \\
\text { DIS- } \\
\text { SOLVED } \\
\text { (MG/L } \\
\text { AS N) }\end{array}$ \\
\hline $\begin{array}{l}74-04-03 \\
-74-06-13 \\
74-07-23 \\
74-08-27\end{array}$ & $\begin{array}{l}36.16 \\
-36.43 \\
36.32 \\
35.89\end{array}$ & $\begin{array}{l}74-04-03 \\
74-05-08 \\
74-06-13 \\
74-07-23 \\
74-08-27\end{array}$ & $\begin{array}{l}1.8 \\
2.2 \\
2.6 \\
2.0 \\
2.0\end{array}$ & $\begin{array}{l}26 \\
26 \\
30 \\
26 \\
26\end{array}$ & $\begin{array}{l}1740 \\
1710 \\
1650 \\
1730 \\
1740\end{array}$ & $\begin{array}{l}8.0 \\
8.6 \\
8.8 \\
-\overline{7}\end{array}$ & $\begin{array}{l}.00 \\
.00 \\
.00 \\
.- \\
.01\end{array}$ & $\begin{array}{l}8.0 \\
8.6 \\
8.8 \\
9.8 \\
8.7\end{array}$ \\
\hline $\begin{array}{l}74-10-03 \\
74-11-11 \\
74-12-05 \\
75-02-06 \\
75-05-14\end{array}$ & $\begin{array}{l}35.53 \\
35.57 \\
35.75 \\
36.18 \\
36.74\end{array}$ & $\begin{array}{l}74-10-03 \\
74-11-11 \\
74-12-05 \\
75-02-06 \\
75-05-14\end{array}$ & $\begin{array}{l}1.9 \\
2.0 \\
2.0 \\
1.9 \\
2.0\end{array}$ & $\begin{array}{l}26 \\
26 \\
25 \\
26 \\
25\end{array}$ & $\begin{array}{l}1790 \\
1760 \\
1740 \\
1800 \\
1700\end{array}$ & $\begin{array}{l}11 \\
9.7 \\
10 \\
13\end{array}$ & $\begin{array}{l}.01 \\
.00 \\
.00 \\
.01 \\
.00\end{array}$ & $\begin{array}{l}9.7 \\
11 \\
9.7 \\
10 \\
13\end{array}$ \\
\hline $\begin{array}{l}75-08-11 \\
75-12-01 \\
76-03-29 \\
76-06-29 \\
76-09-22\end{array}$ & $\begin{array}{l}36.36 \\
35.64 \\
36.54 \\
37.13 \\
36.70\end{array}$ & $\begin{array}{l}75-08-11 \\
75-12-01 \\
76-03-29 \\
76-06-29 \\
76-09-22\end{array}$ & $\begin{array}{l}1.7 \\
1.5 \\
1.5 \\
1.5 \\
1.7\end{array}$ & $\begin{array}{l}26 \\
26 \\
27 \\
26 \\
26\end{array}$ & $\begin{array}{l}1710 \\
1800 \\
1670 \\
1760 \\
1800\end{array}$ & $\begin{array}{l}9.8 \\
10 \\
10 \\
8.8 \\
7.7\end{array}$ & $\begin{array}{l}.01 \\
.01 \\
.00 \\
.00 \\
.01\end{array}$ & $\begin{array}{l}9.8 \\
10 \\
10 \\
8.8 \\
7.7\end{array}$ \\
\hline $\begin{array}{l}76-12-08 \\
77-03-08 \\
77-06-15 \\
77-09-13 \\
77-12-06\end{array}$ & $\begin{array}{l}35.76 \\
36.41 \\
37.70 \\
36.84 \\
36.16\end{array}$ & $\begin{array}{l}76-12-08 \\
77-03-08 \\
77-06-15 \\
77-09-12 \\
77-12-06\end{array}$ & $\begin{array}{l}1.2 \\
1.4 \\
1.5 \\
1.5 \\
1.7\end{array}$ & $\begin{array}{l}29 \\
29 \\
28 \\
31 \\
29\end{array}$ & $\begin{array}{l}1750 \\
1730 \\
1740 \\
1770 \\
1750\end{array}$ & $\begin{array}{l}10 \\
7.5 \\
8.5 \\
8.6 \\
10\end{array}$ & $\begin{array}{l}.00 \\
.01 \\
.00 \\
.00 \\
.01\end{array}$ & $\begin{array}{l}10 \\
7.5 \\
8.5 \\
8.6 \\
10\end{array}$ \\
\hline $\begin{array}{l}78-03-09 \\
78-06-20\end{array}$ & $\begin{array}{l}36.35 \\
36.68\end{array}$ & $\begin{array}{l}78-03-08 \\
78-06-20\end{array}$ & $\begin{array}{l}1.4 \\
1.5\end{array}$ & $\begin{array}{l}28 \\
24\end{array}$ & $\begin{array}{l}1600 \\
1700\end{array}$ & $\begin{array}{l}11 \\
8.0\end{array}$ & $\begin{array}{l}.00 \\
.02\end{array}$ & $\begin{array}{l}11 \\
8.0\end{array}$ \\
\hline
\end{tabular}


analyses of water from observation wells--Continued

Depth is $42 \mathrm{ft}$. Interval open to the aquifer is 40 to $42 \mathrm{ft}$ ]

\begin{tabular}{|c|c|c|c|c|c|c|c|c|}
\hline $\begin{array}{l}\text { MAGNE- } \\
\text { SIUM, } \\
\text { DIS- } \\
\text { SOLVED } \\
\text { (MG/L } \\
\text { AS MG) }\end{array}$ & $\begin{array}{l}\text { SODIUM, } \\
\text { DIS- } \\
\text { SOLVED } \\
\text { (MG/L } \\
\text { AS NA) }\end{array}$ & $\begin{array}{l}\text { SODIUM } \\
\text { AD- } \\
\text { SORP- } \\
\text { TION } \\
\text { RATIO }\end{array}$ & $\begin{array}{l}\text { POTAS- } \\
\text { SIUM, } \\
\text { DIS- } \\
\text { SOLVED } \\
\text { (MG/L } \\
\text { AS K) }\end{array}$ & $\begin{array}{l}\text { BICAR- } \\
\text { BONATE } \\
\text { (MG/L } \\
\text { AS } \\
\text { HCO3) }\end{array}$ & $\begin{array}{c}\text { CAR- } \\
\text { BONATE } \\
\text { (MG/L } \\
\text { AS CO3) }\end{array}$ & $\begin{array}{l}\text { ALKA- } \\
\text { LINITY } \\
\text { (MG/L } \\
\text { AS } \\
\text { CACO3) }\end{array}$ & $\begin{array}{c}\text { SULFATE, } \\
\text { DIS- } \\
\text { SOLVED } \\
\text { (MG/L } \\
\text { AS SO4) }\end{array}$ & $\begin{array}{l}\text { CHLO- } \\
\text { RIDE, } \\
\text { DIS- } \\
\text { SOLVED } \\
\text { (MG/L } \\
\text { AS CL) }\end{array}$ \\
\hline $\begin{array}{l}86 \\
84 \\
84 \\
86 \\
87\end{array}$ & $\begin{array}{l}210 \\
200 \\
210 \\
220 \\
220\end{array}$ & $\begin{array}{l}3.0 \\
2.9 \\
3.1 \\
3.2 \\
3.2\end{array}$ & $\begin{array}{l}6.3 \\
6.8 \\
6.4 \\
5.6 \\
8.2\end{array}$ & $\begin{array}{l}391 \\
397 \\
398 \\
405 \\
404\end{array}$ & $\begin{array}{r}0 \\
0 \\
0 \\
-- \\
--\end{array}$ & $\begin{array}{l}321 \\
326 \\
326 \\
332 \\
331\end{array}$ & $\begin{array}{l}880 \\
840 \\
780 \\
830 \\
840\end{array}$ & $\begin{array}{l}86 \\
95 \\
88 \\
94 \\
96\end{array}$ \\
\hline $\begin{array}{l}88 \\
77 \\
88 \\
91 \\
84\end{array}$ & $\begin{array}{l}210 \\
230 \\
220 \\
220 \\
220\end{array}$ & $\begin{array}{l}3.0 \\
3.4 \\
3.1 \\
3.2 \\
3.2\end{array}$ & $\begin{array}{l}7.5 \\
4.7 \\
6.2 \\
6.8 \\
6.4\end{array}$ & $\begin{array}{l}397 \\
408 \\
419 \\
427 \\
457\end{array}$ & $\begin{array}{c}-- \\
-- \\
--\end{array}$ & $\begin{array}{l}326 \\
335 \\
344 \\
350 \\
375\end{array}$ & $\begin{array}{l}900 \\
860 \\
830 \\
880 \\
770\end{array}$ & $\begin{array}{l}90 \\
82 \\
85 \\
97 \\
94\end{array}$ \\
\hline $\begin{array}{l}87 \\
84 \\
86 \\
87 \\
89\end{array}$ & $\begin{array}{l}210 \\
230 \\
210 \\
230 \\
220\end{array}$ & $\begin{array}{l}3.0 \\
3.3 \\
3.0 \\
3.4 \\
3.2\end{array}$ & $\begin{array}{l}7.2 \\
6.9 \\
7.6 \\
6.9 \\
7.0\end{array}$ & $\begin{array}{l}457 \\
485 \\
470 \\
446 \\
466\end{array}$ & $\begin{array}{l}0 \\
0 \\
0 \\
0 \\
0\end{array}$ & $\begin{array}{l}375 \\
398 \\
386 \\
366 \\
382\end{array}$ & $\begin{array}{l}800 \\
860 \\
760 \\
850 \\
880\end{array}$ & $\begin{array}{l}87 \\
80 \\
80 \\
87 \\
90\end{array}$ \\
\hline $\begin{array}{l}87 \\
85 \\
89 \\
97 \\
93\end{array}$ & $\begin{array}{l}220 \\
230 \\
230 \\
220 \\
220\end{array}$ & $\begin{array}{l}3.2 \\
3.3 \\
3.3 \\
3.1 \\
3.1\end{array}$ & $\begin{array}{l}6.9 \\
6.9 \\
6.8 \\
6.5 \\
6.5\end{array}$ & $\begin{array}{l}439 \\
491 \\
450 \\
510 \\
450\end{array}$ & $\begin{array}{l}0 \\
0 \\
0 \\
0 \\
0\end{array}$ & $\begin{array}{l}360 \\
403 \\
369 \\
420 \\
370\end{array}$ & $\begin{array}{l}830 \\
790 \\
810 \\
800 \\
820\end{array}$ & $\begin{array}{l}94 \\
96 \\
94 \\
92 \\
95\end{array}$ \\
\hline $\begin{array}{l}94 \\
94\end{array}$ & $\begin{array}{l}210 \\
220\end{array}$ & $\begin{array}{l}2.9 \\
3.2\end{array}$ & $\begin{array}{l}7.4 \\
6.8\end{array}$ & $\begin{array}{r}91 \\
460\end{array}$ & $\begin{array}{l}0 \\
0\end{array}$ & $\begin{array}{r}75 \\
380\end{array}$ & $\begin{array}{l}830 \\
790\end{array}$ & $\begin{array}{l}110 \\
100\end{array}$ \\
\hline $\begin{array}{l}\text { NITRO- } \\
\text { GEN, } \\
\text { AMYNONIA } \\
\text { DIS- } \\
\text { SOLVED } \\
\text { (MG/L } \\
\text { AS N) }\end{array}$ & $\begin{array}{l}\text { NITRO- } \\
\text { GEN,AM- } \\
\text { MONIA + } \\
\text { ORGANIC } \\
\text { DIS. } \\
(M G / L \\
\text { AS N) }\end{array}$ & $\begin{array}{l}\text { PHOS- } \\
\text { PHORUS, } \\
\text { ORTHO, } \\
\text { DIS- } \\
\text { SOLVED } \\
\text { (MG/L } \\
\text { AS P) }\end{array}$ & $\begin{array}{l}\text { IRON, } \\
\text { DIS- } \\
\text { SOLVED } \\
\text { (UG/L } \\
\text { AS FE) }\end{array}$ & $\begin{array}{l}\text { MANGA- } \\
\text { NESE, } \\
\text { DIS- } \\
\text { SOLVED } \\
\text { (UG/L } \\
\text { AS MN) }\end{array}$ & $\begin{array}{l}\text { CARBON, } \\
\text { ORGANIC } \\
\text { DIS- } \\
\text { SOLVED } \\
\text { (MG/L } \\
\text { AS C) }\end{array}$ & & & \\
\hline $\begin{array}{l}.02 \\
.03 \\
.02 \\
.- \\
.04\end{array}$ & $\begin{array}{l}.33 \\
.53 \\
.75 \\
.- \\
.51\end{array}$ & $\begin{array}{l}.04 \\
.01 \\
.02 \\
.08 \\
.07\end{array}$ & $\begin{array}{l}30 \\
10 \\
20 \\
20 \\
30\end{array}$ & $\begin{array}{l}0 \\
0 \\
0 \\
0 \\
0\end{array}$ & $\begin{array}{l}-- \\
-- \\
-- \\
--\end{array}$ & & & \\
\hline $\begin{array}{l}.03 \\
.01 \\
.03 \\
.01 \\
.00\end{array}$ & $\begin{array}{r}.37 \\
.54 \\
.37 \\
.49 \\
.32\end{array}$ & $\begin{array}{l}.03 \\
.03 \\
.05 \\
.03 \\
.06\end{array}$ & $\begin{array}{l}50 \\
20 \\
50 \\
30 \\
90\end{array}$ & $\begin{array}{r}0 \\
0 \\
0 \\
0 \\
10\end{array}$ & $\begin{array}{l}-- \\
-- \\
-- \\
--\end{array}$ & & & \\
\hline $\begin{array}{l}.00 \\
.01 \\
.03 \\
.00 \\
.00\end{array}$ & $\begin{array}{l}.30 \\
1.1 \\
.49 \\
.42 \\
.68\end{array}$ & $\begin{array}{l}.04 \\
.06 \\
.03 \\
.06 \\
.04\end{array}$ & $\begin{array}{r}280 \\
70 \\
80 \\
130 \\
100\end{array}$ & $\begin{array}{r}0 \\
0 \\
0 \\
0 \\
10\end{array}$ & $\begin{array}{l}-- \\
-- \\
-- \\
3.9 \\
3.6\end{array}$ & & & \\
\hline $\begin{array}{l}.10 \\
.01 \\
.00 \\
.00 \\
.02\end{array}$ & $\begin{array}{l}.47 \\
1.5 \\
.64 \\
.40 \\
.21\end{array}$ & $\begin{array}{l}.02 \\
.06 \\
.04 \\
.04 \\
.02\end{array}$ & $\begin{array}{r}70 \\
130 \\
130 \\
170 \\
110\end{array}$ & $\begin{array}{r}0 \\
10 \\
10 \\
10 \\
8\end{array}$ & $\begin{array}{l}-- \\
-- \\
-- \\
--\end{array}$ & & & \\
\hline $\begin{array}{l}.00 \\
.01\end{array}$ & $\stackrel{.74}{1.5}$ & $\begin{array}{l}.01 \\
.03\end{array}$ & $\begin{array}{l}110 \\
180\end{array}$ & $\begin{array}{r}10 \\
5\end{array}$ & -- & & & \\
\hline
\end{tabular}


Table 5.--Water-level records and chemical [We11 number 16 on figure 2. Local well number is SB00506423DCB4.

\begin{tabular}{|c|c|c|c|c|c|c|c|c|}
\hline $\begin{array}{l}\text { DATE OF } \\
\text { WATER-LEVEL } \\
\text { MEASUREMENT }\end{array}$ & $\begin{array}{l}\text { WATER } \\
\text { LEVEL } \\
\text { (FEET } \\
\text { BELOW } \\
\text { LAND } \\
\text { SURFACE) }\end{array}$ & $\begin{array}{c}\text { DATE } \\
\text { OF } \\
\text { SAMPLE }\end{array}$ & $\begin{array}{l}\text { SPE- } \\
\text { CIFIC } \\
\text { CON- } \\
\text { DUCT- } \\
\text { ANCE } \\
\text { (MICRO- } \\
\text { MHOS) }\end{array}$ & $\begin{array}{c}\text { PH } \\
\text { (UNITS) }\end{array}$ & $\begin{array}{l}\text { TEMPER- } \\
\text { ATURE } \\
\text { (DEG C) }\end{array}$ & $\begin{array}{l}\text { HARD- } \\
\text { NESS } \\
\text { (MG } / L \\
\text { AS } \\
\text { CACO3) }\end{array}$ & $\begin{array}{l}\text { HARD- } \\
\text { NESS, } \\
\text { NONCAR- } \\
\text { BONATE } \\
\text { (MG/L } \\
\text { CACO3) }\end{array}$ & $\begin{array}{l}\text { CALCIUM, } \\
\text { DIS- } \\
\text { SOLVED } \\
\text { (MG/L } \\
\text { AS CA) }\end{array}$ \\
\hline $\begin{array}{l}74-05-08 \\
74-06-13 \\
74-07-23 \\
74-08-27\end{array}$ & $\begin{array}{l}36.50 \\
36.68 \\
36.60 \\
36.08\end{array}$ & $\begin{array}{l}74-04-03 \\
74-05-08 \\
74-05-13 \\
74-07-23 \\
74-08-27\end{array}$ & $\begin{array}{l}2480 \\
2440 \\
2460 \\
2470 \\
2460\end{array}$ & $\begin{array}{r}7.3 \\
7.3 \\
7.4 \\
7.3 \\
--\end{array}$ & $\begin{array}{l}12.5 \\
13.5 \\
13.5 \\
14.0 \\
13.0\end{array}$ & $\begin{array}{l}990 \\
990 \\
990 \\
960 \\
980\end{array}$ & $\begin{array}{l}650 \\
650 \\
650 \\
620 \\
640\end{array}$ & $\begin{array}{l}230 \\
230 \\
230 \\
220 \\
210\end{array}$ \\
\hline $\begin{array}{l}74-10-03 \\
74-11-11 \\
74-12-05 \\
75-02-06 \\
75-05-14\end{array}$ & $\begin{array}{l}35.68 \\
35.79 \\
35.94 \\
36.40 \\
36.94\end{array}$ & $\begin{array}{l}74-10-03 \\
74-11-11 \\
74-12-05 \\
75-02-06 \\
75-05-14\end{array}$ & $\begin{array}{l}2440 \\
2420 \\
2600 \\
2600 \\
3100\end{array}$ & $\begin{array}{l}-- \\
7.3 \\
7.0 \\
7.1 \\
7.2\end{array}$ & $\begin{array}{l}13.0 \\
12.5 \\
13.0 \\
12.0 \\
13.0\end{array}$ & $\begin{array}{r}960 \\
950 \\
990 \\
1000 \\
970\end{array}$ & $\begin{array}{l}630 \\
620 \\
650 \\
650 \\
630\end{array}$ & $\begin{array}{l}220 \\
230 \\
230 \\
240 \\
230\end{array}$ \\
\hline $\begin{array}{l}75-08-11 \\
75-12-01 \\
76-03-29 \\
76-06-29 \\
76-09-22\end{array}$ & $\begin{array}{l}36.56 \\
35.68 \\
36.66 \\
37.05 \\
36.01\end{array}$ & $\begin{array}{l}75-08-11 \\
75-12-01 \\
76-03-29 \\
76-06-29 \\
76-09-22\end{array}$ & $\begin{array}{l}3100 \\
2650 \\
2200 \\
2400 \\
2200\end{array}$ & $\begin{array}{l}7.2 \\
7.0 \\
7.6 \\
7.0 \\
7.2\end{array}$ & $\begin{array}{l}14.0 \\
13.0 \\
12.0 \\
13.0 \\
13.0\end{array}$ & $\begin{array}{l}940 \\
940 \\
900 \\
940 \\
930\end{array}$ & $\begin{array}{l}600 \\
610 \\
560 \\
620 \\
600\end{array}$ & $\begin{array}{l}210 \\
220 \\
200 \\
220 \\
210\end{array}$ \\
\hline $\begin{array}{l}76-12-06 \\
77-03-08 \\
77-06-15 \\
77-09-13 \\
77-12-06\end{array}$ & $\begin{array}{l}35.40 \\
36.56 \\
36.20 \\
36.79 \\
35.40\end{array}$ & $\begin{array}{l}76-12-08 \\
77-03-08 \\
77-06-15 \\
77-09-12 \\
77-12-06\end{array}$ & $\begin{array}{l}2200 \\
2300 \\
2280 \\
2200 \\
2200\end{array}$ & $\begin{array}{l}7.3 \\
7.2 \\
7.5 \\
7.4 \\
7.1\end{array}$ & $\begin{array}{l}12.0 \\
13.0 \\
13.5 \\
13.0 \\
12.0\end{array}$ & $\begin{array}{l}920 \\
910 \\
880 \\
920 \\
920\end{array}$ & $\begin{array}{l}590 \\
580 \\
560 \\
580 \\
580\end{array}$ & $\begin{array}{l}210 \\
210 \\
200 \\
210 \\
210\end{array}$ \\
\hline $\begin{array}{l}78-03-09 \\
78-06-20\end{array}$ & $\begin{array}{l}36.45 \\
36.88\end{array}$ & $\begin{array}{l}78-03-09 \\
78-06-20\end{array}$ & $\begin{array}{l}2200 \\
2300\end{array}$ & $\begin{array}{l}7.1 \\
7.3\end{array}$ & $\begin{array}{l}13.0 \\
13.0\end{array}$ & $\begin{array}{l}930 \\
900\end{array}$ & $\begin{array}{l}890 \\
570\end{array}$ & $\begin{array}{l}210 \\
200\end{array}$ \\
\hline $\begin{array}{l}\text { DATE OF } \\
\text { WATER-LEVEL } \\
\text { MEASUREMENT }\end{array}$ & $\begin{array}{l}\text { WATER } \\
\text { LEVEL } \\
\text { (FEET } \\
\text { BELOW } \\
\text { LAND } \\
\text { SURFACE) }\end{array}$ & $\begin{array}{c}\text { DATE } \\
\text { OF } \\
\text { SAMPLEE }\end{array}$ & $\begin{array}{l}\text { FLUO- } \\
\text { RIDE, } \\
\text { DIS- } \\
\text { SOLVED } \\
\text { (MG/L } \\
\text { AS F) }\end{array}$ & $\begin{array}{l}\text { SILICA, } \\
\text { DIS- } \\
\text { SOLVED } \\
\text { (MG/L } \\
\text { AS } \\
\text { SI02) }\end{array}$ & $\begin{array}{c}\text { SOLIDS, } \\
\text { SUM OF } \\
\text { CONSTI- } \\
\text { TUENTS, } \\
\text { DIS- } \\
\text { SOLVED } \\
\text { (MG/L) }\end{array}$ & $\begin{array}{l}\text { NITRO- } \\
\text { GEN, } \\
\text { NITRATE } \\
\text { DIS- } \\
\text { SOLVED } \\
(M G / L \\
\text { AS N) }\end{array}$ & $\begin{array}{l}\text { NITRO- } \\
\text { GEN, } \\
\text { NITRITE } \\
\text { DIS- } \\
\text { SOLVED } \\
\text { (MG/L } \\
\text { AS N) }\end{array}$ & $\begin{array}{l}\text { NITRO- } \\
\text { GEN, } \\
\text { NO2+ NO3 } \\
\text { DIS- } \\
\text { SOLVED } \\
\text { (MG/L } \\
\text { AS N) }\end{array}$ \\
\hline $\begin{array}{l}74-05-08 \\
74-06-13 \\
74-07-23 \\
74-08-27\end{array}$ & $\begin{array}{l}36.50 \\
36.68 \\
36.60 \\
36.08\end{array}$ & $\begin{array}{l}74-04-03 \\
74-05-08 \\
74-06-13 \\
74-07-23 \\
74-08-27\end{array}$ & $\begin{array}{l}1.8 \\
2.0 \\
2.3 \\
1.9 \\
1.9\end{array}$ & $\begin{array}{l}23 \\
23 \\
28 \\
24 \\
24\end{array}$ & $\begin{array}{l}1800 \\
1830 \\
1840 \\
1800 \\
1780\end{array}$ & $\begin{array}{l}8.6 \\
8.6 \\
7.5 \\
-- \\
--\end{array}$ & $\begin{array}{l}.01 \\
.00 \\
.00 \\
. \overline{01}\end{array}$ & $\begin{array}{l}8.6 \\
8.6 \\
7.5 \\
9.0 \\
9.3\end{array}$ \\
\hline $\begin{array}{l}74-10-03 \\
74-11-11 \\
74-12-05 \\
75-02-06 \\
75-05-14\end{array}$ & $\begin{array}{l}35.68 \\
35.79 \\
35.94 \\
36.40 \\
36.94\end{array}$ & $\begin{array}{l}74-10-03 \\
74-11-11 \\
74-12-05 \\
75-02-06 \\
75-05-14\end{array}$ & $\begin{array}{l}1.8 \\
1.5 \\
1.8 \\
1.7 \\
1.9\end{array}$ & $\begin{array}{l}24 \\
24 \\
23 \\
24 \\
23\end{array}$ & $\begin{array}{l}1810 \\
1800 \\
1800 \\
2290 \\
1790\end{array}$ & $\begin{array}{l}9.4 \\
10^{\circ} \\
9.2 \\
10 \\
10\end{array}$ & $\begin{array}{l}.01 \\
.01 \\
.00 \\
.00 \\
.00\end{array}$ & $\begin{array}{c}9.4 \\
10 \\
9.2 \\
10 \\
10\end{array}$ \\
\hline $\begin{array}{l}75-08-11 \\
75-12-01 \\
76-03-29 \\
76-06-29 \\
76-09-22\end{array}$ & $\begin{array}{l}36.56 \\
35.68 \\
36.66 \\
37.05 \\
36.01\end{array}$ & $\begin{array}{l}75-08-11 \\
75-12-01 \\
76-03-29 \\
76-06-29 \\
76-09-22\end{array}$ & $\begin{array}{l}1.7 \\
1.8 \\
1.8 \\
1.9 \\
1.9\end{array}$ & $\begin{array}{l}23 \\
22 \\
22 \\
22 \\
22\end{array}$ & $\begin{array}{l}1830 \\
1840 \\
1740 \\
1820 \\
1810\end{array}$ & $\begin{array}{l}9.8 \\
9.4 \\
10 \\
10 \\
10\end{array}$ & $\begin{array}{l}.00 \\
.01 \\
.00 \\
.00 \\
.01\end{array}$ & $\begin{array}{l}9.8 \\
9.4 \\
10 \\
10 \\
10\end{array}$ \\
\hline $\begin{array}{l}76-12-06 \\
77-03-08 \\
77-06-15 \\
77-09-13 \\
77-12-06\end{array}$ & $\begin{array}{l}35.40 \\
36.56 \\
36.20 \\
36.79 \\
35.40\end{array}$ & $\begin{array}{l}76-12-08 \\
77-03-08 \\
77-06-15 \\
77-09-12 \\
77-12-06\end{array}$ & $\begin{array}{l}1.9 \\
1.9 \\
1.9 \\
1.9 \\
1.9\end{array}$ & $\begin{array}{l}22 \\
24 \\
22 \\
24 \\
23\end{array}$ & $\begin{array}{l}1800 \\
1750 \\
1690 \\
1720 \\
1720\end{array}$ & $\begin{array}{l}14 \\
9.8 \\
9.7 \\
9.8 \\
11\end{array}$ & $\begin{array}{l}.00 \\
.00 \\
.00 \\
.00 \\
.01\end{array}$ & $\begin{array}{c}14 \\
9.8 \\
9.7 \\
9.8 \\
11\end{array}$ \\
\hline $\begin{array}{l}78-03-09 \\
78-06-20\end{array}$ & $\begin{array}{l}36.45 \\
36.88\end{array}$ & $\begin{array}{l}78-03-09 \\
78-06-20\end{array}$ & $\begin{array}{l}1.1 \\
1.9\end{array}$ & $\begin{array}{l}21 \\
19\end{array}$ & $\begin{array}{l}1530 \\
1670\end{array}$ & $\begin{array}{c}11 \\
9.2\end{array}$ & $\begin{array}{l}.00 \\
.02\end{array}$ & $\begin{array}{c}11 \\
9.2\end{array}$ \\
\hline
\end{tabular}


analyses of water from observation wells--Continued

Depth is $60 \mathrm{ft}$. Interval open to the aquifer is 56 to $60 \mathrm{ft}$ ]

\begin{tabular}{|c|c|c|c|c|c|c|c|c|}
\hline $\begin{array}{l}\text { MAGNE- } \\
\text { SILM, } \\
\text { DIS- } \\
\text { SO!VED } \\
\text { (MG/L } \\
\text { AS MG) }\end{array}$ & $\begin{array}{l}\text { SODILM! } \\
\text { DIS- } \\
\text { SOLVED } \\
\text { (MG/L } \\
\text { AS NA) }\end{array}$ & $\begin{array}{l}\text { SCDIUM } \\
\text { AD- } \\
\text { SORP- } \\
\text { TION } \\
\text { RATIO }\end{array}$ & $\begin{array}{l}\text { POTAS- } \\
\text { SIUM, } \\
\text { DIS- } \\
\text { SOLVED } \\
\text { (MG/L } \\
\text { AS K) }\end{array}$ & $\begin{array}{l}\text { BICAR- } \\
\text { BONATE } \\
\text { (MG/L } \\
\text { AS } \\
\text { HCO3) }\end{array}$ & $\begin{array}{c}\text { CAR- } \\
\text { BONATE } \\
(\mathrm{MG} / \mathrm{L} \\
\text { AS C03) }\end{array}$ & $\begin{array}{l}\text { ALKA- } \\
\text { LINITY } \\
\text { (MG/L } \\
\text { AS } \\
\text { CAC03) }\end{array}$ & $\begin{array}{c}\text { SULFATE, } \\
\text { DIS- } \\
\text { SOLVED } \\
\text { (MG/L } \\
\text { AS SO4) }\end{array}$ & $\begin{array}{l}\text { CHLO- } \\
\text { RIDE, } \\
\text { DIS- } \\
\text { SOLVED } \\
(H G / L \\
\text { AS CL) }\end{array}$ \\
\hline $\begin{array}{l}100 \\
100 \\
100 \\
100 \\
110\end{array}$ & $\begin{array}{l}220 \\
210 \\
220 \\
220 \\
210\end{array}$ & $\begin{array}{l}3.1 \\
2.9 \\
3.1 \\
3.1 \\
2.9\end{array}$ & $\begin{array}{l}6.9 \\
6.3 \\
5.8 \\
5.3 \\
7.2\end{array}$ & $\begin{array}{l}411 \\
411 \\
409 \\
410 \\
408\end{array}$ & $\begin{array}{r}0 \\
0 \\
0 \\
-- \\
--\end{array}$ & $\begin{array}{l}337 \\
337 \\
335 \\
336 \\
335\end{array}$ & $\begin{array}{l}870 \\
910 \\
910 \\
880 \\
850\end{array}$ & $\begin{array}{l}110 \\
110 \\
110 \\
110 \\
120\end{array}$ \\
\hline $\begin{array}{r}100 \\
91 \\
100 \\
100 \\
95\end{array}$ & $\begin{array}{l}220 \\
220 \\
220 \\
350 \\
220\end{array}$ & $\begin{array}{l}3.1 \\
3.1 \\
3.1 \\
4.8 \\
3.1\end{array}$ & $\begin{array}{l}6.8 \\
3.3 \\
5.8 \\
6.4 \\
5.8\end{array}$ & $\begin{array}{l}409 \\
407 \\
409 \\
445 \\
409\end{array}$ & $\begin{array}{l}-- \\
-- \\
-- \\
0\end{array}$ & $\begin{array}{l}336 \\
334 \\
335 \\
365 \\
335\end{array}$ & $\begin{array}{r}880 \\
880 \\
860 \\
1200 \\
860\end{array}$ & $\begin{array}{l}110 \\
110 \\
120 \\
100 \\
110\end{array}$ \\
\hline $\begin{array}{r}100 \\
95 \\
97 \\
96 \\
99\end{array}$ & $\begin{array}{l}220 \\
230 \\
210 \\
230 \\
220\end{array}$ & $\begin{array}{l}3.1 \\
3.3 \\
3.0 \\
3.3 \\
3.1\end{array}$ & $\begin{array}{l}6.6 \\
6.5 \\
6.7 \\
6.3 \\
6.2\end{array}$ & $\begin{array}{l}406 \\
398 \\
402 \\
402 \\
401\end{array}$ & $\begin{array}{l}0 \\
0 \\
0 \\
0 \\
0\end{array}$ & $\begin{array}{l}333 \\
326 \\
335 \\
330 \\
329\end{array}$ & $\begin{array}{l}900 \\
910 \\
850 \\
880 \\
900\end{array}$ & $\begin{array}{l}120 \\
120 \\
110 \\
120 \\
110\end{array}$ \\
\hline $\begin{array}{l}96 \\
94 \\
93 \\
96 \\
96\end{array}$ & $\begin{array}{l}220 \\
220 \\
220 \\
220 \\
220\end{array}$ & $\begin{array}{l}3.2 \\
3.2 \\
3.2 \\
3.2 \\
3.2\end{array}$ & $\begin{array}{l}6.3 \\
6.0 \\
5.9 \\
5.6 \\
6.3\end{array}$ & $\begin{array}{l}399 \\
401 \\
390 \\
410 \\
410\end{array}$ & $\begin{array}{l}0 \\
0 \\
0 \\
0 \\
0\end{array}$ & $\begin{array}{l}327 \\
329 \\
320 \\
340 \\
340\end{array}$ & $\begin{array}{l}860 \\
840 \\
800 \\
810 \\
800\end{array}$ & $\begin{array}{l}120 \\
110 \\
110 \\
110 \\
110\end{array}$ \\
\hline $\begin{array}{l}98 \\
93\end{array}$ & $\begin{array}{l}210 \\
220\end{array}$ & $\begin{array}{l}3.0 \\
3.2\end{array}$ & $\begin{array}{l}6.3 \\
6.0\end{array}$ & $\begin{array}{r}48 \\
410\end{array}$ & $\begin{array}{l}0 \\
0\end{array}$ & $\begin{array}{r}39 \\
340\end{array}$ & $\begin{array}{l}800 \\
760\end{array}$ & $\begin{array}{l}110 \\
120\end{array}$ \\
\hline $\begin{array}{l}\text { NITRO- } \\
\text { GEN, } \\
\text { AMMONIA } \\
\text { DIS- } \\
\text { SOLVED } \\
(M G / L \\
\text { AS N) }\end{array}$ & $\begin{array}{l}\text { NITRO- } \\
\text { GEN,AM- } \\
\text { MONIA + } \\
\text { ORGANIC } \\
\text { DIS. } \\
(M G / L \\
\text { AS N) }\end{array}$ & $\begin{array}{c}\text { PHOS- } \\
\text { PHORUS, } \\
\text { ORTHO, } \\
\text { DIS- } \\
\text { SOLVED } \\
\text { (MG/L } \\
\text { AS P) }\end{array}$ & $\begin{array}{l}\text { IRON, } \\
\text { DIS- } \\
\text { SOLVED } \\
\text { (UG/L } \\
\text { AS FE) }\end{array}$ & $\begin{array}{l}\text { MANGA- } \\
\text { NESE, } \\
\text { DIS- } \\
\text { SOLVED } \\
\text { (UG/L } \\
\text { AS MN) }\end{array}$ & $\begin{array}{l}\text { CARBON, } \\
\text { ORGANIC } \\
\text { DIS- } \\
\text { SOLVED } \\
\text { (MG/L } \\
\text { AS C) }\end{array}$ & & & \\
\hline $\begin{array}{l}.02 \\
.03 \\
.03 \\
.- \\
.04\end{array}$ & $\begin{array}{r}.58 \\
.59 \\
.63 \\
.- \\
.48\end{array}$ & $\begin{array}{l}.04 \\
.01 \\
.01 \\
.08 \\
.06\end{array}$ & $\begin{array}{r}100 \\
10 \\
20 \\
20 \\
20\end{array}$ & $\begin{array}{r}0 \\
10 \\
0 \\
0 \\
20\end{array}$ & $\begin{array}{l}-- \\
-- \\
-- \\
--\end{array}$ & & & \\
\hline $\begin{array}{l}.03 \\
.03 \\
.04 \\
.01 \\
.00\end{array}$ & $\begin{array}{l}.43 \\
.57 \\
.42 \\
.52 \\
.08\end{array}$ & $\begin{array}{l}.04 \\
.04 \\
.04 \\
.03 \\
.06\end{array}$ & $\begin{array}{l}20 \\
10 \\
20 \\
40 \\
20\end{array}$ & $\begin{array}{r}30 \\
0 \\
0 \\
150 \\
10\end{array}$ & $\begin{array}{l}-- \\
-- \\
-- \\
--\end{array}$ & & & \\
\hline $\begin{array}{l}.00 \\
.04 \\
.02 \\
.00 \\
.00\end{array}$ & $\begin{array}{l}.67 \\
.99 \\
.48 \\
.42 \\
.52\end{array}$ & $\begin{array}{l}.06 \\
.05 \\
.04 \\
.06 \\
.05\end{array}$ & $\begin{array}{l}10 \\
40 \\
10 \\
20 \\
10\end{array}$ & $\begin{array}{r}0 \\
5 \\
0 \\
0 \\
10\end{array}$ & $\begin{array}{l}-. \\
-- \\
3.7 \\
5.1\end{array}$ & & & \\
\hline $\begin{array}{l}.06 \\
.00 \\
.00 \\
.01 \\
.06\end{array}$ & $\begin{array}{r}.53 \\
.84 \\
.35 \\
.00 \\
1.4\end{array}$ & $\begin{array}{l}.03 \\
.06 \\
.05 \\
.04 \\
.02\end{array}$ & $\begin{array}{l}20 \\
30 \\
20 \\
20 \\
50\end{array}$ & $\begin{array}{l}0 \\
0 \\
0 \\
0 \\
4\end{array}$ & $\begin{array}{l}-- \\
-- \\
-- \\
--\end{array}$ & & & \\
\hline $\begin{array}{l}.00 \\
.01\end{array}$ & $.7^{72}$ & $\begin{array}{l}.42 \\
.03\end{array}$ & $\begin{array}{r}20 \\
0\end{array}$ & $\begin{array}{l}10 \\
10\end{array}$ & -- & & & \\
\hline
\end{tabular}


Table 5.--Water-level records and chemical [We11 number 18 on figure 2. Local we11 number is SB00506424CCA1.

\begin{tabular}{|c|c|c|c|c|c|c|c|c|}
\hline $\begin{array}{l}\text { DATE OF } \\
\text { WATER-LEVEL } \\
\text { MEASUREMENT }\end{array}$ & $\begin{array}{l}\text { WATER } \\
\text { LEVEL } \\
\text { (FEET } \\
\text { BELOW } \\
\text { LAND } \\
\text { SURFACE) }\end{array}$ & $\begin{array}{c}\text { DATE } \\
\text { OF } \\
\text { SAMPLE }\end{array}$ & $\begin{array}{l}\text { SPE- } \\
\text { CIFIC } \\
\text { CON- } \\
\text { DUCT- } \\
\text { ANCE } \\
\text { (MICRO- } \\
\text { MHOS) }\end{array}$ & $\begin{array}{c}\text { PH } \\
\text { (UNITS) }\end{array}$ & $\begin{array}{l}\text { TEMPER- } \\
\text { ATURE } \\
\text { (DEG C) }\end{array}$ & $\begin{array}{l}\text { HARD- } \\
\text { NESS } \\
\text { (MG/L } \\
\text { AS } \\
\text { CAC03) }\end{array}$ & $\begin{array}{l}\text { HARD- } \\
\text { NESS, } \\
\text { NONCAR- } \\
\text { BONATE } \\
\text { (MG/L } \\
\text { CAC03) }\end{array}$ & $\begin{array}{l}\text { CALCIUM, } \\
\text { DIS- } \\
\text { SOLYED } \\
(M G / L \\
\text { AS CA) }\end{array}$ \\
\hline $\begin{array}{l}74-03-25 \\
74-04-04 \\
74-05-08 \\
74-06-14 \\
74-07-23\end{array}$ & $\begin{array}{l}32.50 \\
32.60 \\
32.98 \\
33.09 \\
33.30\end{array}$ & $\begin{array}{l}74-04-04 \\
74-05-09 \\
74-06-14 \\
74-07-24\end{array}$ & $\begin{array}{l}2240 \\
2240 \\
2200 \\
2210\end{array}$ & $\begin{array}{l}--- \\
6.9 \\
7.3 \\
7.4 \\
7.5\end{array}$ & $\begin{array}{l}13 . \overline{0} \\
13.5 \\
14.0 \\
14.5\end{array}$ & $\begin{array}{l}-130 \\
790 \\
820 \\
820\end{array}$ & $\begin{array}{l}-\cdots \\
500 \\
520 \\
520\end{array}$ & $\begin{array}{l}210 \\
200 \\
210 \\
210\end{array}$ \\
\hline $\begin{array}{l}74-08-27 \\
74-10-03 \\
74-11-11 \\
74-12-05 \\
75-02-06\end{array}$ & $\begin{array}{l}33.23 \\
32.90 \\
32.73 \\
32.81 \\
33.05\end{array}$ & $\begin{array}{l}74-08-28 \\
74-10-03 \\
74-11-11 \\
74-12-05 \\
75-02-06\end{array}$ & $\begin{array}{l}2200 \\
2200 \\
2300 \\
2400 \\
2400\end{array}$ & $\begin{array}{l}-- \\
-- \\
7.2 \\
7.2 \\
7.1\end{array}$ & $\begin{array}{l}14.0 \\
13.5 \\
13.5 \\
13.0 \\
13.0\end{array}$ & $\begin{array}{l}800 \\
840 \\
820 \\
830 \\
820\end{array}$ & $\begin{array}{l}500 \\
540 \\
530 \\
530 \\
520\end{array}$ & $\begin{array}{l}200 \\
220 \\
220 \\
210 \\
210\end{array}$ \\
\hline $\begin{array}{l}75-05-14 \\
75-08-12 \\
75-12-02 \\
76-03-29 \\
76-06-29\end{array}$ & $\begin{array}{l}33.38 \\
33.50 \\
32.60 \\
33.05 \\
33.59\end{array}$ & $\begin{array}{l}75-05-14 \\
75-08-12 \\
75-12-02 \\
76-03-29 \\
76-06-29\end{array}$ & $\begin{array}{l}2800 \\
2600 \\
2700 \\
2200 \\
2200\end{array}$ & $\begin{array}{l}7.2 \\
7.5 \\
7.4 \\
7.6 \\
6.8\end{array}$ & $\begin{array}{l}14.0 \\
14.0 \\
13.5 \\
13.0 \\
14.0\end{array}$ & $\begin{array}{l}840 \\
880 \\
320 \\
810 \\
850\end{array}$ & $\begin{array}{l}550 \\
580 \\
520 \\
510 \\
540\end{array}$ & $\begin{array}{l}210 \\
230 \\
210 \\
210 \\
220\end{array}$ \\
\hline $\begin{array}{l}76-09-22 \\
76-12-08 \\
77-03-08 \\
77-06-15 \\
77-09-13\end{array}$ & $\begin{array}{l}32.97 \\
32.74 \\
33.08 \\
33.85 \\
33.83\end{array}$ & $\begin{array}{l}76-09-22 \\
76-12-08 \\
77-03-08 \\
77-06-15 \\
77-09-13\end{array}$ & $\begin{array}{l}2100 \\
2100 \\
2200 \\
2400 \\
2200\end{array}$ & $\begin{array}{l}7.3 \\
7.4 \\
7.5 \\
7.3 \\
7.4\end{array}$ & $\begin{array}{l}13.5 \\
13.5 \\
13.5 \\
14.0 \\
14.0\end{array}$ & $\begin{array}{l}830 \\
830 \\
820 \\
840 \\
830\end{array}$ & $\begin{array}{l}520 \\
530 \\
520 \\
530 \\
520\end{array}$ & $\begin{array}{l}210 \\
210 \\
210 \\
210 \\
210\end{array}$ \\
\hline $\begin{array}{l}77-12-06 \\
78-03-09 \\
78-06-20\end{array}$ & $\begin{array}{l}32.99 \\
33.10 \\
33.18\end{array}$ & $\begin{array}{l}77-12-06 \\
78-03-09 \\
78-06-20\end{array}$ & $\begin{array}{l}2200 \\
2250 \\
2200\end{array}$ & $\begin{array}{l}7.3 \\
7.3 \\
7.3\end{array}$ & $\begin{array}{l}12.5 \\
13.0 \\
14.0\end{array}$ & $\begin{array}{l}830 \\
830 \\
830\end{array}$ & $\begin{array}{l}510 \\
530 \\
530\end{array}$ & $\begin{array}{l}210 \\
210 \\
210\end{array}$ \\
\hline $\begin{array}{l}\text { DATE OF } \\
\text { WATER-LEVEL } \\
\text { MEASUREMENT }\end{array}$ & $\begin{array}{l}\text { WATER } \\
\text { LEVEL } \\
\text { (FEET } \\
\text { BELOW } \\
\text { LAND } \\
\text { SURFACE) }\end{array}$ & $\begin{array}{c}\text { DATE } \\
\text { OF } \\
\text { SAMPLE }\end{array}$ & $\begin{array}{l}\text { FLUO- } \\
\text { RIDE, } \\
\text { DIS- } \\
\text { SOLVED } \\
\text { (MG/L } \\
\text { AS F) }\end{array}$ & $\begin{array}{l}\text { SILICA, } \\
\text { DIS- } \\
\text { SOLVED } \\
\text { (IIG/L } \\
\text { AS } \\
\text { SID2) }\end{array}$ & $\begin{array}{l}\text { SOLIDS, } \\
\text { SUM OF } \\
\text { CONST I- } \\
\text { TUENTS, } \\
\text { DIS- } \\
\text { SOLVED } \\
\text { (MG/L) }\end{array}$ & $\begin{array}{l}\text { NITRO- } \\
\text { GEN, } \\
\text { NITRATE } \\
\text { DIS- } \\
\text { SOI.VED } \\
(M G / L \\
\text { AS N) }\end{array}$ & $\begin{array}{l}\text { NITRO- } \\
\text { GEN, } \\
\text { NITRITE } \\
\text { DIS- } \\
\text { SOLVED } \\
(M G / L \\
\text { AS N) }\end{array}$ & $\begin{array}{c}\text { NITKO- } \\
\text { GEN, } \\
\text { NO2+NO3 } \\
\text { DIS- } \\
\text { SOLVED } \\
(M G / L \\
\text { AS N) }\end{array}$ \\
\hline $\begin{array}{l}74-03-25 \\
74-04-04 \\
74-05-08 \\
74-06-14 \\
74-07-23\end{array}$ & $\begin{array}{l}32.50 \\
32.60 \\
32.98 \\
33.09 \\
33.30\end{array}$ & $\begin{array}{l}74-04-04 \\
74-05-09 \\
74-06-14 \\
74-07-24\end{array}$ & $\begin{array}{l}1 . \overline{1} \\
1.2 \\
1.1 \\
1.2\end{array}$ & $\begin{array}{l}-- \\
18 \\
17 \\
17 \\
18\end{array}$ & $\begin{array}{l}-\overline{1590} \\
1610 \\
1640 \\
1610\end{array}$ & $\begin{array}{l}3 . \overline{4}^{-} \\
4.0 \\
5.3 \\
--\end{array}$ & $\begin{array}{l}.- \\
.18 \\
.04 \\
.05 \\
--\end{array}$ & $\begin{array}{l}3.6^{--} \\
4.0 \\
5.3 \\
5.0\end{array}$ \\
\hline $\begin{array}{l}74-08-27 \\
74-10-03 \\
74-11-11 \\
74-12-05 \\
75-02-06\end{array}$ & $\begin{array}{l}33.23 \\
32.90 \\
32.73 \\
32.81 \\
33.05\end{array}$ & $\begin{array}{l}74-08-28 \\
74-10-03 \\
74-11-11 \\
74-12-05 \\
75-02-06\end{array}$ & $\begin{array}{l}1.1 \\
1.1 \\
1.0 \\
1.2 \\
1.1\end{array}$ & $\begin{array}{l}17 \\
18 \\
17 \\
17 \\
18\end{array}$ & $\begin{array}{l}1580 \\
1640 \\
1570 \\
1590 \\
1650\end{array}$ & $\begin{array}{l}-- \\
5.6 \\
5.1 \\
5.7\end{array}$ & $\begin{array}{l}.00 \\
.02 \\
.01 \\
.01 \\
.01\end{array}$ & $\begin{array}{l}4.6 \\
5.1 \\
5.6 \\
5.1 \\
5.7\end{array}$ \\
\hline $\begin{array}{l}75-05-14 \\
75-08-12 \\
75-12-02 \\
76-03-29 \\
76-06-29\end{array}$ & $\begin{array}{l}33.38 \\
33.50 \\
32.60 \\
33.05 \\
33.59\end{array}$ & $\begin{array}{l}75-05-14 \\
75-08-12 \\
75-12-02 \\
76-03-29 \\
76-06-29\end{array}$ & $\begin{array}{r}1.1 \\
1.1 \\
.8 \\
1.1 \\
1.2\end{array}$ & $\begin{array}{l}17 \\
17 \\
11 \\
16 \\
16\end{array}$ & $\begin{array}{l}1640 \\
1660 \\
1640 \\
1620 \\
1710\end{array}$ & $\begin{array}{l}5.5 \\
4.9 \\
3.6 \\
6.0 \\
5.9\end{array}$ & $\begin{array}{l}.01 \\
.01 \\
.01 \\
.00 \\
.00\end{array}$ & $\begin{array}{l}5.5 \\
4.9 \\
3.6 \\
6.0 \\
5.9\end{array}$ \\
\hline $\begin{array}{l}76-09-22 \\
76-12-08 \\
77-03-08 \\
77-06-15 \\
77-09-13\end{array}$ & $\begin{array}{l}32.97 \\
32.74 \\
33.08 \\
33.85 \\
33.83\end{array}$ & $\begin{array}{l}76-09-22 \\
76-12-08 \\
77-03-08 \\
77-06-15 \\
77-09-13\end{array}$ & $\begin{array}{l}1.2 \\
1.1 \\
1.3 \\
1.3 \\
1.2\end{array}$ & $\begin{array}{l}16 \\
16 \\
18 \\
16 \\
18\end{array}$ & $\begin{array}{l}1630 \\
1680 \\
1630 \\
1630 \\
1650\end{array}$ & $\begin{array}{l}6.5 \\
8.3 \\
6.3 \\
6.0 \\
6.5\end{array}$ & $\begin{array}{l}.01 \\
.00 \\
.01 \\
.00 \\
.00\end{array}$ & $\begin{array}{l}6.5 \\
8.3 \\
6.3 \\
6.0 \\
6.5\end{array}$ \\
\hline $\begin{array}{l}77-12-06 \\
78-03-09 \\
78-06-20\end{array}$ & $\begin{array}{l}32.99 \\
33.10 \\
33.18\end{array}$ & $\begin{array}{l}77-12-06 \\
78-03-09 \\
78-06-20\end{array}$ & $\begin{array}{l}1.2 \\
1.2 \\
1.1\end{array}$ & $\begin{array}{l}18 \\
16 \\
14\end{array}$ & $\begin{array}{l}1630 \\
1570 \\
1610\end{array}$ & $\begin{array}{l}7.9 \\
7.1 \\
6.6\end{array}$ & $\begin{array}{l}.01 \\
.00 \\
.02\end{array}$ & $\begin{array}{l}7.9 \\
7.1 \\
6.6\end{array}$ \\
\hline
\end{tabular}


analyses of water from observation wells--Continued

Depth is $113 \mathrm{ft}$. Interval open to the aquifer is 109 to $113 \mathrm{ft}$ ]

\begin{tabular}{|c|c|c|c|c|c|c|c|c|}
\hline $\begin{array}{l}\text { MAGNE- } \\
\text { SIUH, } \\
\text { DIS- } \\
\text { SOLVED } \\
\text { (MG/L } \\
\text { AS MG) }\end{array}$ & $\begin{array}{l}\text { SODIUH, } \\
\text { DIS- } \\
\text { SOLVED } \\
\text { (HSLL } \\
\text { AS NA) }\end{array}$ & $\begin{array}{l}\text { SODIUM } \\
\text { AD- } \\
\text { SORP- } \\
\text { TION } \\
\text { PATIO }\end{array}$ & $\begin{array}{l}\text { POTAS- } \\
\text { SIUM, } \\
\text { DIS- } \\
\text { SOIVED } \\
(M G / L \\
\text { AS K) }\end{array}$ & $\begin{array}{c}\text { BICAR- } \\
\text { BONATE } \\
\text { (MG/L } \\
\text { AS } \\
\text { HCO3) }\end{array}$ & $\begin{array}{c}\text { CAR- } \\
\text { BOINATE } \\
(M G / L \\
\text { AS C03) }\end{array}$ & $\begin{array}{l}\text { ALKA- } \\
\text { LINITY } \\
\text { (MG/L } \\
\text { AS } \\
\text { CACO3) }\end{array}$ & $\begin{array}{c}\text { SULFATE, } \\
\text { DIS- } \\
\text { SOLVED } \\
\text { (MG/L } \\
\text { AS SO4) }\end{array}$ & $\begin{array}{l}\text { CHL } \\
\text { RIO } \\
\text { DIS } \\
\text { SOL } \\
\text { (MG } \\
\text { AS }\end{array}$ \\
\hline $\begin{array}{l}-- \\
74 \\
71 \\
71 \\
72\end{array}$ & $\begin{array}{l}-1- \\
210 \\
210 \\
220 \\
220\end{array}$ & $\begin{array}{l}-- \\
3.2 \\
3.2 \\
3.4 \\
3.3\end{array}$ & $\begin{array}{l}6 . \overline{6} \\
5.3 \\
4.9 \\
4.5\end{array}$ & $\begin{array}{l}-1-- \\
400 \\
372 \\
359 \\
361\end{array}$ & $\begin{array}{r}-- \\
0 \\
0 \\
0 \\
--\end{array}$ & $\begin{array}{l}328 \\
305 \\
294 \\
296\end{array}$ & $\begin{array}{l}770 \\
810 \\
830 \\
790\end{array}$ & $\begin{array}{l}-- \\
89 \\
97 \\
86 \\
93\end{array}$ \\
\hline $\begin{array}{l}73 \\
71 \\
66 \\
74 \\
72\end{array}$ & $\begin{array}{l}220 \\
210 \\
210 \\
210 \\
210\end{array}$ & $\begin{array}{l}3.4 \\
3.2 \\
3.2 \\
3.2 \\
3.2\end{array}$ & $\begin{array}{l}6.3 \\
5.5 \\
3.5 \\
4.6 \\
5.1\end{array}$ & $\begin{array}{l}361 \\
363 \\
361 \\
363 \\
364\end{array}$ & $\begin{array}{l}-- \\
-- \\
-- \\
--\end{array}$ & $\begin{array}{l}296 \\
298 \\
296 \\
298 \\
299\end{array}$ & $\begin{array}{l}770 \\
820 \\
760 \\
780 \\
830\end{array}$ & $\begin{array}{r}93 \\
89 \\
94 \\
87 \\
100\end{array}$ \\
\hline $\begin{array}{l}77 \\
73 \\
71 \\
70 \\
72\end{array}$ & $\begin{array}{l}210 \\
210 \\
230 \\
210 \\
2.30\end{array}$ & $\begin{array}{l}3.2 \\
3.1 \\
3.5 \\
3.2 \\
3.4\end{array}$ & $\begin{array}{l}5.7 \\
5.7 \\
5.4 \\
5.4 \\
5.4\end{array}$ & $\begin{array}{l}352 \\
361 \\
359 \\
370 \\
368\end{array}$ & $\begin{array}{l}0 \\
0 \\
0 \\
0 \\
0\end{array}$ & $\begin{array}{l}289 \\
296 \\
294 \\
303 \\
302\end{array}$ & $\begin{array}{l}830 \\
830 \\
820 \\
810 \\
870\end{array}$ & $\begin{array}{r}88 \\
94 \\
100 \\
91 \\
86\end{array}$ \\
\hline $\begin{array}{l}73 \\
75 \\
72 \\
76 \\
75\end{array}$ & $\begin{array}{l}220 \\
220 \\
220 \\
220 \\
220\end{array}$ & $\begin{array}{l}3.3 \\
3.3 \\
3.3 \\
3.3 \\
3.3\end{array}$ & $\begin{array}{l}5.5 \\
5.4 \\
5.4 \\
5.3 \\
5.0\end{array}$ & $\begin{array}{l}366 \\
364 \\
367 \\
370 \\
380\end{array}$ & $\begin{array}{l}0 \\
0 \\
0 \\
0 \\
0\end{array}$ & $\begin{array}{l}300 \\
299 \\
301 \\
303 \\
310\end{array}$ & $\begin{array}{l}800 \\
850 \\
800 \\
810 \\
820\end{array}$ & $\begin{array}{l}90 \\
91 \\
90 \\
86 \\
87\end{array}$ \\
\hline $\begin{array}{l}73 \\
74 \\
75\end{array}$ & $\begin{array}{l}220 \\
190 \\
220\end{array}$ & $\begin{array}{l}3.3 \\
2.9 \\
3.3\end{array}$ & $\begin{array}{l}5.2 \\
5.4 \\
5.3\end{array}$ & $\begin{array}{l}390 \\
370 \\
370\end{array}$ & $\begin{array}{l}0 \\
0 \\
0\end{array}$ & $\begin{array}{l}310 \\
300 \\
300\end{array}$ & $\begin{array}{l}800 \\
780 \\
780\end{array}$ & $\begin{array}{l}82 \\
83 \\
89\end{array}$ \\
\hline
\end{tabular}

NITRO- NITRO- PHOS-

GEN, GEN,AM- FHORUS,

MMONONIA MONIA + ORTHO, IRON, NESE, ORGANIC

DIS- ORGANIC DIS- DIS- DIS- DIS-

SOLVED DIS. SOLVED SOLVED SOLVED SOLVED

(MG/L (MG/L (MG/L $\quad(U G / L$ (UG/L (MG/L

$\begin{array}{lllll}\text { AS N) } & \text { AS N) } & \text { AS P) } & \text { AS } T E \text { ) } & \text { AS MN) }\end{array}$

$\begin{array}{rrrrrr}.06 & .53 & .02 & 150 & 2000 & -- \\ .05 & .68 & .00 & 1000 & 950 & -- \\ .14 & .57 & .05 & 240 & 1300 & -- \\ -- & -- & .05 & 40 & 100 & -- \\ .02 & .41 & .07 & 50 & 120 & -- \\ .04 & .24 & .03 & 40 & 110 & -- \\ .05 & .38 & .03 & 30 & 70 & -- \\ .06 & .25 & .04 & 20 & 30 & -- \\ .00 & .20 & .03 & 10 & 20 & -- \\ .01 & .18 & .06 & 30 & 20 & -- \\ .00 & .52 & .04 & 60 & 0 & -- \\ .00 & .34 & .04 & 40 & 5 & -- \\ .02 & .08 & .02 & 10 & 10 & -- \\ .00 & .17 & .06 & 20 & 0 & 17 \\ .00 & .16 & .07 & 30 & 10 & 1.6 \\ .00 & .21 & .03 & 30 & 10 & -- \\ .00 & .29 & .05 & 70 & 10 & -- \\ .00 & .32 & .05 & 100 & 10 & -- \\ .00 & .28 & .03 & 80 & 0 & -- \\ .01 & .82 & .11 & 170 & 20 & -- \\ .00 & .54 & .02 & 150 & 10 & -- \\ .00 & 1.5 & .04 & 100 & 10 & -\end{array}$


Table 5.--Water-level records and chemical [We11 number 19 on figure 2. Local we11 number is SB00506424CCA2.

\begin{tabular}{|c|c|c|c|c|c|c|c|c|}
\hline $\begin{array}{c}\text { DATE OF } \\
\text { ULR-L.EVEL } \\
\text { MEASUREMENT }\end{array}$ & $\begin{array}{l}\text { WATER } \\
\text { LEVEL } \\
\text { (FEET } \\
\text { EELOW } \\
\text { IAND } \\
\text { SURFACE) }\end{array}$ & $\begin{array}{c}\text { DATE } \\
\text { OF } \\
\text { SAMPLE }\end{array}$ & $\begin{array}{l}\text { SPE- } \\
\text { CIFIC } \\
\text { COH- } \\
\text { DUCT- } \\
\text { ANCE } \\
\text { (MICRO- } \\
\text { MHOS) }\end{array}$ & $\begin{array}{c}\text { PH } \\
\text { (UNITS) }\end{array}$ & $\begin{array}{l}\text { TFMPER- } \\
\text { ATURE } \\
\text { (DEG C) }\end{array}$ & $\begin{array}{l}\text { HARO- } \\
\text { NESS } \\
\text { (MG/L } \\
\text { AS } \\
\text { CACO3) }\end{array}$ & $\begin{array}{l}\text { MARD.- } \\
\text { NESS, } \\
\text { NONCAR- } \\
\text { BONATE } \\
\text { (MIG/L } \\
\text { CACOB) }\end{array}$ & $\begin{array}{l}\text { CALCIUM, } \\
\text { UIS- } \\
\text { SOLVED } \\
\text { (MGG,L } \\
\text { AS CA) }\end{array}$ \\
\hline $\begin{array}{l}74-04-04 \\
74-05-08 \\
74-06-14 \\
74-07-23 \\
74-08-27\end{array}$ & $\begin{array}{l}32.08 \\
32.38 \\
32.26 \\
32.65 \\
32.58\end{array}$ & $\begin{array}{l}74-04-04 \\
74-05-09 \\
74-06-14 \\
74-07-24 \\
74-03-28\end{array}$ & $\begin{array}{l}2430 \\
2450 \\
2440 \\
2450 \\
2430\end{array}$ & $\begin{array}{l}7.1 \\
7.3 \\
7.5 \\
7.4 \\
-.\end{array}$ & $\begin{array}{l}12.5 \\
13.5 \\
13.5 \\
14.5 \\
14.0\end{array}$ & $\begin{array}{l}990 \\
970 \\
990 \\
970 \\
990\end{array}$ & $\begin{array}{l}730 \\
650 \\
680 \\
660 \\
680\end{array}$ & $\begin{array}{l}240 \\
230 \\
240 \\
230 \\
240\end{array}$ \\
\hline $\begin{array}{l}74-10-03 \\
74-11-11 \\
74-12-05 \\
75-02-06 \\
75-05-14\end{array}$ & $\begin{array}{l}32.23 \\
32.08 \\
32.13 \\
32.35 \\
32.69\end{array}$ & $\begin{array}{l}74-10-03 \\
74-11-11 \\
74-12-05 \\
75-02-06 \\
75-05-14\end{array}$ & $\begin{array}{l}2420 \\
2400 \\
2600 \\
2600 \\
3100\end{array}$ & $\begin{array}{l}7 . \overline{2} \\
7.1 \\
7.1 \\
7.2\end{array}$ & $\begin{array}{l}13.0 \\
13.5 \\
13.0 \\
12.5 \\
13.5\end{array}$ & $\begin{array}{r}920 \\
940 \\
1000 \\
1000 \\
990\end{array}$ & $\begin{array}{l}600 \\
630 \\
690 \\
690 \\
680\end{array}$ & $\begin{array}{l}210 \\
230 \\
240 \\
240 \\
240\end{array}$ \\
\hline $\begin{array}{l}75-08-12 \\
75-12-01 \\
76-03-29 \\
76-06-29 \\
76-09-22\end{array}$ & $\begin{array}{l}32.83 \\
31.92 \\
32.38 \\
33.00 \\
32.32\end{array}$ & $\begin{array}{l}75-08-12 \\
75-12-02 \\
76-03-29 \\
76-06-29 \\
76-09-22\end{array}$ & $\begin{array}{l}3100 \\
2900 \\
2400 \\
2500 \\
2400\end{array}$ & $\begin{array}{l}7.7 \\
7.3 \\
7.7 \\
6.7 \\
7.3\end{array}$ & $\begin{array}{l}14.0 \\
13.5 \\
13.0 \\
13.5 \\
13.5\end{array}$ & $\begin{array}{r}1000 \\
960 \\
1000 \\
1000 \\
1000\end{array}$ & $\begin{array}{l}700 \\
650 \\
690 \\
680 \\
710\end{array}$ & $\begin{array}{l}240 \\
230 \\
250 \\
240 \\
250\end{array}$ \\
\hline $\begin{array}{l}76-12-08 \\
77-03-08 \\
77-06-15 \\
77.09-13 \\
77-12-06\end{array}$ & $\begin{array}{l}32.07 \\
32.41 \\
33.10 \\
33.18 \\
32.34\end{array}$ & $\begin{array}{l}76-12-08 \\
77-03-08 \\
77-06-15 \\
77-09-13 \\
77-12-06\end{array}$ & $\begin{array}{l}2400 \\
2600 \\
2500 \\
2600 \\
2600\end{array}$ & $\begin{array}{l}7.4 \\
7.5 \\
7.4 \\
7.4 \\
7.3\end{array}$ & $\begin{array}{l}13.0 \\
13.5 \\
13.5 \\
13.5 \\
12.5\end{array}$ & $\begin{array}{l}1000 \\
1000 \\
1000 \\
1100 \\
1100\end{array}$ & $\begin{array}{l}690 \\
680 \\
710 \\
110 \\
740\end{array}$ & $\begin{array}{l}240 \\
240 \\
250 \\
250 \\
250\end{array}$ \\
\hline $\begin{array}{l}78-03-09 \\
70-06-20\end{array}$ & $\begin{array}{l}32.44 \\
32.50\end{array}$ & $\begin{array}{l}78-03-09 \\
78-06-20\end{array}$ & $\begin{array}{l}2700 \\
2600\end{array}$ & $\begin{array}{l}7.3 \\
7.3\end{array}$ & $\begin{array}{l}13.0 \\
13.5\end{array}$ & $\begin{array}{l}1100 \\
1000\end{array}$ & $\begin{array}{r}1000 \\
690\end{array}$ & $\begin{array}{l}250 \\
230\end{array}$ \\
\hline $\begin{array}{c}\text { DATE OF } \\
\text { WMTER-LEVEL } \\
\text { MEASUREMENT }\end{array}$ & $\begin{array}{l}\text { WATER } \\
\text { LEVEL } \\
\text { (FEET } \\
\text { BELOW } \\
\text { LAND } \\
\text { SURFACE) }\end{array}$ & $\begin{array}{c}\text { DATE } \\
\text { OF } \\
\text { SAMPLE }\end{array}$ & $\begin{array}{l}\text { FLUO- } \\
\text { RIDE, } \\
\text { DIS- } \\
\text { SOLVED } \\
\text { (MG/L } \\
\text { AS F) }\end{array}$ & $\begin{array}{l}\text { SILICA, } \\
\text { DIS- } \\
\text { SOI.VED } \\
\text { (MG/L } \\
\text { AS } \\
\text { SID2) }\end{array}$ & $\begin{array}{l}\text { SOLIDS, } \\
\text { SUM OF } \\
\text { CONSTI- } \\
\text { TUENTS, } \\
\text { OIS- } \\
\text { SOLVED } \\
\text { (MG/L) }\end{array}$ & $\begin{array}{l}\text { NITRO- } \\
\text { GEN, } \\
\text { NITRATE } \\
\text { DIS- } \\
\text { SOI.VED } \\
\text { (MG/L } \\
\text { AS N) }\end{array}$ & $\begin{array}{l}\text { NITRO- } \\
\text { GEN, } \\
\text { NITRITE } \\
\text { DIS- } \\
\text { SOLVED } \\
\text { (MG/L } \\
\text { AS NI) }\end{array}$ & $\begin{array}{c}\text { NITRO- } \\
\text { GEN, } \\
\text { NO2+ +NO3 } \\
\text { DIS- } \\
\text { SOLVED } \\
\text { (MG/L } \\
\text { AS Nj }\end{array}$ \\
\hline $\begin{array}{l}74-04-04 \\
74-05-08 \\
74-06-14 \\
74-07-23 \\
74-08-27\end{array}$ & $\begin{array}{l}32.08 \\
32.38 \\
32.26 \\
32.65 \\
32.58\end{array}$ & $\begin{array}{l}74-04-04 \\
74-05-09 \\
74-06-14 \\
74-07-24 \\
74-08-28\end{array}$ & $\begin{array}{l}1.3 \\
1.3 \\
1.3 \\
1.3 \\
1.3\end{array}$ & $\begin{array}{l}19 \\
19 \\
19 \\
20 \\
20\end{array}$ & $\begin{array}{l}1870 \\
1790 \\
1760 \\
1810 \\
1810\end{array}$ & $\begin{array}{l}4.4 \\
5.7 \\
5.9 \\
-- \\
--\end{array}$ & $\begin{array}{l}.10 \\
.05 \\
.02 \\
.- \\
.02\end{array}$ & $\begin{array}{l}4.5 \\
5.7 \\
5.9 \\
5.8 \\
4.2\end{array}$ \\
\hline $\begin{array}{l}74-10-03 \\
74-11-11 \\
74-12-05 \\
75-02-06 \\
75-05-14\end{array}$ & $\begin{array}{l}32.23 \\
32.08 \\
32.13 \\
32.35 \\
32.69\end{array}$ & $\begin{array}{l}74-10-03 \\
74-11-11 \\
74-12-05 \\
75-02-06 \\
75-05-14\end{array}$ & $\begin{array}{l}1.3 \\
1.1 \\
1.3 \\
1.2 \\
1.3\end{array}$ & $\begin{array}{l}20 \\
19 \\
19 \\
20 \\
19\end{array}$ & $\begin{array}{l}1750 \\
1710 \\
1780 \\
1850 \\
1810\end{array}$ & $\begin{array}{l}6.0 \\
6.4 \\
5.8 \\
6.5 \\
5.8\end{array}$ & $\begin{array}{l}.01 \\
.00 \\
.01 \\
.01 \\
.02\end{array}$ & $\begin{array}{l}6.0 \\
6.4 \\
5.8 \\
6.5 \\
5.8\end{array}$ \\
\hline $\begin{array}{l}75-08-12 \\
75-12-01 \\
75-03-29 \\
75-06-29 \\
76-09-22\end{array}$ & $\begin{array}{l}32.83 \\
31.92 \\
32.38 \\
33.00 \\
32.32\end{array}$ & $\begin{array}{l}75-08-12 \\
75-12-02 \\
76-03-29 \\
75-06-29 \\
76-09-22\end{array}$ & $\begin{array}{l}1.2 \\
1.3 \\
1.2 \\
1.2 \\
1.3\end{array}$ & $\begin{array}{l}19 \\
19 \\
19 \\
18 \\
19\end{array}$ & $\begin{array}{l}1870 \\
1850 \\
1820 \\
1940 \\
1950\end{array}$ & $\begin{array}{l}6.3 \\
5.8 \\
7.1 \\
6.9 \\
7.5\end{array}$ & $\begin{array}{l}.02 \\
.02 \\
.01 \\
.01 \\
.01\end{array}$ & $\begin{array}{l}6.3 \\
5.8 \\
7.1 \\
6.9 \\
7.5\end{array}$ \\
\hline $\begin{array}{l}76-12-08 \\
77-03-03 \\
77-06-15 \\
77-03-13 \\
77-12-06\end{array}$ & $\begin{array}{l}32.07 \\
32.41 \\
33.10 \\
33.18 \\
32.31\end{array}$ & $\begin{array}{l}76-12-08 \\
77-03-08 \\
77-06-15 \\
77-09-13 \\
77-12-06\end{array}$ & $\begin{array}{l}1.3 \\
1.4 \\
1.4 \\
1.3 \\
1.3\end{array}$ & $\begin{array}{l}21 \\
20 \\
19 \\
20 \\
21\end{array}$ & $\begin{array}{l}1930 \\
1920 \\
1980 \\
1920 \\
2000\end{array}$ & $\begin{array}{l}9.3 \\
7.7 \\
7.2 \\
7.4 \\
8.2\end{array}$ & $\begin{array}{l}.01 \\
.01 \\
.00 \\
.00 \\
.01\end{array}$ & $\begin{array}{l}9.3 \\
7.0 \\
7.2 \\
7.4 \\
8.2\end{array}$ \\
\hline $\begin{array}{l}78-03-09 \\
78-06-20\end{array}$ & $\begin{array}{l}32.44 \\
32.50\end{array}$ & $\begin{array}{l}78-03-09 \\
78-06-20\end{array}$ & $\begin{array}{l}1.3 \\
1.2\end{array}$ & $\begin{array}{l}18 \\
16\end{array}$ & $\begin{array}{l}1820 \\
1970\end{array}$ & $\begin{array}{l}8.4 \\
7.7\end{array}$ & $\begin{array}{l}.00 \\
.01\end{array}$ & $\begin{array}{l}8.4 \\
7.7\end{array}$ \\
\hline
\end{tabular}


analyses of water from observation wells--Continued

Depth is $73 \mathrm{ft}$. Interval open to the aquifer is 69 to $73 \mathrm{ft}$ ]

\begin{tabular}{|c|c|c|c|c|c|c|c|c|}
\hline $\begin{array}{l}\text { HAGUE- } \\
\text { S!W" } \\
\text { DIS- } \\
\text { SOLVEI) } \\
\text { ( } M C_{6} / 1 \\
\text { AS MG) }\end{array}$ & $\begin{array}{l}\text { SODIUM, } \\
\text { DIS- } \\
\text { SOL_VED } \\
\text { (WG/L } \\
\text { AS NA })\end{array}$ & $\begin{array}{c}\text { SODIUP } \\
\text { AD- } \\
\text { SORF- } \\
\text { TIOHi } \\
\text { RATIO }\end{array}$ & $\begin{array}{l}\text { POTAS- } \\
\text { SIUH, } \\
\text { DIS- } \\
\text { SOLVED } \\
\text { (MG/L } \\
\text { AS K) }\end{array}$ & $\begin{array}{l}\text { BICAR- } \\
\text { POHATE } \\
\text { (MG/L } \\
\text { RS } \\
\text { HCO3) }\end{array}$ & $\begin{array}{c}\text { CAR- } \\
\text { BONATE } \\
\text { (MG/L } \\
\text { AS CO3) }\end{array}$ & $\begin{array}{l}\text { ALKA- } \\
\text { LINITY } \\
\text { (IIG/L } \\
\text { AS } \\
\text { CAC03) }\end{array}$ & $\begin{array}{c}\text { SULFATE, } \\
\text { NIS- } \\
\text { SOLVED } \\
\text { ("IG,L } \\
\text { AS SO4) }\end{array}$ & $\begin{array}{l}\text { CHLO- } \\
\text { RIDE, } \\
\text { DIS-- } \\
\text { SOLVED } \\
(\because I C / L \\
\text { AS }(\mathrm{CL})\end{array}$ \\
\hline $\begin{array}{l}96 \\
95 \\
95 \\
97 \\
96\end{array}$ & $\begin{array}{l}22.0 \\
200 \\
210 \\
220 \\
220\end{array}$ & $\begin{array}{l}3.0 \\
2.8 \\
2.9 \\
3.1 \\
3.0\end{array}$ & $\begin{array}{l}6.1 \\
5.4 \\
5.1 \\
5.1 \\
6.6\end{array}$ & $\begin{array}{l}328 \\
386 \\
380 \\
384 \\
383\end{array}$ & $\begin{array}{c}0 \\
0 \\
0 \\
-- \\
--\end{array}$ & $\begin{array}{l}269 \\
317 \\
312 \\
315 \\
314\end{array}$ & $\begin{array}{l}990 \\
900 \\
870 \\
900 \\
900\end{array}$ & $\begin{array}{l}110 \\
120 \\
110 \\
120 \\
120\end{array}$ \\
\hline $\begin{array}{l}95 \\
88 \\
90 \\
98 \\
94\end{array}$ & $\begin{array}{l}220 \\
220 \\
210 \\
210 \\
230\end{array}$ & $\begin{array}{l}3.2 \\
3.1 \\
2.9 \\
2.9 \\
3.2\end{array}$ & $\begin{array}{l}5.8 \\
3.4 \\
4.6 \\
5.3 \\
5.7\end{array}$ & $\begin{array}{l}381 \\
379 \\
383 \\
385 \\
374\end{array}$ & $\begin{array}{l}- \\
-- \\
-- \\
-0\end{array}$ & $\begin{array}{l}313 \\
311 \\
314 \\
316 \\
307\end{array}$ & $\begin{array}{l}870 \\
820 \\
880 \\
930 \\
890\end{array}$ & $\begin{array}{l}110 \\
110 \\
110 \\
130 \\
120\end{array}$ \\
\hline $\begin{array}{r}100 \\
93 \\
93 \\
99 \\
100\end{array}$ & $\begin{array}{l}220 \\
230 \\
210 \\
240 \\
230\end{array}$ & $\begin{array}{l}3.0 \\
3.2 \\
2.9 \\
3.3 \\
3.1\end{array}$ & $\begin{array}{l}5.7 \\
5.4 \\
5.6 \\
5.5 \\
5.6\end{array}$ & $\begin{array}{l}382 \\
379 \\
392 \\
304 \\
396\end{array}$ & $\begin{array}{l}0 \\
0 \\
0 \\
0 \\
0\end{array}$ & $\begin{array}{l}313 \\
311 \\
322 \\
323 \\
325\end{array}$ & $\begin{array}{r}940 \\
950 \\
910 \\
990 \\
1000\end{array}$ & $\begin{array}{l}130 \\
110 \\
110 \\
120 \\
120\end{array}$ \\
\hline $\begin{array}{r}100 \\
99 \\
100 \\
110 \\
110\end{array}$ & $\begin{array}{l}230 \\
230 \\
240 \\
220 \\
240\end{array}$ & $\begin{array}{l}3.1 \\
3.2 \\
3.2 \\
2.9 \\
3.2\end{array}$ & $\begin{array}{l}5.5 \\
5.5 \\
5.5 \\
5.2 \\
5.2\end{array}$ & $\begin{array}{l}395 \\
395 \\
400 \\
410 \\
410\end{array}$ & $\begin{array}{l}0 \\
0 \\
0 \\
0 \\
0\end{array}$ & $\begin{array}{l}324 \\
324 \\
328 \\
340 \\
340\end{array}$ & $\begin{array}{r}970 \\
970 \\
1000 \\
950 \\
1000\end{array}$ & $\begin{array}{l}130 \\
130 \\
130 \\
130 \\
130\end{array}$ \\
\hline $\begin{array}{l}110 \\
110\end{array}$ & $\begin{array}{l}220 \\
240\end{array}$ & $\begin{array}{l}2.9 \\
3.3\end{array}$ & $\begin{array}{l}5.8 \\
5.6\end{array}$ & $\begin{array}{r}70 \\
410\end{array}$ & $\begin{array}{l}0 \\
0\end{array}$ & $\begin{array}{r}57 \\
340\end{array}$ & $\begin{array}{l}1000 \\
1000\end{array}$ & $\begin{array}{l}140 \\
130\end{array}$ \\
\hline
\end{tabular}

\begin{tabular}{|c|c|c|c|c|c|}
\hline $\begin{array}{l}\text { NITPO- } \\
\text { GEN, } \\
\text { AMHONIA } \\
\text { DIS- } \\
\text { SOLVED } \\
\text { (MG/L } \\
\text { AS N) }\end{array}$ & $\begin{array}{l}\text { NITRO- } \\
\text { GLN,AM- } \\
\text { MOIIA + } \\
\text { ORGANIC } \\
\text { DIS. } \\
(M G / L \\
\text { ASN) }\end{array}$ & $\begin{array}{l}\text { PIOS- } \\
\text { PHORUS, } \\
\text { ORTIIO, } \\
\text { DIS- } \\
\text { SOLVED } \\
\text { (MG/L } \\
\text { AS P) }\end{array}$ & $\begin{array}{l}\text { IRON, } \\
\text { DIS- } \\
\text { SOLVED } \\
\text { (UG/L } \\
\text { AS FE) }\end{array}$ & $\begin{array}{l}\text { MANGA- } \\
\text { NESE, } \\
\text { DIS- } \\
\text { SOLVED } \\
\left(U G_{2} / L\right. \\
\text { AS MN) }\end{array}$ & $\begin{array}{l}\text { CARBON, } \\
\text { ORGANIC } \\
\text { DIS- } \\
\text { SOLVED } \\
\text { (MG/L } \\
\text { AS C) }\end{array}$ \\
\hline $\begin{array}{l}.05 \\
.18 \\
.11 \\
.01\end{array}$ & $\begin{array}{l}.89 \\
.71 \\
.61 \\
.74\end{array}$ & $\begin{array}{l}.07 \\
.02 \\
.06 \\
.05 \\
.09\end{array}$ & $\begin{array}{r}60 \\
130 \\
20 \\
30 \\
20\end{array}$ & $\begin{array}{r}2200 \\
1100 \\
390 \\
280 \\
200\end{array}$ & $\begin{array}{l}-- \\
-- \\
--\end{array}$ \\
\hline $\begin{array}{l}.03 \\
.04 \\
.04 \\
.00 \\
.01\end{array}$ & $\begin{array}{l}.3 \% \\
.42 \\
.25 \\
.36 \\
.33\end{array}$ & $\begin{array}{l}.03 \\
.03 \\
.05 \\
.03 \\
.07\end{array}$ & $\begin{array}{l}30 \\
40 \\
10 \\
10 \\
30\end{array}$ & $\begin{array}{r}150 \\
100 \\
60 \\
60 \\
50\end{array}$ & $\begin{array}{l}-- \\
-- \\
--\end{array}$ \\
\hline $\begin{array}{l}.00 \\
.00 \\
.02 \\
.00 \\
.00\end{array}$ & $\begin{array}{l}.41 \\
.56 \\
.36 \\
.38 \\
.34\end{array}$ & $\begin{array}{l}.06 \\
.06 \\
.03 \\
.06 \\
.05\end{array}$ & $\begin{array}{l}40 \\
30 \\
40 \\
20 \\
10\end{array}$ & $\begin{array}{l}20 \\
30 \\
20 \\
30 \\
30\end{array}$ & $\begin{array}{r}-- \\
-- \\
3.9 \\
2.9\end{array}$ \\
\hline $\begin{array}{l}.00 \\
.00 \\
.00 \\
.00\end{array}$ & $\begin{array}{l}.48 \\
.36 \\
.49 \\
.45 \\
.69\end{array}$ & $\begin{array}{l}.06 \\
.06 \\
.05 \\
.03 \\
.07\end{array}$ & $\begin{array}{l}30 \\
50 \\
90 \\
60 \\
70\end{array}$ & $\begin{array}{l}20 \\
20 \\
20 \\
20 \\
20\end{array}$ & $\begin{array}{l}-- \\
-- \\
--\end{array}$ \\
\hline $\begin{array}{l}.00 \\
.01\end{array}$ & $\frac{.71}{1.4}$ & $\begin{array}{l}.03 \\
.05\end{array}$ & $\begin{array}{l}40 \\
40\end{array}$ & $\begin{array}{l}20 \\
20\end{array}$ & $\bar{~}$ \\
\hline
\end{tabular}


Table 5.--Water-level records and chemical

[We11 number 20 on figure 2. Local we11 number is SB00506424CCA3.

$\begin{array}{ccc} & \\ & \text { WATEP } \\ \text { LATE OF } & \text { (FEET } \\ \text { WATER-LEVEL } & \text { BELOW } & \text { DAND } \\ \text { MEASUREMENT } & \text { SURFACE) } & \text { OF } \\ & \text { SAMPLE } \\ 74-04-04 & 32.33 & 74-04-04 \\ 74-05-08 & 32.55 & 74-05-09 \\ 74-06-14 & 32.57 & 74-06-14 \\ 74-07-23 & 32.90 & 74-07-24 \\ 74-08-27 & 32.80 & 74-08-28 \\ 74-10-03 & 32.45 & 74-10-03 \\ 74-11-11 & 32.27 & 74-11-11 \\ 74-12-05 & 32.43 & 74-12-05 \\ 75-02-06 & 32.58 & 75-02-06 \\ 75-05-14 & 32.89 & 75-05-14 \\ 75-08-12 & 33.28 & 75-08-12 \\ 75-12-02 & 32.15 & 75-12-02 \\ 76-03-29 & 33.14 & 76-03-29 \\ 76-05-29 & 33.01 & 76-06-29 \\ 76-09-22 & 32.57 & 76-09-22 \\ 76-12-08 & 32.29 & 76-12-08 \\ 77-03-08 & 32.57 & 77-03-08 \\ 77-06-15 & 33.2 n & 77-06-15 \\ 77-09-13 & 33.48 & 77-09-13 \\ 77-12-06 & 32.55 & 77-12-06 \\ 78-03-09 & 33.34 & 78-03-09 \\ 78-06-20 & 32.62 & 78-06-20 \\ & & \end{array}$
SPE-

CIFIC

CON-

DUCT-

ANCE

WATER-LEVEL

(MICRO-

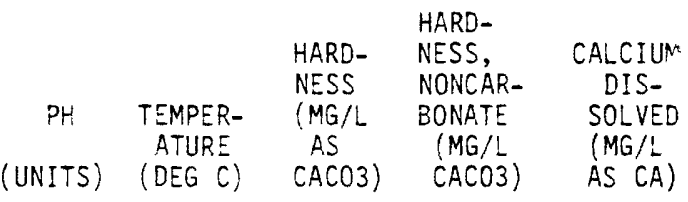

$74-04-04$

32.33

MHOS)

$\begin{array}{lccccc}2450 & 7.0 & 13.0 & 1000 & 620 & 250 \\ 2540 & 7.5 & 14.0 & 1000 & 69 C & 250 \\ 2600 & 7.5 & 13.5 & 1100 & 730 & 260 \\ 2630 & 7.5 & 14.0 & 1100 & 750 & 270 \\ 2670 & -- & 13.5 & 1100 & 790 & 270 \\ & & & & & \\ 2600 & -- & 13.5 & 1100 & 700 & 260 \\ 2550 & 7.3 & 13.0 & 920 & 580 & 210 \\ 2800 & 7.1 & 13.0 & 1100 & 780 & 270 \\ 2700 & 7.2 & 13.0 & 1000 & 690 & 250 \\ 3100 & 7.3 & 13.5 & 1000 & 670 & 240 \\ & & & & & \\ 3300 & 7.5 & 14.0 & 1000 & 670 & 230 \\ 3300 & 7.2 & 13.0 & 1100 & 720 & 270 \\ 2450 & 7.7 & 13.0 & 1000 & 640 & 240 \\ 2400 & 6.8 & 13.5 & 1100 & 680 & 240 \\ 2500 & 7.4 & 13.5 & 1100 & 710 & 250 \\ & & & & & \\ 2500 & 7.5 & 12.5 & 1100 & 730 & 250 \\ 2500 & 7.6 & 13.5 & 1100 & 690 & 250 \\ 2600 & 7.6 & 13.5 & 1100 & 770 & 260 \\ 2600 & 7.5 & 13.0 & 1100 & 700 & 250 \\ 2600 & 7.4 & 12.5 & 1100 & 730 & 250 \\ 2650 & 7.4 & 13.5 & 1100 & 1000 & 240 \\ 2600 & 7.4 & 13.0 & 1100 & 720 & 240 \\ & & \text { SOLIDS, } & \text { NITRO- } & \text { NITRO- } & \text { NITRO- } \\ \text { FLUO- } & \text { SILICA, } & \text { SUM OF } & \text { GEN, } & \text { GEN, } & \text { GEN, } \\ \text { RIDE, } & \text { DIS- } & \text { CONSTI- } & \text { NITRATE } & \text { NITRITE } & \text { NO2+NO3 } \\ \text { DIS- } & \text { SOLVED } & \text { TUENTS, } & \text { DIS- } & \text { DIS- } & \text { DIS- } \\ \text { SOLVED } & (M G / L & \text { DIS- } & \text { SOLVED } & \text { SOLVED } & \text { SOLVED } \\ \text { (MG/L } & \text { AS } & \text { SOLVED } & (\text { MG/L } & (\text { MG/L } & (\text { MG/L } \\ \text { AS F) } & \text { SIO2) } & (M G / L) & \text { AS N) } & \text { AS N) } & \text { AS N) } \\ & & & & & \end{array}$

MEASUREMENT SURFACE

$\begin{array}{lll}74-04-04 & 32.33 & 74-04-04 \\ 74-05-08 & 32.55 & 74-05-09 \\ 74-06-14 & 32.57 & 74-06-14 \\ 74-07-23 & 32.90 & 74-07-24 \\ 74-08-27 & 32.80 & 74-08-28 \\ & & \\ 74-10-03 & 32.45 & 74-10-03 \\ 74-11-11 & 32.27 & 74-11-11 \\ 74-12-05 & 32.43 & 74-12-05 \\ 75-02-06 & 32.58 & 75-02-06 \\ 75-05-14 & 32.89 & 75-05-14 \\ 75-08-12 & 33.28 & 75-08-12 \\ 75-12-02 & 32.15 & 75-12-02 \\ 76-03-29 & 33.14 & 76-03-29 \\ 76-06-29 & 33.01 & 76-06-29 \\ 76-09-22 & 32.57 & 76-09-22 \\ 76-12-08 & 32.29 & 76-12-08 \\ 77-03-08 & 32.57 & 77-03-08 \\ 77-06-15 & 33.20 & 77-06-15 \\ 77-09-13 & 33.48 & 77-09-13 \\ 77-12-06 & 32.55 & 77-12-06 \\ 78-03-09 & 33.34 & 78-03-09 \\ 78-06-20 & 32.62 & 78-06-20\end{array}$

\section{$1.8 \quad 21$}

$2.2 \quad 20$

$2.7 \quad 20$

$2.0 \quad 21$

$2.0 \quad 21$

1.921

$1.6 \quad 21$

1.820

1.821

$1.7 \quad 21$

1.720

$2.0 \quad 19$

$1.8 \quad 19$

$1.7 \quad 19$

1.720

1.720

$1.8 \quad 21$

$1.8 \quad 19$

1.921

1.921

$1.9 \quad 19$

$1.9 \quad 17$

1920
1920
1890
1980
2020
1950
1840
2000
1990
1840

$11^{4.5}$

12

12

$-$

11

13

12

8.6

1900

2120

2000

1990

9.6
9.3

10

11

2030

2000

1940

2050

1780

1950

\section{5}

12

12

19

$\begin{array}{ll}.17 & 4.7 \\ .06 & 11 \\ .02 & 12 \\ -0 & 13 \\ .01 & 13 \\ & \\ .00 & 11 \\ .00 & 13 \\ .00 & 12 \\ .00 & 11 \\ .00 & 8.6\end{array}$

.01

$$
9.6
$$

9.3 10

11

.0011

$.00 \quad 15$

$.01 \quad 12$

$.00 \quad 12$

$.00 \quad 14$

$.01 \quad 19$

$.00 \quad 15$

$.02 \quad 18$ 
analyses of water from observation wells--Continued

Depth is $38 \mathrm{ft}$. Interval open to the aquifer is 34 to $38 \mathrm{ft}$ ]

\begin{tabular}{|c|c|c|c|c|c|c|c|c|}
\hline $\begin{array}{l}\text { MAGNE- } \\
\text { SIUM, } \\
\text { DIS- } \\
\text { SOLVED } \\
\text { (MG/L } \\
\text { A.S MG) }\end{array}$ & $\begin{array}{l}\text { SODIUM, } \\
\text { DIS- } \\
\text { SOLVED } \\
\text { (MG/L } \\
\text { AS NA) }\end{array}$ & $\begin{array}{c}\text { SOOIUM } \\
\text { AL- } \\
\text { SORP- } \\
\text { T:OH } \\
\text { RATIO }\end{array}$ & $\begin{array}{l}\text { POTAS- } \\
\text { SIUM, } \\
\text { DIS- } \\
\text { SOLVED } \\
\text { (ME/L } \\
\text { AS K') }\end{array}$ & $\begin{array}{l}\text { BICAR- } \\
\text { BONATE } \\
\text { (MG/L } \\
\text { AS } \\
\text { HCO3) }\end{array}$ & $\begin{array}{l}\text { CAR- } \\
\text { BONATE } \\
\text { (MG/L } \\
\text { AS CO3) }\end{array}$ & $\begin{array}{l}\text { ALK.4- } \\
\text { LINITY } \\
\text { (MG/1- } \\
\text { A.S } \\
\text { CACO3) }\end{array}$ & $\begin{array}{c}\text { SLLLFATE, } \\
\text { DIS- } \\
\text { SOLVED } \\
\text { (MG/L } \\
\text { AS SO4) }\end{array}$ & $\begin{array}{l}\text { CHLO- } \\
\text { RIDE, } \\
\text { DIS- } \\
\text { SOLVED } \\
(M G / I- \\
\text { AS CL) }\end{array}$ \\
\hline $\begin{array}{r}96 \\
98 \\
100 \\
100 \\
110\end{array}$ & $\begin{array}{l}220 \\
210 \\
220 \\
230 \\
230\end{array}$ & $\begin{array}{l}3.0 \\
2.9 \\
2.9 \\
3.0 \\
3.0\end{array}$ & $\begin{array}{l}7.6 \\
6.8 \\
6.5 \\
7.5 \\
8.1\end{array}$ & $\begin{array}{l}484 \\
413 \\
400 \\
405 \\
410\end{array}$ & $\begin{array}{r}0 \\
0 \\
0 \\
-- \\
--\end{array}$ & $\begin{array}{l}397 \\
330 \\
328 \\
333 \\
336\end{array}$ & $\begin{array}{r}960 \\
970 \\
930 \\
980 \\
1000\end{array}$ & $\begin{array}{l}95 \\
110 \\
100 \\
110 \\
120\end{array}$ \\
\hline $\begin{array}{r}100 \\
96 \\
110 \\
100 \\
100\end{array}$ & $\begin{array}{l}230 \\
240 \\
230 \\
230 \\
230\end{array}$ & $\begin{array}{l}3.1 \\
3.4 \\
3.0 \\
3.1 \\
3.1\end{array}$ & $\begin{array}{l}7.3 \\
4.3 \\
5.9 \\
6.8 \\
6.8\end{array}$ & $\begin{array}{l}446 \\
414 \\
419 \\
428 \\
412\end{array}$ & $\begin{array}{c}-- \\
-- \\
--\end{array}$ & $\begin{array}{l}366 \\
340 \\
344 \\
351 \\
338\end{array}$ & $\begin{array}{l}950 \\
910 \\
990 \\
990 \\
880\end{array}$ & $\begin{array}{l}110 \\
100 \\
110 \\
130 \\
120\end{array}$ \\
\hline $\begin{array}{l}110 \\
100 \\
100 \\
110 \\
110\end{array}$ & $\begin{array}{l}230 \\
260 \\
230 \\
250 \\
230\end{array}$ & $\begin{array}{l}3.1 \\
3.4 \\
3.1 \\
3.4 \\
3.1\end{array}$ & $\begin{array}{l}7.2 \\
7.2 \\
7.5 \\
7.5 \\
7.5\end{array}$ & $\begin{array}{l}430 \\
448 \\
450 \\
454 \\
450\end{array}$ & $\begin{array}{l}0 \\
0 \\
0 \\
0 \\
0\end{array}$ & $\begin{array}{l}353 \\
367 \\
369 \\
372 \\
369\end{array}$ & $\begin{array}{r}940 \\
1100 \\
950 \\
1000 \\
1000\end{array}$ & $\begin{array}{r}110 \\
100 \\
98 \\
98 \\
96\end{array}$ \\
\hline $\begin{array}{l}120 \\
110 \\
120 \\
110 \\
120\end{array}$ & $\begin{array}{l}230 \\
230 \\
230 \\
210 \\
240\end{array}$ & $\begin{array}{l}3.0 \\
3.1 \\
3.0 \\
2.8 \\
3.1\end{array}$ & $\begin{array}{l}7.6 \\
7.6 \\
7.8 \\
7.2 \\
6.8\end{array}$ & $\begin{array}{l}471 \\
470 \\
460 \\
460 \\
470\end{array}$ & $\begin{array}{l}0 \\
0 \\
0 \\
0 \\
0\end{array}$ & $\begin{array}{l}386 \\
385 \\
377 \\
380 \\
390\end{array}$ & $\begin{array}{r}1000 \\
980 \\
980 \\
950 \\
1000\end{array}$ & $\begin{array}{l}100 \\
110 \\
100 \\
100 \\
100\end{array}$ \\
\hline $\begin{array}{l}120 \\
120\end{array}$ & $\begin{array}{l}210 \\
230\end{array}$ & $\begin{array}{l}2.8 \\
3.0\end{array}$ & $\begin{array}{l}8.0 \\
7.7\end{array}$ & $\begin{array}{l}120 \\
450\end{array}$ & $\begin{array}{l}0 \\
0\end{array}$ & $\begin{array}{r}98 \\
370\end{array}$ & $\begin{array}{l}960 \\
920\end{array}$ & $\begin{array}{l}100 \\
110\end{array}$ \\
\hline $\begin{array}{l}\text { PITRO- } \\
\text { EENI, G } \\
\text { MMMONIA } \\
\text { DIS- O } \\
\text { SOLVED } \\
\text { (ME/L } \\
\text { ISN) }\end{array}$ & $\begin{array}{l}\text { NITRO- } \\
\text { GEN,AM- } \\
\text { MONIA + } \\
\text { ORGANIC } \\
\text { DIS. } \\
\text { (MG/L. } \\
\text { AS N) }\end{array}$ & $\begin{array}{l}\text { PHOS- } \\
\text { PHORUS, } \\
\text { ORTHO, } \\
\text { DIS- } \\
\text { SOLVED } \\
\text { (MG/L } \\
\text { AS P) }\end{array}$ & $\begin{array}{l}\text { IRON, } \\
\text { DIS- } \\
\text { SOLVED } \\
\text { (UG/L } \\
\text { AS FE) }\end{array}$ & $\begin{array}{l}\text { MANGA- } \\
\text { NESE, } \\
\text { DIS- } \\
\text { SOLVED } \\
\text { (UG/L } \\
\text { AS MN) }\end{array}$ & $\begin{array}{l}\text { CARBON, } \\
\text { ORGANIC } \\
\text { DIS- } \\
\text { SOLVED } \\
(M G / L \\
\text { AS C) }\end{array}$ & & & \\
\hline $\begin{array}{l}.09 \\
.12 \\
.05 \\
-. \\
.03\end{array}$ & $\begin{array}{r}1.1 \\
.56 \\
.75 \\
.- \\
.46\end{array}$ & $\begin{array}{l}.04 \\
.01 \\
.06 \\
.07 \\
.12\end{array}$ & $\begin{array}{l}50 \\
50 \\
20 \\
20 \\
40\end{array}$ & $\begin{array}{r}4900 \\
210 \\
40 \\
10 \\
30\end{array}$ & $\begin{array}{l}-- \\
\cdots \\
\cdots \\
--\end{array}$ & & & \\
\hline $\begin{array}{l}1.9 \\
.01 \\
.04 \\
.01 \\
.01\end{array}$ & $\begin{array}{l}.30 \\
.48 \\
.37 \\
.45 \\
.40\end{array}$ & $\begin{array}{l}.05 \\
.04 \\
.05 \\
.04 \\
.07\end{array}$ & $\begin{array}{r}50 \\
30 \\
10 \\
0 \\
30\end{array}$ & $\begin{array}{r}30 \\
0 \\
10 \\
20 \\
10\end{array}$ & $\begin{array}{l}-- \\
-- \\
-- \\
--\end{array}$ & & & \\
\hline $\begin{array}{l}.00 \\
.00 \\
.02 \\
.00 \\
.00\end{array}$ & $\begin{array}{l}.64 \\
.91 \\
.47 \\
.26 \\
.52\end{array}$ & $\begin{array}{l}.07 \\
.08 \\
.06 \\
.08 \\
.06\end{array}$ & $\begin{array}{l}20 \\
30 \\
10 \\
20 \\
10\end{array}$ & $\begin{array}{r}0 \\
5 \\
0 \\
0 \\
10\end{array}$ & $\begin{array}{l}-- \\
-- \\
-- \\
4.0 \\
3.7\end{array}$ & & & \\
\hline $\begin{array}{l}.08 \\
.00 \\
.00 \\
.00 \\
.01\end{array}$ & $\begin{array}{l}.82 \\
.72 \\
.33 \\
.54 \\
2.0\end{array}$ & $\begin{array}{l}.05 \\
.07 \\
.05 \\
.04 \\
.06\end{array}$ & $\begin{array}{r}20 \\
40 \\
30 \\
30 \\
150\end{array}$ & $\begin{array}{r}0 \\
10 \\
10 \\
10 \\
4\end{array}$ & $\begin{array}{l}-- \\
-- \\
-- \\
--\end{array}$ & v & & \\
\hline $\begin{array}{l}.00 \\
.01\end{array}$ & $\begin{array}{l}.95 \\
1.8\end{array}$ & $\begin{array}{l}.04 \\
.04\end{array}$ & $\begin{array}{l}20 \\
10\end{array}$ & $\begin{array}{l}0 \\
5\end{array}$ & -- & & & \\
\hline
\end{tabular}


Table 5.--Water-level records and chemical [We11 number 23 on figure 2. Local well number is SB00506425ACB1.

\begin{tabular}{|c|c|c|c|c|c|c|c|c|}
\hline $\begin{array}{l}\text { DATE OF } \\
\text { WATER-LEVEL } \\
\text { MEASUREMENT }\end{array}$ & $\begin{array}{l}\text { WATER } \\
\text { LEVEL } \\
\text { (FEET } \\
\text { BELOW } \\
\text { LAND } \\
\text { SURFACE) }\end{array}$ & $\begin{array}{c}\text { DATE } \\
\text { OF } \\
\text { SAMPLE }\end{array}$ & $\begin{array}{l}\text { SPE- } \\
\text { CIFIC } \\
\text { CON- } \\
\text { DUCT- } \\
\text { ANCE } \\
\text { (MICRO- } \\
\text { MHOS) }\end{array}$ & $\begin{array}{c}\mathrm{PH} \\
\text { (UNITS) }\end{array}$ & $\begin{array}{l}\text { TEMPER- } \\
\text { ATURE } \\
\text { (DEG C) }\end{array}$ & $\begin{array}{l}\text { HARD- } \\
\text { NESS } \\
\text { (MG/L } \\
\text { AS } \\
\text { CACO3) }\end{array}$ & $\begin{array}{l}\text { HARD- } \\
\text { NESS, } \\
\text { NONCAR- } \\
\text { BONATE } \\
\text { (MG/L } \\
\text { CACO3) }\end{array}$ & $\begin{array}{l}\text { CALCIUM, } \\
\text { DIS- } \\
\text { SOLVED } \\
\text { (MG/L } \\
\text { AS CA) }\end{array}$ \\
\hline $\begin{array}{l}74-04-03 \\
74-05-08 \\
74-06-13 \\
74-07-23 \\
74-08-27\end{array}$ & $\begin{array}{l}34.14 \\
34.18 \\
34.44 \\
34.69 \\
34.55\end{array}$ & $\begin{array}{l}74-04-03 \\
74-05-08 \\
74-06-13 \\
74-07-23 \\
74-08-27\end{array}$ & $\begin{array}{l}2730 \\
2470 \\
2340 \\
2470 \\
2700\end{array}$ & $\begin{array}{l}6.5 \\
6.9 \\
7.1 \\
7.3 \\
7.2\end{array}$ & $\begin{array}{l}11.5 \\
13.5 \\
13.5 \\
15.0 \\
14.5\end{array}$ & $\begin{array}{r}1100 \\
920 \\
830 \\
830 \\
920\end{array}$ & $\begin{array}{l}600 \\
520 \\
500 \\
520 \\
600\end{array}$ & $\begin{array}{l}290 \\
230 \\
210 \\
210 \\
220\end{array}$ \\
\hline $\begin{array}{l}74-10-03 \\
74-11-11 \\
74-12-05 \\
75-02-06 \\
75-05-14\end{array}$ & $\begin{array}{l}33.58 \\
33.50 \\
33.66 \\
33.98 \\
34.52\end{array}$ & $\begin{array}{l}74-10-03 \\
74-11-11 \\
74-12-05 \\
75-02-06 \\
75-05-14\end{array}$ & $\begin{array}{l}2920 \\
3000 \\
3200 \\
3100 \\
3400\end{array}$ & $\begin{array}{l}-. \overline{7} \\
6.9 \\
7.0 \\
7.2\end{array}$ & $\begin{array}{l}13.5 \\
12.5 \\
12.0 \\
12.0 \\
13.5\end{array}$ & $\begin{array}{r}960 \\
1000 \\
1100 \\
970 \\
940\end{array}$ & $\begin{array}{l}600 \\
660 \\
740 \\
640 \\
600\end{array}$ & $\begin{array}{l}230 \\
250 \\
260 \\
230 \\
230\end{array}$ \\
\hline $\begin{array}{l}75-08-11 \\
75-12-01 \\
76-03-29 \\
76-06-29 \\
76-09-22\end{array}$ & $\begin{array}{l}34.16 \\
33.69 \\
34.12 \\
34.76 \\
34.35\end{array}$ & $\begin{array}{l}75-08-11 \\
75-12-01 \\
76-03-29 \\
76-06-29 \\
76-09-22\end{array}$ & $\begin{array}{l}2900 \\
3400 \\
2600 \\
2400 \\
2200\end{array}$ & $\begin{array}{l}7.2 \\
7.4 \\
7.7 \\
6.7 \\
7.2\end{array}$ & $\begin{array}{l}13.5 \\
12.5 \\
12.0 \\
14.0 \\
13.5\end{array}$ & $\begin{array}{r}800 \\
1000 \\
920 \\
760 \\
790\end{array}$ & $\begin{array}{l}520 \\
670 \\
580 \\
470 \\
510\end{array}$ & $\begin{array}{l}200 \\
250 \\
220 \\
190 \\
200\end{array}$ \\
\hline $\begin{array}{l}76-12-08 \\
77-03-08 \\
77-06-15 \\
77-09-13 \\
77-12-06\end{array}$ & $\begin{array}{l}33.83 \\
34.27 \\
34.88 \\
34.94 \\
34.09\end{array}$ & $\begin{array}{l}76-12-08 \\
77-03-08 \\
77-06-15 \\
77-09-13 \\
77-12-06\end{array}$ & $\begin{array}{l}2800 \\
2700 \\
2400 \\
2300 \\
2200\end{array}$ & $\begin{array}{l}7.3 \\
7.6 \\
7.3 \\
7.5 \\
7.3\end{array}$ & $\begin{array}{l}13.0 \\
13.0 \\
14.0 \\
13.0 \\
11.5\end{array}$ & $\begin{array}{r}1100 \\
940 \\
840 \\
850 \\
840\end{array}$ & $\begin{array}{l}710 \\
620 \\
540 \\
540 \\
540\end{array}$ & $\begin{array}{l}260 \\
230 \\
210 \\
210 \\
210\end{array}$ \\
\hline $\begin{array}{l}78-03-09 \\
78-06-20\end{array}$ & $\begin{array}{l}34.32 \\
34.37\end{array}$ & $\begin{array}{l}78-03-09 \\
78-06-20\end{array}$ & $\begin{array}{l}3500 \\
2400\end{array}$ & $\begin{array}{l}7.2 \\
7.0\end{array}$ & $\begin{array}{l}12.5 \\
13.5\end{array}$ & $\begin{array}{r}1300 \\
860\end{array}$ & $\begin{array}{l}900 \\
560\end{array}$ & $\begin{array}{l}300 \\
210\end{array}$ \\
\hline $\begin{array}{l}\text { DATE OF } \\
\text { WATER-LEVEL } \\
\text { MEASUREMENT }\end{array}$ & $\begin{array}{l}\text { WATER } \\
\text { LEVEL } \\
\text { (FEET } \\
\text { BELOW } \\
\text { LAND } \\
\text { SURFACE) }\end{array}$ & $\begin{array}{c}\text { DATE } \\
\text { OF } \\
\text { SAMPLE }\end{array}$ & $\begin{array}{l}\text { FLUO- } \\
\text { RIDE, } \\
\text { DIS- } \\
\text { SOLVED } \\
\text { (MG/L } \\
\text { AS F) }\end{array}$ & $\begin{array}{l}\text { SILICA, } \\
\text { DIS- } \\
\text { SOLVED } \\
\text { (MG/L } \\
\text { AS } \\
\text { SI02) }\end{array}$ & $\begin{array}{c}\text { SOLIDS, } \\
\text { SUM OF } \\
\text { CONSTI- } \\
\text { TUENTS, } \\
\text { DIS- } \\
\text { SOLVED } \\
\text { (MG/L) }\end{array}$ & $\begin{array}{c}\text { NITRO- } \\
\text { GEN, } \\
\text { NITRATE } \\
\text { DIS- } \\
\text { SOLVED } \\
(M G / L \\
\text { AS N) }\end{array}$ & $\begin{array}{c}\text { NITRO- } \\
\text { GEN, } \\
\text { NITRITE } \\
\text { DIS- } \\
\text { SOLVED } \\
\text { (MG/L } \\
\text { AS N) }\end{array}$ & $\begin{array}{c}\text { NITRO- } \\
\text { GEN, } \\
\text { NO2+NO3 } \\
\text { DIS- } \\
\text { SOLVED } \\
\text { (MG/L } \\
\text { AS N) }\end{array}$ \\
\hline $\begin{array}{l}74-04-03 \\
74-05-08 \\
74-06-13 \\
74-07-23 \\
74-08-27\end{array}$ & $\begin{array}{l}34.14 \\
34.18 \\
34.44 \\
34.69 \\
34.55\end{array}$ & $\begin{array}{l}74-04-03 \\
74-05-08 \\
74-06-13 \\
74-07-23 \\
74-08-27\end{array}$ & $\begin{array}{l}1.0 \\
1.2 \\
1.1 \\
1.3 \\
2.2\end{array}$ & $\begin{array}{l}22 \\
21 \\
19 \\
19 \\
24\end{array}$ & $\begin{array}{l}2010 \\
1820 \\
1700 \\
1790 \\
1920\end{array}$ & $\begin{array}{l}.44 \\
1.7 \\
2.2 \\
2 . \overline{9}\end{array}$ & $\begin{array}{l}.66 \\
.03 \\
.02 \\
.- \\
.00\end{array}$ & $\begin{array}{l}1.1 \\
1.7 \\
2.2 \\
3.8 \\
2.9\end{array}$ \\
\hline $\begin{array}{l}74-10-03 \\
74-11-11 \\
74-12-05 \\
75-02-06 \\
75-05-14\end{array}$ & $\begin{array}{l}33.58 \\
33.50 \\
33.66 \\
33.98 \\
34.52\end{array}$ & $\begin{array}{l}74-10-03 \\
74-11-11 \\
74-12-05 \\
75-02-06 \\
75-05-14\end{array}$ & $\begin{array}{l}1.4 \\
1.2 \\
1.4 \\
1.4 \\
1.4\end{array}$ & $\begin{array}{l}20 \\
20 \\
19 \\
20 \\
16\end{array}$ & $\begin{array}{l}2220 \\
2260 \\
2280 \\
1860 \\
2050\end{array}$ & $\begin{array}{l}4.8 \\
5.9 \\
6.1 \\
3.4 \\
.48\end{array}$ & $\begin{array}{l}.30 \\
.25 \\
.35 \\
.03 \\
.00\end{array}$ & $\begin{array}{l}5.1 \\
6.1 \\
6.4 \\
3.4 \\
.48\end{array}$ \\
\hline $\begin{array}{l}75-08-11 \\
75-12-01 \\
76-03-29 \\
76-06-29 \\
76-09-22\end{array}$ & $\begin{array}{l}34.16 \\
33.69 \\
34.12 \\
34.76 \\
34.35\end{array}$ & $\begin{array}{l}75-08-11 \\
75-12-01 \\
76-03-29 \\
76-06-29 \\
76-09-22\end{array}$ & $\begin{array}{l}1.1 \\
1.3 \\
1.2 \\
1.3 \\
1.3\end{array}$ & $\begin{array}{l}17 \\
16 \\
15 \\
16 \\
16\end{array}$ & $\begin{array}{l}1740 \\
2320 \\
1920 \\
1720 \\
1750\end{array}$ & $\begin{array}{l}3.7 \\
4.2 \\
1.9 \\
4.0 \\
4.2\end{array}$ & $\begin{array}{l}.08 \\
.01 \\
.01 \\
.05 \\
.02\end{array}$ & $\begin{array}{l}3.8 \\
4.2 \\
1.9 \\
4.0 \\
4.2\end{array}$ \\
\hline $\begin{array}{l}76-12-08 \\
77-03-08 \\
77-06-15 \\
77-09-13 \\
77-12-06\end{array}$ & $\begin{array}{l}33.83 \\
34.27 \\
34.88 \\
34.94 \\
34.09\end{array}$ & $\begin{array}{l}76-12-08 \\
77-03-08 \\
77-06-15 \\
77-09-13 \\
77-12-06\end{array}$ & $\begin{array}{l}1.3 \\
1.3 \\
1.3 \\
1.2 \\
1.3\end{array}$ & $\begin{array}{l}19 \\
18 \\
16 \\
17 \\
16\end{array}$ & $\begin{array}{l}2280 \\
2050 \\
1740 \\
1860 \\
1770\end{array}$ & $\begin{array}{l}11 \\
5.3 \\
3.7 \\
3.7 \\
2.1\end{array}$ & $\begin{array}{l}.01 \\
.00 \\
.01 \\
.01 \\
.01\end{array}$ & $\begin{array}{l}11 \\
5.3 \\
3.7 \\
3.7 \\
2.1\end{array}$ \\
\hline $\begin{array}{l}78-03-09 \\
78-06-20\end{array}$ & $\begin{array}{l}34.32 \\
34.37\end{array}$ & $\begin{array}{l}78-03-09 \\
78-06-20\end{array}$ & $\begin{array}{l}1.5 \\
1.1\end{array}$ & $\begin{array}{l}19 \\
13\end{array}$ & $\begin{array}{l}2760 \\
1760\end{array}$ & $\begin{array}{l}8.6 \\
1.9\end{array}$ & $\begin{array}{l}.00 \\
.03\end{array}$ & $\begin{array}{l}8.6 \\
1.9\end{array}$ \\
\hline
\end{tabular}


analyses of water from observation wells--Continued

Depth is $99 \mathrm{ft}$. Interval open to the aquifer is 94 to $98 \mathrm{ft}$ ]

\begin{tabular}{|c|c|c|c|c|c|c|c|c|}
\hline $\begin{array}{l}\text { MAGNE- } \\
\text { SIUM, } \\
\text { DIS- } \\
\text { SOLVED } \\
\text { (MG/L } \\
\text { AS MG) }\end{array}$ & $\begin{array}{l}\text { SODIUM, } \\
\text { DIS- } \\
\text { SOLVED } \\
\text { (MG/L } \\
\text { AS NA) }\end{array}$ & $\begin{array}{l}\text { SODIUM } \\
\text { AD- } \\
\text { SORP- } \\
\text { TION } \\
\text { RATIO }\end{array}$ & $\begin{array}{l}\text { POTAS- } \\
\text { SIUM, } \\
\text { DIS- } \\
\text { SOLVED } \\
\text { (MG/L } \\
\text { AS K) }\end{array}$ & $\begin{array}{l}\text { BICAR- } \\
\text { BONATE } \\
\text { (MG/L } \\
\text { AS } \\
\text { HCO3) }\end{array}$ & $\begin{array}{c}\text { CAR- } \\
\text { BONATE } \\
\text { (MG/L } \\
\text { AS CO3) }\end{array}$ & $\begin{array}{l}\text { ALKA- } \\
\text { LINITY } \\
\text { (MG/L } \\
\text { AS } \\
\text { CACO3) }\end{array}$ & $\begin{array}{l}\text { SULFATE, } \\
\text { DIS- } \\
\text { SOLVED } \\
\text { (MG/L } \\
\text { AS SO4) }\end{array}$ & $\begin{array}{l}\text { CHLO- } \\
\text { RIDE, } \\
\text { DIS- } \\
\text { SOLVED } \\
\text { (MG/L } \\
\text { AS CL) }\end{array}$ \\
\hline $\begin{array}{r}100 \\
83 \\
73 \\
75 \\
90\end{array}$ & $\begin{array}{l}250 \\
250 \\
240 \\
260 \\
290\end{array}$ & $\begin{array}{l}3.2 \\
3.6 \\
3.6 \\
3.9 \\
4.2\end{array}$ & $\begin{array}{l}6.8 \\
5.0 \\
4.8 \\
4.3 \\
7.1\end{array}$ & $\begin{array}{l}656 \\
481 \\
393 \\
380 \\
388\end{array}$ & $\begin{array}{c}0 \\
0 \\
0 \\
-- \\
--\end{array}$ & $\begin{array}{l}538 \\
395 \\
322 \\
312 \\
318\end{array}$ & $\begin{array}{r}910 \\
900 \\
880 \\
940 \\
1000\end{array}$ & $\begin{array}{l}94 \\
77 \\
66 \\
77 \\
82\end{array}$ \\
\hline $\begin{array}{r}93 \\
94 \\
110 \\
97 \\
88\end{array}$ & $\begin{array}{l}340 \\
350 \\
340 \\
230 \\
320\end{array}$ & $\begin{array}{l}4.8 \\
4.8 \\
4.5 \\
3.2 \\
4.6\end{array}$ & $\begin{array}{l}6.8 \\
4.0 \\
5.4 \\
6.7 \\
5.1\end{array}$ & $\begin{array}{l}435 \\
434 \\
441 \\
410 \\
412\end{array}$ & $\begin{array}{l}-- \\
\ddot{-} \\
\ddot{0}\end{array}$ & $\begin{array}{l}357 \\
356 \\
362 \\
336 \\
338\end{array}$ & $\begin{array}{r}1200 \\
1200 \\
1200 \\
940 \\
1100\end{array}$ & $\begin{array}{r}91 \\
95 \\
95 \\
120 \\
81\end{array}$ \\
\hline $\begin{array}{l}74 \\
94 \\
91 \\
70 \\
71\end{array}$ & $\begin{array}{l}260 \\
340 \\
300 \\
270 \\
250\end{array}$ & $\begin{array}{l}4.0 \\
4.7 \\
4.3 \\
4.3 \\
3.9\end{array}$ & $\begin{array}{l}5.9 \\
6.8 \\
6.6 \\
5.5 \\
5.6\end{array}$ & $\begin{array}{l}351 \\
416 \\
414 \\
353 \\
347\end{array}$ & $\begin{array}{l}0 \\
0 \\
0 \\
0 \\
0\end{array}$ & $\begin{array}{l}288 \\
341 \\
340 \\
290 \\
285\end{array}$ & $\begin{array}{r}920 \\
1300 \\
1000 \\
910 \\
950\end{array}$ & $\begin{array}{l}69 \\
85 \\
77 \\
70 \\
67\end{array}$ \\
\hline $\begin{array}{l}99 \\
88 \\
76 \\
79 \\
77\end{array}$ & $\begin{array}{l}340 \\
300 \\
260 \\
270 \\
250\end{array}$ & $\begin{array}{l}4.6 \\
4.3 \\
3.9 \\
4.0 \\
3.8\end{array}$ & $\begin{array}{l}6.5 \\
6.0 \\
5.3 \\
5.2 \\
5.7\end{array}$ & $\begin{array}{l}428 \\
390 \\
360 \\
380 \\
370\end{array}$ & $\begin{array}{l}0 \\
0 \\
0 \\
0 \\
0\end{array}$ & $\begin{array}{l}351 \\
320 \\
300 \\
310 \\
300\end{array}$ & $\begin{array}{r}1200 \\
1100 \\
910 \\
1000 \\
950\end{array}$ & $\begin{array}{l}97 \\
92 \\
68 \\
77 \\
71\end{array}$ \\
\hline $\begin{array}{r}130 \\
82\end{array}$ & $\begin{array}{l}410 \\
260\end{array}$ & $\begin{array}{l}5.0 \\
3.9\end{array}$ & $\begin{array}{l}8.0 \\
6.0\end{array}$ & $\begin{array}{l}470 \\
370\end{array}$ & $\begin{array}{l}0 \\
0\end{array}$ & $\begin{array}{l}390 \\
300\end{array}$ & $\begin{array}{r}1500 \\
920\end{array}$ & $\begin{array}{r}120 \\
78\end{array}$ \\
\hline
\end{tabular}

\begin{tabular}{|c|c|c|c|c|c|}
\hline $\begin{array}{c}\text { NITRO- } \\
\text { GEN, } \\
\text { AMMONIA } \\
\text { DIS- } \\
\text { SOLVED } \\
\text { (MG/L } \\
\text { AS N) }\end{array}$ & $\begin{array}{l}\text { NITRO- } \\
\text { GEN, AM- } \\
\text { MONIA + } \\
\text { ORGANIC } \\
\text { DIS. } \\
\text { (MG/L } \\
\text { AS N) }\end{array}$ & $\begin{array}{c}\text { PHOS- } \\
\text { PHORUS, } \\
\text { ORTHO, } \\
\text { DIS- } \\
\text { SOLVED } \\
\text { (MG/L } \\
\text { AS P) }\end{array}$ & $\begin{array}{l}\text { IRON, } \\
\text { DIS- } \\
\text { SOLVED } \\
\text { (UG/L } \\
\text { AS FE) }\end{array}$ & $\begin{array}{l}\text { MANGA- } \\
\text { NESE, } \\
\text { DIS- } \\
\text { SOLVED } \\
\text { (UG/L } \\
\text { AS MN) }\end{array}$ & $\begin{array}{l}\text { CARBON, } \\
\text { ORGANIC } \\
\text { DIS- } \\
\text { SOLVED } \\
\text { (MG/L } \\
\text { AS C) }\end{array}$ \\
\hline $\begin{array}{l}.17 \\
.17 \\
.34 \\
-.- \\
.15\end{array}$ & $\begin{array}{l}.64 \\
.71 \\
.81 \\
.44\end{array}$ & $\begin{array}{l}.02 \\
.00 \\
.04 \\
.04 \\
.05\end{array}$ & $\begin{array}{r}3300 \\
5300 \\
1500 \\
230 \\
310\end{array}$ & $\begin{array}{r}4900 \\
2700 \\
1500 \\
950 \\
810\end{array}$ & $\begin{array}{l}-- \\
-- \\
-- \\
--\end{array}$ \\
\hline $\begin{array}{l}.14 \\
.08 \\
.08 \\
.01 \\
.02\end{array}$ & $\begin{array}{l}.57 \\
.71 \\
.49 \\
.59 \\
.21\end{array}$ & $\begin{array}{l}.02 \\
.01 \\
.02 \\
.01 \\
.04\end{array}$ & $\begin{array}{l}60 \\
40 \\
30 \\
10 \\
60\end{array}$ & $\begin{array}{r}490 \\
300 \\
150 \\
10 \\
160\end{array}$ & $\begin{array}{l}-- \\
-- \\
-- \\
--\end{array}$ \\
\hline $\begin{array}{l}.00 \\
.08 \\
.04 \\
.00 \\
.00\end{array}$ & $\begin{array}{l}.21 \\
.94 \\
.30 \\
.25 \\
.23\end{array}$ & $\begin{array}{l}.02 \\
.02 \\
.00 \\
.04 \\
.01\end{array}$ & $\begin{array}{r}20 \\
130 \\
230 \\
100 \\
90\end{array}$ & $\begin{array}{r}110 \\
50 \\
50 \\
70 \\
50\end{array}$ & $\begin{array}{l}-- \\
-- \\
-- \\
2.2 \\
1.2\end{array}$ \\
\hline $\begin{array}{l}.00 \\
.02 \\
.05 \\
.01 \\
.00\end{array}$ & $\begin{array}{l}.84 \\
.71 \\
.33 \\
.39 \\
2.0\end{array}$ & $\begin{array}{l}.01 \\
.01 \\
.03 \\
.03 \\
.01\end{array}$ & $\begin{array}{r}60 \\
110 \\
100 \\
90 \\
160\end{array}$ & $\begin{array}{l}30 \\
40 \\
40 \\
40 \\
30\end{array}$ & $\begin{array}{l}-- \\
-- \\
-- \\
--\end{array}$ \\
\hline $\begin{array}{l}.00 \\
.01\end{array}$ & $\begin{array}{r}.64 \\
.35\end{array}$ & $\begin{array}{l}.01 \\
.02\end{array}$ & $\begin{array}{l}180 \\
110\end{array}$ & $\begin{array}{l}10 \\
30\end{array}$ & -- \\
\hline
\end{tabular}


Table 5.--Water-level records and chemical [We11 number 24 on figure 2. Local well number is SB00506425ACB2.

\begin{tabular}{|c|c|c|c|c|c|c|c|c|}
\hline $\begin{array}{l}\text { DATE OF } \\
\text { WATER-LEVEL } \\
\text { MEASUREMENT }\end{array}$ & $\begin{array}{l}\text { WATER } \\
\text { LEVEL } \\
\text { (FEET } \\
\text { BELOW } \\
\text { LAND } \\
\text { SURFACE) }\end{array}$ & $\begin{array}{c}\text { DATE } \\
\text { OF } \\
\text { SAMPLE }\end{array}$ & $\begin{array}{l}\text { SPE- } \\
\text { CIFIC } \\
\text { CON- } \\
\text { DUCT- } \\
\text { ANCE } \\
\text { (MICRO- } \\
\text { MHOS) }\end{array}$ & $\begin{array}{c}\text { PH } \\
\text { (UNITS) }\end{array}$ & $\begin{array}{l}\text { TEMPER- } \\
\text { ATURE } \\
\text { (DEG C) }\end{array}$ & $\begin{array}{l}\text { HARD- } \\
\text { NESS } \\
\text { (MG/L } \\
\text { AS } \\
\text { CACC3) }\end{array}$ & $\begin{array}{l}\text { HARD- } \\
\text { NESS, } \\
\text { NONCAR- } \\
\text { BONATE } \\
\text { (MG/L } \\
\text { CACO3) }\end{array}$ & $\begin{array}{l}\text { CALCIUM, } \\
\text { DIS- } \\
\text { SOLVED } \\
\text { (MG/L } \\
\text { AS CA) }\end{array}$ \\
\hline $\begin{array}{l}74-04-03 \\
74-05-08 \\
74-06-13 \\
74-07-23 \\
74-08-27\end{array}$ & $\begin{array}{l}33.42 \\
33.49 \\
33.82 \\
33.95 \\
33.70\end{array}$ & $\begin{array}{l}74-04-03 \\
74-05-08 \\
74-06-13 \\
74-07-23 \\
74-08-27\end{array}$ & $\begin{array}{l}2530 \\
2770 \\
2560 \\
2560 \\
2500\end{array}$ & $\begin{array}{l}7.2 \\
7.3 \\
7.4 \\
7.3 \\
7.1\end{array}$ & $\begin{array}{l}11.5 \\
12.5 \\
13.0 \\
13.5 \\
12.5\end{array}$ & $\begin{array}{r}1100 \\
1000 \\
940 \\
900 \\
850\end{array}$ & $\begin{array}{l}700 \\
650 \\
590 \\
540 \\
500\end{array}$ & $\begin{array}{l}260 \\
250 \\
230 \\
220 \\
200\end{array}$ \\
\hline $\begin{array}{l}74-10-03 \\
74-11-11 \\
74-12-05 \\
75-02-06 \\
75-05-14\end{array}$ & $\begin{array}{l}33.08 \\
33.08 \\
33.16 \\
33.52 \\
34.18\end{array}$ & $\begin{array}{l}74-10-03 \\
74-11-11 \\
74-12-05 \\
75-02-06 \\
75-05-14\end{array}$ & $\begin{array}{l}2460 \\
2500 \\
2700 \\
2700 \\
3100\end{array}$ & $\begin{array}{l}7 .- \\
7.2 \\
7.1 \\
7.1 \\
7.2\end{array}$ & $\begin{array}{l}12.5 \\
12.0 \\
12.0 \\
11.5 \\
12.5\end{array}$ & $\begin{array}{l}860 \\
840 \\
860 \\
870 \\
870\end{array}$ & $\begin{array}{l}510 \\
490 \\
510 \\
520 \\
530\end{array}$ & $\begin{array}{l}210 \\
210 \\
210 \\
210 \\
230\end{array}$ \\
\hline $\begin{array}{l}75-08-11 \\
75-12-01 \\
76-03-29 \\
76-06-29 \\
76-09-22\end{array}$ & $\begin{array}{l}34.43 \\
33.30 \\
33.70 \\
34.42 \\
34.08\end{array}$ & $\begin{array}{l}75-08-11 \\
75-12-01 \\
76-03-29 \\
76-06-29 \\
76-09-22\end{array}$ & $\begin{array}{l}3000 \\
3000 \\
2675 \\
2900 \\
2800\end{array}$ & $\begin{array}{l}7.3 \\
7.4 \\
7.4 \\
6.6 \\
7.1\end{array}$ & $\begin{array}{l}12.5 \\
11.5 \\
12.0 \\
13.0 \\
13.5\end{array}$ & $\begin{array}{r}900 \\
910 \\
1000 \\
1000 \\
1100\end{array}$ & $\begin{array}{l}550 \\
560 \\
660 \\
680 \\
730\end{array}$ & $\begin{array}{l}210 \\
220 \\
250 \\
250 \\
270\end{array}$ \\
\hline $\begin{array}{l}76-12-08 \\
77-03-08 \\
77-06-15 \\
77-09-13 \\
77-12-06\end{array}$ & $\begin{array}{l}33.39 \\
33.90 \\
34.67 \\
34.52 \\
33.84\end{array}$ & $\begin{array}{l}76-12-08 \\
77-03-08 \\
77-06-15 \\
77-09-13 \\
77-12-06\end{array}$ & $\begin{array}{l}2900 \\
3000 \\
3000 \\
3000 \\
3000\end{array}$ & $\begin{array}{l}7.3 \\
7.4 \\
7.4 \\
7.5 \\
7.2\end{array}$ & $\begin{array}{l}12.0 \\
12.5 \\
13.0 \\
13.0 \\
12.0\end{array}$ & $\begin{array}{r}1200 \\
1000 \\
980 \\
1100 \\
1100\end{array}$ & $\begin{array}{l}780 \\
660 \\
610 \\
680 \\
660\end{array}$ & $\begin{array}{l}280 \\
250 \\
240 \\
260 \\
240\end{array}$ \\
\hline $\begin{array}{l}78-03-09 \\
78-06-20\end{array}$ & $\begin{array}{l}33.84 \\
34.11\end{array}$ & $\begin{array}{l}78-03-09 \\
78-05-20\end{array}$ & $\begin{array}{l}3000 \\
3000\end{array}$ & $\begin{array}{l}7.2 \\
7.3\end{array}$ & $\begin{array}{l}12.5 \\
13.0\end{array}$ & $\begin{array}{l}1100 \\
1100\end{array}$ & $\begin{array}{l}710 \\
680\end{array}$ & $\begin{array}{l}260 \\
250\end{array}$ \\
\hline $\begin{array}{l}\text { DATE OF } \\
\text { WATER-LEVEL } \\
\text { MEASUREMENT }\end{array}$ & $\begin{array}{l}\text { WATER } \\
\text { LEVEL } \\
\text { (FEET } \\
\text { BELOW } \\
\text { LAND } \\
\text { SURFACE) }\end{array}$ & $\begin{array}{c}\text { DATE } \\
\text { OF } \\
\text { SAMPLE }\end{array}$ & $\begin{array}{l}\text { FLUO- } \\
\text { RIDE, } \\
\text { DIS- } \\
\text { SOLVED } \\
(M G / L \\
\text { AS F) }\end{array}$ & $\begin{array}{l}\text { SILICA, } \\
\text { DIS- } \\
\text { SOLVED } \\
\text { (MG'L } \\
\text { AS } \\
\text { SIO2) }\end{array}$ & $\begin{array}{c}\text { SOL IDS, } \\
\text { SUM OF } \\
\text { CONSTI- } \\
\text { TUENTS, } \\
\text { DIS- } \\
\text { SOLVED } \\
\text { (MG/L) }\end{array}$ & $\begin{array}{c}\text { NITRO- } \\
\text { GEN, } \\
\text { NITRATE } \\
\text { DIS- } \\
\text { SOLVED } \\
\text { (MG/L } \\
\text { AS N) }\end{array}$ & $\begin{array}{c}\text { NITRO- } \\
\text { GEN, } \\
\text { NITRITE } \\
\text { DIS- } \\
\text { SOLVED } \\
\text { (MG/L } \\
\text { AS N) }\end{array}$ & $\begin{array}{c}\text { NITRO- } \\
\text { GEN, } \\
\text { NO2+NO3 } \\
\text { OIS- } \\
\text { SOLVED } \\
\text { (MG/L } \\
\text { AS N) }\end{array}$ \\
\hline $\begin{array}{l}74-04-03 \\
74-05-08 \\
74-06-13 \\
74-07-23 \\
74-08-27\end{array}$ & $\begin{array}{l}33.42 \\
33.49 \\
33.82 \\
33.95 \\
33.70\end{array}$ & $\begin{array}{l}74-04-03 \\
74-05-08 \\
74-06-13 \\
74-07-23 \\
74-08-27\end{array}$ & $\begin{array}{l}1.5 \\
1.5 \\
1.4 \\
1.6 \\
1.3\end{array}$ & $\begin{array}{l}22 \\
23 \\
23 \\
24 \\
19\end{array}$ & $\begin{array}{l}2250 \\
2100 \\
1960 \\
1890 \\
1780\end{array}$ & $\begin{array}{l}7.3 \\
7.7 \\
7.1 \\
4.2\end{array}$ & $\begin{array}{l}.04 \\
.02 \\
.02 \\
.01\end{array}$ & $\begin{array}{l}7.3 \\
7.7 \\
7.1 \\
7.1 \\
4.2\end{array}$ \\
\hline $\begin{array}{l}74-10-03 \\
74-11-11 \\
74-12-05 \\
75-02-06 \\
75-05-14\end{array}$ & $\begin{array}{l}33.08 \\
33.08 \\
33.16 \\
33.52 \\
34.18\end{array}$ & $\begin{array}{l}74-10-03 \\
74-11-11 \\
74-12-05 \\
75-02-06 \\
75-05-14\end{array}$ & $\begin{array}{l}1.5 \\
1.5 \\
1.6 \\
1.5 \\
1.5\end{array}$ & $\begin{array}{l}24 \\
23 \\
23 \\
23 \\
23\end{array}$ & $\begin{array}{l}1830 \\
1820 \\
1820 \\
1890 \\
1870\end{array}$ & $\begin{array}{l}6.5 \\
6.7 \\
7.3 \\
9.9\end{array}$ & $\begin{array}{l}.01 \\
.02 \\
.00 \\
.00 \\
.00\end{array}$ & $\begin{array}{l}6.7 \\
6.5 \\
6.7 \\
7.3 \\
9.9\end{array}$ \\
\hline $\begin{array}{l}75-08-11 \\
75-12-01 \\
76-03-29 \\
76-06-29 \\
76-09-22\end{array}$ & $\begin{array}{l}34.43 \\
33.30 \\
33.70 \\
34.42 \\
34.08\end{array}$ & $\begin{array}{l}75-08-11 \\
75-12-01 \\
76-03-29 \\
76-06-29 \\
76-09-22\end{array}$ & $\begin{array}{l}1.4 \\
1.6 \\
1.6 \\
1.6 \\
1.6\end{array}$ & $\begin{array}{l}22 \\
22 \\
22 \\
21 \\
22\end{array}$ & $\begin{array}{l}1830 \\
1950 \\
2130 \\
2250 \\
2390\end{array}$ & $\begin{array}{l}6.8 \\
7.0 \\
8.5 \\
8.8 \\
9.4\end{array}$ & $\begin{array}{l}.01 \\
.01 \\
.00 \\
.00 \\
.01\end{array}$ & $\begin{array}{l}5.8 \\
7.0 \\
8.5 \\
8.8 \\
9.4\end{array}$ \\
\hline $\begin{array}{l}76-12-08 \\
77-03-08 \\
77-06-15 \\
77-09-13 \\
77-12-06\end{array}$ & $\begin{array}{l}33.39 \\
33.90 \\
34.67 \\
34.52 \\
33.84\end{array}$ & $\begin{array}{l}76-12-08 \\
77-03-08 \\
77-06-15 \\
77-09-13 \\
77-12-06\end{array}$ & $\begin{array}{l}1.6 \\
1.7 \\
1.7 \\
1.8 \\
1.8\end{array}$ & $\begin{array}{l}21 \\
23 \\
21 \\
23 \\
22\end{array}$ & $\begin{array}{l}2540 \\
2320 \\
2290 \\
2340 \\
2350\end{array}$ & $\begin{array}{l}12 \\
10 \\
8.6 \\
10 \\
11\end{array}$ & $\begin{array}{l}.00 \\
.00 \\
.00 \\
.00 \\
.01\end{array}$ & $\begin{array}{l}12 \\
10 \\
8.6 \\
10 \\
11\end{array}$ \\
\hline $\begin{array}{l}78-03-09 \\
78-06-20\end{array}$ & $\begin{array}{l}33.84 \\
34.11\end{array}$ & $\begin{array}{l}78-03-09 \\
78-06-20\end{array}$ & $\begin{array}{l}1.8 \\
1.6\end{array}$ & $\begin{array}{l}21 \\
19\end{array}$ & $\begin{array}{l}2350 \\
2370\end{array}$ & $\begin{array}{l}9.7 \\
9.5\end{array}$ & $\begin{array}{l}.00 \\
.02\end{array}$ & $\begin{array}{l}9.7 \\
9.5\end{array}$ \\
\hline
\end{tabular}


analyses of water from observation wells--Continued

Depth is $64 \mathrm{ft}$. Interval open to the aquifer is 60 to $64 \mathrm{ft}$ ]

\begin{tabular}{|c|c|c|c|c|c|c|c|c|}
\hline $\begin{array}{l}\text { MAGNE- } \\
\text { SIUN, } \\
\text { CIS- } \\
\text { SU:VED } \\
\text { MGGIL } \\
\text { S MG! }\end{array}$ & $\begin{array}{l}\text { SOOIUM, } \\
\text { OIS- } \\
\text { SOLVED } \\
\text { (MG/L } \\
\text { AS NA) }\end{array}$ & $\begin{array}{l}\text { SODIUM } \\
\text { AD- } \\
\text { SORP- } \\
\text { TION } \\
\text { RATIO }\end{array}$ & $\begin{array}{l}\text { POTAS- } \\
\text { SIUM, } \\
\text { DIS- } \\
\text { SOLVED } \\
\text { (MG/L } \\
\text { AS K) }\end{array}$ & $\begin{array}{l}\text { BI UAR- } \\
\text { BONATE } \\
\text { (MG/L } \\
\text { AS } \\
\text { H乞Oß) }\end{array}$ & $\begin{array}{c}\text { CAR- } \\
\text { BONATE } \\
\text { (MG/L } \\
\text { AS CO3) }\end{array}$ & $\begin{array}{l}\text { ALKA- } \\
\text { LINITY } \\
\text { (MG/L } \\
\text { AS } \\
\text { CACO3) }\end{array}$ & $\begin{array}{c}\text { SULFATE, } \\
\text { DIS- } \\
\text { SOLVED } \\
\text { (MG/L } \\
\text { AS SO4) }\end{array}$ & $\begin{array}{l}\text { CHLO- } \\
\text { RIDE, } \\
\text { DIS- } \\
\text { SOLVED } \\
\text { (MG/L } \\
\text { AS CL) }\end{array}$ \\
\hline $\begin{array}{r}100 \\
04 \\
80 \\
84 \\
85\end{array}$ & $\begin{array}{l}320 \\
280 \\
280 \\
280 \\
260\end{array}$ & $\begin{array}{l}4.3 \\
3.8 \\
4.0 \\
4.1 \\
3.0\end{array}$ & $\begin{array}{l}5.9 \\
6.0 \\
5.5 \\
4.2 \\
5.9\end{array}$ & $\begin{array}{l}439 \\
436 \\
429 \\
428 \\
429\end{array}$ & $\begin{array}{r}0 \\
0 \\
0 \\
-- \\
--\end{array}$ & $\begin{array}{l}360 \\
358 \\
352 \\
351 \\
352\end{array}$ & $\begin{array}{r}1200 \\
1100 \\
1000 \\
940 \\
890\end{array}$ & $\begin{array}{r}96 \\
100 \\
92 \\
95 \\
91\end{array}$ \\
\hline $\begin{array}{l}81 \\
7 \hat{\mathrm{i}} \\
8 \hat{2} \\
85 \\
72\end{array}$ & $\begin{array}{l}260 \\
270 \\
270 \\
290 \\
270\end{array}$ & $\begin{array}{l}3.9 \\
4.1 \\
4.0 \\
4.3 \\
4.0\end{array}$ & $\begin{array}{l}6.3 \\
4.4 \\
5.4 \\
6.1 \\
5.2\end{array}$ & $\begin{array}{l}426 \\
426 \\
430 \\
432 \\
421\end{array}$ & $\begin{array}{l}-- \\
-- \\
-- \\
0\end{array}$ & $\begin{array}{l}349 \\
349 \\
353 \\
354 \\
345\end{array}$ & $\begin{array}{l}920 \\
910 \\
900 \\
930 \\
920\end{array}$ & $\begin{array}{l}89 \\
83 \\
90 \\
98 \\
94\end{array}$ \\
\hline $\begin{array}{r}92 \\
87 \\
96 \\
100 \\
100\end{array}$ & $\begin{array}{l}270 \\
290 \\
300 \\
320 \\
330\end{array}$ & $\begin{array}{l}3.9 \\
4.2 \\
4.1 \\
4.3 \\
4.4\end{array}$ & $\begin{array}{l}6.2 \\
6.5 \\
7.1 \\
7.0 \\
7.3\end{array}$ & $\begin{array}{l}426 \\
425 \\
435 \\
434 \\
435\end{array}$ & $\begin{array}{l}0 \\
0 \\
0 \\
0 \\
0\end{array}$ & $\begin{array}{l}349 \\
349 \\
357 \\
356 \\
357\end{array}$ & $\begin{array}{r}900 \\
1000 \\
1100 \\
1200 \\
1300\end{array}$ & $\begin{array}{l}90 \\
85 \\
97 \\
97 \\
98\end{array}$ \\
\hline $\begin{array}{r}110 \\
98 \\
93 \\
100 \\
110\end{array}$ & $\begin{array}{l}340 \\
360 \\
250 \\
360 \\
360\end{array}$ & $\begin{array}{l}4.4 \\
4.9 \\
4.9 \\
4.8 \\
4.8\end{array}$ & $\begin{array}{l}7.5 \\
6.6 \\
6.7 \\
6.5 \\
6.9\end{array}$ & $\begin{array}{l}453 \\
454 \\
460 \\
470 \\
480\end{array}$ & $\begin{array}{l}0 \\
0 \\
0 \\
0 \\
0\end{array}$ & $\begin{array}{l}372 \\
372 \\
380 \\
390 \\
390\end{array}$ & $\begin{array}{l}1400 \\
1200 \\
1200 \\
1200 \\
1200\end{array}$ & $\begin{array}{l}100 \\
110 \\
110 \\
110 \\
120\end{array}$ \\
\hline $\begin{array}{l}110 \\
110\end{array}$ & $\begin{array}{l}350 \\
380\end{array}$ & $\begin{array}{l}4.6 \\
5.0\end{array}$ & $\begin{array}{l}7.4 \\
8.2\end{array}$ & $\begin{array}{l}480 \\
480\end{array}$ & $\begin{array}{l}0 \\
0\end{array}$ & $\begin{array}{l}390 \\
390\end{array}$ & $\begin{array}{l}1200 \\
1200\end{array}$ & $\begin{array}{l}120 \\
120\end{array}$ \\
\hline $\begin{array}{l}\text { NITRO- } \\
\text { GEN, } \\
\text { AMMONIA } \\
\text { DIS- } \\
\text { SOLVED } \\
\text { (MG/L } \\
\text { AS N! }\end{array}$ & $\begin{array}{l}\text { NITRO- } \\
\text { GEN,AM- } \\
\text { MONIA + } \\
\text { ORGANIC } \\
\text { DIS. } \\
(M G / L \\
\text { AS N) }\end{array}$ & $\begin{array}{l}\text { PHOS- } \\
\text { PHORUS, } \\
\text { ORTHO, } \\
\text { DIS- } \\
\text { SOLVED } \\
\text { (MG/L } \\
\text { AS P) }\end{array}$ & $\begin{array}{l}\text { IRON, } \\
\text { DIS- } \\
\text { SOLVED } \\
\text { (UG/L } \\
\text { AS FE) }\end{array}$ & $\begin{array}{l}\text { MANGG- } \\
\text { NESE, } \\
\text { DIS- } \\
\text { SOLVED } \\
\text { (UG/L } \\
\text { AS MN) }\end{array}$ & $\begin{array}{l}\text { CARBON, } \\
\text { ORGANIC } \\
\text { DIS- } \\
\text { SOLVED } \\
\text { (MG/L } \\
\text { AS C) }\end{array}$ & & & \\
\hline $\begin{array}{l}.08 \\
.12 \\
.05 \\
. . \\
.04\end{array}$ & $\begin{array}{l}.71 \\
.62 \\
.72 \\
.0 \\
.66\end{array}$ & $\begin{array}{l}.03 \\
.02 \\
.05 \\
.06 \\
.03\end{array}$ & $\begin{array}{r}160 \\
70 \\
20 \\
50 \\
20\end{array}$ & $\begin{array}{r}150 \\
30 \\
0 \\
0 \\
0\end{array}$ & $\begin{array}{l}-- \\
-- \\
-- \\
--\end{array}$ & & & \\
\hline $\begin{array}{l}.02 \\
.01 \\
.01 \\
.01 \\
.02\end{array}$ & $\begin{array}{l}.35 \\
.60 \\
.33 \\
.48 \\
.10\end{array}$ & $\begin{array}{l}.03 \\
.04 \\
.04 \\
.03 \\
.04\end{array}$ & $\begin{array}{l}60 \\
20 \\
40 \\
10 \\
40\end{array}$ & $\begin{array}{r}0 \\
0 \\
10 \\
20 \\
5\end{array}$ & $\begin{array}{l}-- \\
-- \\
-- \\
--\end{array}$ & & & \\
\hline $\begin{array}{l}.00 \\
.09 \\
.02 \\
.00 \\
.00\end{array}$ & $\begin{array}{l}.30 \\
.97 \\
.49 \\
.23 \\
.40\end{array}$ & $\begin{array}{l}.04 \\
.05 \\
.03 \\
.07 \\
.04\end{array}$ & $\begin{array}{l}10 \\
30 \\
10 \\
10 \\
30\end{array}$ & $\begin{array}{r}0 \\
10 \\
0 \\
0 \\
10\end{array}$ & $\begin{array}{r}-- \\
-- \\
-- \\
3.9 \\
5.3\end{array}$ & & & \\
\hline $\begin{array}{l}.00 \\
.01 \\
.04 \\
.01 \\
.01\end{array}$ & $\begin{array}{l}1.1 \\
.75 \\
.30 \\
.54 \\
.22\end{array}$ & $\begin{array}{l}.02 \\
.04 \\
.05 \\
.02 \\
.02\end{array}$ & $\begin{array}{r}80 \\
50 \\
90 \\
180 \\
200\end{array}$ & $\begin{array}{l}10 \\
10 \\
10 \\
10 \\
20\end{array}$ & $\begin{array}{l}-- \\
-- \\
-- \\
--\end{array}$ & & & \\
\hline $\begin{array}{l}.00 \\
.01\end{array}$ & $1.7^{62}$ & $\begin{array}{l}.02 \\
.07\end{array}$ & $\begin{array}{l}100 \\
230\end{array}$ & $\begin{array}{r}20 \\
5\end{array}$ & $\begin{array}{l}-- \\
--\end{array}$ & & & \\
\hline
\end{tabular}


Table 5.--Water-level records and chemical [Well number 25 on figure 2. Local well number is SB00506425ACB3.

\begin{tabular}{|c|c|c|c|c|c|c|c|c|}
\hline $\begin{array}{l}\text { DATE OF } \\
\text { WATER-LEVEL } \\
\text { MEASUREMENT }\end{array}$ & $\begin{array}{l}\text { WATER } \\
\text { LEVEL } \\
\text { (FEET } \\
\text { BELOW } \\
\text { LAND } \\
\text { SURFACE) }\end{array}$ & $\begin{array}{c}\text { DATE } \\
\text { OF } \\
\text { SAMPLE }\end{array}$ & $\begin{array}{l}\text { SPE- } \\
\text { CIFIC } \\
\text { CON- } \\
\text { DUCT- } \\
\text { ANCE } \\
\text { (MICRO- } \\
\text { MHOS) }\end{array}$ & $\begin{array}{c}\text { PH } \\
\text { (UNITS) }\end{array}$ & $\begin{array}{l}\text { TEMPER- } \\
\text { ATURE } \\
\text { (DEG C) }\end{array}$ & $\begin{array}{l}\text { HARD- } \\
\text { NESS } \\
\text { (MG/L } \\
\text { AS } \\
\text { CAC03) }\end{array}$ & $\begin{array}{l}\text { HARD- } \\
\text { NESS, } \\
\text { NONCAR- } \\
\text { BONATE } \\
\text { (MG/L } \\
\text { CACO3) }\end{array}$ & $\begin{array}{l}\text { CALCIUM, } \\
\text { DIS- } \\
\text { SOLVED } \\
\text { (MG/L } \\
\text { AS CA) }\end{array}$ \\
\hline $\begin{array}{l}74-04-03 \\
74-05-08 \\
74-06-13 \\
74-07-23 \\
74-08-27\end{array}$ & $\begin{array}{l}33.61 \\
33.65 \\
34.05 \\
34.17 \\
33.91\end{array}$ & $\begin{array}{l}74-04-03 \\
74-05-08 \\
74-06-13 \\
74-07-23 \\
74-08-27\end{array}$ & $\begin{array}{l}3090 \\
3000 \\
3150 \\
3140 \\
3190\end{array}$ & $\begin{array}{r}7.1 \\
7.3 \\
7.4 \\
7.3 \\
--\end{array}$ & $\begin{array}{l}11.5 \\
13.0 \\
12.5 \\
13.0 \\
12.0\end{array}$ & $\begin{array}{l}1300 \\
1200 \\
1300 \\
1300 \\
1500\end{array}$ & $\begin{array}{r}910 \\
870 \\
1000 \\
1000 \\
1100\end{array}$ & $\begin{array}{l}320 \\
300 \\
330 \\
330 \\
360\end{array}$ \\
\hline $\begin{array}{l}74-10-03 \\
74-11-11 \\
74-12-05 \\
75-02-06 \\
75-05-14\end{array}$ & $\begin{array}{l}33.26 \\
33.24 \\
33.33 \\
33.69 \\
34.36\end{array}$ & $\begin{array}{l}74-10-03 \\
74-11-11 \\
74-12-05 \\
75-02-06 \\
75-05-14\end{array}$ & $\begin{array}{l}3210 \\
3400 \\
3200 \\
3600 \\
4200\end{array}$ & $\begin{array}{l}7 . \overline{2} \\
7.0 \\
7.0 \\
6.8\end{array}$ & $\begin{array}{l}12.0 \\
11.5 \\
12.0 \\
11.5 \\
12.5\end{array}$ & $\begin{array}{l}1300 \\
1500 \\
1600 \\
1500 \\
1400\end{array}$ & $\begin{array}{l}1000 \\
1100 \\
1200 \\
1100 \\
1000\end{array}$ & $\begin{array}{l}370 \\
390 \\
400 \\
380 \\
360\end{array}$ \\
\hline $\begin{array}{l}75-08-11 \\
76-06-29 \\
76-09-22 \\
76-12-08 \\
77-03-08\end{array}$ & $\begin{array}{l}32.55 \\
34.56 \\
34.26 \\
33.56 \\
34.05\end{array}$ & $\begin{array}{l}75-08-11 \\
76-06-29 \\
76-09-22 \\
76-12-08 \\
77-03-08\end{array}$ & $\begin{array}{l}4000 \\
2900 \\
2700 \\
2800 \\
2900\end{array}$ & $\begin{array}{l}7.0 \\
6.1 \\
6.9 \\
6.7 \\
7.2\end{array}$ & $\begin{array}{l}13.5 \\
12.5 \\
13.5 \\
12.0 \\
12.5\end{array}$ & $\begin{array}{l}1400 \\
1100 \\
1100 \\
1100 \\
1100\end{array}$ & $\begin{array}{l}990 \\
730 \\
770 \\
730 \\
740\end{array}$ & $\begin{array}{l}360 \\
300 \\
310 \\
310 \\
290\end{array}$ \\
\hline $\begin{array}{l}77-06-15 \\
77-09-13 \\
77-12-06 \\
78-03-09 \\
78-06-20\end{array}$ & $\begin{array}{l}34.80 \\
34.70 \\
33.74 \\
34.01 \\
34.25\end{array}$ & $\begin{array}{l}77-06-15 \\
77-09-13 \\
77-12-06 \\
78-03-09 \\
78-06-20\end{array}$ & $\begin{array}{l}3000 \\
2900 \\
3000 \\
3000 \\
3000\end{array}$ & $\begin{array}{l}6.9 \\
7.2 \\
6.9 \\
7.0 \\
7.1\end{array}$ & $\begin{array}{l}12.5 \\
13.0 \\
12.0 \\
12.5 \\
13.0\end{array}$ & $\begin{array}{l}1100 \\
1200 \\
1100 \\
1100 \\
1200\end{array}$ & $\begin{array}{r}750 \\
820 \\
760 \\
1000 \\
810\end{array}$ & $\begin{array}{l}280 \\
300 \\
280 \\
270 \\
300\end{array}$ \\
\hline $\begin{array}{l}\text { DATE OF } \\
\text { WATER-LEVEL } \\
\text { MEASUREMENT }\end{array}$ & $\begin{array}{l}\text { WATER } \\
\text { LEVEL } \\
\text { (FEET } \\
\text { BELOW } \\
\text { LAND } \\
\text { SURFACE) }\end{array}$ & $\begin{array}{c}\text { DATE } \\
\text { OF } \\
\text { SAMPLE }\end{array}$ & $\begin{array}{l}\text { FLUO- } \\
\text { RIDE, } \\
\text { DIS- } \\
\text { SOLVED } \\
\text { (MG/L } \\
\text { AS F) }\end{array}$ & $\begin{array}{l}\text { SILICA, } \\
\text { DIS- } \\
\text { SOLVED } \\
\text { (MG/L } \\
\text { AS } \\
\text { SI02) }\end{array}$ & $\begin{array}{c}\text { SOLIDS, } \\
\text { SUM OFF } \\
\text { CONSTI- } \\
\text { TUENTS, } \\
\text { DIS- } \\
\text { SOLVED } \\
\text { (MG/L) }\end{array}$ & $\begin{array}{c}\text { NITRO- } \\
\text { GEN, } \\
\text { NITRATE } \\
\text { DIS- } \\
\text { SOLVED } \\
\text { (MG/L } \\
\text { AS N) }\end{array}$ & $\begin{array}{c}\text { NITRO- } \\
\text { GEN, } \\
\text { NITRITE } \\
\text { DIS- } \\
\text { SOLVED } \\
\text { (MG/L } \\
\text { AS N) }\end{array}$ & $\begin{array}{c}\text { NITRO- } \\
\text { GEN, } \\
\text { NO2+N03 } \\
\text { DIS- } \\
\text { SOLVED } \\
\text { (MG/L } \\
\text { AS N) }\end{array}$ \\
\hline $\begin{array}{l}74-04-03 \\
74-05-08 \\
74-06-13 \\
74-07-23 \\
74-08-27\end{array}$ & $\begin{array}{l}33.61 \\
33.65 \\
34.05 \\
34.17 \\
33.91\end{array}$ & $\begin{array}{l}74-04-03 \\
74-05-08 \\
74-06-13 \\
74-07-23 \\
74-08-27\end{array}$ & $\begin{array}{l}1.6 \\
.9 \\
2.6 \\
1.6 \\
1.5\end{array}$ & $\begin{array}{l}22 \\
23 \\
23 \\
23 \\
23\end{array}$ & $\begin{array}{l}2410 \\
2290 \\
2550 \\
2430 \\
2570\end{array}$ & $\begin{array}{l}28 \\
13 \\
35\end{array}$ & $\begin{array}{l}.22 \\
.08 \\
.05 \\
. \overline{.02}\end{array}$ & $\begin{array}{l}28 \\
13 \\
35 \\
35 \\
25\end{array}$ \\
\hline $\begin{array}{l}74-10-03 \\
74-11-11 \\
74-12-05 \\
75-02-06 \\
75-05-14\end{array}$ & $\begin{array}{l}33.26 \\
33.24 \\
33.33 \\
33.69 \\
34.36\end{array}$ & $\begin{array}{l}74-10-03 \\
74-11-11 \\
74-12-05 \\
75-02-06 \\
75-05-14\end{array}$ & $\begin{array}{l}1.6 \\
1.5 \\
.9 \\
1.1 \\
1.1\end{array}$ & $\begin{array}{l}23 \\
22 \\
18 \\
26 \\
25\end{array}$ & $\begin{array}{l}2560 \\
2680 \\
2690 \\
2660 \\
2530\end{array}$ & $3_{38^{5}} .-{ }^{--}$ & $\begin{array}{l}.01 \\
.01 \\
.00 \\
.00 \\
.00\end{array}$ & $\begin{array}{l}37 \\
50 \\
38 \\
35\end{array} .42$ \\
\hline $\begin{array}{l}75-08-11 \\
76-06-29 \\
76-09-22 \\
76-12-08 \\
77-03-08\end{array}$ & $\begin{array}{l}32.55 \\
34.56 \\
34.26 \\
33.56 \\
34.05\end{array}$ & $\begin{array}{l}75-08-11 \\
76-06-29 \\
76-09-22 \\
76-12-08 \\
77-03-08\end{array}$ & $\begin{array}{r}.9 \\
.9 \\
1.0 \\
.7 \\
.8\end{array}$ & $\begin{array}{l}27 \\
29 \\
27 \\
31 \\
31\end{array}$ & $\begin{array}{l}2470 \\
2260 \\
2250 \\
2250 \\
2120\end{array}$ & $\begin{array}{l}31 \\
22 \\
22 \\
30 \\
23\end{array}$ & $\begin{array}{l}.01 \\
.00 \\
.01 \\
.01 \\
.00\end{array}$ & $\begin{array}{l}31 \\
22 \\
22 \\
30 \\
23\end{array}$ \\
\hline $\begin{array}{l}77-06-15 \\
77-09-13 \\
77-12-06 \\
78-03-09 \\
78-06-20\end{array}$ & $\begin{array}{l}34.80 \\
34.70 \\
33.74 \\
34.01 \\
34.25\end{array}$ & $\begin{array}{l}77-06-15 \\
77-09-13 \\
77-12-06 \\
78-03-09 \\
78-06-20\end{array}$ & $\begin{array}{l}1.0 \\
.9 \\
1.2 \\
1.3 \\
1.2\end{array}$ & $\begin{array}{l}27 \\
30 \\
26 \\
24 \\
22\end{array}$ & $\begin{array}{l}2180 \\
2150 \\
2310 \\
2080 \\
2330\end{array}$ & $\begin{array}{l}28 \\
16 \\
26 \\
22 \\
22\end{array}$ & $\begin{array}{l}.01 \\
.00 \\
.01 \\
.00 \\
.02\end{array}$ & $\begin{array}{l}28 \\
16 \\
26 \\
22 \\
22\end{array}$ \\
\hline
\end{tabular}


analyses of water from observation wells--Continued Depth is $46 \mathrm{ft}$. Interval open to the aquifer is 42 to $46 \mathrm{ft}$ ]

\begin{tabular}{|c|c|c|c|c|c|c|c|c|}
\hline $\begin{array}{l}\text { MAGNE- } \\
\text { SIUM, } \\
\text { DIS- } \\
\text { SOLVED } \\
\text { (MG/L } \\
\text { AS MG) }\end{array}$ & $\begin{array}{l}\text { SODIUM, } \\
\text { DIS- } \\
\text { SOLVED } \\
\text { (MG/L } \\
\text { AS NA) }\end{array}$ & $\begin{array}{l}\text { SODIUM } \\
\text { AD- } \\
\text { SORP- } \\
\text { TION } \\
\text { RATIO }\end{array}$ & $\begin{array}{l}\text { POTAS- } \\
\text { SIUM, } \\
\text { DIS- } \\
\text { SOLVED } \\
\text { (MG/L } \\
\text { AS K) }\end{array}$ & $\begin{array}{l}\text { BICAR- } \\
\text { BONATE } \\
\text { (MG/L } \\
\text { AS } \\
\text { HCO3) }\end{array}$ & $\begin{array}{c}\text { CAR- } \\
\text { BONATE } \\
\text { (MG/L } \\
\text { AS CO3) }\end{array}$ & $\begin{array}{l}\text { ALKA- } \\
\text { LINITY } \\
\text { (MG/L } \\
\text { AS } \\
\text { CAC03) }\end{array}$ & $\begin{array}{l}\text { SULFATE, } \\
\text { DIS- } \\
\text { SOLVED } \\
\text { (MG/L } \\
\text { AS SO4) }\end{array}$ & $\begin{array}{l}\text { CHLO- } \\
\text { RIDE, } \\
\text { DIS- } \\
\text { SOLVED } \\
\text { (MG/L } \\
\text { AS CL) }\end{array}$ \\
\hline $\begin{array}{l}110 \\
110 \\
120 \\
120 \\
140\end{array}$ & $\begin{array}{l}290 \\
290 \\
290 \\
270 \\
270\end{array}$ & $\begin{array}{l}3.6 \\
3.6 \\
3.5 \\
3.2 \\
3.1\end{array}$ & $\begin{array}{l}5.9 \\
5.5 \\
5.6 \\
3.6 \\
7.1\end{array}$ & $\begin{array}{l}421 \\
401 \\
381 \\
385 \\
417\end{array}$ & $\begin{array}{r}0 \\
0 \\
0 \\
-- \\
--\end{array}$ & $\begin{array}{l}345 \\
329 \\
313 \\
316 \\
342\end{array}$ & $\begin{array}{l}1200 \\
1200 \\
1300 \\
1200 \\
1300\end{array}$ & $\begin{array}{l}130 \\
110 \\
140 \\
140 \\
150\end{array}$ \\
\hline $\begin{array}{l}100 \\
130 \\
150 \\
140 \\
130\end{array}$ & $\begin{array}{l}270 \\
250 \\
240 \\
260 \\
260\end{array}$ & $\begin{array}{l}3.2 \\
2.8 \\
2.6 \\
2.9 \\
3.0\end{array}$ & $\begin{array}{l}6.0 \\
3.6 \\
5.1 \\
6.0 \\
6.3\end{array}$ & $\begin{array}{l}404 \\
459 \\
478 \\
465 \\
483\end{array}$ & $\begin{array}{l}-- \\
-- \\
-- \\
0\end{array}$ & $\begin{array}{l}331 \\
376 \\
392 \\
381 \\
396\end{array}$ & $\begin{array}{l}1300 \\
1300 \\
1300 \\
1300 \\
1200\end{array}$ & $\begin{array}{l}130 \\
140 \\
150 \\
150 \\
150\end{array}$ \\
\hline $\begin{array}{r}120 \\
89 \\
89 \\
91 \\
84\end{array}$ & $\begin{array}{l}250 \\
270 \\
280 \\
280 \\
280\end{array}$ & $\begin{array}{l}2.9 \\
3.5 \\
3.6 \\
3.6 \\
3.7\end{array}$ & $\begin{array}{l}6.2 \\
5.9 \\
5.7 \\
5.9 \\
5.7\end{array}$ & $\begin{array}{l}491 \\
475 \\
448 \\
508 \\
403\end{array}$ & $\begin{array}{l}0 \\
0 \\
0 \\
0 \\
0\end{array}$ & $\begin{array}{l}403 \\
390 \\
367 \\
417 \\
331\end{array}$ & $\begin{array}{r}1200 \\
1100 \\
1100 \\
1000 \\
970\end{array}$ & $\begin{array}{l}130 \\
130 \\
120 \\
150 \\
160\end{array}$ \\
\hline $\begin{array}{r}91 \\
98 \\
100 \\
100 \\
100\end{array}$ & $\begin{array}{l}290 \\
270 \\
320 \\
300 \\
330\end{array}$ & $\begin{array}{l}3.9 \\
3.5 \\
4.2 \\
4.0 \\
4.2\end{array}$ & $\begin{array}{l}5.8 \\
5.6 \\
5.9 \\
6.2 \\
6.0\end{array}$ & $\begin{array}{r}400 \\
400 \\
430 \\
70 \\
430\end{array}$ & $\begin{array}{l}0 \\
0 \\
0 \\
0 \\
0\end{array}$ & $\begin{array}{r}328 \\
330 \\
350 \\
57 \\
350\end{array}$ & $\begin{array}{l}1000 \\
1000 \\
1100 \\
1100 \\
1100\end{array}$ & $\begin{array}{l}160 \\
180 \\
150 \\
150 \\
160\end{array}$ \\
\hline
\end{tabular}

NITRO- NITRO- PHOS-

GEN, GEN,AM- PHORUS, MANGA- CARBON,

AMMONIA MONIA + ORTHO, IRON, NESE, ORGANIC

DIS- ORGANIC DIS- DIS- DIS- DIS-

SOLVED DIS. SOLVED SOLVED SOLVED SOLVED

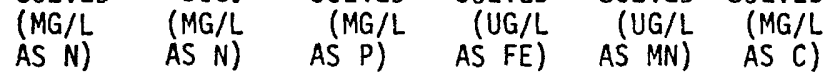

$\begin{array}{lccrrr}.06 & .75 & .03 & 120 & 260 & -- \\ .20 & .62 & .04 & 40 & 40 & -- \\ .07 & .83 & .06 & 20 & 0 & -- \\ -\overline{.04} & .01 & .07 & 50 & 0 & -- \\ & .01 & .11 & 20 & 20 & -- \\ .05 & .26 & .05 & 50 & 30 & -- \\ .02 & .37 & .04 & 20 & 0 & -- \\ .03 & .38 & .03 & 10 & 10 & -- \\ .01 & .66 & .03 & 10 & 10 & -- \\ .00 & .33 & .06 & 30 & 10 & -- \\ & & & & & \\ .01 & .32 & .03 & 10 & 10 & -- \\ .00 & .42 & .07 & 0 & 0 & 9.0 \\ .00 & .59 & .06 & 10 & 10 & 23 \\ .11 & .53 & .02 & 30 & 10 & -- \\ .01 & 1.6 & .05 & 30 & 10 & -- \\ & & & & & \\ .00 & .03 & .04 & 20 & 10 & -- \\ .01 & .67 & .01 & 30 & 0 & -- \\ .01 & .70 & .01 & 40 & 20 & -- \\ .01 & 1.0 & .01 & 10 & 0 & -- \\ .01 & 1.8 & .03 & 0 & 10 & --\end{array}$


Table 5.--Water-Zevel records and chemical [We11 number 26 on figure 2. Local we11 number is SB00506425ADD1.

\begin{tabular}{|c|c|c|c|c|c|c|c|c|}
\hline $\begin{array}{l}\text { DATE OF } \\
\text { WATER-LEVEL } \\
\text { MEASUREMENT }\end{array}$ & $\begin{array}{l}\text { WATER } \\
\text { LEVEL } \\
\text { (FEET } \\
\text { BELOW } \\
\text { LAND } \\
\text { SURFACE) }\end{array}$ & $\begin{array}{c}\text { DATE } \\
\text { OF } \\
\text { SAMPLE }\end{array}$ & $\begin{array}{l}\text { SPE- } \\
\text { CIFIC } \\
\text { CON- } \\
\text { DUCT- } \\
\text { ANCE } \\
\text { (MICRO- } \\
\text { MHOS) }\end{array}$ & $\begin{array}{c}\text { PH } \\
\text { (UNITS) }\end{array}$ & $\begin{array}{l}\text { TEMPER- } \\
\text { ATURE } \\
\text { (DEG C) }\end{array}$ & $\begin{array}{l}\text { HARD- } \\
\text { NESS } \\
\text { (MG/L } \\
\text { AS } \\
\text { CACO3) }\end{array}$ & $\begin{array}{l}\text { HARD- } \\
\text { NESS, } \\
\text { NONCAR- } \\
\text { BONATE } \\
\text { (MG/L } \\
\text { CAC03) }\end{array}$ & $\begin{array}{c}\text { CALCIUM, } \\
\text { DIS- } \\
\text { SOLVED } \\
\text { (MG/L } \\
\text { AS CA) }\end{array}$ \\
\hline $\begin{array}{l}74-04-04 \\
74-05-08 \\
74-06-14 \\
74-07-23 \\
74-08-27\end{array}$ & $\begin{array}{l}31.04 \\
31.20 \\
31.50 \\
32.40 \\
32.20\end{array}$ & $\begin{array}{l}74-04-04 \\
74-05-09 \\
74-06-14 \\
74-07-23 \\
74-08-27\end{array}$ & $\begin{array}{l}4330 \\
4330 \\
4310 \\
4510 \\
4550\end{array}$ & $\begin{array}{l}7.2 \\
7.3 \\
7.3 \\
7.4 \\
-.-\end{array}$ & $\begin{array}{l}12.5 \\
13.0 \\
13.5 \\
14.0 \\
13.5\end{array}$ & $\begin{array}{l}1500 \\
1600 \\
1500 \\
1600 \\
1600\end{array}$ & $\begin{array}{l}1100 \\
1200 \\
1100 \\
1200 \\
1200\end{array}$ & $\begin{array}{l}340 \\
340 \\
340 \\
340 \\
350\end{array}$ \\
\hline $\begin{array}{l}74-10-03 \\
74-11-11 \\
74-12-05 \\
75-02-06 \\
75-05-14\end{array}$ & $\begin{array}{l}30.60 \\
30.69 \\
30.90 \\
31.09 \\
31.75\end{array}$ & $\begin{array}{l}74-10-03 \\
74-11-11 \\
74-12-05 \\
75-02-06 \\
75-05-14\end{array}$ & $\begin{array}{l}4490 \\
4500 \\
5700 \\
5000 \\
6500\end{array}$ & $\begin{array}{l}-.- \\
7.2 \\
7.1 \\
7.0 \\
7.3\end{array}$ & $\begin{array}{l}13.5 \\
12.5 \\
13.0 \\
13.0 \\
13.5\end{array}$ & $\begin{array}{l}1500 \\
1500 \\
1500 \\
1500 \\
1500\end{array}$ & $\begin{array}{l}1100 \\
1100 \\
1100 \\
1100 \\
1100\end{array}$ & $\begin{array}{l}330 \\
320 \\
330 \\
320 \\
330\end{array}$ \\
\hline $\begin{array}{l}75-08-11 \\
75-12-01 \\
76-03-29 \\
76-06-29 \\
76-09-22\end{array}$ & $\begin{array}{l}32.32 \\
30.74 \\
31.15 \\
32.75 \\
31.24\end{array}$ & $\begin{array}{l}75-08-11 \\
75-12-01 \\
76-03-29 \\
76-06-29 \\
76-09-22\end{array}$ & $\begin{array}{l}3700 \\
5500 \\
4200 \\
4400 \\
4000\end{array}$ & $\begin{array}{l}7.6 \\
7.4 \\
7.4 \\
6.6 \\
7.1\end{array}$ & $\begin{array}{l}14.0 \\
12.5 \\
12.0 \\
13.5 \\
13.0\end{array}$ & $\begin{array}{l}1500 \\
1400 \\
1400 \\
1400 \\
1400\end{array}$ & $\begin{array}{l}1100 \\
1100 \\
1100 \\
1000 \\
1000\end{array}$ & $\begin{array}{l}310 \\
310 \\
330 \\
310 \\
320\end{array}$ \\
\hline $\begin{array}{l}76-12-08 \\
77-03-08 \\
77-06-29 \\
77-09-13 \\
77-12-06\end{array}$ & $\begin{array}{l}30.70 \\
31.20 \\
32.58 \\
31.57 \\
30.33\end{array}$ & $\begin{array}{l}76-12-08 \\
77-03-08 \\
77-06-15 \\
77-09-13 \\
77-12-06\end{array}$ & $\begin{array}{l}4000 \\
4500 \\
5000 \\
5000 \\
4000\end{array}$ & $\begin{array}{l}7.3 \\
7.6 \\
7.5 \\
7.3 \\
7.2\end{array}$ & $\begin{array}{l}12.5 \\
12.5 \\
13.5 \\
13.0 \\
12.0\end{array}$ & $\begin{array}{l}1400 \\
1400 \\
1500 \\
1400 \\
1400\end{array}$ & $\begin{array}{r}1100 \\
1100 \\
1100 \\
1000 \\
950\end{array}$ & $\begin{array}{l}310 \\
310 \\
320 \\
320 \\
300\end{array}$ \\
\hline $\begin{array}{l}78-03-09 \\
78-06-20\end{array}$ & $\begin{array}{l}31.06 \\
31.85\end{array}$ & $\begin{array}{l}78-03-09 \\
78-06-20\end{array}$ & $\begin{array}{l}4000 \\
4000\end{array}$ & $\begin{array}{l}7.2 \\
7.1\end{array}$ & $\begin{array}{l}13.0 \\
13.5\end{array}$ & $\begin{array}{l}1300 \\
1500\end{array}$ & $\begin{array}{l}1300 \\
1100\end{array}$ & \\
\hline $\begin{array}{l}\text { DATE OF } \\
\text { WATER-LEVEL } \\
\text { MEASUREMENT }\end{array}$ & $\begin{array}{l}\text { WATER } \\
\text { LEVEL } \\
\text { (FEET } \\
\text { BELOW } \\
\text { LAND } \\
\text { SURFACE) }\end{array}$ & $\begin{array}{c}\text { DATE } \\
\text { OF } \\
\text { SAMPLE }\end{array}$ & $\begin{array}{l}\text { FLUO- } \\
\text { RIDE, } \\
\text { DIS- } \\
\text { SOLVED } \\
\text { (MG/L } \\
\text { AS F) }\end{array}$ & $\begin{array}{l}\text { SILICA, } \\
\text { DIS- } \\
\text { SOLVED } \\
\text { (MG/L } \\
\text { AS } \\
\text { SI02) }\end{array}$ & $\begin{array}{l}\text { SOLIDS, } \\
\text { SUM OF } \\
\text { CONSTI- } \\
\text { TUENTS, } \\
\text { DIS- } \\
\text { SOLVED } \\
\text { (MG/L) }\end{array}$ & $\begin{array}{c}\text { NITRO- } \\
\text { GEN, } \\
\text { NITRATE } \\
\text { DIS- } \\
\text { SOLVED } \\
\text { (MG/L } \\
\text { AS N) }\end{array}$ & $\begin{array}{c}\text { NITRO- } \\
\text { GEN, } \\
\text { NITRITE } \\
\text { DIS- } \\
\text { SOLVED } \\
\text { (MG/L } \\
\text { AS N) }\end{array}$ & $\begin{array}{c}\text { NITRO- } \\
\text { GEN, } \\
\text { NO2+NO3 } \\
\text { DIS- } \\
\text { SOLVED } \\
(\text { MG/L } \\
\text { AS N) }\end{array}$ \\
\hline $\begin{array}{l}74-04-04 \\
74-05-08 \\
74-06-14 \\
74-07-23 \\
74-08-27\end{array}$ & $\begin{array}{l}31.04 \\
31.20 \\
31.50 \\
32.40 \\
32.20\end{array}$ & $\begin{array}{l}74-04-04 \\
74-05-09 \\
74-06-14 \\
74-07-23 \\
74-08-27\end{array}$ & $\begin{array}{l}1.8 \\
2.0 \\
2.5 \\
1.6 \\
1.7\end{array}$ & $\begin{array}{l}20 \\
20 \\
20 \\
21 \\
20\end{array}$ & $\begin{array}{l}3560 \\
3510 \\
3460 \\
3660 \\
3790\end{array}$ & $\begin{array}{c}5.1 \\
7.3 \\
.07 \\
10^{--}\end{array}$ & $\begin{array}{l}.06 \\
.04 \\
.01 \\
.- \\
.02\end{array}$ & $\begin{array}{l}5.2 \\
7.3 \\
.08 \\
8.3 \\
10\end{array}$ \\
\hline $\begin{array}{l}74-10-03 \\
74-11-11 \\
74-12-05 \\
75-02-06 \\
75-05-14\end{array}$ & $\begin{array}{l}30.60 \\
30.69 \\
30.90 \\
31.09 \\
31.75\end{array}$ & $\begin{array}{l}74-10-03 \\
74-11-11 \\
74-12-05 \\
75-02-06 \\
75-05-14\end{array}$ & $\begin{array}{l}1.8 \\
1.5 \\
1.7 \\
1.6 \\
1.8\end{array}$ & $\begin{array}{l}20 \\
20 \\
19 \\
20 \\
19\end{array}$ & $\begin{array}{l}3520 \\
3390 \\
3520 \\
3530 \\
3520\end{array}$ & $\begin{array}{l}8.6 \\
8.5 \\
5.9 \\
7.4 \\
8.1\end{array}$ & $\begin{array}{l}.01 \\
.00 \\
.00 \\
.00 \\
.00\end{array}$ & $\begin{array}{l}8.6 \\
8.5 \\
5.9 \\
7.4 \\
8.1\end{array}$ \\
\hline $\begin{array}{l}75-08-11 \\
75-12-01 \\
76-03-29 \\
76-06-29 \\
76-09-22\end{array}$ & $\begin{array}{l}32.32 \\
30.74 \\
31.15 \\
32.75 \\
31.24\end{array}$ & $\begin{array}{l}75-08-11 \\
75-12-01 \\
76-03-29 \\
76-06-29 \\
76-09-22\end{array}$ & $\begin{array}{l}1.7 \\
1.8 \\
1.8 \\
1.8 \\
1.9\end{array}$ & $\begin{array}{l}20 \\
19 \\
19 \\
18 \\
19\end{array}$ & $\begin{array}{l}3640 \\
3610 \\
3420 \\
3490 \\
3500\end{array}$ & $\begin{array}{l}8.4 \\
7.5 \\
5.0 \\
7.6 \\
8.2\end{array}$ & $\begin{array}{l}.00 \\
.01 \\
.00 \\
.00 \\
.01\end{array}$ & $\begin{array}{l}8.4 \\
7.5 \\
5.0 \\
7.6 \\
8.2\end{array}$ \\
\hline $\begin{array}{l}76-12-08 \\
77-03-08 \\
77-06-29 \\
77-09-13 \\
77-12-06\end{array}$ & $\begin{array}{l}30.70 \\
31.20 \\
32.58 \\
31.57 \\
30.33\end{array}$ & $\begin{array}{l}76-12-08 \\
77-03-08 \\
77-06-15 \\
77-09-13 \\
77-12-06\end{array}$ & $\begin{array}{l}1.8 \\
1.7 \\
1.8 \\
1.8 \\
1.7\end{array}$ & $\begin{array}{l}18 \\
21 \\
18 \\
20 \\
19\end{array}$ & $\begin{array}{l}3380 \\
3400 \\
3380 \\
3370 \\
3170\end{array}$ & $\begin{array}{l}4.9 \\
4.6 \\
5.6 \\
2.5\end{array}$ & $\begin{array}{l}. \overline{.00} \\
.00 \\
.00 \\
.01\end{array}$ & $\begin{array}{l}6.5 \\
4.9 \\
4.6 \\
5.6 \\
2.5\end{array}$ \\
\hline $\begin{array}{l}78-03-09 \\
78-06-20\end{array}$ & $\begin{array}{l}31.06 \\
31.85\end{array}$ & $\begin{array}{l}78-03-09 \\
78-06-20\end{array}$ & $\begin{array}{l}1.8 \\
1.7\end{array}$ & $\begin{array}{l}17 \\
16\end{array}$ & $\begin{array}{l}2880 \\
3130\end{array}$ & $\begin{array}{l}2.3 \\
3.6\end{array}$ & $\begin{array}{l}.01 \\
.03\end{array}$ & $\begin{array}{l}2.3 \\
3.6\end{array}$ \\
\hline
\end{tabular}


analyses of water from observation wells--Continued

Depth is $90 \mathrm{ft}$. Interval open to the aquifer is 60 to $90 \mathrm{ft}$ ]

\begin{tabular}{|c|c|c|c|c|c|c|c|c|}
\hline $\begin{array}{l}\text { MAGNE- } \\
\text { SIUM, } \\
\text { DIS- } \\
\text { SOLVED } \\
\text { (MG/L } \\
\text { AS MG) }\end{array}$ & $\begin{array}{l}\text { SODIUM, } \\
\text { DIS- } \\
\text { SOLVED } \\
\text { (MG/L } \\
\text { AS NA) }\end{array}$ & $\begin{array}{l}\text { SODIUM } \\
\text { AD- } \\
\text { SORP- } \\
\text { TION } \\
\text { RATIO }\end{array}$ & $\begin{array}{l}\text { POTAS- } \\
\text { SIUM, } \\
\text { DIS- } \\
\text { SOLVED } \\
\text { (MG/L } \\
\text { AS K) }\end{array}$ & $\begin{array}{c}\text { BICAR- } \\
\text { BONATE } \\
\text { (MG/L } \\
\text { AS } \\
\text { HCO3) }\end{array}$ & $\begin{array}{c}\text { CAR- } \\
\text { BONATE } \\
(M G / L \\
\text { AS CO3) }\end{array}$ & $\begin{array}{l}\text { ALKA- } \\
\text { LINITY } \\
\text { (MG/L } \\
\text { AS } \\
\text { CACO3) }\end{array}$ & $\begin{array}{c}\text { SULFATE, } \\
\text { DIS- } \\
\text { SOLVED } \\
\text { (MG/L } \\
\text { AS SO4) }\end{array}$ & $\begin{array}{l}\text { CHLO- } \\
\text { RIDE, } \\
\text { DIS- } \\
\text { SOLVED } \\
\text { (MG/L } \\
\text { AS CL) }\end{array}$ \\
\hline $\begin{array}{l}160 \\
170 \\
160 \\
170 \\
170\end{array}$ & $\begin{array}{l}600 \\
540 \\
550 \\
600 \\
600\end{array}$ & $\begin{array}{l}6.7 \\
6.0 \\
6.2 \\
6.6 \\
6.6\end{array}$ & $\begin{array}{r}10 \\
9.3 \\
9.5 \\
7.2 \\
11\end{array}$ & $\begin{array}{l}454 \\
445 \\
466 \\
481 \\
493\end{array}$ & $\begin{array}{r}0 \\
0 \\
0 \\
-- \\
--\end{array}$ & $\begin{array}{l}372 \\
365 \\
382 \\
395 \\
404\end{array}$ & $\begin{array}{l}2000 \\
2000 \\
2000 \\
2100 \\
2200\end{array}$ & $\begin{array}{l}180 \\
180 \\
150 \\
150 \\
150\end{array}$ \\
\hline $\begin{array}{l}160 \\
160 \\
160 \\
160 \\
160\end{array}$ & $\begin{array}{l}580 \\
580 \\
590 \\
580 \\
580\end{array}$ & $\begin{array}{l}6.6 \\
6.6 \\
6.7 \\
6.6 \\
6.6\end{array}$ & $\begin{array}{l}9.8 \\
5.2 \\
7.7 \\
9.5 \\
7.9\end{array}$ & $\begin{array}{l}484 \\
465 \\
462 \\
464 \\
466\end{array}$ & $\begin{array}{l}-- \\
-- \\
-- \\
-- \\
0\end{array}$ & $\begin{array}{l}397 \\
381 \\
379 \\
381 \\
382\end{array}$ & $\begin{array}{l}2000 \\
1900 \\
2000 \\
2000 \\
2000\end{array}$ & $\begin{array}{l}140 \\
140 \\
160 \\
180 \\
160\end{array}$ \\
\hline $\begin{array}{l}170 \\
160 \\
150 \\
160 \\
150\end{array}$ & $\begin{array}{l}600 \\
600 \\
560 \\
580 \\
590\end{array}$ & $\begin{array}{l}6.8 \\
6.9 \\
6.4 \\
6.7 \\
6.8\end{array}$ & $\begin{array}{c}10 \\
9.9 \\
10 \\
9.4 \\
9.6\end{array}$ & $\begin{array}{l}480 \\
463 \\
473 \\
469 \\
467\end{array}$ & $\begin{array}{l}0 \\
0 \\
0 \\
0 \\
0\end{array}$ & $\begin{array}{l}394 \\
380 \\
388 \\
385 \\
383\end{array}$ & $\begin{array}{l}2100 \\
2100 \\
1900 \\
2000 \\
2000\end{array}$ & $\begin{array}{l}150 \\
150 \\
190 \\
150 \\
140\end{array}$ \\
\hline $\begin{array}{l}160 \\
160 \\
170 \\
150 \\
150\end{array}$ & $\begin{array}{l}570 \\
550 \\
540 \\
540 \\
530\end{array}$ & $\begin{array}{l}6.6 \\
6.3 \\
6.1 \\
6.2 \\
6.2\end{array}$ & $\begin{array}{l}8.8 \\
8.5 \\
8.6 \\
8.6 \\
8.5\end{array}$ & $\begin{array}{l}458 \\
453 \\
450 \\
470 \\
510\end{array}$ & $\begin{array}{l}0 \\
0 \\
0 \\
0 \\
0\end{array}$ & $\begin{array}{l}376 \\
372 \\
369 \\
390 \\
420\end{array}$ & $\begin{array}{l}1900 \\
1900 \\
1900 \\
1900 \\
1700\end{array}$ & $\begin{array}{l}160 \\
200 \\
180 \\
170 \\
200\end{array}$ \\
\hline $\begin{array}{l}150 \\
170 \\
\text { NITRO- } \\
\text { GEN, } \\
\text { AMMONIA } \\
\text { DIS- } \\
\text { SOLVED } \\
\text { (MG/L } \\
\text { AS N) }\end{array}$ & $\begin{array}{l}480 \\
500 \\
\text { NITRO- } \\
\text { GEN,AM- } \\
\text { MONIA + } \\
\text { ORGANIC } \\
\text { DIS. } \\
\text { (MG/L } \\
\text { AS N) }\end{array}$ & $\begin{array}{r}5.7 \\
5.7 \\
\text { PHOS- } \\
\text { PHORUS } \\
\text { ORTHO, } \\
\text { DIS- } \\
\text { SOLVED } \\
\text { (MG/L } \\
\text { AS P) }\end{array}$ & $\begin{array}{l}\text { IRON, } \\
\text { DIS- } \\
\text { SOLVED } \\
\text { (UG/L } \\
\text { AS FE) }\end{array}$ & $\begin{array}{r}43 \\
470 \\
\text { MANGA- } \\
\text { NESE, } \\
\text { DIS- } \\
\text { SOLVED } \\
\text { (UG/L } \\
\text { AS MN) }\end{array}$ & $\begin{array}{r}0 \\
0 \\
\text { CARBON, } \\
\text { ORGANIC } \\
\text { DIS- } \\
\text { SOLVED } \\
\text { (MG/L } \\
\text { AS C) }\end{array}$ & $\begin{array}{r}35 \\
390\end{array}$ & $\begin{array}{l}1700 \\
1600\end{array}$ & $\begin{array}{l}200 \\
290\end{array}$ \\
\hline $\begin{array}{l}.05 \\
.08 \\
.06 \\
-. \\
.05\end{array}$ & $\begin{array}{r}.71 \\
.64 \\
.86 \\
.- \\
.67\end{array}$ & $\begin{array}{l}.03 \\
.04 \\
.06 \\
.07 \\
.11\end{array}$ & $\begin{array}{r}280 \\
30 \\
10 \\
20 \\
20\end{array}$ & $\begin{array}{r}130 \\
10 \\
0 \\
0 \\
0\end{array}$ & $\begin{array}{l}-- \\
-- \\
-- \\
-- \\
--\end{array}$ & & & \\
\hline $\begin{array}{l}.05 \\
.03 \\
.05 \\
.01 \\
.00\end{array}$ & $\begin{array}{l}.66 \\
.75 \\
.68 \\
.70 \\
.37\end{array}$ & $\begin{array}{l}.05 \\
.04 \\
.05 \\
.03 \\
.03\end{array}$ & $\begin{array}{l}40 \\
40 \\
20 \\
30 \\
50\end{array}$ & $\begin{array}{r}10 \\
0 \\
10 \\
0 \\
10\end{array}$ & $\begin{array}{l}-- \\
-- \\
-- \\
-- \\
--\end{array}$ & & & \\
\hline $\begin{array}{l}.00 \\
.02 \\
.02 \\
.00 \\
.00\end{array}$ & $\begin{array}{l}.67 \\
.96 \\
.88 \\
.46 \\
.55\end{array}$ & $\begin{array}{l}.06 \\
.05 \\
.02 \\
.06 \\
.03\end{array}$ & $\begin{array}{l}10 \\
50 \\
20 \\
20 \\
20\end{array}$ & $\begin{array}{r}0 \\
10 \\
0 \\
0 \\
10\end{array}$ & $\begin{array}{c}-- \\
26 \\
4.5\end{array}$ & & & \\
\hline $\begin{array}{l}.- \\
.00 \\
.00 \\
.00 \\
.01\end{array}$ & $\begin{array}{l}1 .-- \\
.47 \\
.70 \\
.36\end{array}$ & $\begin{array}{l}.04 \\
.05 \\
.04 \\
.02 \\
.02\end{array}$ & $\begin{array}{r}70 \\
90 \\
260 \\
60 \\
70\end{array}$ & $\begin{array}{l}10 \\
20 \\
20 \\
20 \\
10\end{array}$ & $\begin{array}{l}-- \\
-- \\
-- \\
-- \\
--\end{array}$ & & & \\
\hline $\begin{array}{l}.00 \\
.01\end{array}$ & $\begin{array}{l}.73 \\
.78\end{array}$ & $\begin{array}{l}.03 \\
.03\end{array}$ & $\begin{array}{l}50 \\
50\end{array}$ & $\begin{array}{l}20 \\
10\end{array}$ & $=$ & & & \\
\hline
\end{tabular}


Table 5.--Water-level records and chemical [We11 number 28 on figure 2. Local well number is SB00506425BBD1.

\begin{tabular}{|c|c|c|c|c|c|c|c|c|}
\hline $\begin{array}{l}\text { DATE OF } \\
\text { WATER-LEVEL } \\
\text { MEASUREMENT }\end{array}$ & $\begin{array}{l}\text { WATER } \\
\text { LEVEL } \\
\text { (FEET } \\
\text { BELOW } \\
\text { LAND } \\
\text { SURFACE) }\end{array}$ & $\begin{array}{c}\text { DATE } \\
\text { OF } \\
\text { SAMPLE }\end{array}$ & $\begin{array}{l}\text { FLUO- } \\
\text { RIDE, } \\
\text { DIS- } \\
\text { SOLVED } \\
\text { (MG/L } \\
\text { AS F) }\end{array}$ & $\begin{array}{l}\text { SILICA, } \\
\text { DIS- } \\
\text { SOLVED } \\
\text { (MG/L } \\
\text { AS } \\
\text { SI02) }\end{array}$ & $\begin{array}{l}\text { SOLIDS, } \\
\text { SUM OF } \\
\text { CONSTI- } \\
\text { TUENTS, } \\
\text { DIS- } \\
\text { SOLVED } \\
(M G / L)\end{array}$ & $\begin{array}{l}\text { NITRO- } \\
\text { GEN, } \\
\text { NITRATE } \\
\text { DIS- } \\
\text { SOLVED } \\
\text { (MG/L } \\
\text { AS N) }\end{array}$ & $\begin{array}{l}\text { NITRO- } \\
\text { GEN, } \\
\text { NITRITE } \\
\text { DIS- } \\
\text { SOLVED } \\
(M G / L \\
\text { AS N) }\end{array}$ & $\begin{array}{l}\text { NITRO- } \\
\text { GEN, } \\
\text { NO2+NO3 } \\
\text { DIS- } \\
\text { SOLVED } \\
\text { (MG/L } \\
\text { AS N) }\end{array}$ \\
\hline $\begin{array}{l}74-04-03 \\
74-05-08 \\
74-06-13 \\
74-07-23 \\
74-08-27\end{array}$ & $\begin{array}{l}35.86 \\
35.99 \\
35.86 \\
36.39 \\
36.32\end{array}$ & $\begin{array}{l}74-04-03 \\
74-05-08 \\
74-06-13 \\
74-07-23 \\
74-08-27\end{array}$ & $\begin{array}{l}1.3 \\
1.4 \\
1.2 \\
1.5 \\
1.5\end{array}$ & $\begin{array}{l}18 \\
19 \\
22 \\
19 \\
20\end{array}$ & $\begin{array}{l}1360 \\
1420 \\
1350 \\
1380 \\
1450\end{array}$ & $\begin{array}{l}3.7 \\
4.4 \\
5.0 \\
=- \\
=-\end{array}$ & $\begin{array}{l}.01 \\
.00 \\
.00 \\
.- \\
.01\end{array}$ & $\begin{array}{l}3.7 \\
4.4 \\
5.0 \\
4.5 \\
5.0\end{array}$ \\
\hline $\begin{array}{l}74-10-03 \\
74-11-11 \\
74-12-05 \\
75-02-06 \\
75-05-14\end{array}$ & $\begin{array}{l}35.82 \\
35.66 \\
35.73 \\
36.09 \\
36.38\end{array}$ & $\begin{array}{l}74-10-03 \\
74-11-11 \\
74-12-05 \\
75-02-06 \\
75-05-14\end{array}$ & $\begin{array}{l}1.3 \\
1.2 \\
1.4 \\
1.3 \\
1.7\end{array}$ & $\begin{array}{l}20 \\
19 \\
18 \\
20 \\
23\end{array}$ & $\begin{array}{l}1440 \\
1380 \\
1390 \\
1440 \\
1600\end{array}$ & $\begin{array}{l}4.5 \\
4.4 \\
4.8 \\
7.8\end{array}$ & $\begin{array}{l}.01 \\
.00 \\
.00 \\
.00 \\
.00\end{array}$ & $\begin{array}{l}5.0 \\
4.5 \\
4.4 \\
4.8 \\
7.8\end{array}$ \\
\hline $\begin{array}{l}75-08-11 \\
75-12-01 \\
76-03-29 \\
76-06-29 \\
76-09-22\end{array}$ & $\begin{array}{l}36.68 \\
35.75 \\
36.26 \\
37.20 \\
36.38\end{array}$ & $\begin{array}{l}75-08-11 \\
75-12-01 \\
76-03-29 \\
76-06-29 \\
76-09-22\end{array}$ & $\begin{array}{l}1.3 \\
1.3 \\
1.3 \\
1.4 \\
1.4\end{array}$ & $\begin{array}{l}18 \\
15 \\
18 \\
17 \\
17\end{array}$ & $\begin{array}{l}1430 \\
1430 \\
1400 \\
1420 \\
1510\end{array}$ & $\begin{array}{l}3.9 \\
3.5 \\
4.2 \\
4.2 \\
4.6\end{array}$ & $\begin{array}{l}.01 \\
.01 \\
.00 \\
.00 \\
.01\end{array}$ & $\begin{array}{l}3.9 \\
3.5 \\
4.2 \\
4.2 \\
4.6\end{array}$ \\
\hline $\begin{array}{l}76-12-08 \\
77-03-08 \\
77-06-15 \\
77-09-13 \\
77-12-06\end{array}$ & $\begin{array}{l}36.03 \\
36.36 \\
37.00 \\
37.18 \\
36.11\end{array}$ & $\begin{array}{l}76-12-08 \\
77-03-08 \\
77-06-15 \\
77-09-13 \\
77-12-06\end{array}$ & $\begin{array}{l}1.3 \\
1.5 \\
1.7 \\
1.4 \\
1.4\end{array}$ & $\begin{array}{l}17 \\
19 \\
22 \\
19 \\
18\end{array}$ & $\begin{array}{l}1500 \\
1500 \\
1490 \\
1520 \\
1530\end{array}$ & $\begin{array}{l}4.2 \\
3.8 \\
2.8 \\
3.7 \\
4.2\end{array}$ & $\begin{array}{l}.01 \\
.01 \\
.01 \\
.00 \\
.00\end{array}$ & $\begin{array}{l}4.2 \\
3.8 \\
2.8 \\
3.7 \\
4.2\end{array}$ \\
\hline $\begin{array}{l}78-03-09 \\
78-06-20\end{array}$ & $\begin{array}{l}36.49 \\
36.71\end{array}$ & $\begin{array}{l}78-03-09 \\
78-06-20\end{array}$ & $\begin{array}{l}1.4 \\
1.2\end{array}$ & $\begin{array}{l}17 \\
15\end{array}$ & $\begin{array}{l}1530 \\
1520\end{array}$ & $\begin{array}{l}2.9 \\
3.8\end{array}$ & $\begin{array}{l}.01 \\
.02\end{array}$ & $\begin{array}{l}2.9 \\
3.8\end{array}$ \\
\hline $\begin{array}{l}\text { DATE OF } \\
\text { WATER-LEVEL } \\
\text { MEASUREMENT }\end{array}$ & $\begin{array}{l}\text { WATER } \\
\text { LEVEL } \\
\text { (FEET } \\
\text { BELOW } \\
\text { LAND } \\
\text { SURFACE) }\end{array}$ & $\begin{array}{c}\text { DATE } \\
\text { OF } \\
\text { SAMPLE }\end{array}$ & $\begin{array}{l}\text { SPE- } \\
\text { CIFIC } \\
\text { CON- } \\
\text { DUCT- } \\
\text { ANCE } \\
\text { (MICRO- } \\
\text { MHOS) }\end{array}$ & $\begin{array}{c}\mathrm{PH} \\
\text { (UNITS) }\end{array}$ & $\begin{array}{l}\text { TEMPER- } \\
\text { ATURE } \\
\text { (DEG C) }\end{array}$ & $\begin{array}{l}\text { HARD- } \\
\text { NESS } \\
\text { (MG/L } \\
\text { AS } \\
\text { CACO3) }\end{array}$ & $\begin{array}{l}\text { HARD- } \\
\text { NESS, } \\
\text { NONCAR- } \\
\text { BONATE } \\
\text { (MG/L } \\
\text { CACO3) }\end{array}$ & $\begin{array}{l}\text { CALCIUM, } \\
\text { DIS- } \\
\text { SOLVED } \\
\text { (MG/L } \\
\text { AS CA) }\end{array}$ \\
\hline $\begin{array}{l}74-04-03 \\
74-05-08 \\
74-06-13 \\
74-07-23 \\
74-08-27\end{array}$ & $\begin{array}{l}35.86 \\
35.99 \\
35.86 \\
36.97 \\
36.32\end{array}$ & $\begin{array}{l}74-04-03 \\
74-05-08 \\
74-06-13 \\
74-07-23 \\
74-08-27\end{array}$ & $\begin{array}{l}1950 \\
1960 \\
1920 \\
1980 \\
2020\end{array}$ & $\begin{array}{l}7.4 \\
7.4 \\
7.5 \\
7.3 \\
--\end{array}$ & $\begin{array}{l}12.5 \\
14.0 \\
14.0 \\
14.5 \\
14.0\end{array}$ & $\begin{array}{l}750 \\
750 \\
750 \\
750 \\
800\end{array}$ & $\begin{array}{l}500 \\
490 \\
500 \\
500 \\
520\end{array}$ & $\begin{array}{l}180 \\
180 \\
180 \\
180 \\
190\end{array}$ \\
\hline $\begin{array}{l}74-10-03 \\
74-11-11 \\
74-12-05 \\
75-02-06 \\
75-05-14\end{array}$ & $\begin{array}{l}35.82 \\
35.66 \\
35.73 \\
36.09 \\
36.38\end{array}$ & $\begin{array}{l}74-10-03 \\
74-11-11 \\
74-12-05 \\
75-02-06 \\
75-05-14\end{array}$ & $\begin{array}{l}2000 \\
1950 \\
2100 \\
2100 \\
2700\end{array}$ & $\begin{array}{l}7 .-3 \\
7.2 \\
7.0 \\
7.0\end{array}$ & $\begin{array}{l}13.5 \\
13.0 \\
13.0 \\
13.0 \\
13.5\end{array}$ & $\begin{array}{l}750 \\
740 \\
770 \\
750 \\
810\end{array}$ & $\begin{array}{l}490 \\
480 \\
510 \\
480 \\
480\end{array}$ & $\begin{array}{l}180 \\
190 \\
190 \\
180 \\
210\end{array}$ \\
\hline $\begin{array}{l}75-08-11 \\
75-12-01 \\
76-03-29 \\
76-06-29 \\
76-09-22\end{array}$ & $\begin{array}{l}36.68 \\
35.75 \\
36.26 \\
37.20 \\
36.38\end{array}$ & $\begin{array}{l}75-08-11 \\
75-12-01 \\
76-03-29 \\
76-06-29 \\
76-09-22\end{array}$ & $\begin{array}{l}2400 \\
2300 \\
2000 \\
2000 \\
2000\end{array}$ & $\begin{array}{l}7.3 \\
7.3 \\
7.5 \\
6.7 \\
7.2\end{array}$ & $\begin{array}{l}14.0 \\
13.0 \\
13.0 \\
14.0 \\
13.5\end{array}$ & $\begin{array}{l}750 \\
780 \\
750 \\
750 \\
780\end{array}$ & $\begin{array}{l}500 \\
500 \\
490 \\
490 \\
540\end{array}$ & $\begin{array}{l}180 \\
190 \\
180 \\
180 \\
190\end{array}$ \\
\hline $\begin{array}{l}76-12-08 \\
77-03-08 \\
77-06-15 \\
77-09-13 \\
77-12-06\end{array}$ & $\begin{array}{l}36.03 \\
36.36 \\
37.00 \\
37.18 \\
36.11\end{array}$ & $\begin{array}{l}76-12-08 \\
77-03-08 \\
77-06-15 \\
77-09-13 \\
77-12-06\end{array}$ & $\begin{array}{l}1950 \\
2100 \\
2200 \\
2000 \\
2100\end{array}$ & $\begin{array}{l}7.4 \\
7.5 \\
7.2 \\
7.5 \\
7.3\end{array}$ & $\begin{array}{l}13.0 \\
13.5 \\
14.0 \\
13.5 \\
12.5\end{array}$ & $\begin{array}{l}790 \\
820 \\
810 \\
830 \\
820\end{array}$ & $\begin{array}{l}510 \\
530 \\
270 \\
500 \\
530\end{array}$ & $\begin{array}{l}190 \\
200 \\
190 \\
200 \\
200\end{array}$ \\
\hline $\begin{array}{l}78-03-09 \\
78-06-20\end{array}$ & $\begin{array}{l}36.49 \\
36.71\end{array}$ & $\begin{array}{l}78-03-09 \\
78-06-20\end{array}$ & $\begin{array}{l}2200 \\
2100\end{array}$ & $\begin{array}{l}7.3 \\
7.2\end{array}$ & $\begin{array}{l}13.5 \\
14.5\end{array}$ & $\begin{array}{l}880 \\
820\end{array}$ & $\begin{array}{l}540 \\
540\end{array}$ & $\begin{array}{l}210 \\
200\end{array}$ \\
\hline
\end{tabular}


analyses of water from observation wells--Continued

Depth is $103 \mathrm{ft}$. Interval open to the aquifer is 99 to $103 \mathrm{ft}$ ]

\begin{tabular}{|c|c|c|c|c|c|c|c|c|}
\hline $\begin{array}{l}\text { MAGNE- } \\
\text { SIUM, } \\
\text { DIS- } \\
\text { SOLVED } \\
\text { (MG/L } \\
\text { AS MG) }\end{array}$ & $\begin{array}{l}\text { SODIUM, } \\
\text { DIS- } \\
\text { SOLVED } \\
\text { (MG/L } \\
\text { AS NA) }\end{array}$ & $\begin{array}{c}\text { SODIUM } \\
\text { AD- } \\
\text { SORP- } \\
\text { TION } \\
\text { RATIO }\end{array}$ & $\begin{array}{l}\text { POTAS- } \\
\text { SIUM, } \\
\text { DIS- } \\
\text { SOLVED } \\
\text { (MG/L } \\
\text { AS K) }\end{array}$ & $\begin{array}{l}\text { BICAR- } \\
\text { BONATE } \\
\text { (MG/L } \\
\text { AS } \\
\text { HCO3) }\end{array}$ & $\begin{array}{c}\text { CAR- } \\
\text { BONATE } \\
\text { (MG/L } \\
\text { AS CO3) }\end{array}$ & $\begin{array}{c}\text { ALKA- } \\
\text { LINITY } \\
\text { (MG/L } \\
\text { AS } \\
\text { CACO3) }\end{array}$ & $\begin{array}{c}\text { SULFATE, } \\
\text { DIS- } \\
\text { SOLVED } \\
\text { (MG/L } \\
\text { AS SO4) }\end{array}$ & $\begin{array}{l}\text { CHLO- } \\
\text { RIDE, } \\
\text { DIS- } \\
\text { SOLVEL } \\
\text { (MG/L } \\
\text { AS CL }\end{array}$ \\
\hline $\begin{array}{l}72 \\
73 \\
72 \\
74 \\
79\end{array}$ & $\begin{array}{l}160 \\
160 \\
160 \\
170 \\
180\end{array}$ & $\begin{array}{l}2.6 \\
2.5 \\
2.6 \\
2.7 \\
2.8\end{array}$ & $\begin{array}{l}4.8 \\
5.4 \\
4.9 \\
3.3 \\
6.3\end{array}$ & $\begin{array}{l}302 \\
319 \\
304 \\
311 \\
336\end{array}$ & $\begin{array}{r}0 \\
0 \\
0 \\
-- \\
--\end{array}$ & $\begin{array}{l}248 \\
262 \\
249 \\
255 \\
276\end{array}$ & $\begin{array}{l}660 \\
700 \\
640 \\
660 \\
690\end{array}$ & $\begin{array}{r}97 \\
100 \\
93 \\
98 \\
100\end{array}$ \\
\hline $\begin{array}{l}74 \\
65 \\
72 \\
72 \\
70\end{array}$ & $\begin{array}{l}170 \\
180 \\
170 \\
180 \\
210\end{array}$ & $\begin{array}{l}2.7 \\
2.9 \\
2.7 \\
2.9 \\
3.2\end{array}$ & $\begin{array}{l}5.9 \\
3.6 \\
4.9 \\
5.6 \\
6.0\end{array}$ & $\begin{array}{l}323 \\
314 \\
317 \\
329 \\
406\end{array}$ & $\begin{array}{l}-- \\
-- \\
-- \\
0\end{array}$ & $\begin{array}{l}265 \\
258 \\
260 \\
270 \\
333\end{array}$ & $\begin{array}{l}710 \\
650 \\
660 \\
700 \\
750\end{array}$ & $\begin{array}{r}96 \\
93 \\
97 \\
100 \\
90\end{array}$ \\
\hline $\begin{array}{l}72 \\
73 \\
72 \\
73 \\
73\end{array}$ & $\begin{array}{l}170 \\
180 \\
170 \\
180 \\
180\end{array}$ & $\begin{array}{l}2.7 \\
2.8 \\
2.7 \\
2.9 \\
2.8\end{array}$ & $\begin{array}{l}5.5 \\
5.8 \\
5.4 \\
5.2 \\
5.2\end{array}$ & $\begin{array}{l}297 \\
341 \\
317 \\
319 \\
281\end{array}$ & $\begin{array}{l}0 \\
0 \\
0 \\
0 \\
0\end{array}$ & $\begin{array}{l}244 \\
280 \\
260 \\
262 \\
230\end{array}$ & $\begin{array}{l}710 \\
680 \\
670 \\
690 \\
760\end{array}$ & $\begin{array}{l}110 \\
100 \\
110 \\
100 \\
120\end{array}$ \\
\hline $\begin{array}{l}76 \\
77 \\
81 \\
80 \\
79\end{array}$ & $\begin{array}{l}180 \\
190 \\
200 \\
190 \\
190\end{array}$ & $\begin{array}{l}2.8 \\
2.9 \\
3.1 \\
2.9 \\
2.9\end{array}$ & $\begin{array}{l}5.3 \\
5.3 \\
5.7 \\
5.1 \\
5.3\end{array}$ & $\begin{array}{l}336 \\
347 \\
650 \\
400 \\
360\end{array}$ & $\begin{array}{l}0 \\
0 \\
0 \\
0 \\
0\end{array}$ & $\begin{array}{l}276 \\
285 \\
533 \\
330 \\
300\end{array}$ & $\begin{array}{l}730 \\
700 \\
560 \\
690 \\
720\end{array}$ & $\begin{array}{r}120 \\
120 \\
98 \\
120 \\
120\end{array}$ \\
\hline $\begin{array}{l}87 \\
79\end{array}$ & $\begin{array}{l}190 \\
190\end{array}$ & $\begin{array}{l}2.8 \\
2.9\end{array}$ & $\begin{array}{l}5.2 \\
5.3\end{array}$ & $\begin{array}{l}420 \\
350\end{array}$ & $\begin{array}{l}0 \\
0\end{array}$ & $\begin{array}{l}340 \\
290\end{array}$ & $\begin{array}{l}680 \\
710\end{array}$ & $\begin{array}{l}120 \\
130\end{array}$ \\
\hline
\end{tabular}

\begin{tabular}{|c|c|c|c|c|c|}
\hline $\begin{array}{l}\text { NITRO- } \\
\text { GEN, } \\
\text { AMMONIA } \\
\text { DIS- } \\
\text { SOLVED } \\
(M G / L \\
\text { AS N) }\end{array}$ & $\begin{array}{l}\text { NITRO- } \\
\text { GEN, AM- } \\
\text { MONIA + } \\
\text { ORGANIC } \\
\text { DIS. } \\
\text { (MG/L } \\
\text { AS N) }\end{array}$ & $\begin{array}{c}\text { PHOS- } \\
\text { PHORUS, } \\
\text { ORTHO, } \\
\text { DIS-- } \\
\text { SOL VED } \\
\text { (MG/L } \\
\text { AS P) }\end{array}$ & $\begin{array}{l}\text { IRON, } \\
\text { DIS- } \\
\text { SOLVED } \\
\text { (UG/L } \\
\text { AS FE) }\end{array}$ & $\begin{array}{l}\text { MANGA- } \\
\text { NESE, } \\
\text { DIS- } \\
\text { SOLVED } \\
\text { (UG/L } \\
\text { AS MN) }\end{array}$ & $\begin{array}{l}\text { CARBON, } \\
\text { ORGANIC } \\
\text { DIS- } \\
\text { SOLVED } \\
\text { (MG/L } \\
\text { AS C) }\end{array}$ \\
\hline $\begin{array}{l}.03 \\
.02 \\
.03 \\
.02 \\
.02\end{array}$ & $\begin{array}{l}.24 \\
.42 \\
.49 \\
. .43\end{array}$ & $\begin{array}{l}.02 \\
.00 \\
.01 \\
.06 \\
.04\end{array}$ & $\begin{array}{r}40 \\
0 \\
20 \\
30 \\
20\end{array}$ & $\begin{array}{r}20 \\
20 \\
20 \\
0 \\
20\end{array}$ & $\begin{array}{l}-- \\
-- \\
- \\
-\end{array}$ \\
\hline $\begin{array}{l}.03 \\
.04 \\
.04 \\
.00 \\
.00\end{array}$ & $\begin{array}{l}.55 \\
.41 \\
.29 \\
.35 \\
.19\end{array}$ & $\begin{array}{l}.02 \\
.01 \\
.03 \\
.04 \\
.06\end{array}$ & $\begin{array}{l}30 \\
30 \\
20 \\
20 \\
20\end{array}$ & $\begin{array}{r}10 \\
0 \\
10 \\
20 \\
5\end{array}$ & $\begin{array}{l}-- \\
-- \\
--\end{array}$ \\
\hline $\begin{array}{l}.00 \\
.02 \\
.02 \\
.00 \\
.00\end{array}$ & $\begin{array}{l}.25 \\
.65 \\
.38 \\
.16 \\
.30\end{array}$ & $\begin{array}{l}.02 \\
.03 \\
.01 \\
.03 \\
.03\end{array}$ & $\begin{array}{r}30 \\
150 \\
10 \\
40 \\
30\end{array}$ & $\begin{array}{r}0 \\
0 \\
0 \\
0 \\
10\end{array}$ & $\begin{array}{l}-- \\
35 \\
14\end{array}$ \\
\hline $\begin{array}{l}.00 \\
.01 \\
.00 \\
.00 \\
.01\end{array}$ & $\begin{array}{l}.38 \\
.25 \\
.33 \\
.42 \\
.16\end{array}$ & $\begin{array}{l}.01 \\
.04 \\
.04 \\
.01 \\
.01\end{array}$ & $\begin{array}{r}20 \\
60 \\
100 \\
120 \\
100\end{array}$ & $\begin{array}{r}0 \\
10 \\
20 \\
10 \\
20\end{array}$ & $\begin{array}{l}- \\
- \\
\ddot{-} \\
-\end{array}$ \\
\hline $\begin{array}{l}.00 \\
.01\end{array}$ & $\begin{array}{r}.62 \\
.49\end{array}$ & $\begin{array}{l}.01 \\
.02\end{array}$ & $\begin{array}{r}180 \\
80\end{array}$ & $\begin{array}{l}10 \\
10\end{array}$ & $=$ \\
\hline
\end{tabular}


Table 5.--Water-Zevel records and chemical

[We11 number 29 on figure 2. Local well number is SB00506425BBD2.

\begin{tabular}{|c|c|c|c|c|c|c|c|c|}
\hline $\begin{array}{l}\text { DATE OF } \\
\text { WATER-LEVEL } \\
\text { MEASUREMENT }\end{array}$ & $\begin{array}{l}\text { WATER } \\
\text { LEVEL } \\
\text { (FEET } \\
\text { BELOW } \\
\text { LAND } \\
\text { SURFACE) }\end{array}$ & $\begin{array}{l}\text { DATE } \\
\text { OF } \\
\text { SAMPLE }\end{array}$ & $\begin{array}{l}\text { SPE- } \\
\text { CIFIC } \\
\text { CON- } \\
\text { DUCT- } \\
\text { ANCE } \\
\text { (MICRO- } \\
\text { MHOS) }\end{array}$ & $\begin{array}{c}\text { PH } \\
\text { (UNITS) }\end{array}$ & $\begin{array}{l}\text { TEMPER- } \\
\text { ATURE } \\
\text { (DEG C) }\end{array}$ & $\begin{array}{l}\text { HARD- } \\
\text { NESS } \\
\text { (MG/L } \\
\text { AS } \\
\text { CACO3) }\end{array}$ & $\begin{array}{l}\text { HARD- } \\
\text { NESS, } \\
\text { NONCAR- } \\
\text { BONATE } \\
\text { (MG/L } \\
\text { CACO3) }\end{array}$ & $\begin{array}{l}\text { CALCIUM } \\
\text { DIS- } \\
\text { SOLVED } \\
\text { (MG/L } \\
\text { AS CA) }\end{array}$ \\
\hline $\begin{array}{l}74-04-03 \\
74-05-08 \\
74-06-13 \\
74-07-23 \\
74-08-27\end{array}$ & $\begin{array}{l}35.46 \\
35.56 \\
35.83 \\
36.07 \\
35.81\end{array}$ & $\begin{array}{l}74-04-03 \\
74-05-08 \\
74-06-13 \\
74-07-23 \\
74-08-27\end{array}$ & $\begin{array}{l}2130 \\
2150 \\
2200 \\
2150 \\
2130\end{array}$ & $\begin{array}{l}7.2 \\
7.4 \\
7.4 \\
7.3 \\
.-\end{array}$ & $\begin{array}{l}12.5 \\
14.0 \\
14.0 \\
14.5 \\
13.5\end{array}$ & $\begin{array}{l}850 \\
850 \\
860 \\
850 \\
780\end{array}$ & $\begin{array}{l}520 \\
520 \\
530 \\
520 \\
450\end{array}$ & $\begin{array}{l}200 \\
200 \\
200 \\
200 \\
190\end{array}$ \\
\hline $\begin{array}{l}74-10-03 \\
74-11-11 \\
74-12-05 \\
75-05-14 \\
75-08-11\end{array}$ & $\begin{array}{l}35.30 \\
35.26 \\
35.31 \\
38.55 \\
36.56\end{array}$ & $\begin{array}{l}74-10-03 \\
74-11-11 \\
74-12-05 \\
75-05-14 \\
75-08-11\end{array}$ & $\begin{array}{l}2110 \\
2150 \\
2300 \\
2400 \\
2700\end{array}$ & $\begin{array}{l}-.- \\
7.2 \\
7.1 \\
7.1 \\
7.3\end{array}$ & $\begin{array}{l}13.5 \\
12.5 \\
13.0 \\
14.0 \\
14.5\end{array}$ & $\begin{array}{l}820 \\
810 \\
850 \\
760 \\
820\end{array}$ & $\begin{array}{l}480 \\
470 \\
500 \\
510 \\
500\end{array}$ & $\begin{array}{l}190 \\
200 \\
200 \\
180 \\
190\end{array}$ \\
\hline $\begin{array}{l}75-12-01 \\
76-03-29 \\
76-06-29 \\
76-09-22 \\
76-12-08\end{array}$ & $\begin{array}{l}35.52 \\
36.04 \\
36.60 \\
36.26 \\
35.78\end{array}$ & $\begin{array}{l}75-12-01 \\
76-03-29 \\
76-06-29 \\
76-09-22 \\
76-12-08\end{array}$ & $\begin{array}{l}2300 \\
2000 \\
2100 \\
2000 \\
1900\end{array}$ & $\begin{array}{l}7.1 \\
7.5 \\
6.6 \\
7.0 \\
7.3\end{array}$ & $\begin{array}{l}13.0 \\
13.0 \\
13.0 \\
13.5 \\
13.0\end{array}$ & $\begin{array}{l}750 \\
750 \\
780 \\
800 \\
770\end{array}$ & $\begin{array}{l}370 \\
430 \\
340 \\
230 \\
350\end{array}$ & $\begin{array}{l}180 \\
180 \\
180 \\
190 \\
180\end{array}$ \\
\hline $\begin{array}{l}77-03-08 \\
77-06-15 \\
77-09-13 \\
77-12-06 \\
78-03-09\end{array}$ & $\begin{array}{l}36.21 \\
36.85 \\
37.04 \\
35.79 \\
36.35\end{array}$ & $\begin{array}{l}77-03-08 \\
77-06-15 \\
77-09-13 \\
77-12-06 \\
78-03-09\end{array}$ & $\begin{array}{l}2100 \\
2200 \\
2200 \\
2200 \\
2250\end{array}$ & $\begin{array}{l}7.5 \\
6.7 \\
7.3 \\
7.2 \\
7.2\end{array}$ & $\begin{array}{l}13.5 \\
13.5 \\
13.0 \\
12.5 \\
13.0\end{array}$ & $\begin{array}{l}770 \\
850 \\
900 \\
900 \\
920\end{array}$ & $\begin{array}{l}410 \\
270 \\
220 \\
180 \\
390\end{array}$ & $\begin{array}{l}180 \\
200 \\
210 \\
210 \\
220\end{array}$ \\
\hline $78-06-20$ & 36.40 & $78-06-20$ & 2400 & 7.2 & 14.0 & 1100 & 360 & 260 \\
\hline $\begin{array}{l}\text { DATE OF } \\
\text { WATER-LEVEL } \\
\text { MEASUREMENT }\end{array}$ & $\begin{array}{l}\text { WATER } \\
\text { LEVEL } \\
\text { (FEET } \\
\text { BELOW } \\
\text { LAND } \\
\text { SURFACE) }\end{array}$ & $\begin{array}{c}\text { DATE } \\
\text { OF } \\
\text { SAMPLE }\end{array}$ & $\begin{array}{l}\text { FLUO- } \\
\text { RIDE, } \\
\text { DIS- } \\
\text { SOLVED } \\
\text { (MG/L } \\
\text { AS F) }\end{array}$ & $\begin{array}{l}\text { SILICA, } \\
\text { DIS- } \\
\text { SOLVED } \\
\text { (MG/L } \\
\text { AS } \\
\text { SIO2) }\end{array}$ & $\begin{array}{c}\text { SOLIDS, } \\
\text { SUM OF } \\
\text { CONSTI- } \\
\text { TUENTS, } \\
\text { DIS- } \\
\text { SOLVED } \\
\text { (MG/L) }\end{array}$ & $\begin{array}{c}\text { NITRO- } \\
\text { GEN, } \\
\text { NITRATE } \\
\text { DIS- } \\
\text { SOLVED } \\
\text { (MG/L } \\
\text { AS N) }\end{array}$ & $\begin{array}{l}\text { NITRO- } \\
\text { GEN, } \\
\text { NITRITE } \\
\text { DIS- } \\
\text { SOLVED } \\
\text { (MG/L } \\
\text { AS N) }\end{array}$ & $\begin{array}{l}\text { NITRO- } \\
\text { GEN, } \\
\text { NO2+NO3 } \\
\text { DIS- } \\
\text { SOLVED } \\
\text { (MG/L } \\
\text { AS N) }\end{array}$ \\
\hline $\begin{array}{l}74-04-03 \\
74-05-08 \\
74-06-13 \\
74-07-23 \\
74-08-27\end{array}$ & $\begin{array}{l}35.46 \\
35.56 \\
35.83 \\
36.07 \\
35.81\end{array}$ & $\begin{array}{l}74-04-03 \\
74-05-08 \\
74-06-13 \\
74-07-23 \\
74-08-27\end{array}$ & $\begin{array}{l}1.7 \\
2.0 \\
2.1 \\
1.7 \\
1.7\end{array}$ & $\begin{array}{l}23 \\
23 \\
28 \\
24 \\
23\end{array}$ & $\begin{array}{l}1500 \\
1590 \\
1550 \\
1600 \\
1510\end{array}$ & $\begin{array}{l}7.2 \\
7.6 \\
7.0 \\
-- \\
--\end{array}$ & $\begin{array}{l}.02 \\
.01 \\
.01 \\
-. \\
.01\end{array}$ & $\begin{array}{c}7.2 \\
7.6 \\
7.0 \\
15 \\
7.6\end{array}$ \\
\hline $\begin{array}{l}74-10-03 \\
74-11-11 \\
74-12-05 \\
75-05-14 \\
75-08-11\end{array}$ & $\begin{array}{l}35.30 \\
35.26 \\
35.31 \\
38.55 \\
36.56\end{array}$ & $\begin{array}{l}74-10-03 \\
74-11-11 \\
74-12-05 \\
75-05-14 \\
75-08-11\end{array}$ & $\begin{array}{l}1.6 \\
1.4 \\
1.6 \\
1.3 \\
1.7\end{array}$ & $\begin{array}{l}24 \\
23 \\
23 \\
18 \\
23\end{array}$ & $\begin{array}{l}1550 \\
1500 \\
1540 \\
1400 \\
1540\end{array}$ & $\begin{array}{l}8.5 \\
7.8 \\
4.1 \\
7.2\end{array}$ & $\begin{array}{l}.01 \\
.00 \\
.00 \\
.00 \\
.01\end{array}$ & $\begin{array}{l}7.9 \\
8.5 \\
7.8 \\
4.1 \\
7.2\end{array}$ \\
\hline $\begin{array}{l}75-12-01 \\
76-03-29 \\
76-06-29 \\
76-09-22 \\
76-12-08\end{array}$ & $\begin{array}{l}35.52 \\
36.04 \\
36.60 \\
36.26 \\
35.78\end{array}$ & $\begin{array}{l}75-12-01 \\
76-03-29 \\
76-06-29 \\
76-09-22 \\
76-12-08\end{array}$ & $\begin{array}{l}1.2 \\
1.9 \\
1.7 \\
1.9 \\
1.8\end{array}$ & $\begin{array}{l}13 \\
22 \\
22 \\
23 \\
21\end{array}$ & $\begin{array}{l}1470 \\
1460 \\
1530 \\
1470 \\
1480\end{array}$ & $\begin{array}{l}3.8 \\
7.7 \\
5.2 \\
3.4 \\
9.0\end{array}$ & $\begin{array}{l}.00 \\
.00 \\
.01 \\
.02 \\
.00\end{array}$ & $\begin{array}{l}3.8 \\
7.7 \\
5.2 \\
3.4 \\
9.0\end{array}$ \\
\hline $\begin{array}{l}77-03-08 \\
77-06-15 \\
77-09-13 \\
77-12-06 \\
78-03-09\end{array}$ & $\begin{array}{l}36.21 \\
36.85 \\
37.04 \\
35.79 \\
36.35\end{array}$ & $\begin{array}{l}77-03-08 \\
77-06-15 \\
77-09-13 \\
77-12-06 \\
78-03-09\end{array}$ & $\begin{array}{l}1.9 \\
1.7 \\
1.9 \\
1.9 \\
2.0\end{array}$ & $\begin{array}{l}23 \\
23 \\
25 \\
25 \\
21\end{array}$ & $\begin{array}{l}1460 \\
1480 \\
1550 \\
1530 \\
1500\end{array}$ & $\begin{array}{l}6.3 \\
2.2 \\
.05 \\
.22 \\
.75\end{array}$ & $\begin{array}{l}.01 \\
.00 \\
.00 \\
.00 \\
.01\end{array}$ & $\begin{array}{l}6.3 \\
2.2 \\
.05 \\
.22 \\
.76\end{array}$ \\
\hline $78-06-20$ & 36.40 & $78-06-20$ & 2.0 & 20 & 1640 & .18 & .02 & .20 \\
\hline
\end{tabular}


analyses of water from observation wells--Continued

Depth is $68 \mathrm{ft}$. Interval open to the aquifer is 64 to $68 \mathrm{ft}$ ]

\begin{tabular}{|c|c|c|c|c|c|c|c|c|}
\hline $\begin{array}{l}\text { MAGNE- } \\
\text { SIUM, } \\
\text { DIS- } \\
\text { SOLVED } \\
\text { (MG/L } \\
\text { AS MG) }\end{array}$ & $\begin{array}{l}\text { SODIUM, } \\
\text { DIS- } \\
\text { SOLVED } \\
\text { (MG/L } \\
\text { AS NA) }\end{array}$ & $\begin{array}{l}\text { SODIUM } \\
\text { AD- } \\
\text { SORP- } \\
\text { TION } \\
\text { RATIO }\end{array}$ & $\begin{array}{l}\text { POTAS- } \\
\text { SIUM, } \\
\text { DIS- } \\
\text { SOLVED } \\
\text { (MG/L } \\
\text { AS K) }\end{array}$ & $\begin{array}{c}\text { BICAR- } \\
\text { BONATE } \\
\text { (MG/L } \\
\text { AS } \\
\text { HCO3) }\end{array}$ & $\begin{array}{c}\text { CAR- } \\
\text { BONATE } \\
\text { (MG/L } \\
\text { AS CO3) }\end{array}$ & $\begin{array}{c}\text { ALKA- } \\
\text { LINITY } \\
\text { (MG/L } \\
\text { AS } \\
\text { CACO3) }\end{array}$ & $\begin{array}{c}\text { SULFATE, } \\
\text { DIS- } \\
\text { SOLVED } \\
\text { (MG/L } \\
\text { AS SO4) }\end{array}$ & $\begin{array}{l}\text { CHLO- } \\
\text { RIDE, } \\
\text { DIS- } \\
\text { SOLVED } \\
\text { (MG/L } \\
\text { AS CL) }\end{array}$ \\
\hline $\begin{array}{l}85 \\
85 \\
87 \\
86 \\
75\end{array}$ & $\begin{array}{l}180 \\
180 \\
180 \\
190 \\
190\end{array}$ & $\begin{array}{l}2.7 \\
2.7 \\
2.7 \\
2.8 \\
3.0\end{array}$ & $\begin{array}{l}5.9 \\
6.3 \\
6.0 \\
6.0 \\
7.6\end{array}$ & $\begin{array}{l}405 \\
398 \\
395 \\
401 \\
406\end{array}$ & $\begin{array}{r}0 \\
0 \\
0 \\
-- \\
--\end{array}$ & $\begin{array}{l}332 \\
326 \\
324 \\
329 \\
333\end{array}$ & $\begin{array}{l}690 \\
770 \\
730 \\
730 \\
690\end{array}$ & $\begin{array}{l}86 \\
95 \\
89 \\
94 \\
96\end{array}$ \\
\hline $\begin{array}{l}83 \\
76 \\
84 \\
75 \\
83\end{array}$ & $\begin{array}{l}190 \\
200 \\
190 \\
170 \\
190\end{array}$ & $\begin{array}{l}2.9 \\
3.1 \\
2.8 \\
2.7 \\
2.9\end{array}$ & $\begin{array}{l}7.0 \\
4.1 \\
5.9 \\
4.7 \\
6.4\end{array}$ & $\begin{array}{l}410 \\
414 \\
421 \\
303 \\
387\end{array}$ & $\begin{array}{c}-- \\
-- \\
-- \\
0 \\
0\end{array}$ & $\begin{array}{l}336 \\
340 \\
345 \\
249 \\
317\end{array}$ & $\begin{array}{l}730 \\
670 \\
710 \\
670 \\
740\end{array}$ & $\begin{array}{r}89 \\
81 \\
86 \\
110 \\
86\end{array}$ \\
\hline $\begin{array}{l}73 \\
74 \\
81 \\
80 \\
77\end{array}$ & $\begin{array}{l}200 \\
190 \\
200 \\
200 \\
190\end{array}$ & $\begin{array}{l}3.2 \\
3.0 \\
3.1 \\
3.1 \\
3.0\end{array}$ & $\begin{array}{l}6.2 \\
5.9 \\
6.0 \\
6.0 \\
5.9\end{array}$ & $\begin{array}{l}468 \\
392 \\
538 \\
701 \\
509\end{array}$ & $\begin{array}{l}0 \\
0 \\
0 \\
0 \\
0\end{array}$ & $\begin{array}{l}384 \\
322 \\
441 \\
575 \\
417\end{array}$ & $\begin{array}{l}660 \\
670 \\
660 \\
520 \\
620\end{array}$ & $\begin{array}{l}85 \\
86 \\
89 \\
91 \\
92\end{array}$ \\
\hline $\begin{array}{l}78 \\
85 \\
92 \\
92 \\
89\end{array}$ & $\begin{array}{l}190 \\
200 \\
210 \\
200 \\
190\end{array}$ & $\begin{array}{l}3.0 \\
3.0 \\
3.0 \\
2.9 \\
2.7\end{array}$ & $\begin{array}{l}5.9 \\
5.9 \\
5.8 \\
6.2 \\
5.6\end{array}$ & $\begin{array}{l}439 \\
710 \\
830 \\
880 \\
640\end{array}$ & $\begin{array}{l}0 \\
0 \\
0 \\
0 \\
0\end{array}$ & $\begin{array}{l}360 \\
580 \\
680 \\
720 \\
530\end{array}$ & $\begin{array}{l}640 \\
510 \\
500 \\
470 \\
560\end{array}$ & $\begin{array}{r}100 \\
93 \\
100 \\
94 \\
96\end{array}$ \\
\hline 100 & 230 & 3.1 & 6.5 & 850 & 0 & 700 & 490 & 110 \\
\hline $\begin{array}{l}\text { NITRO- } \\
\text { GEN, } \\
\text { AMMONIA } \\
\text { DIS- } \\
\text { SOLVED } \\
\text { (MG/L } \\
\text { AS N) }\end{array}$ & $\begin{array}{l}\text { NITRO- } \\
\text { GEN,AM- } \\
\text { MONIA + } \\
\text { ORGANIC } \\
\text { DIS. } \\
(M G / L \\
\text { AS N) }\end{array}$ & $\begin{array}{l}\text { PHOS- } \\
\text { PHORUS, } \\
\text { ORTHO, } \\
\text { DIS- } \\
\text { SOLVED } \\
\text { (MG/L } \\
\text { AS P). }\end{array}$ & $\begin{array}{l}\text { IRON, } \\
\text { DIS- } \\
\text { SOLVED } \\
\text { (UG/L } \\
\text { AS FE) }\end{array}$ & $\begin{array}{l}\text { MANGA- } \\
\text { NESE, } \\
\text { DIS- } \\
\text { SOLVED } \\
\text { (UG/L } \\
\text { AS MN) }\end{array}$ & $\begin{array}{l}\text { CARBON, } \\
\text { ORGANIC } \\
\text { DIS- } \\
\text { SOLVED } \\
\text { (MG/L } \\
\text { AS C) }\end{array}$ & & & \\
\hline $\begin{array}{l}.07 \\
.08 \\
.01 \\
.- \\
.02\end{array}$ & $\begin{array}{l}.35 \\
.52 \\
.58 \\
. . \\
.42\end{array}$ & $\begin{array}{l}.03 \\
.02 \\
.02 \\
1.9 \\
.05\end{array}$ & $\begin{array}{l}80 \\
20 \\
10 \\
20 \\
20\end{array}$ & $\begin{array}{r}10 \\
10 \\
20 \\
0 \\
20\end{array}$ & $\begin{array}{l}-- \\
-- \\
-\infty \\
-\infty\end{array}$ & & & \\
\hline $\begin{array}{l}.04 \\
.04 \\
.07 \\
.00 \\
.00\end{array}$ & $\begin{array}{l}.38 \\
.58 \\
.30 \\
.16 \\
.34\end{array}$ & $\begin{array}{l}.04 \\
.03 \\
.04 \\
.02 \\
.04\end{array}$ & $\begin{array}{l}30 \\
10 \\
10 \\
30 \\
10\end{array}$ & $\begin{array}{r}10 \\
0 \\
10 \\
10 \\
0\end{array}$ & $\begin{array}{l}-- \\
-- \\
-- \\
--\end{array}$ & & & \\
\hline $\begin{array}{l}.05 \\
.02 \\
.00 \\
.00 \\
.00\end{array}$ & $\begin{array}{l}.67 \\
.46 \\
.21 \\
.32 \\
.85\end{array}$ & $\begin{array}{l}.03 \\
.02 \\
.06 \\
.06 \\
.03\end{array}$ & $\begin{array}{l}90 \\
10 \\
70 \\
60 \\
70\end{array}$ & $\begin{array}{r}0 \\
0 \\
0 \\
10 \\
10\end{array}$ & $\begin{array}{r}-- \\
-- \\
3.0 \\
3.2 \\
--\end{array}$ & & & \\
\hline $\begin{array}{l}.00 \\
.00 \\
.00 \\
.01 \\
.00\end{array}$ & $\begin{array}{l}.46 \\
.87 \\
.31 \\
.14 \\
.34\end{array}$ & $\begin{array}{l}.05 \\
.07 \\
.03 \\
.03 \\
.03\end{array}$ & $\begin{array}{r}50 \\
90 \\
200 \\
170 \\
70\end{array}$ & $\begin{array}{l}0 \\
10 \\
10 \\
10 \\
10\end{array}$ & $\begin{array}{l}-- \\
-- \\
-- \\
--\end{array}$ & & & \\
\hline .01 & .42 & .03 & 620 & 20 & - & & & \\
\hline
\end{tabular}


Table 5.--Water-Zevel records and chemical

[We11 number 30 on figure 2. Local wel1 number is SB00506425BBD3.

\begin{tabular}{|c|c|c|c|c|c|c|c|c|}
\hline $\begin{array}{l}\text { DATE OF } \\
\text { WATER-LEVEL } \\
\text { MEASUREMENT }\end{array}$ & $\begin{array}{l}\text { WATER } \\
\text { LEVEL } \\
\text { (FEET } \\
\text { BELOW } \\
\text { LAND } \\
\text { SURFACE) }\end{array}$ & $\begin{array}{c}\text { DATE } \\
\text { OF } \\
\text { SAMPLE }\end{array}$ & $\begin{array}{l}\text { SPE- } \\
\text { CIFIC } \\
\text { CON- } \\
\text { DUCT- } \\
\text { ANCE } \\
\text { (MICRO- } \\
\text { MHOS) }\end{array}$ & $\begin{array}{c}\text { PH } \\
\text { (UNITS) }\end{array}$ & $\begin{array}{l}\text { TEMPER- } \\
\text { ATURE } \\
\text { (DEG C) }\end{array}$ & $\begin{array}{l}\text { HARD- } \\
\text { NESS } \\
\text { (MG/L } \\
\text { AS } \\
\text { CACO3) }\end{array}$ & $\begin{array}{l}\text { HARD- } \\
\text { NESS, } \\
\text { NONCAR- } \\
\text { BONATE } \\
\text { (MG/L } \\
\text { CACO3) }\end{array}$ & $\begin{array}{c}\text { CALCIUM, } \\
\text { DIS- } \\
\text { SOLVED } \\
\text { (MG/L } \\
\text { AS CA) }\end{array}$ \\
\hline $\begin{array}{l}74-04-03 \\
74-05-08 \\
74-06-13 \\
74-07-23 \\
74-08-27\end{array}$ & $\begin{array}{l}35.47 \\
35.50 \\
35.83 \\
36.04 \\
35.77\end{array}$ & $\begin{array}{l}74-04-03 \\
74-05-08 \\
74-06-13 \\
74-07-23 \\
74-08-27\end{array}$ & $\begin{array}{l}2540 \\
2570 \\
2530 \\
2470 \\
2550\end{array}$ & $\begin{array}{l}7.4 \\
7.5 \\
7.5 \\
7.4 \\
7.2\end{array}$ & $\begin{array}{l}12.0 \\
13.0 \\
13.0 \\
13.5 \\
12.5\end{array}$ & $\begin{array}{l}950 \\
930 \\
890 \\
830 \\
800\end{array}$ & $\begin{array}{l}690 \\
650 \\
610 \\
560 \\
540\end{array}$ & $\begin{array}{l}200 \\
190 \\
190 \\
180 \\
190\end{array}$ \\
\hline $\begin{array}{l}74-10-03 \\
74-11-11 \\
74-12-05 \\
75-05-14 \\
75-08-11\end{array}$ & $\begin{array}{l}35.25 \\
36.34 \\
35.28 \\
37.54 \\
37.39\end{array}$ & $\begin{array}{l}74-10-03 \\
74-11-11 \\
74-12-05 \\
75-05-14 \\
75-08-11\end{array}$ & $\begin{array}{l}2570 \\
2500 \\
2900 \\
3500 \\
3200\end{array}$ & $\begin{array}{l}7 .- \\
7.3 \\
7.0 \\
7.1 \\
7.1\end{array}$ & $\begin{array}{l}12.5 \\
12.0 \\
12.5 \\
13.0 \\
13.0\end{array}$ & $\begin{array}{l}910 \\
900 \\
940 \\
940 \\
920\end{array}$ & $\begin{array}{l}610 \\
620 \\
670 \\
570 \\
600\end{array}$ & $\begin{array}{l}220 \\
220 \\
220 \\
220 \\
210\end{array}$ \\
\hline $\begin{array}{l}75-12-01 \\
76-03-29 \\
76-06-29 \\
76-09-22 \\
76-12-08\end{array}$ & $\begin{array}{l}35.47 \\
35.98 \\
36.58 \\
36.22 \\
36.38\end{array}$ & $\begin{array}{l}75-12-01 \\
76-03-29 \\
76-06-29 \\
76-09-22 \\
76-12-08\end{array}$ & $\begin{array}{l}3000 \\
2425 \\
2600 \\
2400 \\
2400\end{array}$ & $\begin{array}{l}7.1 \\
7.3 \\
6.6 \\
6.9 \\
7.2\end{array}$ & $\begin{array}{l}13.0 \\
13.0 \\
13.5 \\
13.5 \\
12.5\end{array}$ & $\begin{array}{l}910 \\
930 \\
900 \\
890 \\
910\end{array}$ & $\begin{array}{l}630 \\
630 \\
590 \\
580 \\
600\end{array}$ & $\begin{array}{l}210 \\
210 \\
180 \\
190 \\
200\end{array}$ \\
\hline $\begin{array}{l}77-03-08 \\
77-06-15 \\
77-09-13 \\
77-12-06 \\
78-03-09\end{array}$ & $\begin{array}{l}36.15 \\
37.28 \\
37.40 \\
35.95 \\
36.28\end{array}$ & $\begin{array}{l}77-03-08 \\
77-06-15 \\
77-09-13 \\
77-12-06 \\
78-03-09\end{array}$ & $\begin{array}{l}2500 \\
2450 \\
2400 \\
2400 \\
2400\end{array}$ & $\begin{array}{l}7.4 \\
7.3 \\
7.3 \\
7.4 \\
7.1\end{array}$ & $\begin{array}{l}13.5 \\
13.5 \\
13.0 \\
12.0 \\
13.0\end{array}$ & $\begin{array}{l}920 \\
900 \\
980 \\
940 \\
990\end{array}$ & $\begin{array}{l}590 \\
570 \\
600 \\
530 \\
500\end{array}$ & $\begin{array}{l}210 \\
210 \\
240 \\
230 \\
240\end{array}$ \\
\hline $78-06-20$ & 36.40 & $78-06-20$ & 2400 & 6.9 & 14.0 & 920 & 350 & 220 \\
\hline $\begin{array}{l}\text { DATE OF } \\
\text { WATER-LEVEL } \\
\text { MEASUREMENT }\end{array}$ & $\begin{array}{l}\text { WATER } \\
\text { LEVEL } \\
\text { (FEET } \\
\text { BELOW } \\
\text { LAND } \\
\text { SURFACE) }\end{array}$ & $\begin{array}{c}\text { DATE } \\
\text { OF } \\
\text { SAMPLE }\end{array}$ & $\begin{array}{l}\text { FLUO- } \\
\text { RIDE, } \\
\text { DIS- } \\
\text { SOLVED } \\
\text { (MG/L } \\
\text { AS F) }\end{array}$ & $\begin{array}{l}\text { SILICA, } \\
\text { DIS- } \\
\text { SOLVED } \\
\text { (MG/L } \\
\text { AS } \\
\text { SIO2) }\end{array}$ & $\begin{array}{l}\text { SOLIDS, } \\
\text { SUM OF } \\
\text { CONSTI- } \\
\text { TUENTS, } \\
\text { DIS- } \\
\text { SOLVED } \\
\text { (MG /L) }\end{array}$ & $\begin{array}{c}\text { NITRO- } \\
\text { GEN, } \\
\text { NITRATE } \\
\text { DIS- } \\
\text { SOLVED } \\
\text { (MG/L } \\
\text { AS N) }\end{array}$ & $\begin{array}{l}\text { NITRO- } \\
\text { GEN, } \\
\text { NITRITE } \\
\text { DIS- } \\
\text { SOLVED } \\
\text { (MG/L } \\
\text { AS N) }\end{array}$ & $\begin{array}{c}\text { NITRO- } \\
\text { GEN, } \\
\text { NO2+NO3 } \\
\text { DIS- } \\
\text { SOLVED } \\
(M G / L \\
\text { AS N) }\end{array}$ \\
\hline $\begin{array}{l}74-04-03 \\
74-05-08 \\
74-06-13 \\
74-07-23 \\
74-08-27\end{array}$ & $\begin{array}{l}35.47 \\
35.50 \\
35.83 \\
36.04 \\
35.77\end{array}$ & $\begin{array}{l}74-04-03 \\
74-05-08 \\
74-06-13 \\
74-07-23 \\
74-08-27\end{array}$ & $\begin{array}{r}2.1 \\
2.3 \\
2.6 \\
2.1 \\
.3\end{array}$ & $\begin{array}{l}24 \\
25 \\
29 \\
25 \\
7.2\end{array}$ & $\begin{array}{l}1810 \\
1900 \\
1830 \\
1810 \\
1720\end{array}$ & $\begin{array}{r}7.5 \\
8.2 \\
10 \\
. . \\
.17\end{array}$ & $\begin{array}{l}.04 \\
.01 \\
.00 \\
.01\end{array}$ & $\begin{array}{c}7.5 \\
8.2 \\
10 \\
6.6 \\
.18\end{array}$ \\
\hline $\begin{array}{l}74-10-03 \\
74-11-11 \\
74-12-05 \\
75-05-14 \\
75-08-11\end{array}$ & $\begin{array}{l}35.25 \\
36.34 \\
35.28 \\
37.54 \\
37.39\end{array}$ & $\begin{array}{l}74-10-03 \\
74-11-11 \\
74-12-05 \\
75-05-14 \\
75-08-11\end{array}$ & $\begin{array}{l}2.0 \\
1.9 \\
2.0 \\
2.0 \\
1.6\end{array}$ & $\begin{array}{l}25 \\
25 \\
24 \\
26 \\
29\end{array}$ & $\begin{array}{l}1930 \\
1790 \\
1940 \\
2030 \\
1950\end{array}$ & $\begin{array}{l}8 . \overline{6} \\
9.0 \\
8.4 \\
7.5\end{array}$ & $\begin{array}{l}.01 \\
.00 \\
.00 \\
.00 \\
.02\end{array}$ & $\begin{array}{l}8.3 \\
8.6 \\
9.0 \\
8.4 \\
7.5\end{array}$ \\
\hline $\begin{array}{l}75-12-01 \\
76-03-29 \\
76-06-29 \\
76-09-22 \\
76-12-08\end{array}$ & $\begin{array}{l}35.47 \\
35.98 \\
36.58 \\
36.22 \\
36.38\end{array}$ & $\begin{array}{l}75-12-01 \\
76-03-29 \\
76-06-29 \\
76-09-22 \\
76-12-08\end{array}$ & $\begin{array}{l}2.4 \\
2.5 \\
2.5 \\
2.3 \\
2.6\end{array}$ & $\begin{array}{l}27 \\
27 \\
27 \\
30 \\
27\end{array}$ & $\begin{array}{l}1920 \\
1940 \\
1870 \\
1860 \\
1880\end{array}$ & $\begin{array}{r}7.7 \\
8.4 \\
8.9 \\
9.3 \\
12\end{array}$ & $\begin{array}{l}.01 \\
.00 \\
.00 \\
.01 \\
.00\end{array}$ & $\begin{array}{r}7.7 \\
8.4 \\
8.9 \\
9.3 \\
12\end{array}$ \\
\hline $\begin{array}{l}77-03-08 \\
77-06-15 \\
77-09-13 \\
77-12-06 \\
78-03-09\end{array}$ & $\begin{array}{l}36.15 \\
37.28 \\
37.40 \\
35.95 \\
36.28\end{array}$ & $\begin{array}{l}77-03-08 \\
77-06-15 \\
77-09-13 \\
77-12-06 \\
78-03-09\end{array}$ & $\begin{array}{l}2.8 \\
2.8 \\
2.4 \\
2.7 \\
2.5\end{array}$ & $\begin{array}{l}27 \\
27 \\
30 \\
29 \\
27\end{array}$ & $\begin{array}{l}1820 \\
1780 \\
1850 \\
1800 \\
1800\end{array}$ & $\begin{array}{l}8.3 \\
9.3 \\
9.2 \\
9.3 \\
7.5\end{array}$ & $\begin{array}{l}.01 \\
.00 \\
.00 \\
.00 \\
.01\end{array}$ & $\begin{array}{l}8.3 \\
9.3 \\
9.2 \\
9.3 \\
7.5\end{array}$ \\
\hline $78-06-20$ & 36.40 & $78-06-20$ & 2.0 & 25 & 1720 & 5.7 & .05 & 5.7 \\
\hline
\end{tabular}


analyses of water from observation wells--Continued

Depth is $44 \mathrm{ft}$. Interval open to the aquifer is 40 to $44 \mathrm{ft}$ ]

\begin{tabular}{|c|c|c|c|c|c|c|c|c|}
\hline $\begin{array}{l}\text { MAGNE- } \\
\text { SIUM, } \\
\text { DIS- } \\
\text { SOLVED } \\
\text { (MG/L } \\
\text { AS MG) }\end{array}$ & $\begin{array}{l}\text { SODIUM, } \\
\text { DIS- } \\
\text { SOLVED } \\
\text { (MG/L } \\
\text { AS NA) }\end{array}$ & $\begin{array}{l}\text { SODIUM } \\
\text { AD- } \\
\text { SORP- } \\
\text { TION } \\
\text { RATIO }\end{array}$ & $\begin{array}{c}\text { POTAS- } \\
\text { SIUM, } \\
\text { DIS- } \\
\text { SOLVED } \\
\text { (MG/L } \\
\text { AS K) }\end{array}$ & $\begin{array}{l}\text { BICAR- } \\
\text { BONATE } \\
\text { (MG/L } \\
\text { AS } \\
\text { HCO3) }\end{array}$ & $\begin{array}{c}\text { CAR- } \\
\text { BONATE } \\
\text { (MG/L } \\
\text { AS C03) }\end{array}$ & $\begin{array}{c}\text { ALKA- } \\
\text { LINITY } \\
\text { (MG/L } \\
\text { AS } \\
\text { CACO3) }\end{array}$ & $\begin{array}{c}\text { SULFATE, } \\
\text { DIS- } \\
\text { SOLVED } \\
\text { (MG/L } \\
\text { AS SO4) }\end{array}$ & $\begin{array}{l}\text { CHLO- } \\
\text { RIDE, } \\
\text { DIS- } \\
\text { SOLVEL } \\
\text { (MG/L } \\
\text { AS CL }\end{array}$ \\
\hline $\begin{array}{r}110 \\
110 \\
100 \\
93 \\
80\end{array}$ & $\begin{array}{l}210 \\
250 \\
260 \\
270 \\
260\end{array}$ & $\begin{array}{l}3.0 \\
3.6 \\
3.8 \\
4.1 \\
4.0\end{array}$ & $\begin{array}{l}7.2 \\
7.8 \\
7.2 \\
7.3 \\
8.7\end{array}$ & $\begin{array}{l}318 \\
333 \\
337 \\
331 \\
319\end{array}$ & $\begin{array}{r}0 \\
0 \\
0 \\
-- \\
--\end{array}$ & $\begin{array}{l}261 \\
273 \\
276 \\
272 \\
262\end{array}$ & $\begin{array}{r}970 \\
1000 \\
940 \\
940 \\
920\end{array}$ & $\begin{array}{r}100 \\
110 \\
93 \\
95 \\
98\end{array}$ \\
\hline $\begin{array}{l}87 \\
85 \\
96 \\
96 \\
97\end{array}$ & $\begin{array}{l}260 \\
250 \\
280 \\
320 \\
280\end{array}$ & $\begin{array}{l}3.8 \\
3.6 \\
4.0 \\
4.5 \\
4.0\end{array}$ & $\begin{array}{l}8.2 \\
4.9 \\
6.8 \\
6.9 \\
8.1\end{array}$ & $\begin{array}{l}369 \\
341 \\
330 \\
457 \\
399\end{array}$ & $\begin{array}{l}-- \\
-- \\
-- \\
0 \\
0\end{array}$ & $\begin{array}{l}303 \\
280 \\
271 \\
375 \\
327\end{array}$ & $\begin{array}{r}1000 \\
900 \\
1000 \\
1000 \\
1000\end{array}$ & $\begin{array}{r}110 \\
100 \\
110 \\
100 \\
97\end{array}$ \\
\hline $\begin{array}{r}94 \\
99 \\
110 \\
100 \\
100\end{array}$ & $\begin{array}{l}280 \\
270 \\
290 \\
250 \\
240\end{array}$ & $\begin{array}{l}4.0 \\
3.8 \\
4.2 \\
3.7 \\
3.5\end{array}$ & $\begin{array}{l}7.9 \\
7.8 \\
7.5 \\
7.9 \\
7.7\end{array}$ & $\begin{array}{l}342 \\
372 \\
383 \\
373 \\
385\end{array}$ & $\begin{array}{l}0 \\
0 \\
0 \\
0 \\
0\end{array}$ & $\begin{array}{l}281 \\
305 \\
314 \\
306 \\
316\end{array}$ & $\begin{array}{r}1000 \\
1000 \\
930 \\
960 \\
970\end{array}$ & $\begin{array}{r}95 \\
100 \\
94 \\
93 \\
94\end{array}$ \\
\hline $\begin{array}{l}96 \\
90 \\
92 \\
88 \\
94\end{array}$ & $\begin{array}{l}250 \\
230 \\
230 \\
230 \\
220\end{array}$ & $\begin{array}{l}3.6 \\
3.3 \\
3.2 \\
3.3 \\
3.0\end{array}$ & $\begin{array}{l}7.2 \\
6.8 \\
6.3 \\
6.3 \\
5.6\end{array}$ & $\begin{array}{l}396 \\
400 \\
460 \\
490 \\
590\end{array}$ & $\begin{array}{l}0 \\
0 \\
0 \\
0 \\
0\end{array}$ & $\begin{array}{l}325 \\
328 \\
380 \\
400 \\
480\end{array}$ & $\begin{array}{l}900 \\
890 \\
890 \\
850 \\
800\end{array}$ & $\begin{array}{l}95 \\
86 \\
88 \\
80 \\
87\end{array}$ \\
\hline 90 & 230 & 3.3 & 5.8 & 690 & 0 & 570 & 700 & 86 \\
\hline
\end{tabular}

NITRO- NITRO- PHOS-

$\begin{array}{cccccc}\text { GEN, GEN,AM- } & \text { PHORUS, } & & \text { MANGA- } & \text { CARBON, } \\ \text { AMMONIA } & \text { MONIA + } & \text { ORTHO, } & \text { IRON, } & \text { NESE, } & \text { ORGANIC } \\ \text { OIS- } & \text { ORGANIC } & \text { DIS- } & \text { DIS- } & \text { DIS- } & \text { DIS- } \\ \text { SOLVED } & \text { DIS. } & \text { SOLVED } & \text { SOLVED } & \text { SOLVED } & \text { SOLVED } \\ \text { (MG/L } & \text { (MG/L } & \text { (MG/L } & \text { (UG/L } & \text { (UG/L } & \text { (MG/L } \\ \text { AS N) } & \text { AS N) } & \text { AS P) } & \text { AS FE) } & \text { AS MN) } & \text { AS C) }\end{array}$

$\begin{array}{lllrrr}.08 & .37 & .03 & 140 & 40 & -- \\ .06 & .43 & .02 & 50 & 10 & -- \\ .01 & .63 & .02 & 50 & 0 & -- \\ -- & -- & .08 & 30 & 0 & -- \\ .03 & .44 & .00 & 20 & 20 & -- \\ .02 & .33 & .03 & 50 & 0 & -- \\ .04 & .56 & .03 & 20 & 0 & -- \\ .04 & .28 & .04 & 20 & 10 & -- \\ .00 & .28 & .04 & 20 & 10 & -- \\ .00 & .32 & .04 & 50 & 0 & -- \\ .02 & .64 & .05 & 60 & 5 & -- \\ .02 & .44 & .02 & 0 & 10 & -- \\ .00 & .25 & .06 & 30 & 0 & 6.0 \\ .00 & .54 & .05 & 40 & 10 & 2.7 \\ .00 & .93 & .03 & 70 & 10 & -- \\ & & & & & \\ .00 & .50 & .06 & 70 & 0 & -- \\ .00 & .28 & .05 & 50 & 10 & -- \\ .00 & .36 & .02 & 60 & 0 & -- \\ .02 & .33 & .02 & 80 & 4 & -- \\ .00 & .51 & .02 & 50 & 10 & -- \\ .01 & .73 & .04 & 60 & 10 & --\end{array}$


Table 5.--Water-level records and chemical [We11 number 32 on figure 2. Local we11 number is SB00506425DBD1.

\begin{tabular}{|c|c|c|c|c|c|c|c|c|}
\hline $\begin{array}{l}\text { DATE OF } \\
\text { WATER-LEVEL } \\
\text { MEASUREMENT }\end{array}$ & $\begin{array}{l}\text { WATER } \\
\text { LEVEL } \\
\text { (FEET } \\
\text { BELOW } \\
\text { LAND } \\
\text { SURFACE) }\end{array}$ & $\begin{array}{c}\text { DATE } \\
\text { OF } \\
\text { SAMPLE }\end{array}$ & $\begin{array}{l}\text { SPE- } \\
\text { CIFIC } \\
\text { CON- } \\
\text { DUCT- } \\
\text { ANCE } \\
\text { (MICRO- } \\
\text { MHOS) }\end{array}$ & $\begin{array}{c}\text { PH } \\
\text { (UNITS) }\end{array}$ & $\begin{array}{l}\text { TEMPER- } \\
\text { ATURE } \\
\text { (DEG C) }\end{array}$ & $\begin{array}{l}\text { HARD- } \\
\text { NESS } \\
\text { (MG/L } \\
\text { AS } \\
\text { CACO3) }\end{array}$ & $\begin{array}{l}\text { HARD- } \\
\text { NESS, } \\
\text { NONCAR- } \\
\text { BONATE } \\
\text { (MG/L } \\
\text { CACO3) }\end{array}$ & $\begin{array}{l}\text { CALCIUM } \\
\text { DIS- } \\
\text { SOLVED } \\
\text { (MG/L } \\
\text { AS CA) }\end{array}$ \\
\hline $\begin{array}{l}74-04-04 \\
74-05-08 \\
74-06-14 \\
74-07-23 \\
74-08-27\end{array}$ & $\begin{array}{l}28.09 \\
28.17 \\
28.48 \\
28.08 \\
27.43\end{array}$ & $\begin{array}{l}74-04-04 \\
74-05-09 \\
74-06-14 \\
74-07-12 \\
74-08-27\end{array}$ & $\begin{array}{l}2560 \\
2700 \\
2740 \\
2690 \\
2620\end{array}$ & $\begin{array}{l}7.3 \\
7.3 \\
7.4 \\
7.4 \\
. .\end{array}$ & $\begin{array}{l}13.0 \\
13.5 \\
14.0 \\
14.5 \\
14.0\end{array}$ & $\begin{array}{r}990 \\
1100 \\
1000 \\
990 \\
940\end{array}$ & $\begin{array}{l}670 \\
730 \\
710 \\
670 \\
630\end{array}$ & $\begin{array}{l}230 \\
240 \\
250 \\
230 \\
210\end{array}$ \\
\hline $\begin{array}{l}74-10-03 \\
74-11-11 \\
74-12-05 \\
75-02-06 \\
75-05-14\end{array}$ & $\begin{array}{l}27.20 \\
27.37 \\
27.41 \\
27.85 \\
28.59\end{array}$ & $\begin{array}{l}74-10-03 \\
74-11-11 \\
74-12-05 \\
75-02-06 \\
75-05-14\end{array}$ & $\begin{array}{l}2590 \\
2550 \\
2800 \\
2650 \\
2700\end{array}$ & $\begin{array}{l}7 .- \\
7.0 \\
6.9 \\
7.0\end{array}$ & $\begin{array}{l}14.0 \\
13.5 \\
13.0 \\
13.0 \\
13.5\end{array}$ & $\begin{array}{l}940 \\
940 \\
950 \\
890 \\
840\end{array}$ & $\begin{array}{l}630 \\
650 \\
650 \\
570 \\
220\end{array}$ & $\begin{array}{l}210 \\
220 \\
220 \\
200 \\
200\end{array}$ \\
\hline $\begin{array}{l}75-08-11 \\
75-12-01 \\
76-03-29 \\
76-06-29 \\
76-09-22\end{array}$ & $\begin{array}{l}28.57 \\
27.54 \\
28.18 \\
.28 .97 \\
28.18\end{array}$ & $\begin{array}{l}75-08-11 \\
75-12-01 \\
76-03-29 \\
76-06-29 \\
76-09-22\end{array}$ & $\begin{array}{l}3100 \\
3000 \\
2450 \\
2700 \\
2800\end{array}$ & $\begin{array}{l}7.2 \\
7.2 \\
7.4 \\
6.4 \\
6.9\end{array}$ & $\begin{array}{l}14.5 \\
12.0 \\
11.0 \\
12.0 \\
11.5\end{array}$ & $\begin{array}{r}950 \\
960 \\
900 \\
990 \\
1100\end{array}$ & $\begin{array}{l}530 \\
600 \\
470 \\
360 \\
510\end{array}$ & $\begin{array}{l}220 \\
230 \\
210 \\
240 \\
270\end{array}$ \\
\hline $\begin{array}{l}76-12-08 \\
77-03-08 \\
77-06-15 \\
77-09-13 \\
77-12-06\end{array}$ & $\begin{array}{l}27.28 \\
28.23 \\
28.60 \\
28.49 \\
27.73\end{array}$ & $\begin{array}{l}76-12-08 \\
77-03-08 \\
77-06-15 \\
77-09-13 \\
77-12-06\end{array}$ & $\begin{array}{l}2600 \\
3000 \\
3100 \\
2800 \\
2600\end{array}$ & $\begin{array}{l}7.2 \\
7.5 \\
7.1 \\
7.1 \\
7.0\end{array}$ & $\begin{array}{l}11.0 \\
11.5 \\
12.0 \\
12.0 \\
11.0\end{array}$ & $\begin{array}{r}1000 \\
1100 \\
1100 \\
990 \\
960\end{array}$ & $\begin{array}{l}540 \\
480 \\
230 \\
450 \\
390\end{array}$ & $\begin{array}{l}240 \\
260 \\
260 \\
240 \\
220\end{array}$ \\
\hline $\begin{array}{l}78-03-09 \\
78-06-20\end{array}$ & $\begin{array}{l}27.80 \\
28.89\end{array}$ & $\begin{array}{l}78-03-09 \\
78-06-20\end{array}$ & $\begin{array}{l}3000 \\
2600\end{array}$ & $\begin{array}{l}7.0 \\
7.0\end{array}$ & $\begin{array}{l}11.5 \\
12.0\end{array}$ & $\begin{array}{r}1100 \\
960\end{array}$ & $\begin{array}{l}490 \\
110\end{array}$ & $\begin{array}{l}250 \\
220\end{array}$ \\
\hline $\begin{array}{c}\text { DATE OF } \\
\text { WATER-LEVEL } \\
\text { MEASUREMENT }\end{array}$ & $\begin{array}{l}\text { WATER } \\
\text { LEVEL } \\
\text { (FEET } \\
\text { BELOW } \\
\text { LAND } \\
\text { SURFACE) }\end{array}$ & $\begin{array}{c}\text { DATE } \\
\text { OF } \\
\text { SAMPLE }\end{array}$ & $\begin{array}{l}\text { FLUO- } \\
\text { RIDE, } \\
\text { DIS- } \\
\text { SOLVED } \\
\text { (MG/L } \\
\text { AS F) }\end{array}$ & $\begin{array}{l}\text { SILICA, } \\
\text { DIS- } \\
\text { SOLVED } \\
\text { (MG/L } \\
\text { AS } \\
\text { SI02) }\end{array}$ & $\begin{array}{c}\text { SOLIDS, } \\
\text { SUM OF } \\
\text { CONSTI- } \\
\text { TUENTS, } \\
\text { DIS-- } \\
\text { SOLVED } \\
\text { (MG/L) }\end{array}$ & $\begin{array}{l}\text { NITRO- } \\
\text { GEN, } \\
\text { NITRATE } \\
\text { DIS- } \\
\text { SOLVED } \\
(M G / L \\
\text { AS N) }\end{array}$ & $\begin{array}{c}\text { NITRO- } \\
\text { GEN, } \\
\text { NITRITE } \\
\text { DIS- } \\
\text { SOLVED } \\
\text { (MG/L } \\
\text { AS N) }\end{array}$ & $\begin{array}{l}\text { NITRO- } \\
\text { GEN, } \\
\text { NO2+NO3 } \\
\text { DIS- } \\
\text { SOLVED } \\
(M G / L \\
\text { AS N) }\end{array}$ \\
\hline $\begin{array}{l}74-04-04 \\
74-05-08 \\
74-06-14 \\
74-07-23 \\
74-08-27\end{array}$ & $\begin{array}{l}28.09 \\
28.17 \\
28.48 \\
28.08 \\
27.43\end{array}$ & $\begin{array}{l}74-04-04 \\
74-05-09 \\
74-06-14 \\
74-07-12 \\
74-08-27\end{array}$ & $\begin{array}{l}1.5 \\
1.5 \\
1.4 \\
1.5 \\
1.5\end{array}$ & $\begin{array}{l}17 \\
18 \\
18 \\
18 \\
18\end{array}$ & $\begin{array}{l}2040 \\
2060 \\
2090 \\
1950 \\
1890\end{array}$ & $\begin{array}{r}2.4 \\
3.2 \\
3.6 \\
-- \\
--\end{array}$ & $\begin{array}{l}.01 \\
.01 \\
.01 \\
.01\end{array}$ & $\begin{array}{l}2.4 \\
3.2 \\
3.6 \\
2.4 \\
2.2\end{array}$ \\
\hline $\begin{array}{l}74-10-03 \\
74-11-11 \\
74-12-05 \\
75-02-06 \\
75-05-14\end{array}$ & $\begin{array}{l}27.20 \\
27.37 \\
27.41 \\
27.85 \\
28.59\end{array}$ & $\begin{array}{l}74-10-03 \\
74-11-11 \\
74-12-05 \\
75-02-06 \\
75-05-14\end{array}$ & $\begin{array}{l}1.5 \\
1.3 \\
1.4 \\
1.3 \\
1.3\end{array}$ & $\begin{array}{l}18 \\
17 \\
17 \\
17 \\
19\end{array}$ & $\begin{array}{l}1870 \\
1850 \\
1850 \\
1790 \\
1450\end{array}$ & $\begin{array}{l}1.8 \\
1.7 \\
1.3 \\
4.6 \\
.14\end{array}$ & $\begin{array}{l}.00 \\
.00 \\
.00 \\
.00 \\
.01\end{array}$ & $\begin{array}{l}1.8 \\
1.7 \\
1.3 \\
4.6 \\
.15\end{array}$ \\
\hline $\begin{array}{l}75-08-11 \\
75-12-01 \\
76-03-29 \\
76-06-29 \\
76-09-22\end{array}$ & $\begin{array}{l}28.57 \\
27.54 \\
28.18 \\
28.97 \\
28.18\end{array}$ & $\begin{array}{l}75-08-11 \\
75-12-01 \\
76-03-29 \\
76-06-29 \\
76-09-22\end{array}$ & $\begin{array}{l}1.2 \\
1.3 \\
1.1 \\
1.2 \\
1.3\end{array}$ & $\begin{array}{l}19 \\
16 \\
16 \\
17 \\
18\end{array}$ & $\begin{array}{l}1730 \\
1950 \\
1800 \\
1930 \\
2210\end{array}$ & $\begin{array}{l}.26 \\
1.1 \\
1.3 \\
1.2 \\
3.0\end{array}$ & $\begin{array}{l}.01 \\
.04 \\
.01 \\
.02 \\
.03\end{array}$ & $\begin{array}{l}.27 \\
1.1 \\
1.3 \\
1.2 \\
3.0\end{array}$ \\
\hline $\begin{array}{l}76-12-08 \\
77-03-08 \\
77-06-15 \\
77-09-13 \\
77-12-06\end{array}$ & $\begin{array}{l}27.28 \\
28.23 \\
28.60 \\
28.49 \\
27.73\end{array}$ & $\begin{array}{l}76-12-08 \\
77-03-08 \\
77-06-15 \\
77-09-13 \\
77-12-06\end{array}$ & $\begin{array}{l}1.4 \\
1.5 \\
1.4 \\
1.4 \\
1.5\end{array}$ & $\begin{array}{l}18 \\
20 \\
21 \\
21 \\
21\end{array}$ & $\begin{array}{l}2070 \\
2080 \\
2080 \\
2070 \\
1900\end{array}$ & $\begin{array}{l}1.7 \\
.89 \\
.80 \\
2.3 \\
1.0\end{array}$ & $\begin{array}{l}.01 \\
.02 \\
.03 \\
.00 \\
.00\end{array}$ & $\begin{array}{l}1.7 \\
.91 \\
.83 \\
2.3 \\
1.0\end{array}$ \\
\hline $\begin{array}{l}78-03-09 \\
78-06-20\end{array}$ & $\begin{array}{l}27.80 \\
28.89\end{array}$ & $\begin{array}{l}78-03-09 \\
78-06-20\end{array}$ & $\begin{array}{l}1.4 \\
1.2\end{array}$ & $\begin{array}{l}21 \\
20\end{array}$ & $\begin{array}{l}1900 \\
1740\end{array}$ & $\begin{array}{l}.49 \\
.05\end{array}$ & $\begin{array}{l}.01 \\
.11\end{array}$ & $\begin{array}{l}.50 \\
.16\end{array}$ \\
\hline
\end{tabular}


analyses of water from observation wells--Continued

Depth is $68 \mathrm{ft}$. Interval open to the aquifer is 48 to $68 \mathrm{ft}$ ]

\begin{tabular}{|c|c|c|c|c|c|c|c|c|}
\hline $\begin{array}{l}\text { MAGNE- } \\
\text { SIUM, } \\
\text { DIS- } \\
\text { SOLVED } \\
\text { (MG/L } \\
\text { AS MG) }\end{array}$ & $\begin{array}{l}\text { SODIUM, } \\
\text { DIS- } \\
\text { SOLVED } \\
\text { (MG/L } \\
\text { AS NA) }\end{array}$ & $\begin{array}{c}\text { SODIUM } \\
\text { AD- } \\
\text { SORP- } \\
\text { TION } \\
\text { RATIO }\end{array}$ & $\begin{array}{l}\text { POTAS- } \\
\text { SIUM, } \\
\text { DIS- } \\
\text { SOLVED } \\
\text { (MG/L } \\
\text { AS K) }\end{array}$ & $\begin{array}{c}\text { BICAR- } \\
\text { BONATE } \\
\text { (MG/L } \\
\text { AS } \\
\mathrm{HCO} 3)\end{array}$ & $\begin{array}{c}\text { CAR- } \\
\text { BONATE } \\
\text { (MG/L } \\
\text { AS CO3) }\end{array}$ & $\begin{array}{c}\text { ALKA- } \\
\text { LINITY } \\
\text { (MG/L } \\
\text { AS } \\
\text { CAC03) }\end{array}$ & $\begin{array}{l}\text { SULFATE, } \\
\text { DIS- } \\
\text { SOLVED } \\
\text { (MG/L } \\
\text { AS SO4) }\end{array}$ & $\begin{array}{l}\text { CHLO- } \\
\text { RIDE, } \\
\text { DIS- } \\
\text { SOLVED } \\
\text { (MG/L } \\
\text { AS CL) }\end{array}$ \\
\hline $\begin{array}{l}100 \\
110 \\
100 \\
100 \\
100\end{array}$ & $\begin{array}{l}270 \\
250 \\
280 \\
270 \\
270\end{array}$ & $\begin{array}{l}3.7 \\
3.4 \\
3.8 \\
3.7 \\
3.8\end{array}$ & $\begin{array}{l}8.2 \\
8.3 \\
7.6 \\
5.5 \\
9.8\end{array}$ & $\begin{array}{l}381 \\
394 \\
400 \\
387 \\
368\end{array}$ & $\begin{array}{r}0 \\
0 \\
0 \\
-- \\
--\end{array}$ & $\begin{array}{l}313 \\
323 \\
328 \\
317 \\
302\end{array}$ & $\begin{array}{r}1100 \\
1100 \\
1100 \\
1000 \\
950\end{array}$ & $\begin{array}{l}110 \\
120 \\
120 \\
120 \\
140\end{array}$ \\
\hline $\begin{array}{r}100 \\
96 \\
98 \\
95 \\
82\end{array}$ & $\begin{array}{l}250 \\
270 \\
250 \\
250 \\
190\end{array}$ & $\begin{array}{l}3.6 \\
3.8 \\
3.5 \\
3.6 \\
2.9\end{array}$ & $\begin{array}{l}8.8 \\
5.4 \\
7.4 \\
8.3 \\
7.4\end{array}$ & $\begin{array}{l}369 \\
365 \\
372 \\
394 \\
747\end{array}$ & $\begin{array}{l}-- \\
-- \\
-- \\
0\end{array}$ & $\begin{array}{l}303 \\
299 \\
305 \\
323 \\
613\end{array}$ & $\begin{array}{l}950 \\
910 \\
940 \\
870 \\
450\end{array}$ & $\begin{array}{l}140 \\
140 \\
130 \\
130 \\
130\end{array}$ \\
\hline $\begin{array}{l}98 \\
94 \\
91 \\
95 \\
99\end{array}$ & $\begin{array}{l}210 \\
260 \\
250 \\
280 \\
310\end{array}$ & $\begin{array}{l}3.0 \\
3.7 \\
3.6 \\
3.9 \\
4.1\end{array}$ & $\begin{array}{l}8.5 \\
8.3 \\
8.5 \\
8.4 \\
8.9\end{array}$ & $\begin{array}{l}518 \\
444 \\
529 \\
771 \\
703\end{array}$ & $\begin{array}{l}0 \\
0 \\
0 \\
0 \\
0\end{array}$ & $\begin{array}{l}425 \\
364 \\
434 \\
632 \\
577\end{array}$ & $\begin{array}{r}800 \\
980 \\
830 \\
750 \\
1000\end{array}$ & $\begin{array}{l}120 \\
140 \\
130 \\
150 \\
140\end{array}$ \\
\hline $\begin{array}{r}100 \\
100 \\
110 \\
96 \\
100\end{array}$ & $\begin{array}{l}300 \\
310 \\
300 \\
340 \\
300\end{array}$ & $\begin{array}{l}4.1 \\
4.1 \\
3.9 \\
4.7 \\
4.2\end{array}$ & $\begin{array}{l}7.9 \\
7.9 \\
8.1 \\
7.4 \\
7.5\end{array}$ & $\begin{array}{r}577 \\
714 \\
1060 \\
660 \\
700\end{array}$ & $\begin{array}{l}0 \\
0 \\
0 \\
0 \\
0\end{array}$ & $\begin{array}{l}473 \\
586 \\
869 \\
540 \\
570\end{array}$ & $\begin{array}{l}970 \\
860 \\
690 \\
890 \\
760\end{array}$ & $\begin{array}{l}140 \\
160 \\
160 \\
140 \\
140\end{array}$ \\
\hline $\begin{array}{l}120 \\
100\end{array}$ & $\begin{array}{l}280 \\
270\end{array}$ & $\begin{array}{l}3.6 \\
3.8\end{array}$ & $\begin{array}{l}7.4 \\
7.9\end{array}$ & $\begin{array}{r}770 \\
1040\end{array}$ & $\begin{array}{l}0 \\
0\end{array}$ & $\begin{array}{l}630 \\
850\end{array}$ & $\begin{array}{l}670 \\
460\end{array}$ & $\begin{array}{l}160 \\
150\end{array}$ \\
\hline $\begin{array}{l}\text { NITRO- } \\
\text { GEN, } \\
\text { MMONIA } \\
\text { DIS- } \\
\text { SOLVED } \\
\text { (MG/L } \\
\text { AS N) }\end{array}$ & $\begin{array}{l}\text { NITRO- } \\
\text { GEN,AM- } \\
\text { MONIA + } \\
\text { ORGANIC } \\
\text { DIS. } \\
\text { (MG/L } \\
\text { AS N) }\end{array}$ & $\begin{array}{c}\text { PHOS- } \\
\text { PHORUS, } \\
\text { ORTHO, } \\
\text { DIS- } \\
\text { SOLVED } \\
\text { (MG/L } \\
\text { AS P) }\end{array}$ & $\begin{array}{l}\text { IRON, } \\
\text { DIS- } \\
\text { SOLVED } \\
\text { (UG/L } \\
\text { AS FE) }\end{array}$ & $\begin{array}{l}\text { MANGA- } \\
\text { NESE, } \\
\text { DIS- } \\
\text { SOLVED } \\
\text { (UG/L } \\
\text { AS MN) }\end{array}$ & $\begin{array}{l}\text { CARBON, } \\
\text { ORGANIC } \\
\text { DIS- } \\
\text { SOLVED } \\
\text { (MG/L } \\
\text { AS C) }\end{array}$ & & & \\
\hline $\begin{array}{l}.09 \\
.05 \\
.03 \\
. \\
.04\end{array}$ & $\begin{array}{l}.46 \\
.51 \\
.73 \\
. \overline{.47}\end{array}$ & $\begin{array}{l}.02 \\
.01 \\
.04 \\
.05 \\
.06\end{array}$ & $\begin{array}{r}590 \\
80 \\
20 \\
30 \\
20\end{array}$ & $\begin{array}{r}120 \\
20 \\
0 \\
0 \\
30\end{array}$ & $\begin{array}{l}-- \\
-- \\
-- \\
--\end{array}$ & & & \\
\hline $\begin{array}{l}.03 \\
.03 \\
.04 \\
.00 \\
.01\end{array}$ & $\begin{array}{l}.40 \\
.42 \\
.21 \\
.49 \\
1.0\end{array}$ & $\begin{array}{l}.02 \\
.01 \\
.03 \\
.01 \\
.03\end{array}$ & $\begin{array}{r}50 \\
30 \\
10 \\
40 \\
100\end{array}$ & $\begin{array}{r}30 \\
0 \\
10 \\
0 \\
50\end{array}$ & $\begin{array}{l}-- \\
-- \\
-- \\
--\end{array}$ & & & \\
\hline $\begin{array}{l}.00 \\
.01 \\
.03 \\
.00 \\
.01\end{array}$ & $\begin{array}{l}.62 \\
.77 \\
.69 \\
.95 \\
1.1\end{array}$ & $\begin{array}{l}.01 \\
.03 \\
.01 \\
.06 \\
.03\end{array}$ & $\begin{array}{r}70 \\
90 \\
140 \\
170 \\
180\end{array}$ & $\begin{array}{r}170 \\
170 \\
420 \\
1500 \\
2100\end{array}$ & $\begin{array}{l}-- \\
13 \\
21\end{array}$ & & & \\
\hline $\begin{array}{l}.00 \\
.02 \\
.00 \\
.0 \hat{1} 1 \\
.02\end{array}$ & $\begin{array}{c}.83 \\
.63 \\
1.1 \\
.61 \\
.59\end{array}$ & $\begin{array}{l}.01 \\
.05 \\
.03 \\
.02 \\
.02\end{array}$ & $\begin{array}{l}270 \\
230 \\
240 \\
320 \\
290\end{array}$ & $\begin{array}{l}1800 \\
2400 \\
3300 \\
2900 \\
2500\end{array}$ & $\begin{array}{l}-- \\
-- \\
-- \\
--\end{array}$ & & & \\
\hline $\begin{array}{l}.00 \\
.01\end{array}$ & 1.86 & $\begin{array}{l}.01 \\
.02\end{array}$ & $\begin{array}{l}740 \\
400\end{array}$ & $\begin{array}{l}3300 \\
1800\end{array}$ & -- & & & \\
\hline
\end{tabular}


Table 5.--Water-level records and chemical [Wel1 number 37 on figure 2. Local wel1 number is SB00506426ADD1.

\begin{tabular}{|c|c|c|c|c|c|c|c|c|}
\hline $\begin{array}{l}\text { DATE OF } \\
\text { WATER-LEVEL } \\
\text { MEASUREMENT }\end{array}$ & $\begin{array}{l}\text { WATER } \\
\text { LEVEL } \\
\text { (FEET } \\
\text { BELOW } \\
\text { LAND } \\
\text { SURFACE) }\end{array}$ & $\begin{array}{c}\text { DATE } \\
\text { OF } \\
\text { SAMPLE }\end{array}$ & $\begin{array}{l}\text { SPE- } \\
\text { CIFIC } \\
\text { CON- } \\
\text { DUCT- } \\
\text { ANCE- } \\
\text { (MICRO- } \\
\text { MHOS) }\end{array}$ & $\begin{array}{c}\text { PH } \\
\text { (UNITS) }\end{array}$ & $\begin{array}{l}\text { TEMPER- } \\
\text { ATURE } \\
\text { (DEG C) }\end{array}$ & $\begin{array}{l}\text { HARD- } \\
\text { NESS } \\
\text { (MG/L } \\
\text { AS } \\
\text { CACO3) }\end{array}$ & $\begin{array}{l}\text { HARD- } \\
\text { NESS, } \\
\text { NONCAR- } \\
\text { BONATE } \\
\text { (MG/L } \\
\text { CACO3) }\end{array}$ & $\begin{array}{l}\text { CALCIUM, } \\
\text { DIS- } \\
\text { SOLVED } \\
\text { (MG/L } \\
\text { AS CA) }\end{array}$ \\
\hline $\begin{array}{l}74-04-04 \\
74-05-08 \\
74-06-14 \\
74-07-23 \\
74-08-27\end{array}$ & $\begin{array}{l}26.67 \\
27.05 \\
27.19 \\
27.09 \\
25.74\end{array}$ & $\begin{array}{l}74-04-04 \\
74-05-08 \\
74-06-14 \\
74-07-24 \\
74-08-28\end{array}$ & $\begin{array}{l}2270 \\
2490 \\
2220 \\
2230 \\
2330\end{array}$ & $\begin{array}{l}7.1 \\
7.3 \\
7.4 \\
7.3 \\
-.\end{array}$ & $\begin{array}{l}12.5 \\
13.0 \\
13.5 \\
14.0 \\
13.0\end{array}$ & $\begin{array}{r}860 \\
1000 \\
860 \\
850 \\
940\end{array}$ & $\begin{array}{l}490 \\
630 \\
500 \\
480 \\
570\end{array}$ & $\begin{array}{l}200 \\
240 \\
200 \\
190 \\
220\end{array}$ \\
\hline $\begin{array}{l}74-10-03 \\
74-11-11 \\
74-12-05 \\
75-02-06 \\
75-05-14\end{array}$ & $\begin{array}{l}25.43 \\
25.73 \\
25.91 \\
26.59 \\
27.66\end{array}$ & $\begin{array}{l}74-10-04 \\
74-11-11 \\
74-12-05 \\
75-02-06 \\
75-05-14\end{array}$ & $\begin{array}{l}2270 \\
2450 \\
2650 \\
2700 \\
2800\end{array}$ & $\begin{array}{l}7 . \overline{1} \\
7.0 \\
6.8 \\
7.0\end{array}$ & $\begin{array}{l}12.5 \\
12.0 \\
13.0 \\
12.5 \\
13.5\end{array}$ & $\begin{array}{r}860 \\
930 \\
950 \\
1000 \\
850\end{array}$ & $\begin{array}{l}490 \\
560 \\
570 \\
610 \\
490\end{array}$ & $\begin{array}{l}200 \\
220 \\
220 \\
220 \\
190\end{array}$ \\
\hline $\begin{array}{l}75-08-12 \\
75-12-02 \\
76-03-29 \\
76-06-28 \\
76-09-22\end{array}$ & $\begin{array}{l}28.03 \\
25.99 \\
27.01 \\
27.87 \\
25.93\end{array}$ & $\begin{array}{l}75-08-12 \\
75-12-02 \\
76-03-29 \\
76-06-28 \\
76-09-22\end{array}$ & $\begin{array}{l}2600 \\
3200 \\
2400 \\
2100 \\
2200\end{array}$ & $\begin{array}{l}7.3 \\
7.3 \\
7.3 \\
7.1 \\
7.1\end{array}$ & $\begin{array}{l}13.5 \\
13.0 \\
12.0 \\
13.5 \\
13.0\end{array}$ & $\begin{array}{l}770 \\
970 \\
920 \\
720 \\
830\end{array}$ & $\begin{array}{l}410 \\
600 \\
530 \\
380 \\
480\end{array}$ & $\begin{array}{l}170 \\
230 \\
220 \\
160 \\
190\end{array}$ \\
\hline $\begin{array}{l}76-12-08 \\
77-03-08 \\
77-06-15 \\
77-09-13 \\
77-12-06\end{array}$ & $\begin{array}{l}26.18 \\
27.08 \\
27.89 \\
26.92 \\
26.62\end{array}$ & $\begin{array}{l}76-12-08 \\
77-03-08 \\
77-06-15 \\
77-09-12 \\
77-12-06\end{array}$ & $\begin{array}{l}2600 \\
3100 \\
2600 \\
2300 \\
2400\end{array}$ & $\begin{array}{l}7.3 \\
7.4 \\
7.5 \\
7.3 \\
7.3\end{array}$ & $\begin{array}{l}12.5 \\
13.0 \\
13.5 \\
13.0 \\
12.0\end{array}$ & $\begin{array}{r}1100 \\
1100 \\
880 \\
850 \\
900\end{array}$ & $\begin{array}{l}700 \\
700 \\
520 \\
480 \\
530\end{array}$ & $\begin{array}{l}250 \\
260 \\
200 \\
190 \\
200\end{array}$ \\
\hline $\begin{array}{l}78-03-09 \\
78-06-20\end{array}$ & $\begin{array}{l}27.16 \\
27.68\end{array}$ & $\begin{array}{l}78-03-09 \\
78-06-20\end{array}$ & $\begin{array}{l}2400 \\
2700\end{array}$ & $\begin{array}{l}7.2 \\
7.6\end{array}$ & $\begin{array}{l}13.0 \\
14.0\end{array}$ & $\begin{array}{r}900 \\
1100\end{array}$ & $\begin{array}{l}530 \\
750\end{array}$ & $\begin{array}{l}200 \\
260\end{array}$ \\
\hline $\begin{array}{l}\text { DATE OF } \\
\text { WATER-LEVEL } \\
\text { MEASUREMENT }\end{array}$ & $\begin{array}{l}\text { WATER } \\
\text { LEVEL } \\
\text { (FEET } \\
\text { BELOW } \\
\text { LAND } \\
\text { SURFACE) }\end{array}$ & $\begin{array}{c}\text { DATE } \\
\text { OF } \\
\text { SAMPLE }\end{array}$ & $\begin{array}{l}\text { FLUO- } \\
\text { RIDE, } \\
\text { DIS- } \\
\text { SOLVED } \\
\text { (MG/L } \\
\text { AS F) }\end{array}$ & $\begin{array}{l}\text { SILICA, } \\
\text { DIS- } \\
\text { SOLVED } \\
\text { (MG/L } \\
\text { AS } \\
\text { SI02) }\end{array}$ & $\begin{array}{l}\text { SOLIDS, } \\
\text { SUM OF } \\
\text { CONSTI- } \\
\text { TUENTS, } \\
\text { DIS- } \\
\text { SOLVED } \\
\text { (MG/L) }\end{array}$ & $\begin{array}{c}\text { NITRO- } \\
\text { GEN, } \\
\text { NITRATE } \\
\text { DIS- } \\
\text { SOLVED } \\
(\text { MG/L } \\
\text { AS N) }\end{array}$ & $\begin{array}{c}\text { NITRO- } \\
\text { GEN, } \\
\text { NITRITE } \\
\text { DIS- } \\
\text { SOLVED } \\
(M G / L \\
\text { AS N) }\end{array}$ & $\begin{array}{c}\text { NITRO- } \\
\text { GEN, } \\
\text { NO2+NO3 } \\
\text { DIS- } \\
\text { SOLVED } \\
\text { (MG/L } \\
\text { AS N) }\end{array}$ \\
\hline $\begin{array}{l}74-04-04 \\
74-05-08 \\
74-06-14 \\
74-07-23 \\
74-08-27\end{array}$ & $\begin{array}{l}26.67 \\
27.05 \\
27.19 \\
27.09 \\
25.74\end{array}$ & $\begin{array}{l}74-04-04 \\
74-05-08 \\
74-06-14 \\
74-07-24 \\
74-08-28\end{array}$ & $\begin{array}{l}2.0 \\
2.4 \\
2.5 \\
1.9 \\
2.0\end{array}$ & $\begin{array}{l}25 \\
26 \\
25 \\
26 \\
26\end{array}$ & $\begin{array}{l}1620 \\
1870 \\
1640 \\
1610 \\
1710\end{array}$ & $\begin{array}{r}5.7 \\
5.7 \\
7.4 \\
-. \\
--\end{array}$ & $\begin{array}{l}.31 \\
.02 \\
.01 \\
.01\end{array}$ & $\begin{array}{r}6.0 \\
5.7 \\
7.4 \\
7.3 \\
11\end{array}$ \\
\hline $\begin{array}{l}74-10-03 \\
74-11-11 \\
74-12-05 \\
75-02-06 \\
75-05-14\end{array}$ & $\begin{array}{l}25.43 \\
25.73 \\
25.91 \\
26.59 \\
27.66\end{array}$ & $\begin{array}{l}74-10-04 \\
74-11-11 \\
74-12-05 \\
75-02-06 \\
75-05-14\end{array}$ & $\begin{array}{l}1.9 \\
1.6 \\
1.7 \\
1.7 \\
1.9\end{array}$ & $\begin{array}{l}26 \\
25 \\
24 \\
26 \\
24\end{array}$ & $\begin{array}{l}1680 \\
1740 \\
1750 \\
1880 \\
1620\end{array}$ & $\begin{array}{l}12^{--} \\
10 \\
13 \\
8.7\end{array}$ & $\begin{array}{l}.01 \\
.00 \\
.00 \\
.00 \\
.00\end{array}$ & $\begin{array}{l}9.0 \\
12 \\
10 \\
13 \\
8.7\end{array}$ \\
\hline $\begin{array}{l}75-08-12 \\
75-12-02 \\
76-03-29 \\
76-06-28 \\
76-09-22\end{array}$ & $\begin{array}{l}28.03 \\
25.99 \\
27.01 \\
27.87 \\
25.93\end{array}$ & $\begin{array}{l}75-08-12 \\
75-12-02 \\
76-03-29 \\
76-06-28 \\
76-09-22\end{array}$ & $\begin{array}{l}1.8 \\
1.4 \\
1.8 \\
1.9 \\
1.9\end{array}$ & $\begin{array}{l}24 \\
18 \\
23 \\
23 \\
24\end{array}$ & $\begin{array}{l}1530 \\
1980 \\
1740 \\
1450 \\
1630\end{array}$ & $\begin{array}{l}6.9 \\
11 \\
12 \\
7.6 \\
9.8\end{array}$ & $\begin{array}{l}.01 \\
.01 \\
.00 \\
.00 \\
.01\end{array}$ & $\begin{array}{l}6.9 \\
11 \\
12 \\
7.6 \\
9.8\end{array}$ \\
\hline $\begin{array}{l}76-12-08 \\
77-03-08 \\
77-06-15 \\
77-09-13 \\
77-12-06\end{array}$ & $\begin{array}{l}26.18 \\
27.08 \\
27.89 \\
26.92 \\
26.62\end{array}$ & $\begin{array}{l}76-12-08 \\
77-03-08 \\
77-06-15 \\
77-09-12 \\
77-12-06\end{array}$ & $\begin{array}{l}1.9 \\
1.9 \\
1.8 \\
1.8 \\
1.9\end{array}$ & $\begin{array}{l}23 \\
25 \\
24 \\
26 \\
25\end{array}$ & $\begin{array}{l}2210 \\
2060 \\
1740 \\
1650 \\
1710\end{array}$ & $\begin{array}{l}20 \\
7.7 \\
11 \\
9.0 \\
11\end{array}$ & $\begin{array}{l}.00 \\
.01 \\
.00 \\
.00 \\
.01\end{array}$ & $\begin{array}{l}20 \\
7.7 \\
11 \\
9.0 \\
11\end{array}$ \\
\hline $\begin{array}{l}78-03-09 \\
78-06-20\end{array}$ & $\begin{array}{l}27.16 \\
27.68\end{array}$ & $\begin{array}{l}78-03-09 \\
78-06-20\end{array}$ & $\begin{array}{l}1.8 \\
1.7\end{array}$ & $\begin{array}{l}23 \\
21\end{array}$ & $\begin{array}{l}1630 \\
1980\end{array}$ & $\begin{array}{l}11 \\
30\end{array}$ & $\begin{array}{l}.00 \\
.03\end{array}$ & $\begin{array}{l}11 \\
30\end{array}$ \\
\hline
\end{tabular}


analyses of water from observation wells--Continued

Depth is $68 \mathrm{ft}$. Interval open to the aquifer is 64 to $68 \mathrm{ft}$ ]

\begin{tabular}{|c|c|c|c|c|c|c|c|c|}
\hline $\begin{array}{l}\text { MAGNE- } \\
\text { SIUM, } \\
\text { DIS- } \\
\text { SOLVED } \\
\text { (MG/L } \\
\text { AS MG) }\end{array}$ & $\begin{array}{l}\text { SODIUM, } \\
\text { DIS- } \\
\text { SOLVED } \\
\text { (MG/L } \\
\text { AS NA) }\end{array}$ & $\begin{array}{l}\text { SODIUM } \\
\text { AD- } \\
\text { SORP- } \\
\text { TION } \\
\text { RATIO }\end{array}$ & $\begin{array}{l}\text { POTAS- } \\
\text { SIUM, } \\
\text { DIS- } \\
\text { SOLVED } \\
\text { (MG/L } \\
\text { AS K) }\end{array}$ & $\begin{array}{l}\text { BICAR- } \\
\text { BONATE } \\
\text { (MG/L } \\
\text { AS } \\
\text { HCO3) }\end{array}$ & $\begin{array}{l}\text { CAR- } \\
\text { BONATE } \\
\text { (MG/L } \\
\text { AS CO3) }\end{array}$ & $\begin{array}{l}\text { ALKA- } \\
\text { LINITY } \\
\text { (MG/L } \\
\text { AS } \\
\text { CACO3) }\end{array}$ & $\begin{array}{c}\text { SULFATE, } \\
\text { DIS- } \\
\text { SOLVED } \\
\text { (MG/L } \\
\text { AS SO4) }\end{array}$ & $\begin{array}{l}\text { CHLO- } \\
\text { RIDE, } \\
\text { DIS- } \\
\text { SOLVED } \\
\text { (MG/L } \\
\text { AS CL) }\end{array}$ \\
\hline $\begin{array}{r}88 \\
100 \\
88 \\
91 \\
94\end{array}$ & $\begin{array}{l}220 \\
220 \\
210 \\
210 \\
210\end{array}$ & $\begin{array}{l}3.3 \\
3.0 \\
3.1 \\
3.1 \\
3.0\end{array}$ & $\begin{array}{l}6.6 \\
6.8 \\
6.1 \\
5.3 \\
7.8\end{array}$ & $\begin{array}{l}459 \\
460 \\
438 \\
446 \\
450\end{array}$ & $\begin{array}{r}0 \\
0 \\
0 \\
-- \\
--\end{array}$ & $\begin{array}{l}376 \\
377 \\
359 \\
366 \\
369\end{array}$ & $\begin{array}{l}730 \\
910 \\
770 \\
740 \\
780\end{array}$ & $\begin{array}{r}93 \\
110 \\
90 \\
94 \\
100\end{array}$ \\
\hline $\begin{array}{r}87 \\
93 \\
98 \\
110 \\
92\end{array}$ & $\begin{array}{l}210 \\
230 \\
220 \\
240 \\
220\end{array}$ & $\begin{array}{l}3.1 \\
3.3 \\
3.1 \\
3.3 \\
3.3\end{array}$ & $\begin{array}{l}6.6 \\
4.5 \\
6.0 \\
6.6 \\
5.4\end{array}$ & $\begin{array}{l}447 \\
458 \\
465 \\
478 \\
446\end{array}$ & $\begin{array}{l}-- \\
-- \\
-- \\
0\end{array}$ & $\begin{array}{l}367 \\
376 \\
381 \\
392 \\
366\end{array}$ & $\begin{array}{l}790 \\
780 \\
800 \\
850 \\
730\end{array}$ & $\begin{array}{r}96 \\
110 \\
110 \\
130 \\
100\end{array}$ \\
\hline $\begin{array}{l}84 \\
97 \\
90 \\
79 \\
87\end{array}$ & $\begin{array}{l}200 \\
260 \\
220 \\
200 \\
210\end{array}$ & $\begin{array}{l}3.1 \\
3.6 \\
3.2 \\
3.2 \\
3.2\end{array}$ & $\begin{array}{l}6.4 \\
7.4 \\
6.9 \\
5.8 \\
6.2\end{array}$ & $\begin{array}{l}438 \\
460 \\
472 \\
419 \\
436\end{array}$ & $\begin{array}{l}0 \\
0 \\
0 \\
0 \\
0\end{array}$ & $\begin{array}{l}359 \\
377 \\
387 \\
344 \\
358\end{array}$ & $\begin{array}{l}710 \\
960 \\
780 \\
650 \\
760\end{array}$ & $\begin{array}{r}85 \\
130 \\
110 \\
86 \\
91\end{array}$ \\
\hline $\begin{array}{r}110 \\
110 \\
93 \\
90 \\
97\end{array}$ & $\begin{array}{l}270 \\
270 \\
240 \\
220 \\
220\end{array}$ & $\begin{array}{l}3.6 \\
3.5 \\
3.5 \\
3.3 \\
3.2\end{array}$ & $\begin{array}{l}7.2 \\
7.1 \\
6.3 \\
5.8 \\
6.1\end{array}$ & $\begin{array}{l}455 \\
487 \\
440 \\
440 \\
450\end{array}$ & $\begin{array}{l}0 \\
0 \\
0 \\
0 \\
0\end{array}$ & $\begin{array}{l}373 \\
399 \\
361 \\
360 \\
370\end{array}$ & $\begin{array}{r}1100 \\
960 \\
800 \\
760 \\
790\end{array}$ & $\begin{array}{r}130 \\
150 \\
110 \\
96 \\
99\end{array}$ \\
\hline $\begin{array}{r}98 \\
110\end{array}$ & $\begin{array}{l}200 \\
240\end{array}$ & $\begin{array}{l}2.9 \\
3.1\end{array}$ & $\begin{array}{l}6.4 \\
6.9\end{array}$ & $\begin{array}{l}450 \\
430\end{array}$ & $\begin{array}{l}0 \\
0\end{array}$ & $\begin{array}{l}370 \\
350\end{array}$ & $\begin{array}{l}790 \\
830\end{array}$ & $\begin{array}{r}43 \\
170\end{array}$ \\
\hline $\begin{array}{l}\text { NITRO- } \\
\text { GEN, } \\
\text { AMMONIA } \\
\text { DIS- } \\
\text { SOLVED } \\
\text { (MG/L } \\
\text { AS N) }\end{array}$ & $\begin{array}{l}\text { NITRO- } \\
\text { GEN,AM- } \\
\text { MONIA + } \\
\text { ORGANIC } \\
\text { DIS. } \\
(M G / L \\
\text { AS N) }\end{array}$ & $\begin{array}{l}\text { PHOS- } \\
\text { PHORUS, } \\
\text { ORTHO, } \\
\text { DIS- } \\
\text { SOLVED } \\
\text { (MG/L } \\
\text { AS P) }\end{array}$ & $\begin{array}{l}\text { IRON, } \\
\text { DIS- } \\
\text { SOLVED } \\
\text { (UG/L } \\
\text { AS FE) }\end{array}$ & $\begin{array}{l}\text { MANGA- } \\
\text { NESE, } \\
\text { DIS- } \\
\text { SOL VED } \\
\text { (UG/L } \\
\text { AS MN) }\end{array}$ & $\begin{array}{l}\text { CARBON, } \\
\text { ORGANIC } \\
\text { DIS- } \\
\text { SOLVED } \\
\text { (MG/L } \\
\text { AS C) }\end{array}$ & & & \\
\hline $\begin{array}{l}.05 \\
.08 \\
.03 \\
.03\end{array}$ & $\begin{array}{r}1.2 \\
.62 \\
.72 \\
-. \\
.63\end{array}$ & $\begin{array}{l}.03 \\
.01 \\
.05 \\
.06 \\
.07\end{array}$ & $\begin{array}{r}40 \\
20 \\
0 \\
50 \\
60\end{array}$ & $\begin{array}{r}210 \\
110 \\
0 \\
0 \\
0\end{array}$ & $\begin{array}{l}- \\
-\infty \\
-\infty \\
-\infty\end{array}$ & & & \\
\hline $\begin{array}{l}.03 \\
.03 \\
.03 \\
.01 \\
.00\end{array}$ & $\begin{array}{l}.44 \\
.49 \\
.34 \\
.45 \\
.21\end{array}$ & $\begin{array}{l}.02 \\
.03 \\
.05 \\
.03 \\
.04\end{array}$ & $\begin{array}{l}50 \\
40 \\
10 \\
10 \\
20\end{array}$ & $\begin{array}{r}0 \\
0 \\
10 \\
0 \\
5\end{array}$ & $\begin{array}{l}-- \\
-- \\
-- \\
--\end{array}$ & & & \\
\hline $\begin{array}{l}.00 \\
.01 \\
.02 \\
.00 \\
.00\end{array}$ & $\begin{array}{l}.16 \\
.81 \\
.29 \\
.33 \\
.41\end{array}$ & $\begin{array}{l}.03 \\
.03 \\
.01 \\
.05 \\
.04\end{array}$ & $\begin{array}{l}60 \\
10 \\
20 \\
40 \\
30\end{array}$ & $\begin{array}{r}0 \\
5 \\
10 \\
0 \\
10\end{array}$ & $\begin{array}{l}-- \\
-- \\
-- \\
2.8 \\
3.0\end{array}$ & & & \\
\hline $\begin{array}{l}.00 \\
.00 \\
.00 \\
.01 \\
.00\end{array}$ & $\begin{array}{l}.75 \\
.66 \\
.65 \\
.55 \\
.05\end{array}$ & $\begin{array}{l}.02 \\
.05 \\
.04 \\
.02 \\
.03\end{array}$ & $\begin{array}{l}30 \\
70 \\
30 \\
30 \\
50\end{array}$ & $\begin{array}{r}10 \\
10 \\
10 \\
10 \\
8\end{array}$ & $\begin{array}{l}-- \\
-- \\
-- \\
--\end{array}$ & & & \\
\hline $\begin{array}{l}.00 \\
.01\end{array}$ & $\stackrel{.62}{1.9}$ & $\begin{array}{l}.04 \\
.02\end{array}$ & $\begin{array}{r}10 \\
0\end{array}$ & $\begin{array}{r}0 \\
10\end{array}$ & -- & & & \\
\hline
\end{tabular}





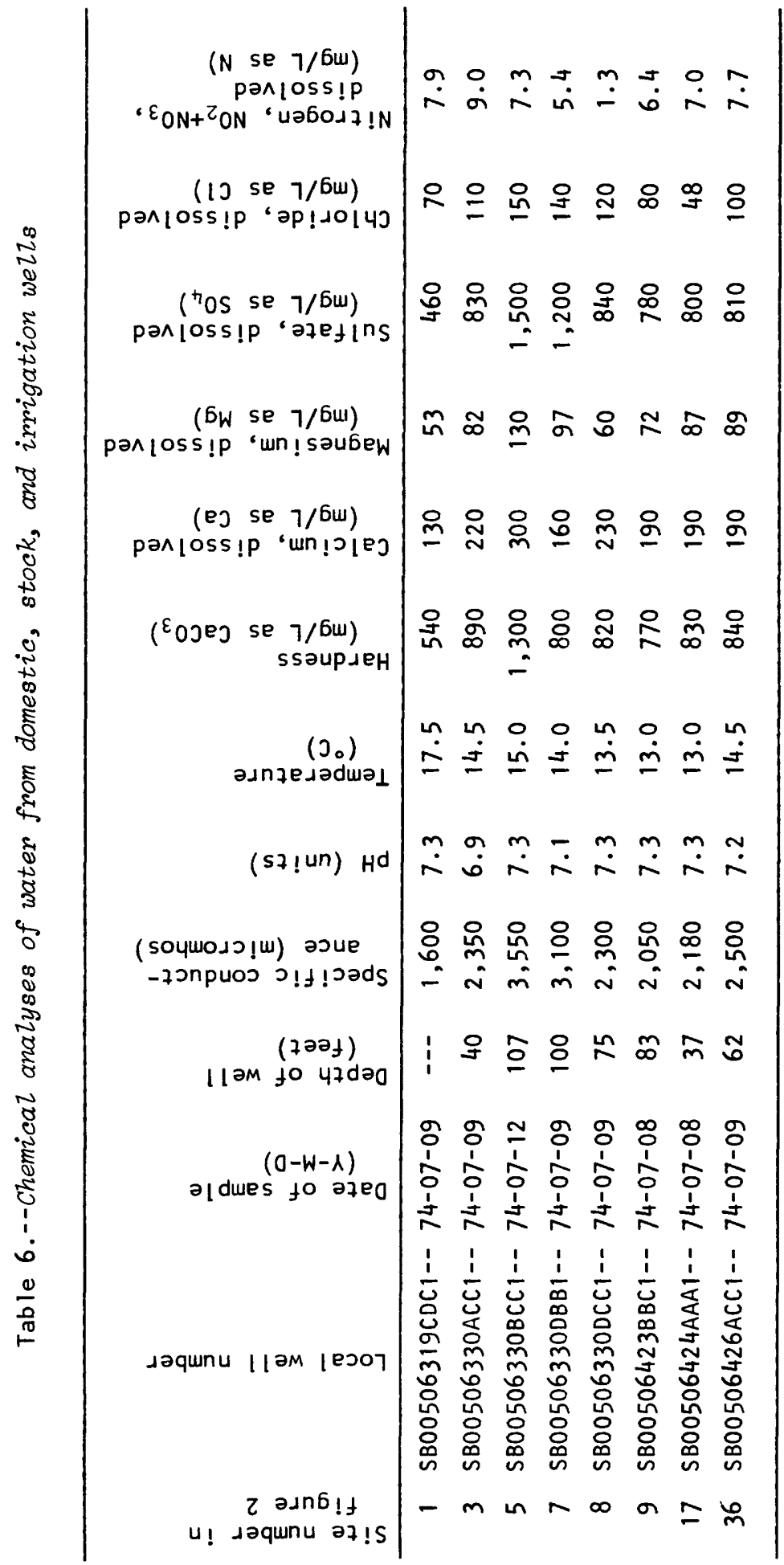




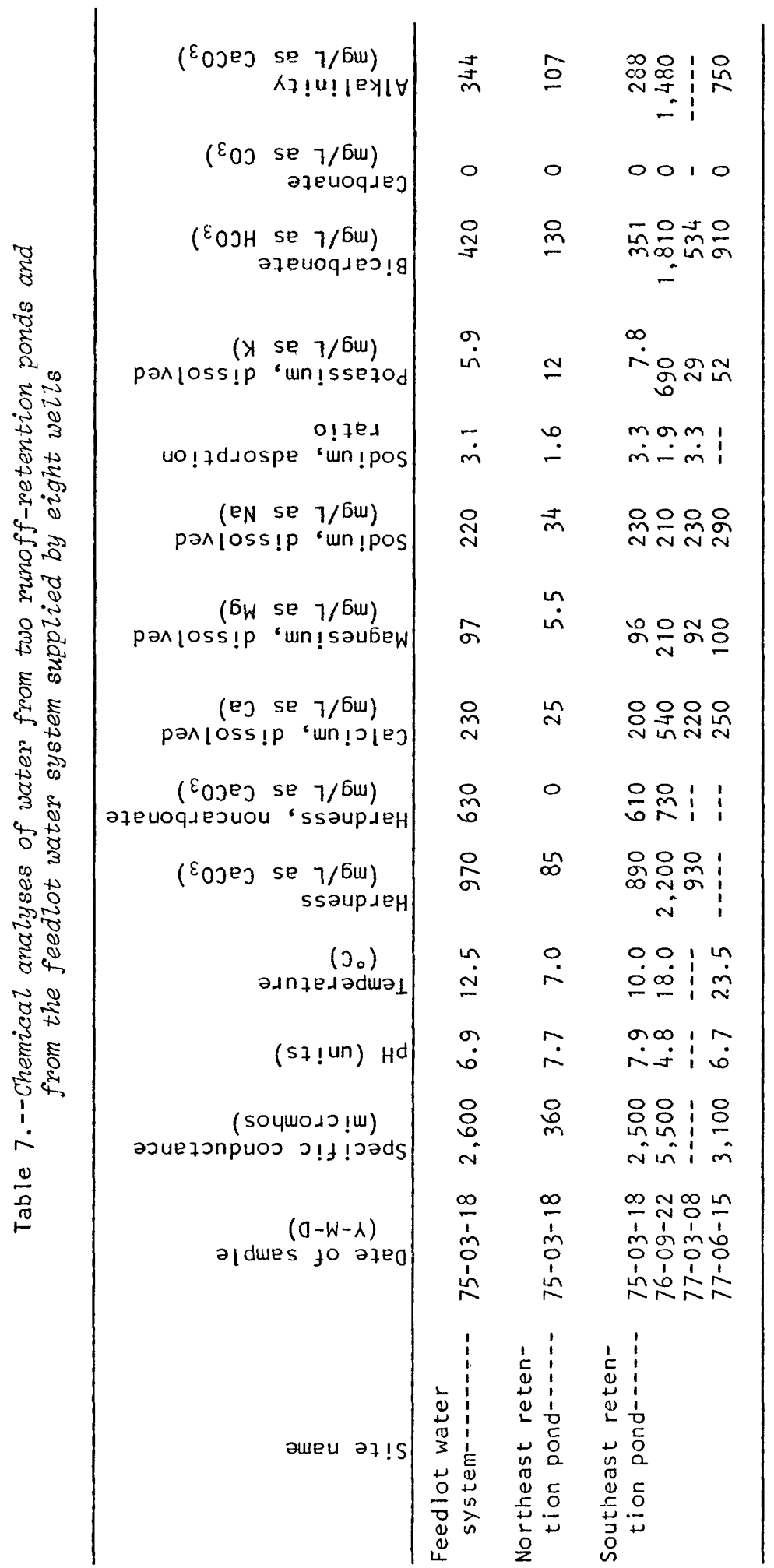




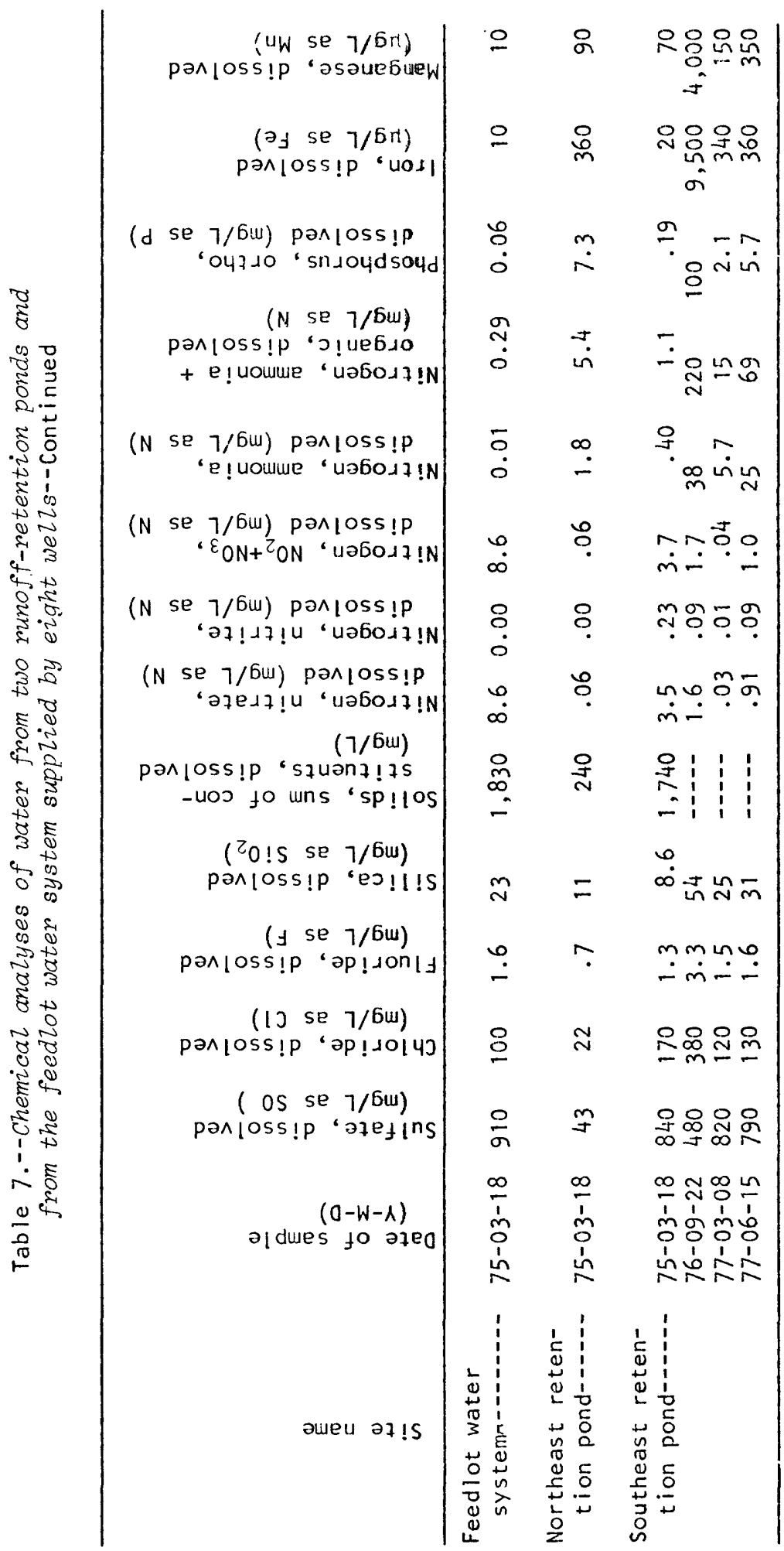


Table 8.--Logs of wells drizled by the U.S. Geological Survey

Thick-

Depth

ness

(feet)

Well 6. Aititude: 4,579 feet

Topsoil, fine-grained-_.-.

Sand, fine-grained; clay

$5 \quad 5$

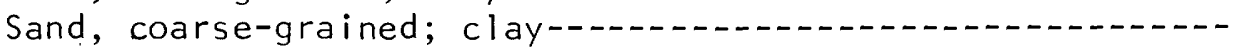

Sand, coarse-grained; fine- to coarse-grained gravel-.-.---

Sand, coarse-grained; some clay; some fine- to coarse-

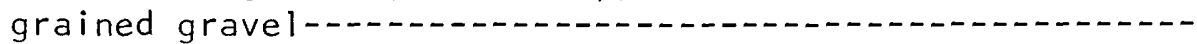

$5 \quad 10$

$5 \quad 15$

$5 \quad 20$

5

25

Gravel, fine- to coarse-grained; some coarse-grained sand;

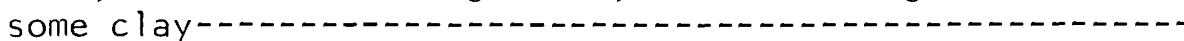

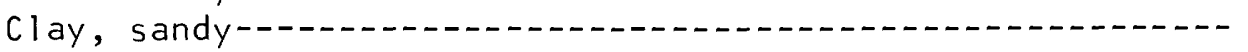

Clay, sandy; some coarse-grained sand; some fine- to

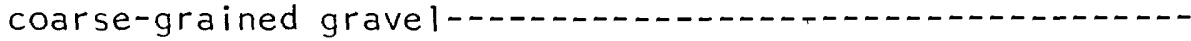

Gravel, fine- to coarse-grained; sandy clay-............

Clay, sandy; fine- to coarse-grained gravel-.............

Sand, coarse-grained; some fine- to medium-grained gravel;

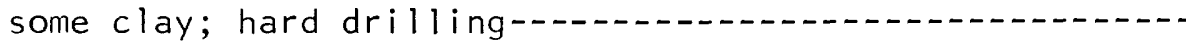

Gravel, fine- to coarse-grained, hard drilling-..........

Sand, coarse-grained; some fine- to coarse-grained gravel--

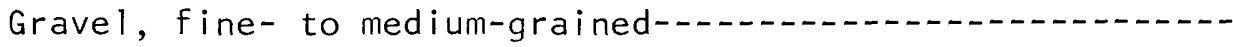

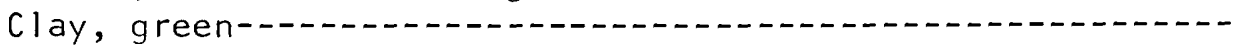

Sand, coarse-grained; fine- to medium-grained gravel; some

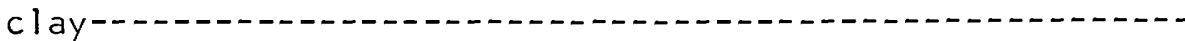

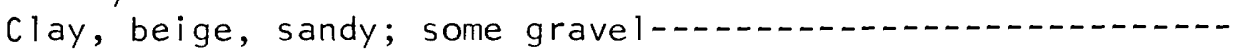

Clay, beige, sandy; some coarse-grained sand; some fine-

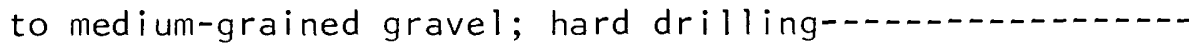

Clay, beige, sandy; some fine-grained gravel-............

Clay, beige, sandy; some dense dark-clay; some fine-grained

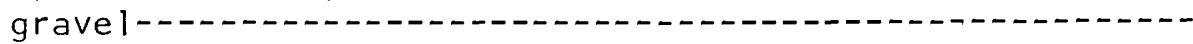

Clay, dense, dark-1a

Shale, dark gray-

$\begin{array}{rl}15 & 40 \\ 5 & 45 \\ 5 & 50 \\ 5 & 55 \\ 5 & 60\end{array}$

$\begin{array}{ll}5 & 65 \\ 5 & 70 \\ 5 & 75 \\ 5 & 80 \\ 1 & 81\end{array}$

$\begin{array}{rr}4 & 85 \\ 5 & 90 \\ 10 & 100 \\ 5 & 105 \\ 5 & 110 \\ 5 & 115 \\ 4 & 119\end{array}$


Table 8.--Logs of wells drilled by the U.S. Geological Survey--Continued

\begin{tabular}{cc}
\hline Thick- & Depth \\
ness & $($ feet $)$ \\
(feet) & \\
\hline
\end{tabular}

Well 11. Altitude: 4,595 feet

Clay, dark; medium-grained gravel; some sand---_------------

Clay, yellow; medium-grained sand; coarse-grained gravel---

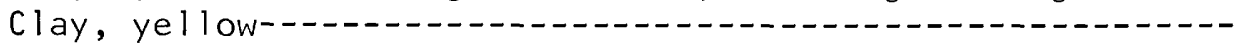

Sand, medium-grained; fine-grained gravel-..............-.

Sand, medium-grained; medium-grained gravel--.-.-.-.-.-----

Sand, coarse-grained; coarse-grained gravel-_..............

Sand, fine; some clay; some fine-grained gravel------------

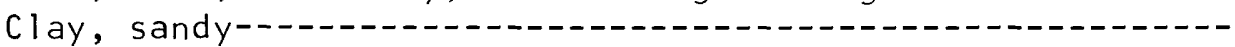

Gravel, medium-grained; some clayey sand; some coarse-

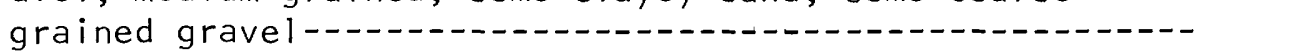

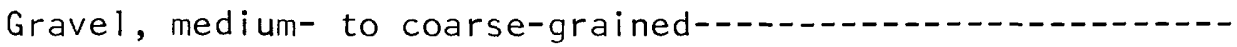

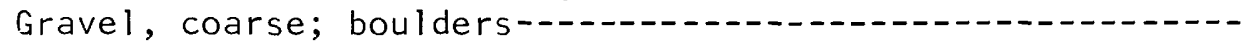

Clay, sandy--

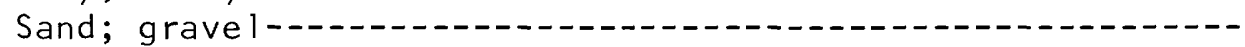

Clay, sandy; some sand; some fine-grained gravel--.--------

Clay, light brown, sandy---1.--

Clay, gray, tight-1

Clay, dark gray, tight-1-.-

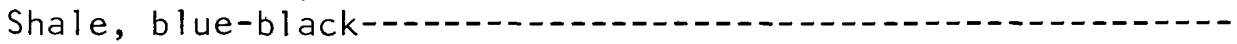

\section{Well 13. Altitude: 4,599 feet}

Sand, fine-grained, clayey-15

Sand, fine- to medium-grained---_-n 50

Sand, clayey-_...-. 323

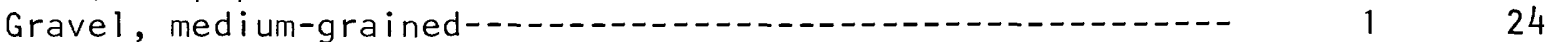

Sand, medium-grained; some fine-grained gravel------------ 428

Sand, fine- to coarse-grained; fine- to medium-grained

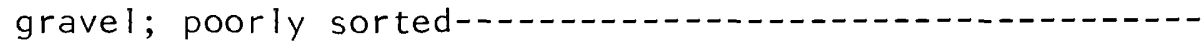

Sand, medium- to coarse-grained; fine- to medium-grained gravel-1...-...--

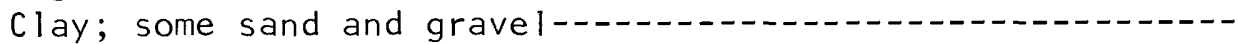

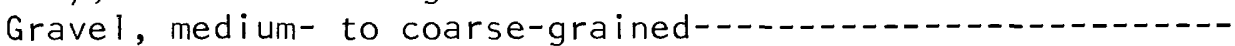

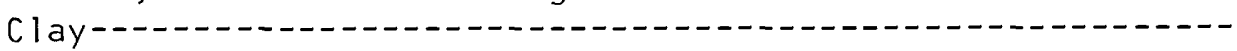

Sand, coarse-grained, fine- to medium-grained gravel------Shale--1-n-

$\begin{array}{rr}7 & 35 \\ 11 & 46 \\ 3 & 49 \\ 31 & 80 \\ 28 & 108 \\ & \\ 20 & 128 \\ 3 & 131\end{array}$


Table 8.--Logs of wells drilled by the U.S. Geological Survey--Continued

Thick- Depth
ness
$($ feet $)$

Well 18. Altitude: 4,589 feet

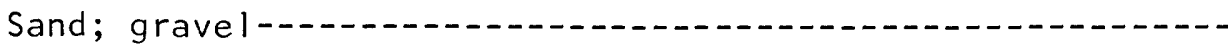

Sand, medium- to coarse-grained; fine- to coarse-grained

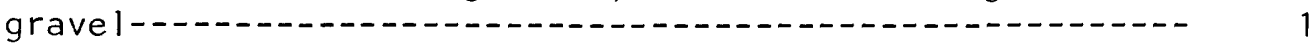

Clay, sandy; brown mica-bearing clay; some fine-grained

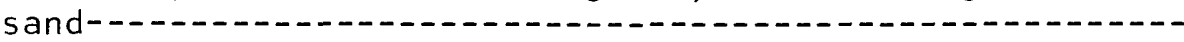

Sand, medium- to coarse-grained; fine- to coarse-grained gravel---1--.--

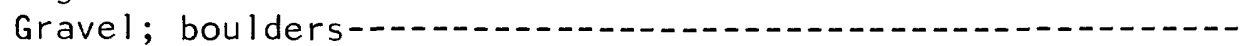

Clay, white-beige, very tight; some boulder chips-........-

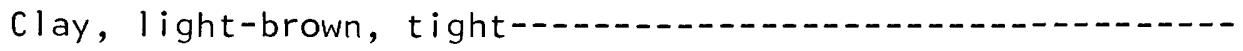

Clay; sand; gravel, poorly sorted; coarse-grained gravel at

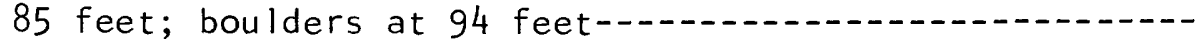

Gravel, medium- to coarse-grained; some broken up boulders;

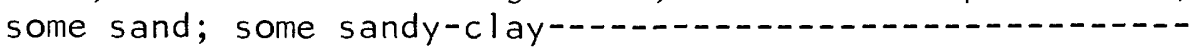

Boulders--..-

Gravel, medium- to coarse-grained; some medium- to coarsegrained sand; some boulders; some yellow-brown clay at

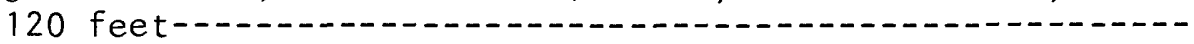

Boulders, unable to drill-a.-

40

Well 23. Altitude: 4,588 feet

Sand, fine- to medium-grained-_.

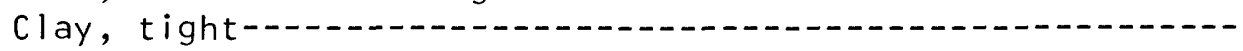

Sand, fine- to medium-grained; some clay-..................

Sand, medium- to coarse-grained; fine-grained gravel-..----

Sand, coarse-grained; fine-grained gravel-...-.

Sand, coarse-grained; fine-grained gravel-...............-

Sand, medium- to coarse-grained; fine-grained gravel--.-.--

Sand, coarse-grained; fine- to medium-grained gravel-...-.-

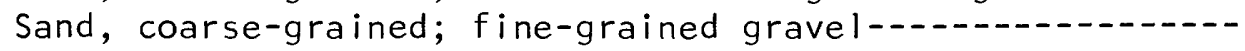

Sand, fine-grained; clay; some fine- to medium-grained

gravel-1..-

Clay, tight; gravel-1

Gravel, fine- to medium-grained; some clay--

Clay-.-.-.-.

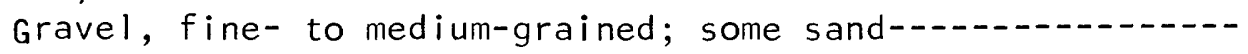

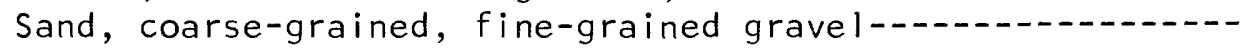


Table 8.--Logs of weIls drizled by the U.S. Geological Survey--Continued

Thickness

(feet)

Well 23. Altitude: 4,588 feet--Continued

Sand, coarse-grained; fine-grained gravel, broken chips---Gravel, fine- to coarse-grained, broken chips-.-......-Gravel, fine- to medium-grained, well-sorted-.............Gravel, medium- to coarse-grained, chips, well-sorted-...-Sand, coarse-grained; medium-grained gravel; some clay--..-

Clay; some medium- to coarse-grained gravel-_............-

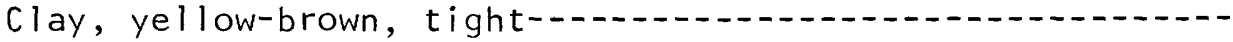

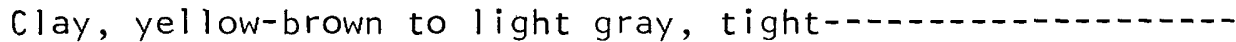
Shale, gray-1...

Well 26. Altitude: 4,584 feet

Sand, clayey; some gravel

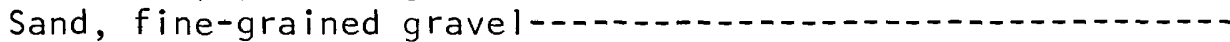

Sand, medium- to coarse-grained; some gravel-..............

Gravel, fine- to medium-grained; some sand-................

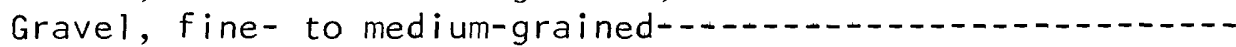

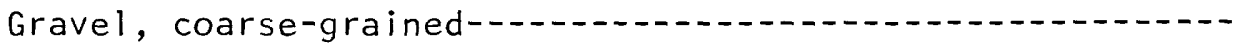
Sand, coarse-grained; fine-grained gravel; some clay-...-.. Shale, gray-
Depth

(feet)

\section{(feet)}


Table 8.--Logs of wells drizled by the U.S. Geological Survey--Continued

\begin{tabular}{ccc}
\hline Thick- & Depth \\
ness & (feet) \\
(feet) & \\
\hline
\end{tabular}

\section{Well 32. Altitude: 4,582 feet}

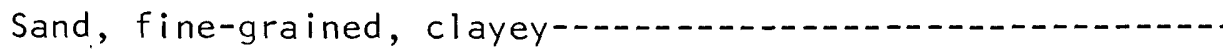

Sand, fine- to medium-grained-1

Sand, silty-...-

Sand, coarse-grained; some fine-grained gravel-........-

Sand, coarse-grained; some fine- to medium-grained gravel-Gravel, fine- to medium-grained-_...-.

Gravel, coarse-grained--....

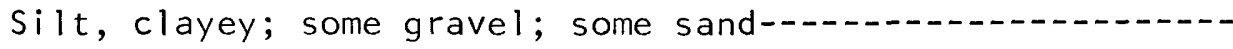

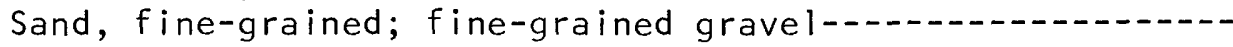

Gravel, medium- to coarse-grained-....-.

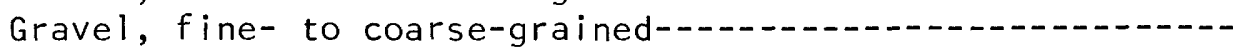

Clay, dark gray-

$\begin{array}{rr}5 & 5 \\ 10 & 15 \\ 5 & 20 \\ 5 & 25 \\ & \\ 5 & 30 \\ 5 & 35 \\ 5 & 40 \\ 5 & 45 \\ 5 & 50 \\ & \\ 10 & 60 \\ 9 & 69 \\ -- & 69\end{array}$

Well 37. Altitude: 4,586 feet

Topsoi 1--

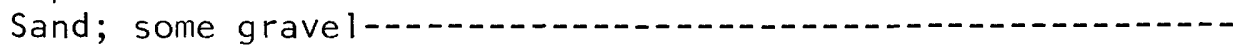

Sand, clayey; sand, fine- to medium-grained; some gray

clay--.--.-.-...-.

Sand, clayey; some fine- to medium-grained gravel-......-.

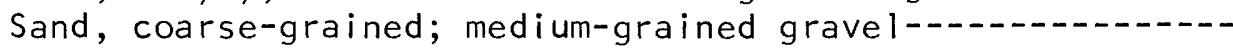

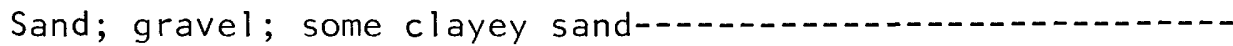

Sand, clayey; fine-grained sand; some gravel-..........-.

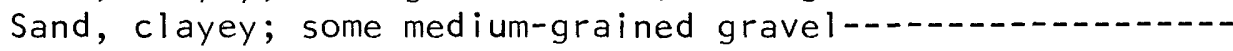

Gravel, medium- to coarse-grained; some sand; some clayey

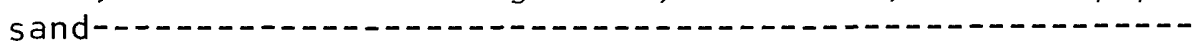

Gravel, coarse-grained; some medium-grained sand-...-.-.--

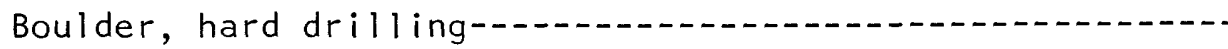

Sand; coarse-grained gravel-1

Shale, black-1

$\begin{array}{rr}5 & 5 \\ 5 & 10 \\ 10 & 20 \\ 5 & 25 \\ 5 & 30 \\ & \\ 10 & 40 \\ 5 & 45 \\ 5 & 50 \\ & \\ 5 & 55 \\ 7 & 62 \\ & \\ 2 & 64 \\ 4 & 68 \\ 1 & 69\end{array}$



Table 9.--Particle-size analyses, statistical total porosity and vertical hydraulic conduc-

\begin{tabular}{|c|c|c|c|c|c|c|c|c|c|c|c|c|}
\hline \multirow[b]{2}{*}{$\begin{array}{l}\text { Depth } \\
\text { of } \\
\text { sample } \\
\text { (feet) }\end{array}$} & \multicolumn{12}{|c|}{$\begin{array}{c}\text { Percentage of sample by particle size by weight } \\
\text { Diameter, in millimeters }\end{array}$} \\
\hline & $\begin{array}{r}\text { ठㅁ } \\
\frac{0}{0} \dot{0}\end{array}$ & 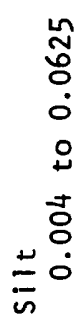 & 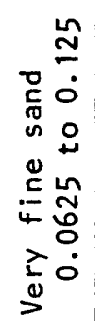 & 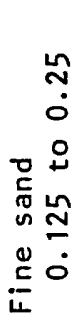 & 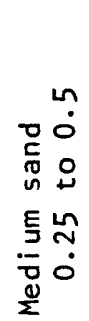 & 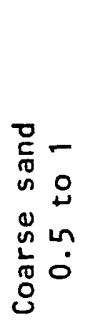 & 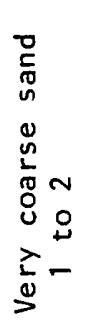 & 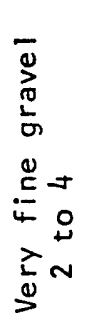 & 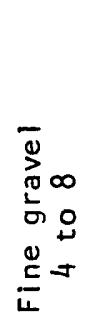 & 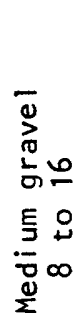 & 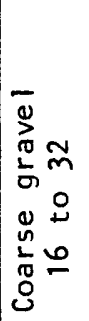 & 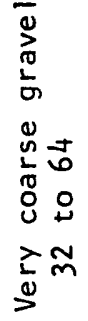 \\
\hline
\end{tabular}

Well 13

\begin{tabular}{|c|c|c|c|c|c|c|c|c|c|c|c|}
\hline $\begin{array}{l}2.0-2.5 \\
3.0-3.5 \\
4.0-4.5 \\
5.0-5.5 \\
7.0-7.5\end{array}$ & $\begin{array}{l}5.4 \\
5.9 \\
5.2 \\
5.2 \\
2.7\end{array}$ & $\begin{array}{r}13.4 \\
14.0 \\
11.0 \\
10.5 \\
6.1\end{array}$ & $\begin{array}{l}10.7 \\
12.2 \\
15.3 \\
14.1 \\
13.1\end{array}$ & $\begin{array}{l}19.9 \\
20.8 \\
33.6 \\
33.1 \\
32.5\end{array}$ & $\begin{array}{l}14.0 \\
18.8 \\
19.3 \\
18.7 \\
22.4\end{array}$ & $\begin{array}{l}20.3 \\
14.1 \\
14.2 \\
14.3 \\
22.0\end{array}$ & $\begin{array}{r}13.2 \\
9.2 \\
1.5 \\
4.1 \\
1.1\end{array}$ & $\begin{array}{l}3.1 \\
2.8 \\
0 \\
0 \\
0\end{array}$ & $\begin{array}{l}0 \\
1.1 \\
0 \\
0 \\
0\end{array}$ & $\begin{array}{l}0 \\
1.0 \\
0 \\
0 \\
0\end{array}$ & 0 \\
\hline $\begin{array}{l}9.0-9.5 \\
1.0-11.5 \\
3.0-13.5 \\
6.0-16.5 \\
8.5-19.0\end{array}$ & $\begin{array}{r}4.7 \\
11.4\end{array}$ & $\begin{array}{r}7.1 \\
28.9\end{array}$ & $\begin{array}{l}10.4 \\
12.4 \\
14.3 \\
19.1 \\
25.6\end{array}$ & $\begin{array}{l}27.2 \\
45.9 \\
34.1 \\
42.7 \\
23.1\end{array}$ & $\begin{array}{r}24.6 \\
33.9 \\
21.2 \\
22.5 \\
7.8\end{array}$ & $\begin{array}{r}30.9 \\
4.3 \\
14.5 \\
3.7 \\
3.1\end{array}$ & $\begin{array}{c}2.5 \\
0 \\
10.7 \\
.2 \\
.2\end{array}$ & $\begin{array}{l}0 \\
0 \\
0 \\
0 \\
0\end{array}$ & $\begin{array}{l}0 \\
0 \\
0 \\
0 \\
0\end{array}$ & $\begin{array}{l}0 \\
0 \\
0 \\
0 \\
0\end{array}$ & $\begin{array}{l}0 \\
0 \\
0 \\
0 \\
0\end{array}$ \\
\hline $\begin{array}{l}21.5-22.0 \\
24.5-25.0 \\
26.0-26.5 \\
28.5-29.0 \\
29.0-29.5\end{array}$ & $\begin{array}{l}4.6 \\
4.6 \\
4.2 \\
1.5 \\
1.6\end{array}$ & $\begin{array}{r}11.3 \\
4.8 \\
9.2 \\
7.2 \\
6.4\end{array}$ & $\begin{array}{r}13.8 \\
19.6 \\
8.9 \\
4.0 \\
3.6\end{array}$ & $\begin{array}{r}22.2 \\
30.6 \\
16.4 \\
11.0 \\
7.9\end{array}$ & $\begin{array}{r}16.2 \\
23.9 \\
9.8 \\
15.4 \\
12.7\end{array}$ & $\begin{array}{l}11.0 \\
12.8 \\
14.6 \\
14.7 \\
14.7\end{array}$ & $\begin{array}{r}14.5 \\
3.7 \\
13.6 \\
15.8 \\
16.7\end{array}$ & $\begin{array}{c}6.2 \\
0 \\
15.6 \\
15.7 \\
21.6\end{array}$ & $\begin{array}{l}.3 \\
0 \\
6.0 \\
11.9 \\
11.1\end{array}$ & $\begin{array}{l}0 \\
0 \\
1.8 \\
2.7 \\
3.8\end{array}$ & $\begin{array}{l}0 \\
0 \\
0 \\
0 \\
0\end{array}$ \\
\hline
\end{tabular}

Well 28

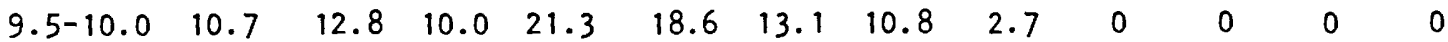


characteristics, specific gravity, moisture content, tivity for samples from observation wells 13 and 28

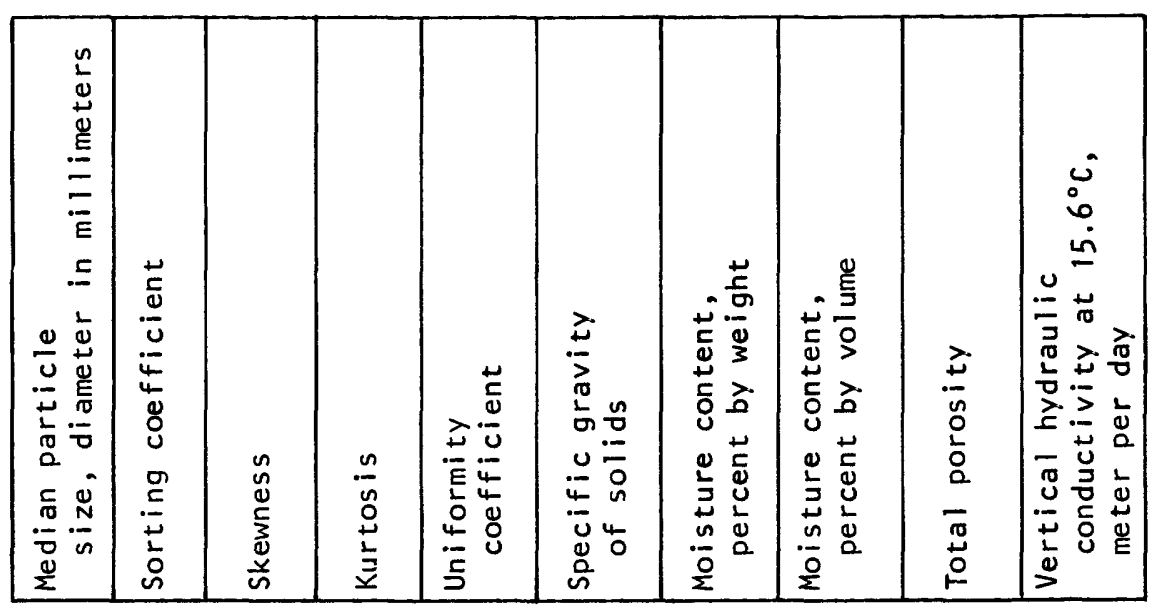

Well 13

\begin{tabular}{|c|c|c|c|c|c|c|c|c|c|}
\hline $\begin{array}{r}0.26 \\
.23 \\
.18 \\
.19 \\
.23\end{array}$ & $\begin{array}{l}2.8 \\
2.7 \\
2.0 \\
2.0 \\
1.9\end{array}$ & $\begin{array}{l}1.0 \\
.95 \\
.99 \\
1.1 \\
1.2\end{array}$ & $\begin{array}{r}0.24 \\
.19 \\
.21 \\
.20 \\
.25\end{array}$ & $\begin{array}{r}32 \\
21 \\
7.7 \\
8.5 \\
4.5\end{array}$ & $\begin{array}{l}2.67 \\
2.68 \\
2.69 \\
2.69 \\
2.69\end{array}$ & $\begin{array}{l}4.76 \\
6.94 \\
5.68 \\
5.36 \\
4.40\end{array}$ & $\begin{array}{r}8.38 \\
11.38 \\
8.52 \\
9.38 \\
6.38\end{array}$ & $\begin{array}{l}34.23 \\
38.65 \\
44.11 \\
34.90 \\
46.93\end{array}$ & \begin{tabular}{l}
2.2 \\
\hdashline .86
\end{tabular} \\
\hline $\begin{array}{l}.31 \\
.21 \\
.23 \\
.17 \\
.081\end{array}$ & $\begin{array}{l}1.9 \\
1.5 \\
1.9 \\
1.6 \\
2.3\end{array}$ & $\begin{array}{l}1.0 \\
1.1 \\
1.3 \\
.91 \\
.76\end{array}$ & $\begin{array}{l}.29 \\
.26 \\
.19 \\
.22 \\
.25\end{array}$ & $\begin{array}{c}4.6 \\
2.7 \\
3.9 \\
4.2 \\
37\end{array}$ & $\begin{array}{l}---- \\
--- \\
-- \\
2.70 \\
2.70\end{array}$ & $\begin{array}{r}3.80 \\
5.06 \\
3.95 \\
3.22 \\
10.52\end{array}$ & $\begin{array}{r}5.7 \\
5.85 \\
4.44 \\
14.73\end{array}$ & $\begin{array}{l}44.03 \\
-14.98 \\
48.04 \\
47.76\end{array}$ & 5.2 \\
\hline $\begin{array}{l}.24 \\
.20 \\
.54 \\
.83 \\
1.1\end{array}$ & $\begin{array}{l}2.8 \\
1.9 \\
3.6 \\
3.1 \\
2.9\end{array}$ & $\begin{array}{l}1.4 \\
1.0 \\
.89 \\
.96 \\
.75\end{array}$ & $\begin{array}{l}.20 \\
.22 \\
.24 \\
.22 \\
.24\end{array}$ & $\begin{array}{l}12 \\
4.0 \\
25 \\
17 \\
19\end{array}$ & $\begin{array}{l}2.71 \\
2.69 \\
2.69 \\
2.69 \\
2.69\end{array}$ & $\begin{array}{l}2.47 \\
4.34 \\
2.35 \\
2.71 \\
1.36\end{array}$ & $\begin{array}{r}6.55 \\
4.63 \\
-\end{array}$ & $\begin{array}{l}-1 . \\
43.90 \\
36.42\end{array}$ & $\begin{array}{r}.93 \\
-.9 \\
.04 \\
.04\end{array}$ \\
\hline \multicolumn{10}{|c|}{ Well 28} \\
\hline
\end{tabular}

$\begin{array}{llllllllll}.21 & 2.8 & .83 & .19 & 216 & 2.67 & 9.66 & 15.55 & 39.70 & 3.4\end{array}$ 
Table 10.--Monthly precipitation, in inches, from January 1975 to September 1978 at weather stations operated by the feedlot owner at the feedlot and by the U.S. Department of Commerce, National Weather Service at Greeley, Colo.

\begin{tabular}{|c|c|c|c|c|c|c|c|c|}
\hline \multirow{2}{*}{ Month } & \multicolumn{2}{|c|}{1975} & \multicolumn{2}{|c|}{1976} & \multicolumn{2}{|c|}{1977} & \multicolumn{2}{|c|}{1978} \\
\hline & Feedlot & Greeley & Feedlot & Greeley & Feedlot & Greeley & Feedlot & Greeley \\
\hline Jan. & 0.12 & 0.02 & 0.02 & 0.10 & 0.38 & 0.13 & 0.40 & 0.50 \\
\hline Feb. & .12 & .14 & .14 & .50 & .00 & trace & .10 & .13 \\
\hline Mar. & .58 & .75 & .28 & .49 & .30 & .44 & .15 & .09 \\
\hline Apr. & 1.26 & 1.86 & 1.13 & 1.76 & 2.87 & 2.21 & 2.48 & 1.83 \\
\hline May & 4.88 & 5.34 & 3.13 & 3.69 & 1.20 & 1.91 & 3.40 & 5.22 \\
\hline June & .80 & 2.02 & 1.42 & .74 & .38 & .70 & 1.70 & 1.37 \\
\hline July & 3.00 & 2.72 & 1.90 & 1.36 & .70 & 2.16 & .93 & .70 \\
\hline Aug. & 1.84 & .63 & .85 & .90 & 1.02 & 1.48 & .64 & .53 \\
\hline Sept. & .64 & .41 & 2.21 & 1.92 & .20 & .14 & .00 & trace \\
\hline oct. & .25 & .32 & .40 & .35 & .07 & .10 & --- & -- \\
\hline Nov. & .65 & 1.00 & .04 & .08 & .45 & .42 & --- & --- \\
\hline Dec. & .42 & .87 & .05 & .12 & .72 & .29 & --- & --. \\
\hline Total & 14.56 & 16.08 & 11.57 & 12.01 & 8.29 & 9.98 & 9.80 & 10.37 \\
\hline
\end{tabular}


